\title{
Performance Impact of Improved Departure Time Prediction Relative to Sector Demand \& Arrival Time Predictability
}

Frederik Könnemann B.Eng. 



\title{
Performance Impact of Improved Departure Time Prediction Relative to Sector Demand \& Arrival Time Predictability
}

\author{
Master of Science Thesis \\ For obtaining the degree of Master of Science in Aerospace \\ Engineering at Delft University of Technology
}

Frederik Könnemann B.Eng.

$12-12-2014$

Faculty of Aerospace Engineering - Delft University of Technology 


\section{T⿱㇒冋UDelft}

Copyright (c) Frederik Könnemann B.Eng. All rights reserved. 


\section{Delft University Of Technology \\ DEPARTMENT OF}

Air Transport \& Aerospace Operations

The undersigned hereby certify that they have read and recommend to the Faculty of Aerospace Engineering for acceptance a thesis entitled "Performance Impact of Improved Departure Time Prediction Relative to Sector Demand \& Arrival Time Predictability" by Frederik Könnemann B.Eng. in partial fulfillment of the requirements for the degree of Master of Science.

Dated: $\underline{12-12-2014}$

Prof.dr.ir. Richard Curran

Ir. Sönke Mahlich

Dr. Wenjing Zhao

Dr.ir. Clark Borst 



\section{Abstract}

This thesis deals with traffic forecasts of Airspace Users for Air Navigation Service Providers. Currently there is a high amount of uncertainty in the traffic forecast. Air Traffic Flow Managers anticipate for unforeseen traffic by increasing the forecasted maximum capacity threshold by more than 10\%. The European ATM research program SESAR aims ultimately to reduce the difference between the forecast and real maximum capacity threshold by less than $3 \%$. Prediction uncertainty results in sector over-load and sub-optimal traffic flow in the air transportation system. The objective of this thesis is to investigate sector demand predictability by quantification of the difference between real and forecast traffic, and evaluate the predictability improvement by improvement of departure time predictions.

Statistical analysis is performed by plotting time \& count uncertainty against look-ahead time to sector entry. On a busy day, for a look-ahead time of 2 hours and longer, flights have a higher probability to be delayed than to be earlier as planned. A comparison is made between the forecast and real number of flights entering a sector for a given time of day window. Looking at the forecast time period, it can be seen that some forecasted flights did not enter in the window anymore (out), and some additional flights entered in the window that were initially not forecasted (in). 'In' and 'out' flights can be explained due to flights being earlier or delayed, or flights deviating from the planned route. Looking at 10 minutes before entry, about 30\% to $40 \%$ are in/out flights, which is a large amount. In general, for a look-ahead of 0 to 3 hours, there are more 'out' than 'in' flights resulting in an over-prediction. Over-prediction means that there are more flights anticipated than really entered for a given time window.

In order to reduce over-prediction, it is suggested, taking safety into account, to reduce the number of 'out' flights that deviate from the planned route. For a high capacity Maastricht Upper Area Control sector on a normal day, a $5 \%$ decrease of these 'out' flights, reduces the over-prediction by $10 \%$. Furthermore, flight phases that are major causes of uncertainty are descent, taxi and the slot allocation process.

A departure time prediction improvement of $50 \%$ results in $20 \%$ arrival time error reduction, and 30\% mean sector entry time error reduction, for a 6 hour look-ahead time. The used sensitivity method does not yield realistic sector occupancy count because the effect of changed ATC procedures due to improved predictability is not incorporated. 



\section{Acknowledgements}

I wish to thank my supervisors Sönke Mahlich \& Richard Curran for their guidance in this research project and the opportunities they gave me. I would also like to thank Richard Irvine, Andrew Hately, Andre Marayat, Brice Genestier, and Francoise Guenin-Rambaud for their unselfish advice, involvement and support.

I do not only want to thank my parents and sister for the variety of support they gave me during this research, but also for all the support I received during my student life.

Finally I would like to thank Menno Galama, Anastasiia Khomenok, Steven Kirby, Yorick Dantuma, Akke Toeter, Max Stracke, Bart Wolfs, and Jóse Ripperda who where there for me in good and bad times.

Delft, The Netherlands

Frederik Könnemann B.Eng.

12-12-2014 



\section{Contents}

$\begin{array}{ll}\text { Abstract } & \text { v }\end{array}$

$\begin{array}{ll}\text { Acknowledgements } & \text { vi }\end{array}$

$\begin{array}{ll}\text { List of Figures } & \text { xvi }\end{array}$

List of Tables $\quad$ xviii

1 Introduction $\quad 1$

1.1 Prediction Uncertainty . . . . . . . . . . . . . . . . . 2

1.2 Benefits of Improved Predictability . . . . . . . . . . . . . . . . . 3

1.3 Research Objectives ......................... 4

1.4 Individual Flight Example . . . . . . . . . . . . . . . . . . . . . 4

2 Literature Review $\quad 7$

2.1 Factors Affecting Sector Demand Predictability . . . . . . . . . . . 7

2.1.1 Flight Plan Filing Time . . . . . . . . . . . . . 8

2.1.2 Regulations ..................... . . 9

2.1.3 Predeparture Components . . . . . . . . . . . . . 11

2.1.4 Sector Traffic Type . . . . . . . . . . . . . . . . 11

2.1 .5 Flight Type ..................... 13

2.1 .6 Weather ..................... 14

2.1.7 Operational Impact . . . . . . . . . . . . . . . . 14

2.2 Conclusions ....................... . . 15

3 Modelling Framework $\quad 17$

3.1 Trajectory Predictions . . . . . . . . . . . . . . . . . 17

3.2 Explanation of the Data Extraction Code . . . . . . . . . . . . . . . 19

3.3 Methodology ...................... 20

3.4 Confidence Bounds . . . . . . . . . . . . . . . . . . . . . 22 
4 Departure Time Predictability $\quad 25$

4.1 Population Sample . . . . . . . . . . . . . . . . . . . 25

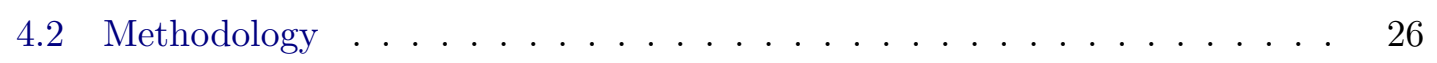

4.3 Confidence Bounds . . . . . . . . . . . . . . . . . . . . . 27

4.4 Individual Flight Example . . . . . . . . . . . . . . . . . . . . . . . . . . . . . . . .

4.5 Departure Time Prediction Error . . . . . . . . . . . . . . . . 28

4.6 Standard Deviation Prediction Error per Airport . . . . . . . . . . . . . 30

4.7 Conclusions . . . . . . . . . . . . . . . . . . . . . 30

5 Sector Demand Predictability $\quad 33$

5.1 Population Sample . . . . . . . . . . . . . . . . . . . 33

5.2 Sector Entry Time Predictability . . . . . . . . . . . . . . . . 35

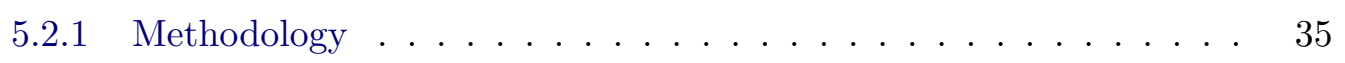

5.2 .2 Individual Flight Example . . . . . . . . . . . . . . . . . . . . . . . . . . . . . .

5.2 .3 Individual Departure Airport . . . . . . . . . . . . . . . . . . . . . . . . . . . . . . . . . .

5.2 .4 Individual Sector . . . . . . . . . . . . . . . . . . 38

5.2 .5 Multiple Sectors . . . . . . . . . . . . . . . . . . . . . . . . . . . 38

5.2 .6 Accumulated Flight Plans . . . . . . . . . . . . . . . . . . 39

5.3 Sector Occupancy Count Predictability . . . . . . . . . . . . . . . . . 41

5.3 .1 Definition . . . . . . . . . . . . . . . . . 41

5.3 .2 Confidence Bounds . . . . . . . . . . . . . . . . . . . . . . . . . 41

$5.3 .3 \quad$ Individual Flight Example . . . . . . . . . . . . . . . . 43

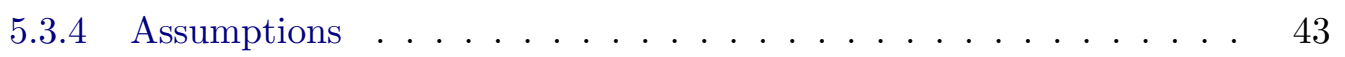

5.3.5 Predicted Sector Occupancy Count . . . . . . . . . . . . . . . 44

5.3.6 Mean \& Standard Deviation Prediction Error . . . . . . . . . . . 45

5.3 .7 Maximum Count Threshold . . . . . . . . . . . . . . . . 48

5.3 .8 Flight Status at Prediction . . . . . . . . . . . . . . . 49

5.3 .9 Inflow \& Outflow Flights . . . . . . . . . . . . . . 50

5.3 .10 Reasons Inflow \& Outflow Flights . . . . . . . . . . . . . . . . 52

5.4 Sector Entry Count Predictability _ . . . . . . . . . . . 56

5.4 Definition . . . . . . . . . . . . . . . 56

5.4 .2 Mean \& Standard Deviation Prediction Error . . . . . . . . . . 58

$5.4 .3 \quad$ Flight Status at Prediction . . . . . . . . . . . . . . 59

5.4 .4 Inflow \& Outflow Flights . . . . . . . . . . . . . . 59

5.5 Conclusions . . . . . . . . . . . . . . . . . . . 61

6 Validation of Actual Traffic Count $\quad 63$

6.1 Sector Occupancy Count . . . . . . . . . . . . . . . . . . . 63

6.2 Sector Entry Count . . . . . . . . . . . . . . . . . . . . 66

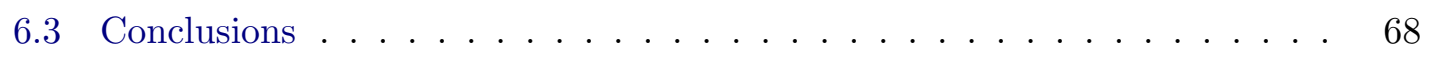


7 Arrival Time Predictability $\quad 69$

7.1 Population Sample . . . . . . . . . . . . . . . . . . . . . . . 69

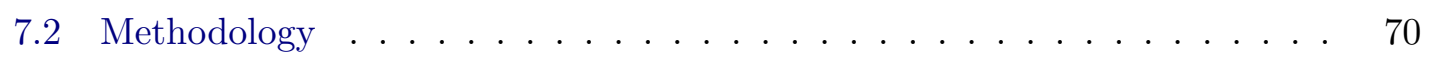

7.3 Confidence bounds . . . . . . . . . . . . . . . . . . . . . . . 71

7.4 Mean Arrival Time Prediction Error . . . . . . . . . . . . . . . . . . 71

7.5 Standard Deviation Arrival Time Prediction Error . . . . . . . . . . . . 72

7.6 Accumulated Flight Plans . . . . . . . . . . . . . . . . . . . . 74

7.7 Conclusions . . . . . . . . . . . . . . . . . . . . . 74

8 Sensitivity Analysis $\quad \mathbf{7 5}$

8.1 Methodology . . . . . . . . . . . . . . . . . . . 75

8.2 Individual Flight Example . . . . . . . . . . . . . . . . . 76

8.3 Sector Demand Predictability . . . . . . . . . . . . . . . . 78

8.3.1 Sector Entry Time Predictability . . . . . . . . . . . . 78

8.3.2 Sector Occupancy Count Predictability . . . . . . . . . . . . 79

8.4 Arrival Time Predictability . . . . . . . . . . . . . . . . . . . . 82

8.5 Conclusions . . . . . . . . . . . . . . . . . . . 83

9 Performance Impact \& Recommendations $\quad 85$

9.1 Performance Impact $\ldots \ldots \ldots \ldots$

9.2 Recommendations . . . . . . . . . . . . . . 88

10 Conclusions $\quad 89$

10.1 Conclusions . . . . . . . . . . . . . . . . . . . . 89

10.2 Limitations \& Future Work . . . . . . . . . . . . . . . . 90

$\begin{array}{ll}\text { References } & 93\end{array}$

A Flight Prediction Update Message $\quad 95$

B Departure Time Predictability for Normal \& Busy Days 97

C Sector Occupancy Count Flight Status at Prediction 101

D Sector Occupancy Count Inflow \& Outflow Flights 105

E Sector Entry Count Flight Status at Prediction $\quad 109$

F Sector Entry Count Inflow \& Outflow Flights 111 


\section{List of Figures}

1.1 Forecast ATC workload versus real ATC workload (estimation) [6] . . . . 3

1.2 Trajectory prediction uncertainty over time horizon (estimation) [6]. . . . 4

1.3 Geographic top-down view of predicted \& actual trajectory for example

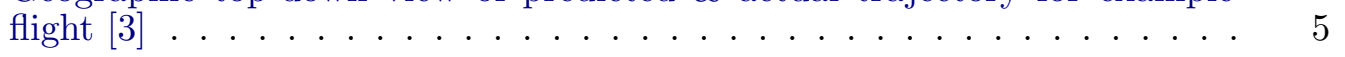

1.4 Example of look-ahead time definition, for individual flight. . . . . . . . 6

2.1 Absolute sector entry time prediction error. . . . . . . . . . . . . . . . 10

2.2 Sector entry time prediction error for flights with and without regulation . 10

2.3 Predeparture component of sector entry time prediction error for good weather ............................... 11

2.4 Sector occupancy count prediction error for active vs. proposed flights at different times of the day. $[10] \ldots \ldots 12$

2.5 Mean and standard deviation of peak count prediction error. . . . . . . . 13

2.6 Peak count cumulative distribution function. . . . . . . . . . . . . . . 13

2.7 Sector entry time prediction error for different flight types \& weather types. 14

3.1 Main data flows of ETFMS [1] . . . . . . . . . . . . . . . . 17

3.2 Sector count prediction example from the Flow Manager's perspective [3]. 18

3.3 Flow diagram to obtain sector occupancy count in section 5.3, from EFD file. . . . . . . . . . . . . . . . . . . . 20

3.4 Additional part of flow diagram in figure 3.3 to obtain altered sector occupancy count in section 8.3.2. . . . . . . . . . . . . . . . 20

3.5 Example positive and negative prediction error. . . . . . . . . . . . . 22

4.1 Geographical top-down view of the airspaces included in the analysis. . . 26

4.2 Example plot of kernel density estimate and histogram [2] . . . . . . . . 27

4.3 Example prediction error for 1 hour look-ahead, for individual flight. . . . 27

4.4 Take-off time prediction error during taxi, and at off-block time, for regulated flights . . . . . . . . . . . . . . . . . . . . . 29 
4.5 Sector entry time prediction error at initial flight plan, at off-block time, and at take-off, for regulated flights . . . . . . . . . . . . .

4.6 Take-off time prediction error at different time instances for non-regulated flights. . . . . . . . . . . . . . . . . .

4.7 Take-off time prediction error at last slot issue time for regulated flights .

4.8 Take-off time, and sector entry time prediction error at initial flight plan, non-regulated flights. . . . . . . . . . . . . . . .

4.9 Take-off time, and sector entry time prediction error at initial flight plan,

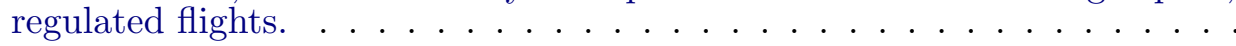

4.10 Standard deviation of the departure time prediction error per airport. Error for regulated flights at last slot issue time, error for non-regulated flights at 30 minutes before take-off. . . . . . . . . . . . . . . . . . .

5.1 Geographical top-down view of analyzed sectors [3]. . . . . . . . . . . . 34

5.2 Example normal distribution plot. [2] . . . . . . . . . . . . . . 36

5.3 Prediction error outliers, for sector Koksy High. . . . . . . . . . . . . . 36

5.4 Example of curve fit through prediction error vs. look-ahead, for individual flight. . . . . . . . . . . . . . . . . .

5.5 Sector entry time prediction error, last available predictions before entry. Flights from London Heathrow. Sector Koksy High. . . . . . . . . . . . .

5.6 Sector entry time prediction error, for flights from different origin airports. Sector Koksy High. . . . . . . . . . . . . . . . . . . . .

5.7 Sector entry time prediction error, for all flights on 28th of June 2013. Sector Koksy High. . . . . . . . . . . . . . . . . . . . . .

5.8 Mean sector entry time prediction error for all flights on 28th of June 2013. Sector Koksy High . . . . . . . . . . . . . . . . . . . . . . . 39

5.9 Mean sector entry time prediction error for multiple sectors . . . . . . . 39

5.10 Standard deviation sector entry time predication error for multiple sectors. 40

5.11 Accumulated flight plans for multiple sectors. . . . . . . . . . . . . . 40

5.12 Example occupancy count. Skip time $=10$ minutes and window $=10$ minutes. . . . . . . . . . . . . . . . . . .

5.13 Example of forecast and real occupancy count. . . . . . . . . . . . . 43

5.14 Sector occupancy count prediction for time of day window 12:00 to 12:10. Sector Koksy High . . . . . . . . . . . . . . . . . .

5.15 Sector occupancy count prediction for several time of day windows. Sector: Koksy High. . . . . . . . . . . . . . . . . . . . . . . 45

5.16 Example sector occupancy count prediction error for 1 hour look-ahead. . 46

5.17 Mean sector occupancy count prediction error. Sector Koksy High . . . 46

5.18 Mean \& standard deviation sector occupancy count prediction error. Dot is mean, $1 / 2$ bar length is 1 standard deviation. Sector Koksy High. . . .

5.19 Sector occupancy count prediction error in aircraft, for multiple sectors. Day is 28 th of June 2013 unless stated otherwise. . . . . . . . . . . . .

5.20 Sector occupancy count prediction error in percentage points, for multiple sectors. Day is 28th of June 2013 unless stated otherwise. . . . . . . . . .

5.21 Predicted and actual sector occupancy count for 1 hour look-ahead. Sector Koksy High. . . . . . . . . . . . . . . . . . 
5.22 Example snapshot of predicted traffic at 1 hour look-ahead. . . . . . . . .

5.23 Example stacked bar flight status at prediction, based on traffic in figure

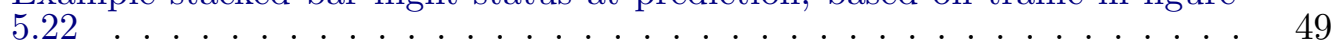

5.24 Sector occupancy count flight status at prediction. Sector Koksy High . .

5.25 Example outflow, stable, and inflow scenarios for individual flight. Lookahead is 1 hour . . . . . . . . . . . . . . . .

5.26 Sector occupancy count inflow, outflow, and stable flights for time of day window 11:50 - 12:00. Look-ahead is 1 hour. Sector Koksy High. . . . . .

5.27 Sector occupancy count inflow, outflow, and stable flights between 08:00 and 20:00, for different look-ahead times. Sector Koksy High. . . . . . . .

5.28 Sector occupancy count inflow, outflow, and stable flights for 1 hour lookahead. Sector Koksy Low on 5 th of March 2014 . . . . . . . . . . . . . .

5.29 Mean sector occupancy count relative over-prediction, extracted from figure

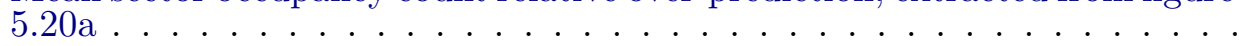

5.30 Example outflow flight due to route deviation. . . . . . . . . . . . . . 54

5.31 Example inflow flight due to late flight plan filing for individual flight. . . 54

5.32 Example entry count. Skip time $=20$ minutes and window $=60$ minutes. $\quad 56$

5.33 Sector entry count predictions for 06:00 to 07:00. Sector Koksy High. . . . 56

5.34 Sector entry count prediction error in aircraft, for multiple sectors. Day is 28th of June 2013 unless stated otherwise . . . . . . . . . . . . . .

5.35 Sector entry count prediction error in percentage points, for multiple sectors. Day is 28th of June 2013 unless stated otherwise . . . . . . . . . . .

5.36 Sector entry count flight status at prediction for different look-ahead times. Sector Koksy High . . . . . . . . . . . . . . . . .

5.37 Sector entry count flow diagram for time of day window 06:00 to 07:00, for different look-ahead times. Sector: Koksy High. . . . . . . . . . . . .

5.38 Sector entry count inflow, outflow, and stable flights between 08:00 and 20:00, for different look-ahead times. Sector Koksy High. . . . . . . . . . .

7.1 Example of arrival time prediction error for the 10 minute slice after take-off. 71

7.2 Mean arrival time prediction error for flights with approximately 1,2, and 3 hour flight time. . . . . . . . . . . . . . .

7.3 Standard deviation of arrival time prediction error for flights with approximately 1,2 , and 3 hours flight time. . . . . . . . . . .

7.4 Standard deviation of prediction error for flights with approximately 1 hour flight time, with quantification of error reductions at several time instances. 73

7.5 Standard deviation of arrival time prediction error for flights with approximately 1 hour flight time for different traffic levels. . . . . . . . . . . .

7.6 Percentage of accumulated flight plans before take-off, for flights with 1 hour flight time.. . . . . . . . . . . . . . .

8.1 Example prediction error for the unaltered and altered individual flight predictions based on table 8.2.

8.2 Mean sector entry time prediction error sensitivity for 3 sector, departure time prediction error reduction done at 1 hour before off block. . . . . . .

8.3 Mean sector entry time absolute prediction error sensitivity, departure time prediction error reduction done at different times before off block. Sector Koksy High. . . . . . . . . . . . . . . . . . . . . . . 
8.4 Mean sector occupancy count prediction error sensitivity for departure time prediction error reductions at different times before off block. Sector Koksy High. . . . . . . . . . . . . . . . . . . . .

8.5 Sector occupancy count prediction error sensitivity for 3 sectors. Departure time error reduction done at 1 hour before off block. . . . . . . . . . . .

8.6 Standard deviation arrival time prediction error sensitivity for flight with 1 hour flight time. Departure time error reduction done at 1 hour before off block. . . . . . . . . . . . . . . . . . .

A.1 Prediction update message for individual flight. . . . . . . . . . . .

B.1 Take-off time, and sector entry time prediction error at initial flight plan, non-regulated flights. . . . . . . . . . . . . . . .

B.2 Take-off time, and sector entry time prediction error at initial flight plan, regulated flights. . . . . . . . . . . . .

B.3 Take-off time prediction error during taxi, and at off-block time, for regulated flights . . . . . . . . . . . . . .

B.4 Sector entry time prediction error at initial flight plan, at off-block time, and at take-off, for regulated flights . . . . . . . . . . . .

B.5 Take-off time prediction error at different time instances for non-regulated flights. . . . . . . . . . . . . . . . . . . . .

B.6 Take-off time prediction error at last slot issue time for regulated flights .

B.7 Standard deviation of the departure time prediction error per airport. Error for regulated flights at last slot issue time, error for non-regulated flights at 30 minutes before take-off. . . . . . . . . . . . . . . . . . . .

C.1 Sector occupancy count flight status at prediction for multiple sectors. Look-ahead is 10 minutes. . . . . . . . . . . . . . . .

C.2 Sector occupancy count flight status at prediction for multiple sectors. Look-ahead is 60 minutes.

C.3 Sector occupancy count flight status at prediction for multiple sectors.

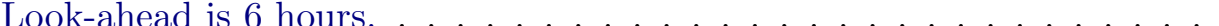

D.1 Sector occupancy count inflow \& outflow for multiple sectors. Look-ahead is 10 minutes. . . . . . . . . . . . . . . . . .

D.2 Sector occupancy count inflow \& outflow flights for multiple sectors. Lookahead is 60 minutes. . . . . . . . . . . . . . . . .

D.3 Sector occupancy count inflow \& outflow flights for multiple sectors. Lookahead time is 6 hours. . . . . . . . . . . . . . . . . . . .

E.1 Sector entry count flight status at prediction for multiple sectors. Lookahead is 60 minutes.

F.1 Sector entry count inflow \& outflow flights for multiple sectors. Look-ahead

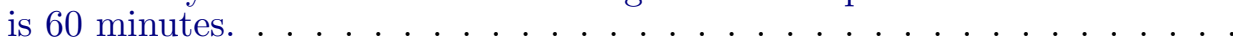




\section{List of Tables}

1.1 Prediction for individual example flight. . . . . . . . . . . . . . 5

2.1 Sample size and analyzed metrics for relevant articles. . . . . . . . . . 7

2.2 Factors affecting sector demand predictions. . . . . . . . . . . . 8

2.3 Mean and standard deviation for sector entry time prediction error [11] . 9

2.4 Absolute mean and standard deviation for departure time prediction error

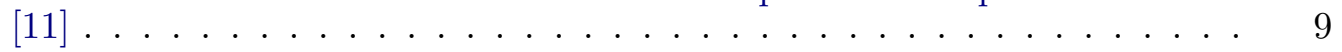

2.5 Mean and standard deviation for 'time over' prediction error . . . . . . 11

5.1 Characteristics of sectors that are analyzed. . . . . . . . . . . . 33

5.2 Goodness of fit values for prediction error vs. look-ahead curve fit. Sector Koksy High . . . . . . . . . . . . . . . . . . . . . . . 35

5.3 Goodness of fit values for prediction error vs. look-ahead curve fit. Sector Lux Low . . . . . . . . . . . . . . . . . . 35

5.4 Flight list of actual \& predicted count for window 09:20 - 09:30. . . . . . . 44

5.5 Reasons for inflow, outflow, and stable flights for multiple sectors for 3 different look-ahead times. . . . . . . . . . . . . . . . 55

5.6 List of actual and predicted flights for window 06:00 to 07:00 . . . . . . 57

6.1 Actual entry and exit times of flight AA94129641 in NEST and EFD. . . 63

6.2 Additional flights for NEST data compared with thesis algorithm (table 6.3) 64

6.3 Actual sector occupancy count comparison between NEST and thesis algorithm. Sector Jever Low. . . . . . . . . . . . . . . . . . . 65

6.4 Actual sector entry count comparison between NEST and thesis algorithm. Sector Koksy High . . . . . . . . . . . . . . . . . . . 67

7.1 Number of daily flights with flight time 1, 2, and 3 hours. . . . . . . . 70

8.1 Trade-off between historical data alteration \& fast time simulation. . . . . 76 
8.2 Example of altered \& unaltered trajectory predictions for the British Airways flight. . . . . . . . . . . . . . . .

8.3 Inflow \& outflow flights, for departure time error reduction of 0 to $50 \%$ done at 1 hour before off block. Sector Koksy High. . . . . . . . . . . 80

8.4 Standard deviation arrival time prediction error sensitivity. . . . . . . . . 82 


\section{Acronyms}

$\begin{array}{ll}\text { ACC } & \text { Area Control Centre } \\ \text { AOBT } & \text { Actual Off Block Time } \\ \text { ATFCM } & \text { Air Traffic Flow \& Capacity Management } \\ \text { ATOT } & \text { Actual Take-off Time } \\ \text { CRCT } & \text { Collaborative Routing Coordination Tool } \\ \text { CSV } & \text { Comma-Separated Values } \\ \text { ECAC } & \text { European Civil Aviation Conference } \\ \text { EFD } & \text { ETFMS Flight Data } \\ \text { ETFMS } & \text { Enhance Tactical Flow Management System } \\ \text { ETI } & \text { Sector Entry Time } \\ \text { ETOT } & \text { Estimated Take-off Time } \\ \text { FIR } & \text { Flight Information Region } \\ \text { FPL } & \text { Filed Flight Plan } \\ \text { GA } & \text { General Aviation } \\ \text { IFPLID } & \text { Initial Flight Plan Identification } \\ \text { RMSE } & \text { Root Mean Squared Error } \\ \text { RPL } & \text { Repetitive Flight Plan } \\ \text { SESAR } & \text { Single European Sky Air Traffic Management Research } \\ \text { SSE } & \text { Sum of Squared Errors } \\ \text { XTI } & \text { Sector Exit Time }\end{array}$




\section{Chapter 1}

\section{Introduction}

Today there is a high amount of uncertainty in the sector traffic forecast. Flow managers anticipate for unforeseen traffic by increasing the forecasted maximum capacity threshold by more than $10 \%$. This has a number disadvantages, one of which is sub-optimal traffic flow. The objective of this thesis is to investigate sector demand predictability by; quantification of uncertainty between forecast and real traffic, and sensitivity analysis of predictability by improved departure time predictions.

Before commencing discussion about the chapter outline, analysis and conclusions expressed in this thesis are the ones of the author and EUROCONTROL does not take any liability on the opinions stated in this document. The study has been co-funded and supported by EUROCONTROL.

This chapter introduces the general area of interest of the project, and discusses relevance of the work at an academic \& industry level. Chapter 2 gives an concise overview of the work that has been already carried out by other academics in the area of predictability. Then chapter 3 gives a description on how trajectory predictions are made, and the logic behind the statistical and sensitivity analysis is discussed. The main work can be divided into statistical analysis and sensitivity analysis. Statistical analysis consists out of 3 parts. First quantification of departure time predictability is discussed in chapter 4. Interesting for Airport Managers are the number of flights, as function of departure time prediction error, to show how many flights depart within the departure slot tolerance window. Second, chapter 5 includes analysis of predictability in terms of sector entry time, sector occupancy count and sector entry count. Above metrics are plotted as function of look-ahead time to sector entry, to give an overview of the Flow Manager's perspective. Chapter 6 validates the obtained sector counts with an Eurocontrol software tool. Third, in chapter 7 the arrival time uncertainty is quantified, hereby the look-ahead time is defined as look-ahead until take-off in order to show uncertainty along the complete flight time horizon. In chapter 8 the sensitivity on arrival time \& sector demand predictability by improving departure time predictability is discussed. Chapter 9 discusses the impact of the key findings on predictability, and gives recommendations based on the work. Finally in chapter 10, the main results of the study are discussed, and recommendations for future work are made. 


\subsection{Prediction Uncertainty}

Currently in Europe, flow restrictions are applied to maintain safe workload levels for Air Traffic Controllers (ATC). Due to prediction uncertainty it occurs that more aircraft than predicted enter these flow restricted sectors, exceeding their capacities by more than $10 \%$, which is unsafe and regarded as an over-delivery. Investigation finds that this is caused by a lack of flight plan adherence; not flying the initial requested flight level, not departing at the initial filed off block time, arriving later or earlier than planned at the sector, or deviating horizontally from the original planned route [5]. In all reported over-deliveries during $2009,35 \%$ of the cases where due to flights that were not flying according to the initial filed flight level. Disadvantages of over-delivery for the network are wasted capacity in some sectors, lack of confidence in traffic forecasting tools, increased workload for the Air Traffic Controller which in turn causes stress and impairment of working conditions, thus putting safety at risk.

Capacity in the European air traffic network is typically described as the number of aircraft that may safely enter a given airspace volume over a given period. Sector capacity is often estimated using fast-time simulations that use controller workload models. For operational uses, capacity is typically measured in terms of sector entries per hour or sector occupancy for a given period of time. Where capacity is exceeded, Flow Management Positions are notified, and can decide to apply flow restrictions if necessary. Hereby the most constraining airport or sector determines the volume of traffic that can be handled for a given flow. Flow restrictions, known as 'Air Traffic Flow \& Capacity Management (ATFCM) regulations' or in this thesis referred to as 'regulations', function by delaying affected aircraft at the departure airport. A flight affected by a regulation receives a 'departure slot'. Prediction uncertainty results in regulations that are applied but are not needed, or regulations are applied longer than needed, increasing unnecessary delay for Airspace Users [5]. Prediction uncertainty causes regulations to be unsuccessful in fulfilling their intended function, which is to limit sector traffic. A lack of flight plan adherence causes prediction uncertainty. Lack of flight plan adherence at airports could be caused by; poor delay management and failure to adhere to the planned departure times. Major reasons of en-route non-adherence to the initial flight plan becomes evident in flight level changes or a direct routing. Reasons for pilots to request different flight levels could be caused by tailwinds/headwinds, payload changes, or the wish by airline operators to fly the optimized fuel trajectories where possible. Reasons for Air Traffic Controllers to give direct routes are to lower traffic volume, or to make up lost time for a flight. But level changes and direct routing cause approach sequence problems, ground handling timing issues, and flights to enter sectors upstream that did not anticipate for this traffic.

ATFCM traffic forecasts builds in assumptions about the prediction uncertainty. This forces the Air Navigation Service Providers to incorporate protective capacity reduction (capacity buffer) of $>10 \%$ to compensate for any unforeseen traffic volume change or traffic complexity change (see figure 1.1). Hence the declared capacities are $>10 \%$ lower than the real capacities. Aim is to improve predictions, consequently the delta between forecast and real traffic demand is considerably reduced, hence the Air Navigation Service Providers confidence in the forecast figures will raise and the capacity buffer can be stepwise reduced to ultimately $<3 \%$. 


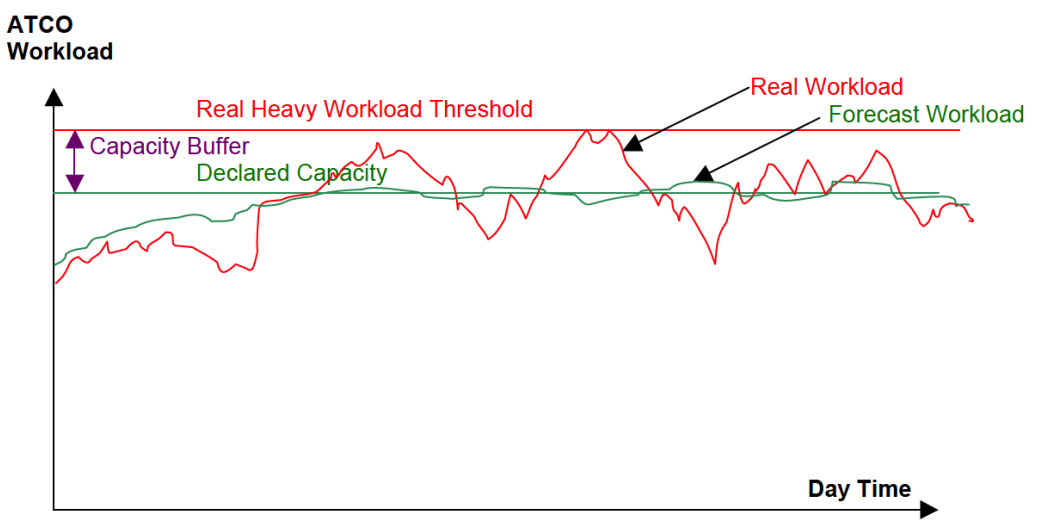

Figure 1.1: Forecast ATC workload versus real ATC workload (estimation) [6]

\subsection{Benefits of Improved Predictability}

One benefit of improved predictability is more capacity for planning, as better predictability leads to better pre-tactical decision making on scenarios and route restrictions. Decisions made on more accurate input data are therefore likely to reduce delays.

Better predictability should enable better decision making about whether a regulation is needed and for how long. In combination these two should lower the amount of ATFCM regulations thus lower delay.

One of the key tactical measures used to protect sectors from overload is level capping (limiting the highest flight level) of up-stream flights, so that they do not enter the sectors. Greater predictability is expected to reduce the need for such level capping and therefore contribute to greater vertical flight efficiency for aircraft thus saving fuel-costs for airlines.

Sector productivity is improved due to better predictability by better decision making about sector opening and closing times. During peaks of traffic, increased predictability is expected to reduce controller workload, as controllers are able to define better strategies in advance.

Greater predictability is expected to help flow managers to better understand the nature of the excess demand and make decisions that will maximize throughput whilst minimizing the risk of overloads, thus improving safety.

The Single European Sky ATM Research (SESAR) is a major pan-European industry led collective program for the modernization of the air traffic infrastructure. Aim for SESAR in the future are to move from airspace based operations towards trajectory based operations, where key terms are Shared Business Trajectories (SBT) and Reference Business Trajectories (RBT). The SBT is made widely available for ATM planning purposes to authorized users. The RBT represents an agreed 4D flight profile between Airspace Users and the Air Navigation Service Provider which takes account of route charges, approach sequencing, predictable arrival times for ground handling etc. In figure 1.2 the aim is to stepwise reduce uncertainty in the context of SESAR 2020. 


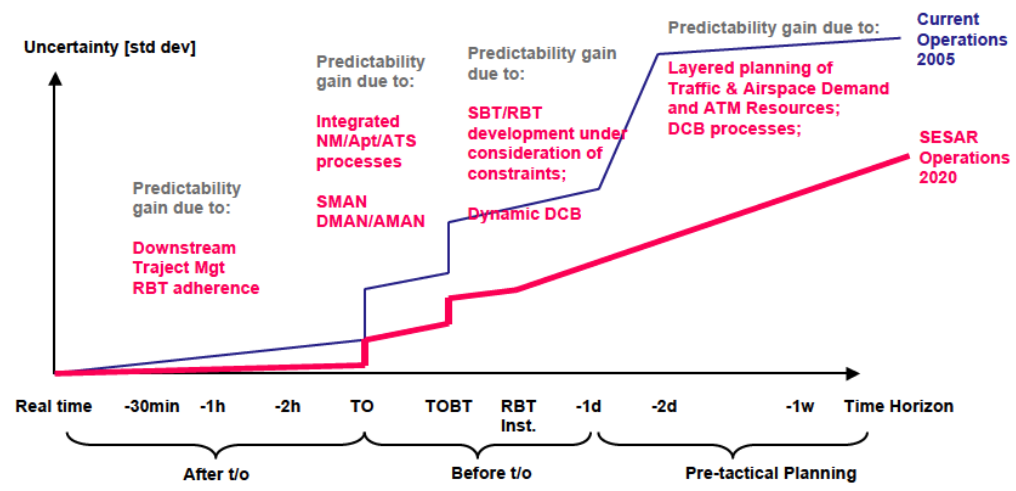

Figure 1.2: Trajectory prediction uncertainty over time horizon (estimation) [6].

\section{$1.3 \quad$ Research Objectives}

This thesis aims to bring more rigor to the body of knowledge by quantitative analysis of uncertainty for the European airspace. Main objective of this thesis is to investigate sector demand predictability by:

- Quantification of the uncertainty of sector entry time between forecast and real traffic;

- Quantification of the impact of trajectory deviations on the predictability of sector counts;

- Evaluation of the improvement in predictability by sensitivity analysis of improved departure time predictions.

To achieve this objective, an answer is sought to the questions: What is the accuracy of sector demand predictions? and What is the impact improved departure time predictions on sector demand predictability?

Scope of this thesis is not only sector demand predictability, but also departure \& arrival time predictability. Predictability for operational use is currently measured in terms of sector entries per hour or sector occupancy for a given time period. Predictability in terms of controller workload is not evaluated due to lack of data availability. Furthermore the thesis quantifies figures 1.1 and 1.2 based on real data.

\subsection{Individual Flight Example}

A red line throughout the thesis is a British Airways flight from London to Zurich, flying through Eurocontrol sector Koksy High (FL335 and above). This individual flight is used as example to explain more complex figures further on. The predicted and actual flight trajectory at 28th of June 2013 are shown in figure 1.3. Appendix A.1 shows a 4D trajectory prediction message. Relevant fields that are extracted from this message are timestamp (time at which the prediction is made), actual off block time (AOBT), actual 
take-off time (ATOT), sector entry time, and message event. Extracting all messages, and only relevant fields for this flight yields table 1.1. Every row is a prediction update. Look-ahead and prediction error is calculated with equations 3.1 and 3.2. Events triggering a new update could be IFP (initial flight plan filing), PTX (periodic transmission, a "reminder" if no new predictions are send for a while), FSA (first system activation, indicating take-off event), or CPR (correlated position report, an update by radar surveillance data).

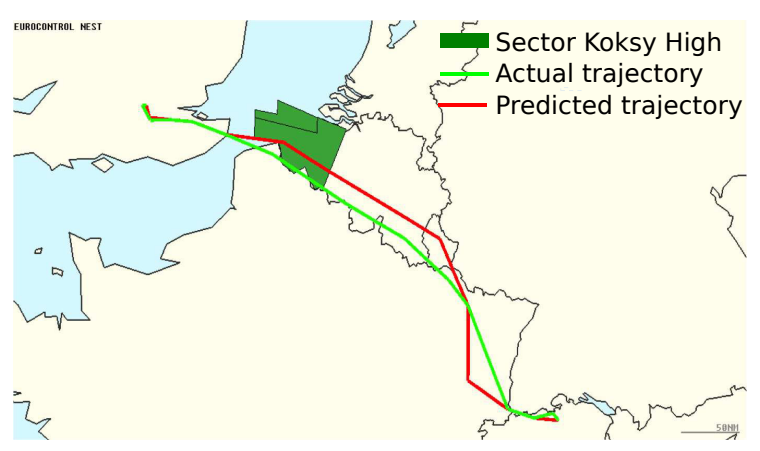

Figure 1.3: Geographic top-down view of predicted \& actual trajectory for example flight [3]
Table 1.1: Prediction for individual example flight.

\begin{tabular}{|c|c|c|c|c|c|c|}
\hline $\begin{array}{l}\text { Look- } \\
\text { ahead } \\
\text { time to } \\
\text { sector } \\
\text { entry }\end{array}$ & $\begin{array}{l}\text { Sector } \\
\text { entry } \\
\text { time } \\
\text { prediction } \\
\text { error }\end{array}$ & $\begin{array}{l}\text { Time- } \\
\text { stamp }\end{array}$ & $\begin{array}{l}\text { Off } \\
\text { block } \\
\text { time }\end{array}$ & $\begin{array}{l}\text { Take } \\
\text { off } \\
\text { time }\end{array}$ & $\begin{array}{l}\text { Sector } \\
\text { entry } \\
\text { time }\end{array}$ & $\begin{array}{l}\text { Message } \\
\text { event }\end{array}$ \\
\hline 01:54 & $00: 14$ & $10: 10$ & 11:00 & $11: 20$ & $11: 50$ & IPF \\
\hline 01:09 & $00: 14$ & $10: 55$ & 11:00 & $11: 20$ & 11:50 & PTX \\
\hline $00: 56$ & $00: 12$ & 11:08 & 11:00 & $11: 20$ & $11: 52$ & PTX AOBT \\
\hline $0 \overline{0}: \overline{4} 6$ & $0 \overline{0}: \overline{10}$ & $11: 18$ & $1 \overline{1}: 02$ & $1 \overline{1}: \overline{2} \overline{2}$ & $\overline{1} \overline{1}: \overline{5} 4$ & $\overline{\mathrm{P}} \overline{\mathrm{T}} \overline{\mathrm{X}}$ \\
\hline $00: 36$ & 00:08 & $11: 28$ & 11:04 & $11: 24$ & $11: 56$ & PTX ATOT \\
\hline $00: 28$ & $0 \overline{0}: \overline{0} \overline{7}$ & $11: 36$ & $11: 10$ & $1 \overline{1}: 30$ & $\overline{1} \overline{1}: \overline{7}$ & $\overline{\mathrm{F}} \overline{\mathrm{S}} \overline{\mathrm{A}}$ \\
\hline $00: 26$ & 00:07 & 11:38 & 11:10 & $11: 30$ & 11:57 & CPR \\
\hline 00:14 & 00:02 & 11:50 & 11:10 & $11: 30$ & $12: 02$ & CPR \\
\hline 00:04 & 00:01 & 12:00 & 11:10 & $11: 30$ & 12:03 & CPR \\
\hline 00:00 & 00:00 & 12:04 & 11:10 & 11:30 & 12:04 & CPR \\
\hline
\end{tabular}

Look-ahead time is calculated by equation 3.1. Figure 1.4 shows how look-ahead is defined starting from figure 1.4a. Here prediction error as function of timestamp is plotted in table 1.1. Now flipping timestamp from left to right yields figure 1.4b. Finally replacing timestamp with look-ahead to sector entry yields figure $1.4 \mathrm{c}$ 


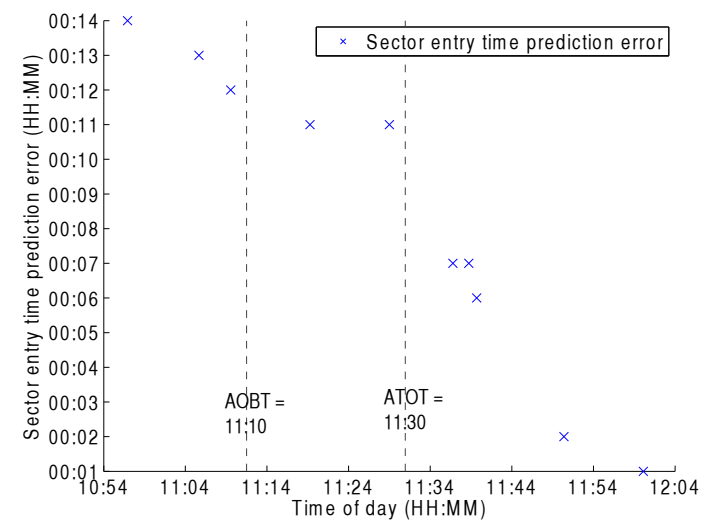

(a) Time of day from early to late

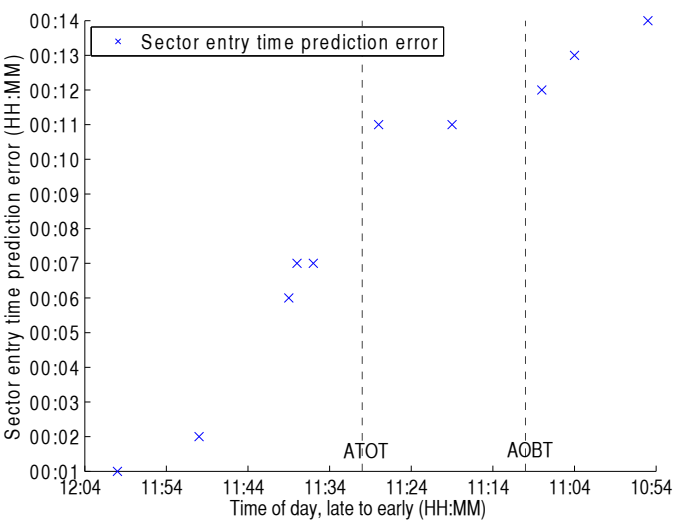

(b) Flipping time of day from left to right, late to early

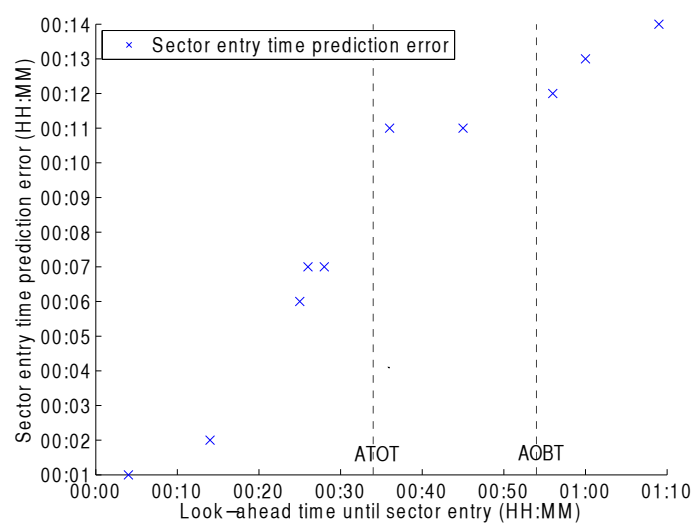

(c) Replacing time of day with look-ahead time to sector entry

Figure 1.4: Example of look-ahead time definition, for individual flight. 


\section{Chapter 2}

\section{Literature Review}

This chapter presents a concise review of papers that are relevant to the thesis objective; the investigation of sector demand predictability. Uncertainty factors as regulations, sector traffic type, flight type and weather are discussed. Most findings presented on prediction error are only available for U.S. airspace, caution must be applied, as the findings might not be transferable to the European airspace. This chapter is concluded with the key findings of the literature review.

\subsection{Factors Affecting Sector Demand Predictability}

Table 2.2 shows factors that affect sector demand predictions, obtained from relevant articles. [10] conducts research on the two most critical sector demand error sources; (1) prediction of departure time, and (2) prediction of ATFCM regulations and ATC procedures. These two error factors are ranked highest in terms of their influence on sector demand prediction based on interviews with air traffic controllers and researches. [12] identified and analyzed three variables that have the strongest effects on uncertainty distributions; (1) look-ahead time, (2) prediction peak count and (3) sector traffic type. [11] does not rank uncertainty sources but investigates prediction performance by weather type, flight plan submission, regulation and flight type.

Table 2.1: Sample size and analyzed metrics for relevant articles.

\begin{tabular}{llll} 
Reference & Sample time & Sample airspace & $\begin{array}{l}\text { Analyzed prediction } \\
\text { performance metrics }\end{array}$ \\
\hline$[12]$ & 286 days & 754 U.S. sectors & peak count \\
\hline$[9]$ & 30 days & Spanish FIR & sector entry time \\
\hline$[10]$ & 5 days & 2 U.S. ACC's & $\begin{array}{l}\text { sector occupancy count, } \\
\text { sector entry time }\end{array}$ \\
\hline$[11]$ & 4 days & 2 U.S. ACC's & $\begin{array}{l}\text { sector entry time, } \\
\text { hit rate }\end{array}$
\end{tabular}

Table 2.1 shows sample time, sample airspace, and studied prediction performance metrics used in the articles that are discussed in this section. Take note that prediction error for the reviewed papers is calculated by the "actual time" minus "predicted time". 
Table 2.2: Factors affecting sector demand predictions.

\begin{tabular}{|c|c|c|}
\hline Remarks & Factors & Examples \\
\hline \multirow{10}{*}{$\begin{array}{l}\text { Error sources sorted } \\
\text { by level of influence } \\
\text { on sector demand } \\
\text { prediction, high to low. } \\
{[10]}\end{array}$} & Departure time prediction & $\begin{array}{l}\text { Abnormal surface events, } \\
\text { unavailable gates }\end{array}$ \\
\hline & $\begin{array}{l}\text { Prediction of ATFCM initiatives } \\
\text { and ATC actions }\end{array}$ & $\begin{array}{l}\text { Non-standard procedures, } \\
\text { style and preferences } \\
\text { of controllers }\end{array}$ \\
\hline & Horizontal route prediction accuracy & \\
\hline & Vertical route prediction accuracy & \\
\hline & Flight speed prediction accuracy & \\
\hline & Changing airspace adaption data & \\
\hline & Weather and wind forecast accuracy & \\
\hline & Accuracy of surveillance data & \\
\hline & Flight technical and operational errors & \\
\hline & Accuracy of trajectory models & \\
\hline \multirow[t]{4}{*}{$\begin{array}{l}\text { Factors affecting } \\
\text { sector demand } \\
\text { predictions } \\
{[12]}\end{array}$} & Airspace & $\begin{array}{l}\text { Sector altitude class, } \\
\text { primary traffic type } \\
\text { (departures, arrivals, } \\
\text { en route, mixed), ACC }\end{array}$ \\
\hline & Time & $\begin{array}{l}\text { Day of week, hour of day, } \\
\text { time of year (season) }\end{array}$ \\
\hline & Prediction & $\begin{array}{l}\text { Look-ahead time } \\
\text { Peak count }\end{array}$ \\
\hline & Weather & Severe weather, jet stream \\
\hline \multirow{4}{*}{$\begin{array}{l}\text { Investigated factors affecting } \\
\text { sector demand } \\
\text { predictions } \\
{[11]}\end{array}$} & Weather & Good or bad \\
\hline & Flight plan submission & Scheduled or Filed Flight Plan \\
\hline & Regulation & Filed or regulated departure times \\
\hline & Flight type & Commercial, GA or military flights \\
\hline $\begin{array}{l}\text { Factor affecting FIR } \\
\text { demand prediction } \\
{[9]}\end{array}$ & Traffic type & Departing or arriving \\
\hline
\end{tabular}

\subsubsection{Flight Plan Filing Time}

Prediction performance for predictions of U.S. flights based on scheduled and filed flight plans is analyzed [11]. Scheduled flight plans are based on the Official Airline Guide (OAG). OAG data can be inaccurate compared to filed flight plans, as OAG produces future commercial airline schedules once a month, and filed flight plans are normally submitted 90 minutes before planned departure time.

Figure 2.1a and table 2.3 show magnitude and variability of absolute sector entry prediction error for first filed flight plans and last scheduled flight plans, as a function of time to departure.

Two-sample t-tests (hypothesis testing) were conducted to determine if observed difference between filed and scheduled flight plans (for sector entry time error and departure time error) are significant or due to natural variation. Concluded is that filed flight plans do not provide better prediction performance than scheduled flight plans.

Hit rate is the fraction of flights predicted to enter a sector, of the flights that really enter the sector. [11] states that hit rate is significantly greater for filed flight plans for more than $65 \%$ of the predeparture look-ahead time on the good weather days and more than $88 \%$ on the bad weather days. Vertical profiles, for scheduled and filed plan trajectories, are expected to be similar because estimated departure times for scheduled and filed flight plans are similar. Suggested is that the observed difference in hit rate is due to differences in the specified lateral route for corresponding scheduled and filed flight plans. 
Table 2.3: Mean and standard deviation for sector entry time prediction error [11]

\begin{tabular}{|c|c|c|c|c|c|c|c|}
\hline \multirow[b]{2}{*}{ Metric/scenario } & \multicolumn{7}{|c|}{ Predeparture time, min } \\
\hline & 0 & 30 & 60 & 90 & 120 & 180 & 240 \\
\hline Mean $S^{a} B W x^{b}$ & 5.24 & 12.19 & 8.11 & 10.03 & 18.29 & 13.56 & 22.86 \\
\hline StDev S BWx & 3.19 & 9.48 & 7.46 & 9.85 & 16.21 & 13.99 & 17.38 \\
\hline Mean $S G W x^{c}$ & 4.83 & 12.48 & 9.46 & 9.11 & 15.91 & 9.41 & 17.72 \\
\hline StDev S GWx & 5.15 & 8.75 & 7.90 & 8.80 & 14.76 & 9.95 & 14.17 \\
\hline Mean $\mathrm{F}^{\mathrm{d}} \mathrm{BWx}$ & 14.06 & 11.88 & 7.24 & 9.89 & 17.26 & 12.67 & 31.21 \\
\hline StDev F BWx & 15.94 & 7.83 & 6.01 & 9.34 & 15.44 & 12.99 & 22.06 \\
\hline Mean F GWx & 9.99 & 11.42 & 8.23 & 8.53 & 14.73 & 9.36 & 19.41 \\
\hline StDev F GWx & 7.47 & 7.42 & 6.14 & 8.01 & 14.18 & 10.12 & 14.93 \\
\hline
\end{tabular}

${ }^{\mathrm{a}}$ Scheduled flight. ${ }^{\mathrm{b}}$ Bad weather day. ${ }^{\mathrm{c}}$ Good weather day. ${ }^{\mathrm{d}}$ Filed flight.
Table 2.4: Absolute mean and standard deviation for departure time prediction error [11]

\begin{tabular}{|c|c|c|c|c|c|c|c|}
\hline \multirow[b]{2}{*}{ Metric/scenario } & \multicolumn{7}{|c|}{ Predeparture time, min } \\
\hline & 0 & 30 & 60 & 90 & 120 & 180 & 240 \\
\hline Mean $S^{a} B W x^{b}$ & 5.34 & 11.57 & 8.85 & 14.34 & 21.13 & 17.53 & 37.80 \\
\hline StDev S BWx & 6.66 & 9.88 & 9.75 & 13.25 & 18.59 & 15.67 & 10.11 \\
\hline Mean $\mathrm{S} \mathrm{GWX}^{\mathrm{c}}$ & 4.73 & 11.40 & 7.66 & 10.36 & 18.30 & 11.95 & 39.89 \\
\hline StDev S GWx & 4.48 & 8.54 & 7.53 & 11.14 & 16.91 & 13.35 & 12.66 \\
\hline Mean $\mathrm{F}^{\mathrm{d}} \mathrm{BWx}$ & 10.44 & 10.97 & 8.84 & 13.66 & 20.07 & 16.89 & 41.23 \\
\hline StDev F BWx & 8.54 & 8.83 & 9.72 & 13.38 & 17.41 & 15.62 & 12.36 \\
\hline Mean F GWx & 7.82 & 9.65 & 7.69 & 10.05 & 17.47 & 11.80 & 41.24 \\
\hline StDev F GWx & 7.98 & 6.09 & 7.07 & 10.60 & 16.42 & 13.38 & 12.95 \\
\hline
\end{tabular}

Half of all filed commercial flight plans, for the 2 examined ACC's in U.S. are filed 90 minutes before departure [11]. Half of all filed flight plans are filed 9 hours before entry into the Spanish FIR [9]. When the flight plan filling occurs the routes for the flights are known more accurately, however the flying time to sectors is no more accurate [11].

\subsubsection{Regulations}

Commercial flights with filed flight plans and flights with regulated departure times during bad weather are compared [11]. Flights usually depart at or after their planned times. Figure 2.1b shows and statistical tests confirm that sector entry error is greater for regulated flights at predeparture look-ahead times longer than 1 hour. Departure time error is significantly greater for regulated flights only at 90-195 minutes before departure.

Sometimes U.S. ground delay programs are canceled allowing regulated flights to depart before their regulated departure times. Sector entry time error is investigated for regulated flights that did and did not have regulated departure times at take-off during bad weather. Absolute sector entry time predictions are more accurate for regulated flights that still had a regulated departure time at take-off, for 15 to 165 minutes before departure. Figure 2.2 also shows that for regulated flights with no regulation at take-off, the prediction tool used in the U.S. transport system (CRCT), tends to predict that the flights enter sectors later than they actually do in predictions made up to 2.5 hours before departure (positive error means flights are delayed). This is abnormal because CRCT typically predicts that flights enter sectors earlier than they actually do. CRCT hit rate prediction performance for regulated flights is better for flights with regulated take-off time at take-off opposed to flights with no regulated take-off time at take-off. For these flights the predicted routes are a more accurate representation of intent than for flights with no regulated take-off time at take-off.

5 sector demand prediction error sources in the U.S. airspace system related to ATC procedures and ATFCM regulations are identified [10]:

- Aircraft sector entry times that are late due to conflict resolution by path stretching or vectoring. 


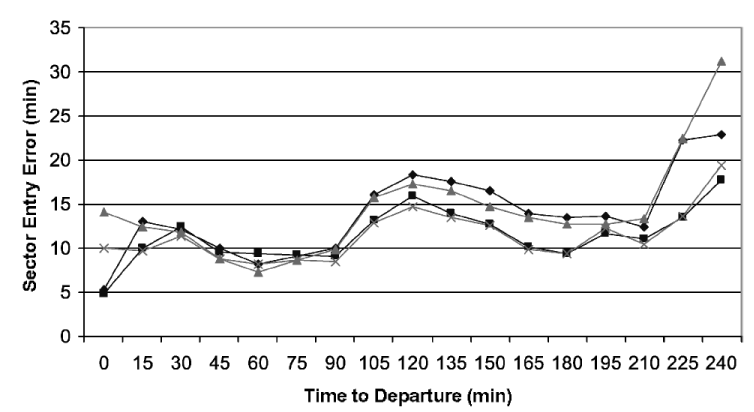

(a) With; • scheduled, bad weather; • scheduled, good weather; $\Delta$ filed, bad weather; and $\mathrm{x}$ filed, good weather. [11]

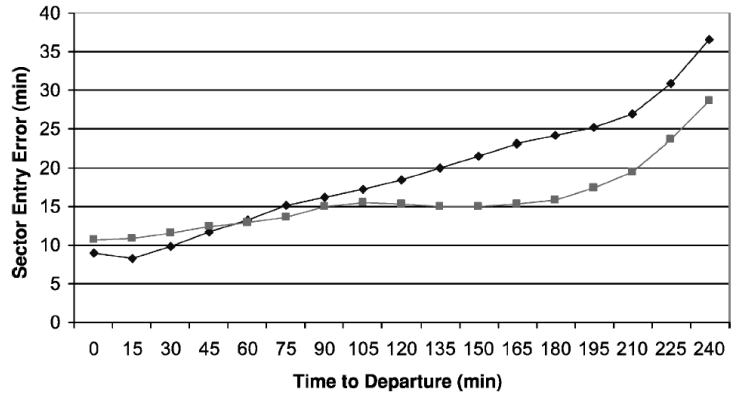

(b) - regulated flights and • filed flights [11]

Figure 2.1: Absolute sector entry time prediction error.

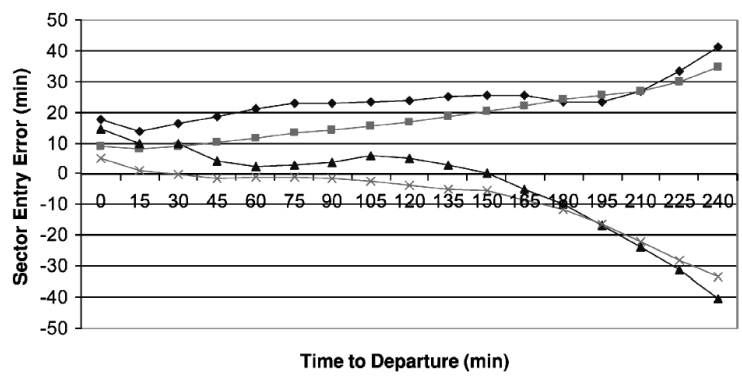

Figure 2.2: Sector entry time prediction error for flights with and without regulation: • no regulation/take-off mean absolute; - regulation/take-off mean absolute; $\Delta$ no regulation/take-off; and $\mathrm{x}$ regulation/take-off. [11]

- Circular holding used for en-route aircraft when upstream sector or airport capacity is too high.

- Direct to routes, aircraft flying shorter routes than predicted.

- SOP (Standard Operating Procedures) or LOA's (Letters of Agreement). These establish rules and restrictions that limit and affect trajectory predictions.

Table 2.5 shows mean \& standard deviation of the prediction error of 3 different time instances in the flight time horizon; take-off time, 30 minutes after take-off, and 1 hour after take-off. This is calculated for all daily flights in the European airspace (approximately 30 000). Prediction error is calculated by subtracting "actual time" minus "predicted time at initial flight plan". Distinctions are made between busy day, normal day, regulated, and non-regulated flights (4 scenario's). On the busy day there are approximately 6000 regulated flights, on the normal day 1700 regulated flights. This suggests that there is a linear increase of standard deviation after take-off: 1 minute per hour flown. Variability (standard deviation and mean) of departure time prediction error for regulated flights is smaller than non-regulated flights. 


\subsubsection{Predeparture Components}

Figure 2.3 illustrates the contribution of predeparture errors to total predictive errors on a good weather day [11] . Sector entry time prediction error is determined for proposed (not yet departed) commercial flights at various times before departure and for active (airborne) flights for various flight times to the sector. The total prediction error of sector entry time is subtracted by the prediction error for active flights to get the predeparture component of the prediction error. The predeparture component dominates the sector prediction error on good and bad weather days, the relative contribution of predeparture uncertainty to the total prediction error is slightly less on bad weather days than good weather days.

Table 2.5: Mean and standard deviation for 'time over' prediction error; $\mathrm{R}=$ regulated, $\mathrm{B}=$ busy day, Normal $=$ normal day, Non $=$ non regulated [7]

\begin{tabular}{llll} 
& Time after & departure $(\mathrm{min})$ & \\
\cline { 2 - 4 } Metric/scenario & 0 & 30 & 60 \\
\hline Mean R B & 0.9 & 1.0 & 1.3 \\
StDev R B & 7.7 & 8.2 & 8.7 \\
Mean R Normal & 0.3 & 0.1 & 0.1 \\
StDev R Normal & 6.4 & 6.9 & 7.3 \\
Mean Non B & 4.8 & 4.6 & 4.6 \\
StDev Non B & 13.3 & 13.7 & 14.0 \\
Mean Non Normal & 2.0 & 1.5 & 1.4 \\
StDev Non Normal & 11.4 & 12.0 & 12.4 \\
\hline \hline
\end{tabular}

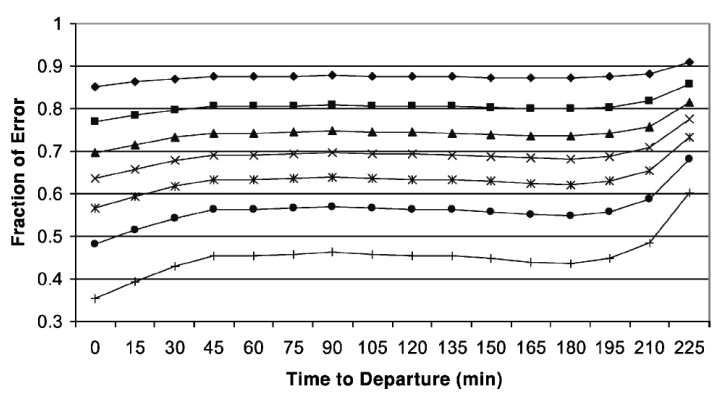

Figure 2.3: Predeparture component of sector entry time prediction error for good weather: 0 $\min$ to sector; +180 min to sector, increments of $30 \mathrm{~min}$ in-between.

Figure 2.4 illustrates the absolute sector occupancy count error for an U.S. ACC close to Chicago O'Hare airport, over the time of day. Data indicates that the sector count error is dominated by proposed flights instead of active flights. Noticeable is the decrease of sector count error at 16:00.

There is a caveat to sector occupancy count predictions. It fails to address how individual flights contribute to those counts. For example, an aircraft may be predicted to occupy a sector that never actually enters. Another aircraft, not predicted to enter the sector does in fact enter at around the same time the first aircraft was predicted to do so. The sector count prediction for this particular sector would have been correct, although two aircraft actually occupied unpredicted sectors [10].

\subsubsection{Sector Traffic Type}

A distinction is made between four traffic categories based on the predominant type of traffic passing through the sector; (A) arrival, (D) departure, (E) en-route and (M) mixed [12]. The considered traffic consists of proposed and active flights. Figure 2.5a shows mean and standard deviation of mixed sector peak prediction error. Note that the horizontal axis indicates predicted peak count (maximum sector occupancy count) and the vertical axis indicates the mean \& standard deviation of the peak prediction error; predicted 


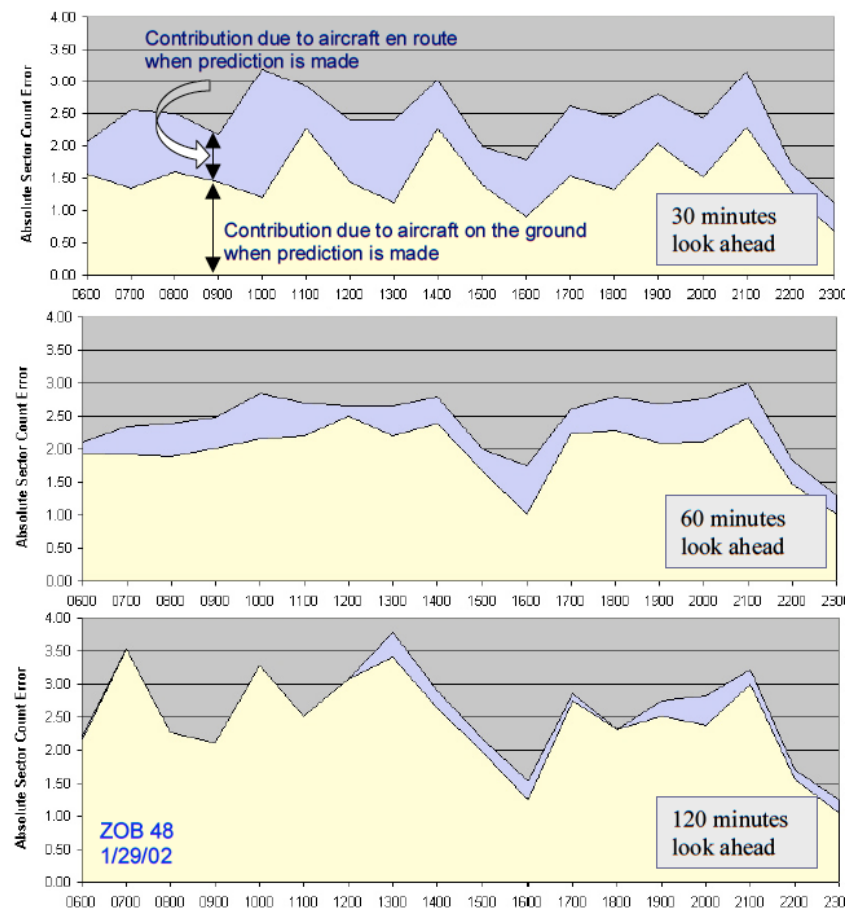

Figure 2.4: Sector occupancy count prediction error for active vs. proposed flights at different times of the day. [10]

peak value minus actual peak value. Peak prediction error, contrary to the calculation of prediction error for the other papers, is defined as predicted peak count minus actual peak count. This means that a negative mean prediction error is an under-prediction. For predicted peak count of 4 upward, the mean prediction error steadily increases with increasing predicted value. No explanation is given for the increase in mean prediction error from a peak count of 4 and upward predicted flights.

Figure 2.5b shows mean and standard deviation of peak count error for a 1 hour lookahead time, categorized by sector traffic type. Departure sectors involve a much greater proportion of pre-departure flights than for other sector types. Because departure time predictions are highly uncertain, predictions for departure sectors are more uncertain. Similarly, predictions are more accurate for arrival sectors since a higher proportion of involved flights are airborne at 1 hour before entry.

Figure 2.6a and figure 2.6b shows the cumulative distribution function (CDF) for 4 lookahead times and four traffic type sectors. Each point (y-axis value) on the CDF indicates the probability that the prediction error is equal to or less than the corresponding $\mathrm{x}-$ axis value. This means that an unbiased prediction would have a CDF value of 0.5 for a prediction error of zero, and the steeper the slope of the CDF, the more precise the prediction.

Flight departing in the Spanish FIR (Flight Information Region) typically tend to be earlier than planned. Flights arriving at the FIR tend to be later than indicated in the initial flight plan. Flights arriving at Barcelona FIR are less delayed than flights arriving at the bigger Spanish Peninsular FIR [9]. 


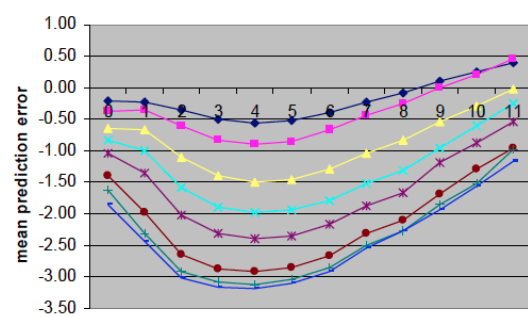

predicted flights

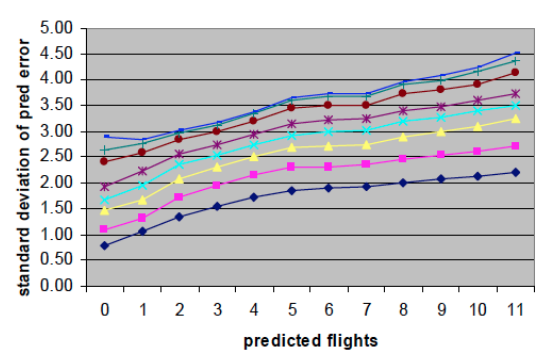

(a) For mixed sectors by look-ahead time $(\min )[12]$.
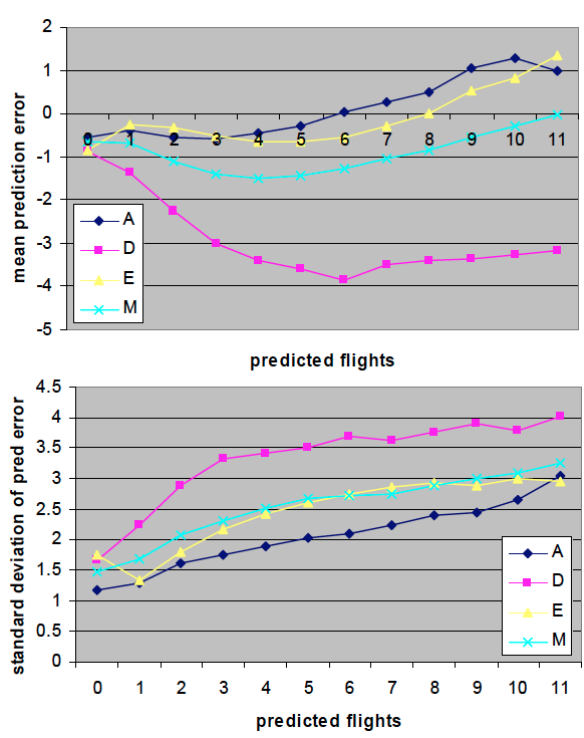

(b) For 1 hour look-ahead for sector type: (A) arrival, (D) departure, (E) en-route and $(\mathrm{M})$ mixed [12]

Figure 2.5: Mean and standard deviation of peak count prediction error.

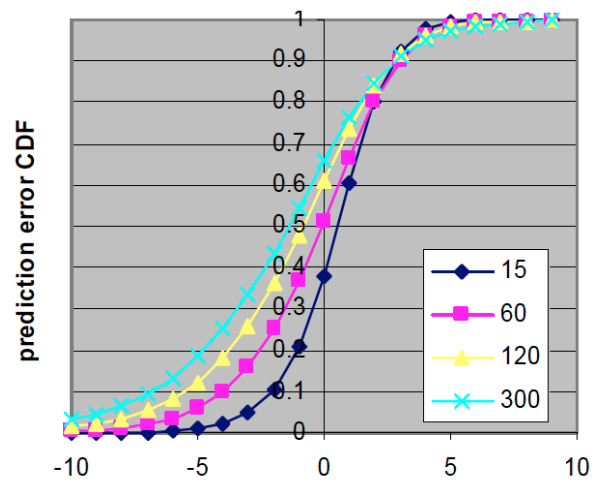

(a) For mixed sectors for different look-ahead times [12]

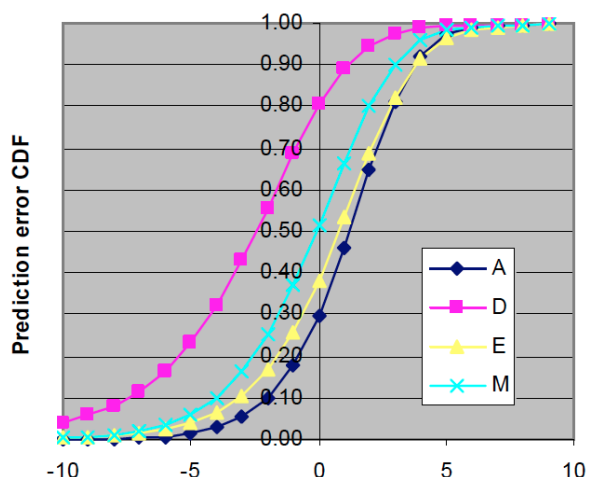

(b) For 1 hour look-ahead for different sector types: (A) arrival, (D) departure, (E) enroute and $(\mathrm{M})$ mixed [12]

Figure 2.6: Peak count cumulative distribution function.

\subsubsection{Flight Type}

Figure 2.7a shows prediction accuracy for commercial, general aviation (GA), and military flights [11]. Sector entry error is smallest for commercial flights, and estimated departure time error is also smallest for these flights. The sample of military flights and sample of GA flights on bad weather dates is relatively small, making prediction performance metrics for those flights more susceptible to outlier influences. Hit rate is higher on good 
weather days for each aircraft type, and suggests that flight plan routes more accurately reflect the path aircraft take on good weather days.

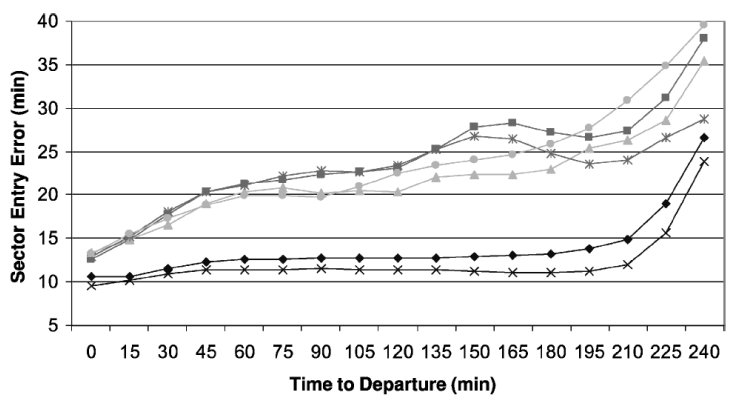

(a) With; • commercial, bad weather; - GA, bad weather; $\triangle$ military, bad weather; $\mathrm{x}$ commercial, good weather; $)($ GA, good weather; and • military, good weather. [11].

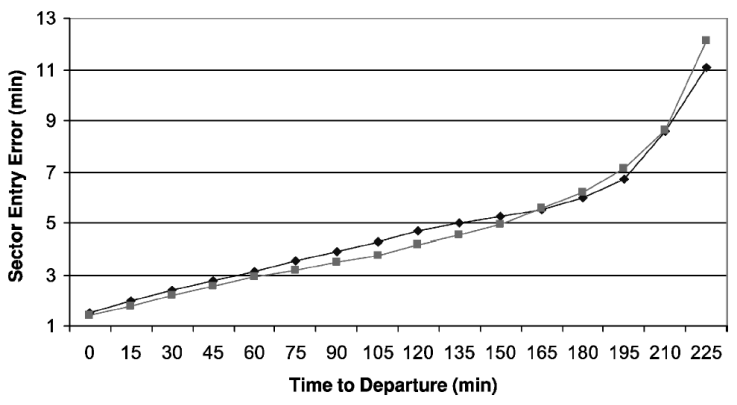

(b) With; - good weather and • bad weather $[11]$

Figure 2.7: Sector entry time prediction error for different flight types \& weather types.

\subsubsection{Weather}

Figure 2.7b shows sector entry prediction error during good and bad weather. It can be seen that prediction accuracy during good weather is slightly better than bad weather from 0 to 165 minutes before departure.

Taxi times during the time period when de-icing operations took place, are on average more than double the taxi time during days when there was no de-icing for Detroit Metropolitan Airport [10].

\subsubsection{Operational Impact}

5 experts have been interviewed from Air Navigation Service Providers with sectors that have high demand, high complexity, and high seasonal variation [5] . Results from the interviews in terms operational impact are:

- Unpredictable flight levels creates $70-90 \%$ of the problems on operations.

- Unpredictable lateral deviations creates $0-5 \%$ of the problems on operations.

- Unpredictable time deviations creates $5-30 \%$ of the problems on operations.

[8] has given theoretical models of the relationship between sector occupancy count prediction error variance (under regulation) and entry time prediction error variance. Occupancy count standard deviation is fairly insensitive to changes in entry time standard deviation for large values of entry time deviation. For example, a $50 \%$ reduction in entry time standard deviation (from 10 minutes to 5 minutes) results in a $3 \%$ increase in the capacity of a busy sector. 


\subsection{Conclusions}

The literature study examined papers relevant to the research question: What is the accuracy of sector demand predictions? Findings show that the following factors have a deteriorating effect on prediction accuracy:

- Non-regulated flights at and after departure, as opposed to regulated flights at and after departure (figure 2.2 and figure 2.3).

- Not yet airborne flights as opposed to airborne flights (figure 2.4)

- Sectors with departing flights as opposed to sectors with arriving and en-route flights (figure 2.6a).

- Military and general aviation flights as opposed to commercial flights (figure 2.7a).

Section 2.1 discusses prediction performance metrics, and relevant papers are mostly about the U.S. airspace. Departure time prediction error is found to be the major factor that impacts sector entry error. Caution must be applied in assuming that these metrics are the same for sectors in the European airspace. Flights typically depart at or after their planned departure times, and if flights are delayed due to regulations, flights increase their cruise speed to recover lost time on the ground. No literature has been found on improved departure time predictions relative to sector demand predictability. 


\section{Chapter 3}

\section{Modelling Framework}

This chapter examines the logic behind the statistical and sensitivity analysis. Section 3.1 describes how trajectory prediction are made. Section 3.2 explains the logic to calculate relevant metrics from the trajectory predictions. Then in section 3.3 the equations used to calculate relevant metrics are given. Finally in section 3.4, the general principle to obtain confidence intervals is stated, this interval shows how confident we are that the true parameter value is in the reported interval.

\subsection{Trajectory Predictions}

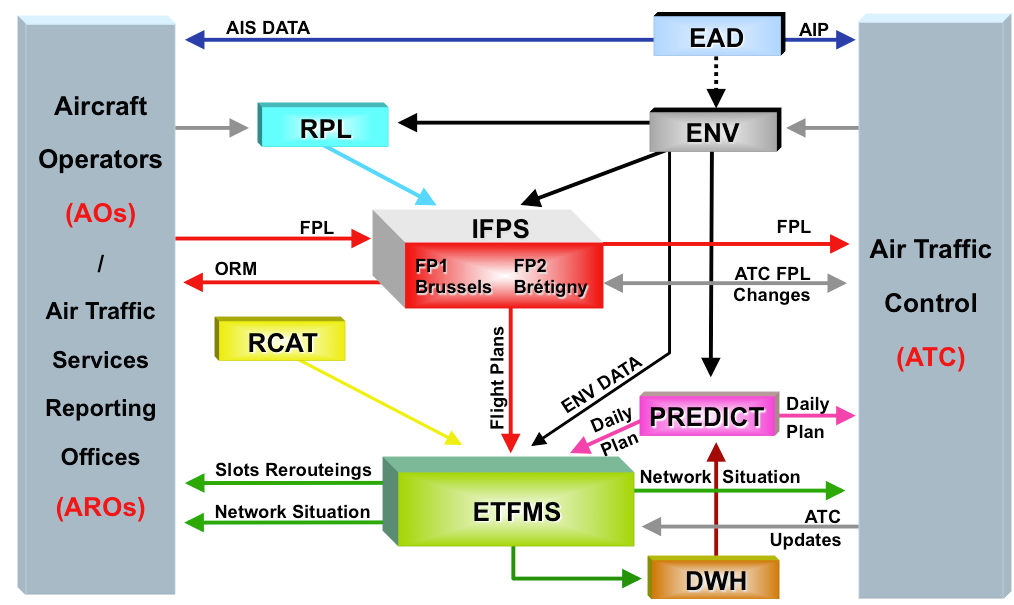

Figure 3.1: Main data flows of ETFMS [1].

The Enhanced Tactical Flow Management System (ETFMS) calculates traffic demand in every European airspace sector using flight plans, and allocates departure slots. Figure 3.1 shows main data flows to and from the ETFMS.

The ETFMS receives the following input; 
- Flight plans (FPL and RPL) from Aircraft Operators. These are received and processed by the Integrated Flight Plan System in Haren, and then send to the ETFMS .

- Environmental data (ENV) e.g. meteorological updates. Wind speed and wind direction are used in the flight profile calculations.

- ATFCM regulations.

- Route Catalogue (RCAT). List of all routes between a given city pair.

- Pre-Tactical System (PREDICT). System that compares historic demand with forecasted capacities and sector configurations to predict the need for the following day's ATFCM regulations.

- Airborne aircraft positions. Mainly radar surveillance data send by Air Navigation Service Providers.

And the ETFMS generates the following output;

- Entry \& occupancy count. See figure 3.2 .

- Departure slot messages for regulated flights. Flights that are planning to fly into sectors were capacity is exceeded, are delayed at the departure airport by regulated departure times.

- ETFMS Flight Data file (EFD).

- Archived flight lists in the data warehouse (DWH).

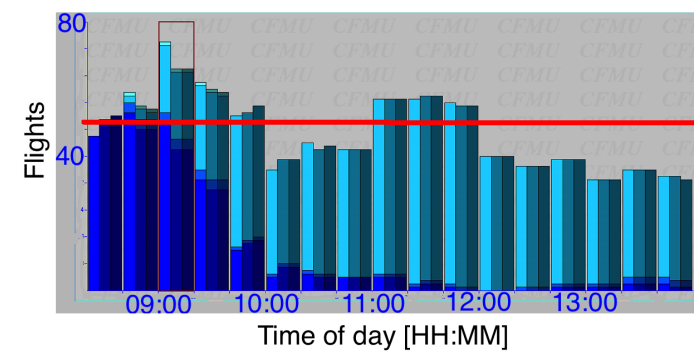

(a) Sector Entry Count.

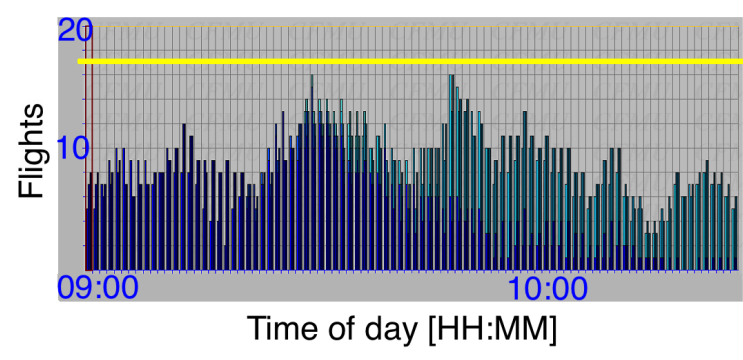

(b) Sector Occupancy Count.

Figure 3.2: Sector count prediction example from the Flow Manager's perspective [3].

The output of the ETFMS allows traffic predictions to be made, these predictions are made in terms of sector entry count (hourly flight entries) and sector occupancy count (flight occupancies for a given time period). These counts are shown in figure 3.2, displayed from the perspective of the flow manager. Where predicted capacity is exceeded, the bar is larger than the red/yellow line, and flow management controllers are able to make decisions whether to apply flow restrictions.

Count predictions are based on the ETFMS output data known as an EFD file. This data contains 4D flight profile predictions and is sent to Air Navigation Service Providers and Aircraft Operators via a dedicated network. A $4 \mathrm{D}$ flight profile prediction update is henceforth called prediction update. ETFMS prediction updates happen for significant flight events. Flight events that can trigger a prediction update can be the reception of 
flight plan messages, meteorological data updates, radar surveillance updates, etc. An example of such a radar surveillance data prediction update is given in appendix A.1. Per flight, there are approximately 60 prediction updates. A radar surveillance data prediction update only occurs if the flights exceeds the predicted trajectory thresholds which are; 300 seconds time deviation, 3 flight levels vertical deviation, or 5 nautical miles horizontal deviation. EFD files are used as input for the analysis in this thesis.

\subsection{Explanation of the Data Extraction Code}

This section elaborates on the logic of obtaining sector occupancy count from the EFD file. Then the logic behind departure time prediction improvement as done in the sensitivity analysis, is discussed. Although only sector occupancy count is discussed, the same logic can be used to obtain arrival times, departure times, sector entry times, and sector entry count. The departure time prediction improvement code can be inserted in the code of the above mentioned metrics, to obtain altered times.

\section{Sector Occupancy Count}

The flow diagrams in figure 3.3 can be divided in two scripts; Powershell and Matlab. First the Powershell script extracts the relevant data from the EFD file and gives the output in a CSV (comma-separated values) file. Every line of the EFD file is read $( \pm$ $1^{*} 10^{6}$ lines) and output is written if an EFD message (appendix A) meets the following conditions; sector entry time is on the 'current day', and the airspace profile contains the 'regarded sector'. If these conditions are met, the following fields are extracted from the message; timestamp (time of the prediction message), event triggering the message, flight plan identification, estimated off block time, actual off block time, take-off time, airport of departure, model (non-regulated non-airborne prediction, regulated non-airborne prediction, airborne prediction), sector entry time, sector exit time, taxi time. 'Current day' is the day of interest. Predictions for sector entry on the 'current day' are also made on the 'current day -1 day' and at the 'current day +1 '. This means that data has to be extracted from 3 days, the total computational time is 6 hours.

Second Matlab imports the CSV file and converts the dates/times to serial dates/times. Conversion is done to perform algebraic operations on times. Then the following differentiations are made; prediction message is made after or before take-off, flight is regulated or non-regulated, and prediction message is made after or before sector entry. A matrix is constructed, were each column is a 10 minute time of day window, ranging from '00:00' current day to 00:00 'next day'. If a flight is occupying the sector for a given window, the flight is displayed in that column. This is done for all prediction updates for all flights. Counting the number of unique flights per column, and including only latest known prediction updates before a specific time of day, yields the predicted count for a given time of day window. Actual count is obtained in similar fashion, but now only prediction updates after sector entry are included. Predicted and actual count are explained more elaborately in sections 5.3.3. Count error is elaborated in section 5.3.6. Computational time for this Matlab script is 40 minutes. 


\section{Departure Time Prediction Improvement}

Figure 3.4 shows the flow diagram of steps to improve departure time predictions, as done in the sensitivity analysis (chapter 8). This section can be placed between the following blocks in the sector occupancy count flow diagram in figure 3.3; 'Get flights ... at departure' and 'Construct table ... per window'. For regulated flights, the latest regulated take-off time before the actual take-off is extracted. For non-regulated flights, the estimated take-off time at off block, or at 1 hour before off block, is extracted. These regulated take-off and estimated take-off times are required to calculate the 'time shift' as explained in section 8.2. Altered times are calculated by subtracting the 'timeshift' from the unaltered times.

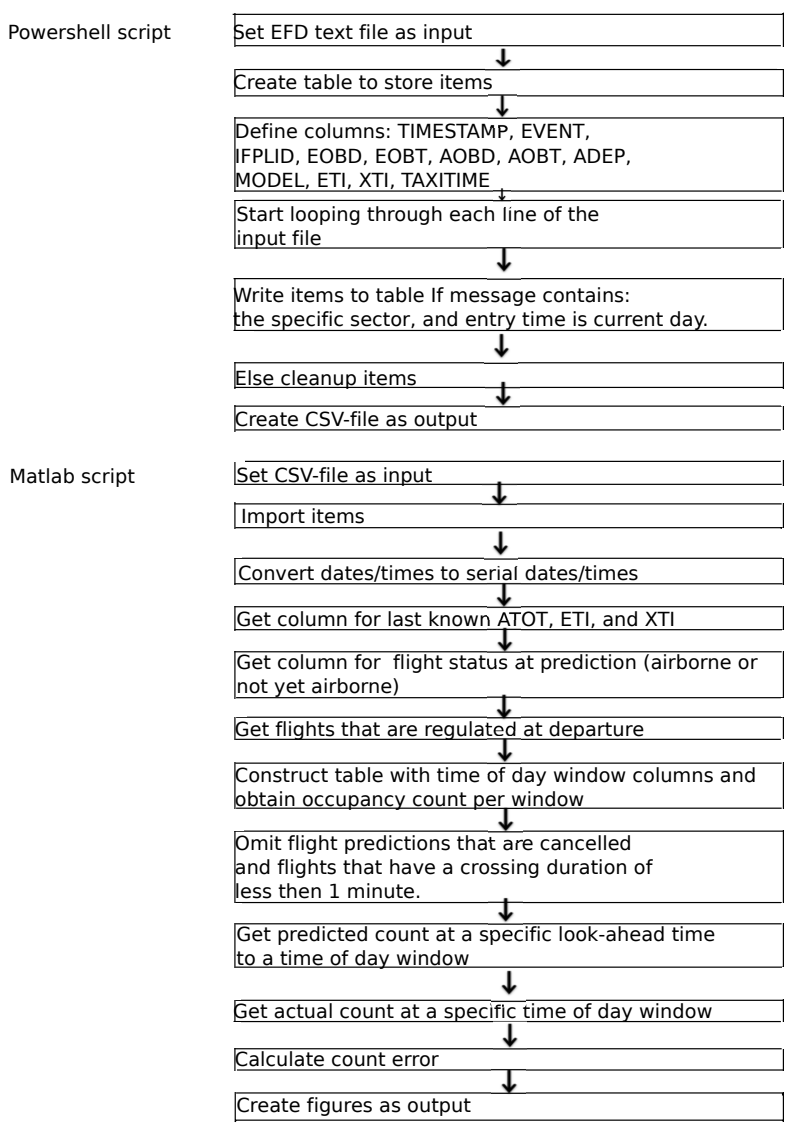

Figure 3.3: Flow diagram to obtain sector occupancy count in section 5.3, from EFD file.

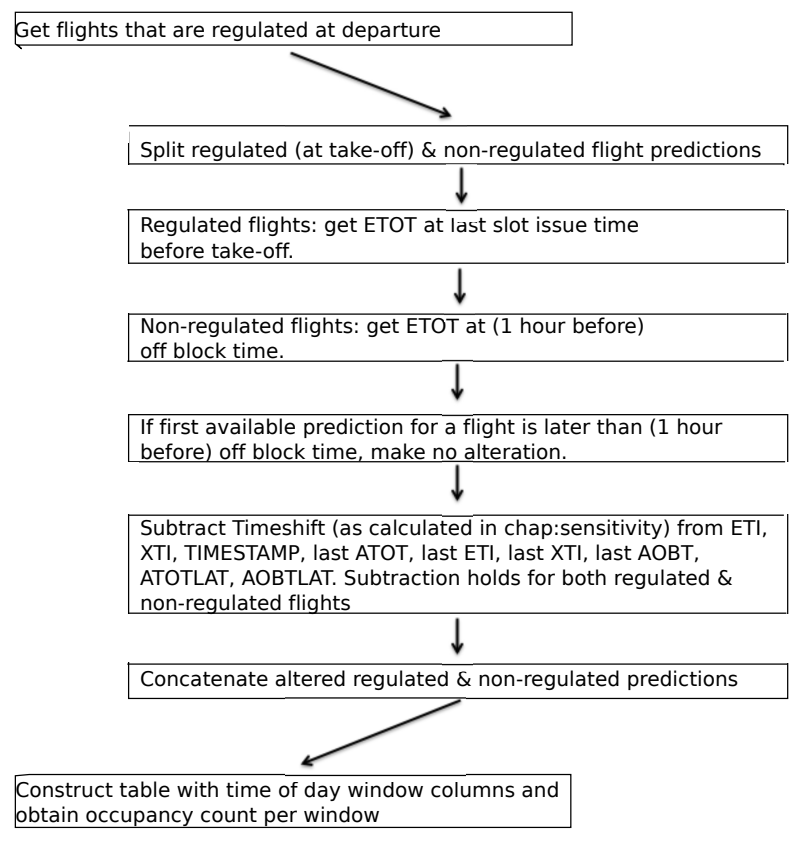

Figure 3.4: Additional part of flow diagram in figure 3.3 to obtain altered sector occupancy count in section 8.3.2.

\subsection{Methodology}

This section gives the used equations for the statistical and sensitivity analysis.

Quantification of uncertainty is mainly done by calculating prediction error as function 
of look-ahead time. Prediction error is given in terms of departure time, arrival time, entry time, entry count, or occupancy count. In order to understand the calculation of prediction error and look-ahead time, two important notions of time used in this thesis are; time of prediction, and entry time prediction. The former is also referred to as timestamp, indicating the time of day at which a prediction message is issued. The latter is defined as a time of day at which the flight is predicted to enter a sector.

Look-ahead time, using the term timestamp described above, is defined as:

$$
\text { look-ahead time }=\text { entry time } \text { actual }- \text { timestamp }_{\text {prediction }}
$$

Further explanation of look-ahead time by an example with an individual flight is given in section 1.4

Prediction error is the difference between the real value and the predicted value. Prediction error for different metrics, based on a look-ahead time, are :

$$
\text { time prediction error }(\epsilon)=\text { entry time }{ }_{\text {actual }}-\text { entry time }_{\text {prediction }}
$$

absolute time prediction error $(\epsilon)=\mid$ entry time $_{\text {actual }}-$ entry time $_{\text {prediction }} \mid$

$$
\text { count prediction error }(\epsilon)=\text { count }_{\text {actual }}-\text { count }_{\text {prediction }}
$$

$$
\text { percentage count prediction error }(\epsilon)=\frac{\text { count }_{\text {actual }}-\text { count }_{\text {prediction }}}{\text { count }_{\text {actual }}} * 100 \%
$$

Furthermore the mean is calculated by:

$$
\bar{x}=\frac{x_{1}+x_{2}+\cdots+x_{n}}{n}
$$

And the standard deviation used is the corrected sample standard deviation:

$$
s=\sqrt{\frac{1}{n-1} \sum_{i=1}^{n}\left(x_{i}-\bar{x}\right)^{2}}
$$

where $\mathrm{n}$ is sample size, and $\mathrm{x}$ are observed values. If data is distributed normally, approximately 68 percent of the observed values are within one standard deviation of the mean (figure 5.2).

A negative time error means the aircraft is earlier than planned while a positive error means that the aircraft is delayed. A negative count error means there is an overprediction i.e. forecast is larger than actual count. Likewise, a positive count error means there is an under-prediction. See figure 3.5. 


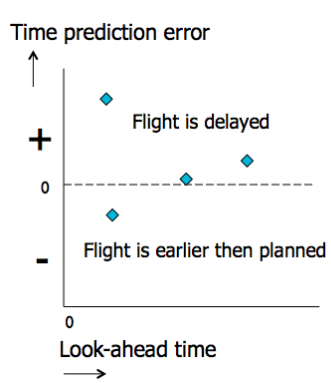

(a) Time error.

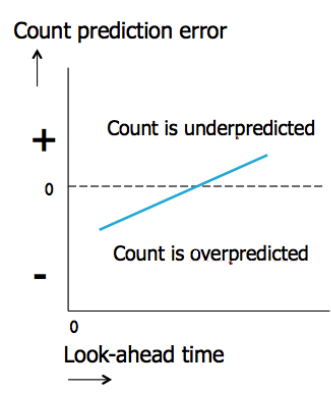

(b) Count error.

Figure 3.5: Example positive and negative prediction error.

\subsection{Confidence Bounds}

Confidence interval is a statistical term for an interval, calculated from observations, that includes the frequency of the regarded mean or another value of interest. The frequency that the confidence interval contains the value of interest is dependent on the confidence level. In applied practice, confidence levels of $95 \%$ are typically taken, therefore this level is chosen in this thesis. A confidence level of $95 \%$ can be explained in terms of samples: "If the process of obtaining a calculated value of interest is repeated several times, the calculated confidence interval would incorporate the true parameter (mean or other value of interest) $95 \%$ of the time".

Assuming the observations are distributed normally, and the mean $(\mu)$ is the (unknown) parameter of interest. The confidence interval for $\mu$ according to [2] :

$$
\left(\bar{x}_{n}-z_{\alpha / 2} \frac{\sigma}{\sqrt{n}}, \bar{x}_{n}+z_{\alpha / 2} \frac{\sigma}{\sqrt{n}}\right) .
$$

where $\bar{x}_{n}$ is the sample mean, $\mathrm{n}$ is the number of observations, $\sigma$ is the standard deviation, and $z_{\alpha / 2}$ is the $\mathrm{z}$-value. The $\mathrm{z}$-value for a standard normal distribution is the value that indicates how many standard deviations away from the mean the observations are. If you want to contain $95 \%$ of the observations, this corresponds to a z-value of $z_{\alpha / 2}=1.96$ [2].

\section{Determining the sample size}

Equation 3.8 can be rewritten to obtain the width of a confidence interval:

$$
2 \cdot z_{\alpha / 2} \frac{\sigma}{\sqrt{n}}
$$

Requiring that $\mathrm{w}$ (confidence interval) should be the following, to find the smallest $\mathrm{n}$ that satisfies:

$$
2 \cdot z_{\alpha / 2} \frac{\sigma}{\sqrt{n}} \leqslant w
$$


or for a given confidence interval the sample size should be:

$$
n \geqslant\left(\frac{2 \cdot z_{\alpha / 2} \sigma}{w}\right)^{2}
$$

\section{Prediction interval}

For the fitted curves in this report, prediction bounds are calculated. Prediction bounds are regularly called confidence bounds because a confidence interval is calculated for the fitted curve. Again a $95 \%$ confidence level is taken. The given prediction interval in figure 5.8 indicate that the probability is $95 \%$ that a new observation is contained within the lower and upper prediction bounds. 


\section{Chapter 4}

\section{Departure Time Predictability}

The departure time predictability has a high influence on the sector demand predictability. This chapter starts with giving an overview of the amount of flights included for the analysis. Then an explanation of the used methodology is given, followed by calculations of the confidence bounds. Section 4.5 discusses distributions for departure time prediction error. Number of flights is plotted as function of departure time prediction error, which is interesting for Airport Managers, to show how many flights depart within the departure slot tolerance window.

\subsection{Population Sample}

For the analysis done in this chapter some flights are excluded. The following itemization shows the exclusions made, where $\mathrm{A} \subset \mathrm{B}$ means that $\mathrm{A}$ is part of the bigger group $\mathrm{B}$.

- Flights crossing through airspace Fox1 (figure 4.1c) $\subset$ Flights being present sometime during the day within the extended ECAC area (figure 4.1b).

- Flights that are scheduled air transport $\subset$ Flights crossing through airspace FOX1.

- Flights that are not cancelled $\subset$ Flights that are scheduled air transport.

- Flights that were predicted to enter and did enter $\subset$ Flights that are not cancelled.

Flights through airspace Fox1 are analyzed because this airspace is very big, so it is possible to obtain a larger number of flights. Approximately half of all daily flights in Europe cross this airspace, however it is not an actual sector. Furthermore military and general aviation flights are excluded because previous research indicates that they hardly adhere to flight plans, resulting in large prediction error [11]. Flights that were predicted to enter, but did not enter, are excluded, because it is unrealistic to calculate a prediction error if the flight never entered the sector. 
To obtain a large population sample, 6 days are analyzed, of which 3 busy days and 3 normal days. The days are 27/06/2013, 28/06/2013, 29/06/2013, 05/03/2014, 06/03/2014, and 07/03/2014. For the period March 2013 - March 2014, the 28th of June 2013 has the largest number of daily flights, and the 7th of March 2014 has approximately the average number of daily flights. This results in a population sample of:

- Non-regulated flights $=49558$

- Regulated flights (at departure) $=7042$

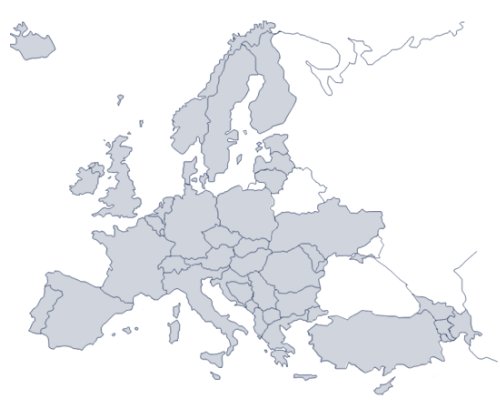

(a) ECAC member states

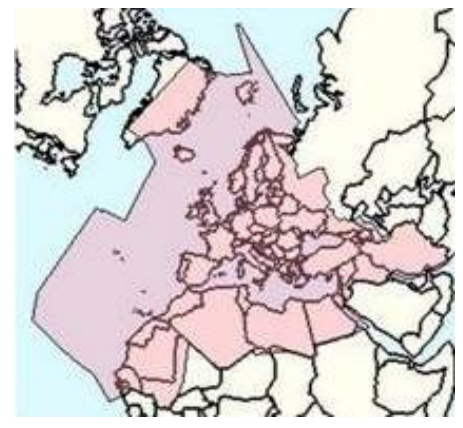

(b) Extended ECAC area

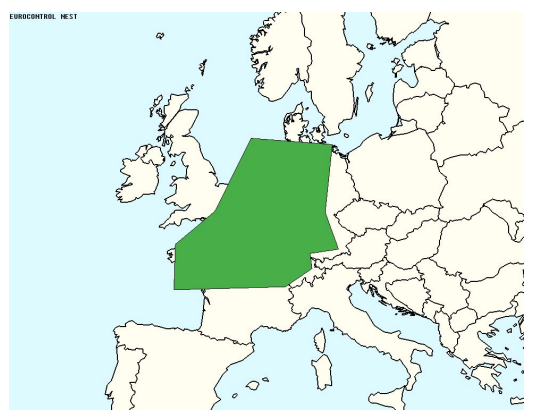

(c) Airspace FOX1 (FL175 and above)

Figure 4.1: Geographical top-down view of the airspaces included in the analysis.

\subsection{Methodology}

In order to calculate departure time prediction error, equation 3.2 is used, where entry time is replaced by departure time. The method used to summarize the prediction error graphically is the kernel density estimate. This is similar to the histogram method, the kernel also constructs a function to represent the probability density, but it is easier with kernel to compare multiple distributions (figure 4.2). The kernel density estimate is give by:

$$
f_{n, h}(x)=\frac{1}{n h} \sum_{i=1}^{n} K\left(\frac{x-x_{i}}{n}\right)
$$


where, $n=$ sample size, and $x$ are the dataset observations [2]. The quality of the kernel density estimate depends on the choice of the Kernel $K$, and the choice of bandwidth $h$. The kernel function is a probability density function typified by $\int_{\infty}^{\infty} K(u) d u=1$ ). For this thesis the bandwidth that is optimal for estimating densities for the normal distribution are chosen. The distributions in section 4.5 can be approximated by a normal distribution; they can be classified as log-normal distributions that are skewed to the left.

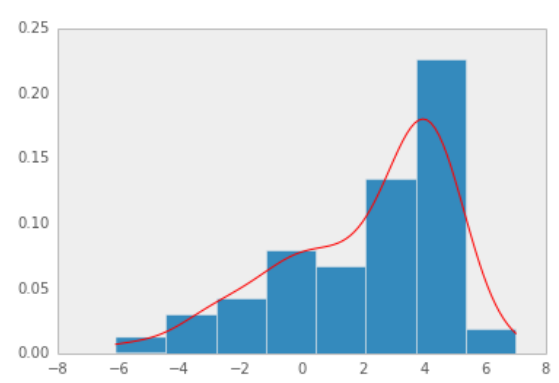

Figure 4.2: Example plot of kernel density estimate and histogram [2].

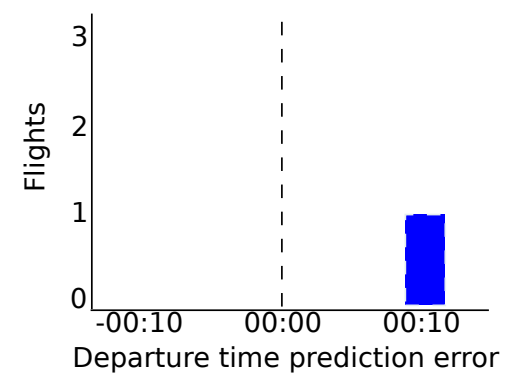

Figure 4.3: Example prediction error for 1 hour look-ahead, for individual flight.

\subsection{Confidence Bounds}

For the figures on prediction error distributions in section 4.5, this section discusses the calculation of confidence bounds for mean departure time prediction error at the initial flight plan (figures 4.8 and 4.9) The sample size is given in section 4.1. Furthermore the mean and standard deviation are given in figures 4.8 and 4.9. For regulated flights the $95 \%$ confidence bounds of the mean departure time prediction error, using equation 3.8 , are :

$$
\left(17: 39-1.96 \frac{31: 15}{\sqrt{6932}}, 17: 39+1.96 \frac{31: 15}{\sqrt{6932}}\right)=(16: 55,18: 23) \quad \text { HH:MM. }
$$

For non-regulated flights, the $95 \%$ confidence bounds of the mean departure time prediction error are:

$$
\left(06: 08-1.96 \frac{14: 07}{\sqrt{49558}}, 06: 08+1.96 \frac{14: 07}{\sqrt{49558}}\right)=(06: 00,06: 16) \quad \text { HH:MM }
$$

\subsection{Individual Flight Example}

Table 1.1 shows that the predicted departure time, 1 hour in advance is 11:20. The actual departure time is 11:30, which yields a positive error of 10 minutes (the flight has been delayed). This is displayed in figure 4.3. Now taking the prediction error for all flights of the population sample in section 4.1, and calculating the kernel density estimates, yields the figures in section 4.5 . 


\subsection{Departure Time Prediction Error}

Figures 4.8 and 4.9 show the departure time \& sector entry time prediction error at the initial flight plan, for respectively non-regulated and regulated flights. From these figures two striking results emerge: sector entry time predictions are better than departure time predictions, and non-regulated flights better adhere to the initial flight plan than regulated flights. The former can be explained due to pilots speeding up / slowing down to adhere to the flight plan. The latter can be explained due to the bigger amount of discrepancy between initially planned and regulated departure times. Turning to figure 4.5, standard deviation of sector entry time prediction error is 4 times better at off block time than at the initial flight plan. Standard deviation of entry time prediction error is 2 times better at take-off than at off block time. Figure 4.7 shows that there is a peak at 5 minutes before the regulated departure time which corresponds to the beginning of the slot $[-5,+10$ min.]. This means that pilots tend to depart as soon as possible because they want to make up for lost time.

Comparing departure time predictability on a set of normal and busy days, it can be seen from appendix B that prediction accuracy is better on normal days. Only departure time \& sector entry error at off block time and during taxi differ with a small value for a normal and busy set of days for regulated flights (see figure B.3 and B.4). This indicates that for small pre-departure look-ahead times, the prediction error is similar irrespective of the traffic level. 


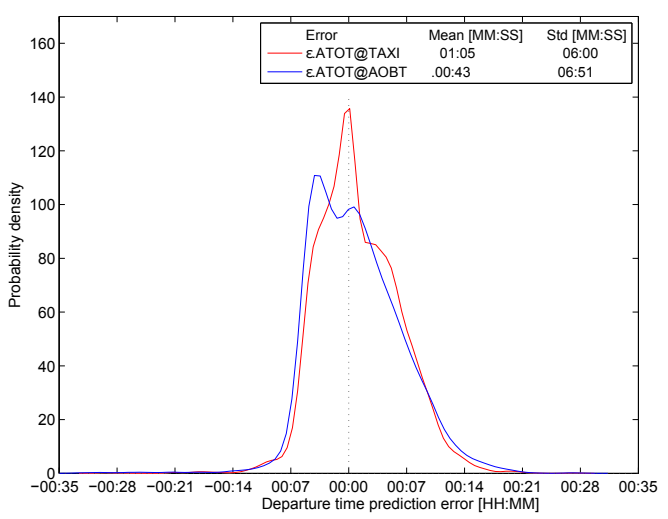

Figure 4.4: Take-off time prediction error during taxi, and at offblock time, for regulated flights

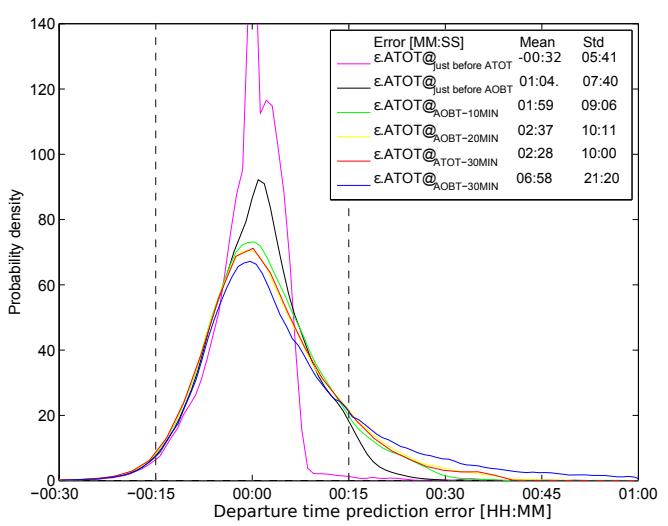

Figure 4.6: Take-off time prediction error at different time instances for non-regulated flights.

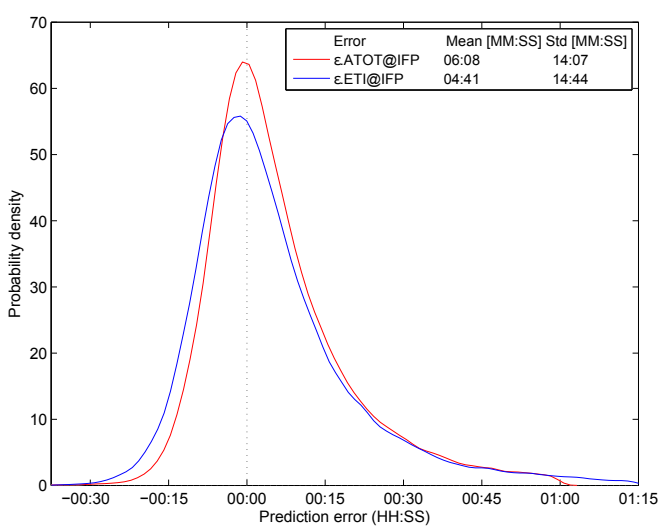

Figure 4.8: Take-off time, and sector entry time prediction error at initial flight plan, nonregulated flights.

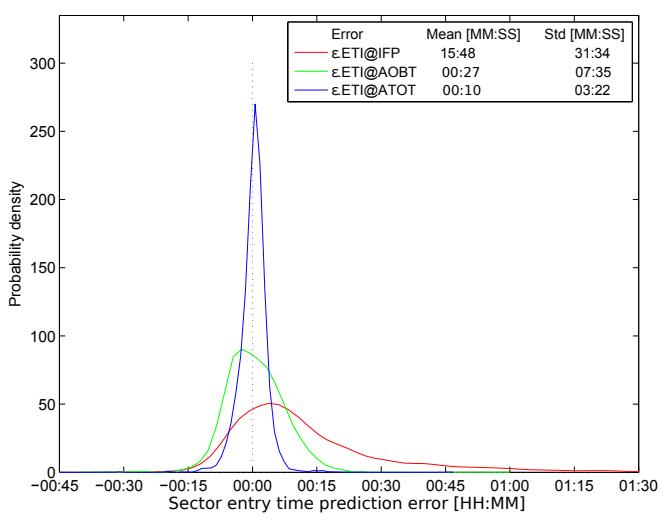

Figure 4.5: Sector entry time prediction error at initial flight plan, at off-block time, and at takeoff, for regulated flights

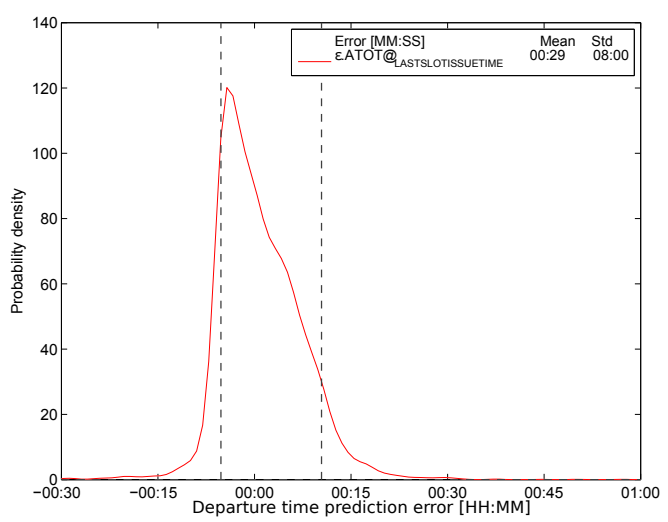

Figure 4.7: Take-off time prediction error at last slot issue time for regulated flights

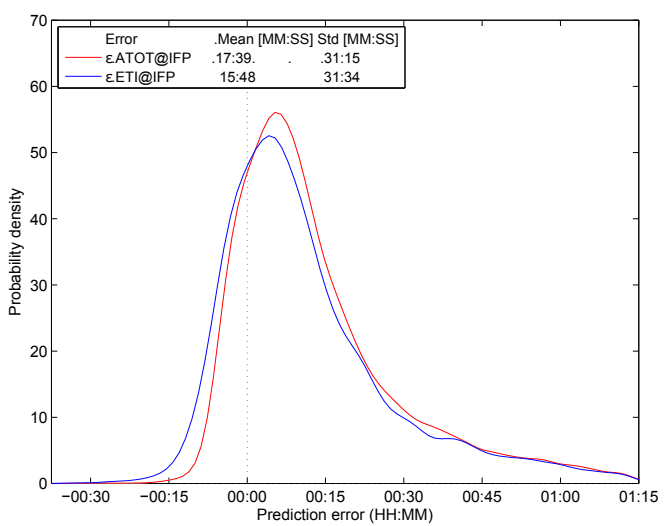

Figure 4.9: Take-off time, and sector entry time prediction error at initial flight plan, regulated flights. 


\subsection{Standard Deviation Prediction Error per Airport}

Turning now from distributions of prediction error to distributions of standard deviation prediction error per airport. To explain this concept, first all prediction errors for the flights in the population sample were calculated. For regulated flights, the prediction error was calculated at last slot issue time before take-off. For non-regulated flights, prediction error was calculated at 30 minutes before take-off. The next step is to filter the prediction errors for all flights per individual airport. Then the standard deviation of the prediction errors per airport is calculated (average standard deviation per airport). This results in figure 4.10. Most airports have a standard deviation of departure time prediction error of 9 minutes (non-regulated flights). Standard deviation of 9 minutes means that $68 \%$ of all flights depart not more than 9 minutes earlier, or later than the mean departure time prediction error which is $\sim 2.5$ minutes (figure 4.6 ). This is good because the departure time tolerance window is \pm 15 minutes, and \pm 9 minutes falls within this window. The slot tolerance window for regulated flights is $[-5,+10]$ minutes. Take note that the error distribution is dependent on the prediction look-ahead time.

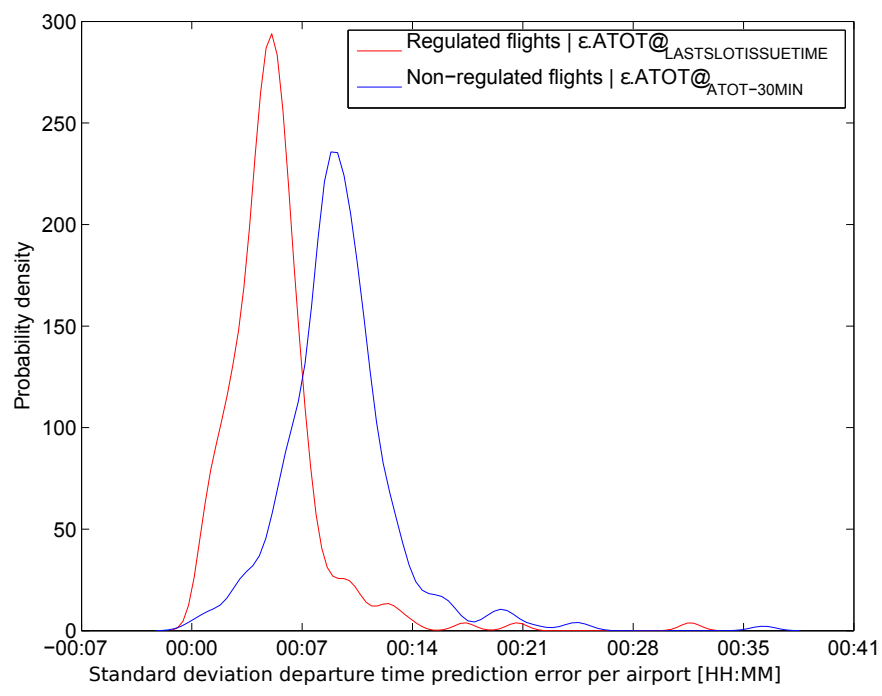

Figure 4.10: Standard deviation of the departure time prediction error per airport. Error for regulated flights at last slot issue time, error for non-regulated flights at 30 minutes before take-off.

\subsection{Conclusions}

This section gives an overview of the main results from this chapter:

- Sector entry time predictions are better than departure time predictions. This is because pilots speed up/slow to recover the delay at departure and adhere to the planned entry times.

- Non-regulated flights better adhere to the flight plan than regulated flights. This is 
because the initial filed flight plan does not take into account regulated departure times.

- Regulated flights tend to depart at the beginning of the departure slot tolerance window to recover lost time on the ground.

- At 30 minutes before off block time, most airports have an average standard deviation departure time prediction error of 9 minutes. This is good because it falls within the departure time tolerance window of \pm 15 minutes. 


\section{Chapter 5}

\section{Sector Demand Predictability}

This chapter consist of three parts; sector entry time, sector occupancy count, and sector entry count. Above metrics are plotted as function of look-ahead time to sector entry. Aim is to quantify the current predictability in a manner that is relatable to the Flow Manager's perspective.

\subsection{Population Sample}

Table 5.1: Characteristics of sectors that are analyzed. Note that the day is $28 / 06 / 2013$ unless stated otherwise.

\begin{tabular}{llllllll}
\hline Sector & $\begin{array}{l}\text { Flight } \\
\text { level }\end{array}$ & $\begin{array}{l}\text { Flights } \\
\text { entered }\end{array}$ & $\begin{array}{l}\text { Flights } \\
\text { regulated } \\
\text { at } \\
\text { departure }\end{array}$ & $\begin{array}{l}\text { Flights } \\
\text { cancelled }\end{array}$ & $\begin{array}{l}\text { Climbing } \\
\text { traffic } \\
{[\%][3]}\end{array}$ & $\begin{array}{l}\text { Cruising } \\
\text { traffic } \\
{[\%][3]}\end{array}$ & $\begin{array}{l}\text { Descending } \\
\text { traffic } \\
{[\%][3]}\end{array}$ \\
\hline Jever Low & $245-335$ & 449 & 52 & 14 & 35 & 25 & 43 \\
Koksy High & $335+$ & 701 & 121 & 34 & 41 & 35 & 27 \\
Koksy Low & $245-335$ & 832 & 138 & 51 & 48 & 12 & 43 \\
$\begin{array}{l}\text { Koksy Low } \\
\text { 29-06-13 }\end{array}$ & $245-335$ & 742 & 184 & 39 & 53 & 9 & 43 \\
$\begin{array}{l}\text { Koksy Low } \\
\text { 05-03-14 }\end{array}$ & $245-335$ & 616 & 22 & 27 & 42 & 11 & 48 \\
$\begin{array}{l}\text { Koksy Low } \\
\text { 07-03-14 }\end{array}$ & $245-335$ & 758 & 75 & 33 & 43 & 11 & 48 \\
Lux Low & $245-335$ & 769 & 155 & 42 & 38 & 13 & 51 \\
Nicky High & $335+$ & 826 & 186 & 40 & 28 & 57 & 17 \\
$\begin{array}{l}\text { Bordeaux } \\
\text { X4 }\end{array}$ & $365+$ & 495 & 113 & 13 & 9 & 87 & 3 \\
\hline
\end{tabular}




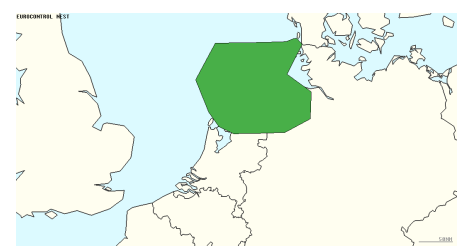

(a) Jever Low

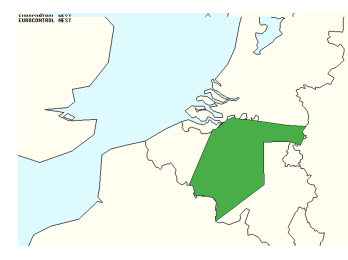

(d) Nicky High

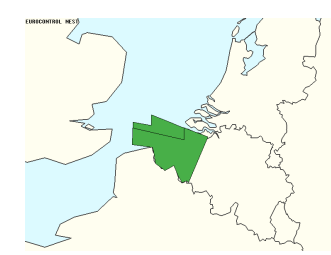

(b) Koksy High

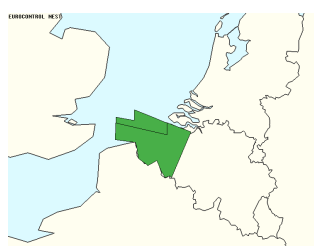

(e) Koksy Low

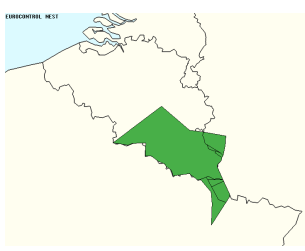

(c) Lux Low

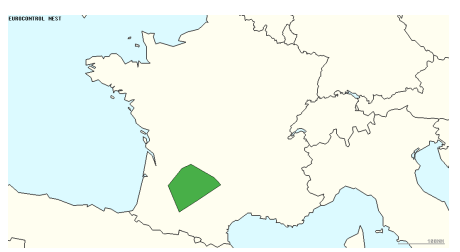

(f) Bordeaux X4

Figure 5.1: Geographical top-down view of analyzed sectors [3].

Table 5.1 describes characteristics of the sectors that are analyzed in this chapter. Figure 5.1 shows top-down views of the regarded sectors. These are all Maastricht Upper Area Control centers managed by Eurocontrol except the Bordeaux sector, so expertise from Air Traffic Controllers and Flow Managers is readily available. In terms of traffic volume, it is the second busiest air traffic control centre in Europe, after London. [4] states that sectors Koksy Low, and Lux Low are classified as high complexity sectors. Low complexity sectors are Jever Low and Bordeaux. Sector Koksy, Nicky, and Lux have a lot of departing traffic from London, Paris, Frankfurt, and Amsterdam. Departure sectors involve a much greater proportion of pre-departure flights than other sector types. Because departure time predictions are highly uncertain, predictions for departure sectors are more uncertain [12].

Four days were analyzed, the 28th and 29th of June 2013 and the 5th and 7th of March 2014. The 28th of June 2013 is chosen because this day is the day with the largest number of flights in 2013 in Europe. 5th and 7th of March are the days where the daily traffic is approximately the same number as the average daily traffic for the time period March 2013 - March 2014. Note that the analysis in this chapter is done for the 28th of June 2013, unless stated otherwise.

On the 28th of June 2013, several disruptions occurred. There were high delays due to capacity at some Greece airports. There was high demand at Cannes airport. Also London Heathrow and Zurich airport were regulated due to capacity. Manchester airport was regulated due to a new tower. Geneva airport had staffing issues. Schiphol airport arrival and departure were regulated due to radio frequency problems. Several ATC control centers were regulated due to capacity in France and UK. 
Table 5.2: Goodness of fit values for prediction error vs. look-ahead curve fit. Sector Koksy High

\begin{tabular}{llll}
\hline Degree & $R^{2}$ & SSE & RMSE \\
\hline 1 & 0.9883 & 0.0321 & 0.0012 \\
2 & 0.9813 & 0.0512 & 0.0016 \\
3 & 0.9780 & 0.06 & 0.0017 \\
4 & 0.9886 & 0.031 & 0.0012 \\
5 & 0.9927 & 0.0177 & $9.198^{*} 10^{-4}$ \\
6 & 0.9922 & 0.0214 & 0.001 \\
7 & 0.9900 & 0.0273 & 0.0011 \\
8 & 0.9879 & 0.0330 & 0.0013 \\
9 & 0.9917 & 0.02 & $9.76^{*} 10^{-4}$ \\
\hline
\end{tabular}

Table 5.3: Goodness of fit values for prediction error vs. look-ahead curve fit. Sector Lux Low

\begin{tabular}{llll}
\hline Degree & $R^{2}$ & SSE & RMSE \\
\hline 1 & 0.9802 & 0.0505 & 0.0015 \\
2 & 0.9783 & 0.0554 & 0.0016 \\
3 & 0.9797 & 0.0516 & 0.0016 \\
4 & 0.9812 & 0.0478 & 0.0015 \\
5 & 0.9774 & 0.0576 & 0.0016 \\
6 & 0.9787 & 0.0544 & 0.0016 \\
7 & 0.9928 & 0.0182 & $9.28^{*} 10^{-4}$ \\
8 & 0.9853 & 0.0373 & 0.0013 \\
9 & 0.9871 & 0.0329 & 0.0012 \\
\hline
\end{tabular}

\subsection{Sector Entry Time Predictability}

\subsubsection{Methodology}

The methodology used to obtain prediction error and look-ahead time is give in section 3.3. Henceforth plotted prediction errors versus look-ahead are called data points. This section elaborates on how a curve is fitted through these data points, and then discusses outlier removal. The curve fit through the data points is also called mean sector entry time prediction error.

Curve fitting is the process of constructing a mathematical function, that fits best to the data points. In this section, a fitted curve aids to show the relationship between prediction error and look-ahead time. One way to determine the goodness of fit is by looking at the coefficient of determination or the $R^{2}$, a number to indicate how well the data fits to the mathematical function. A $R^{2}$ of 1 indicates that the curve perfectly fits the data. The function that fits the data points best is the polynomial function, i.e. a $n$th degree polynomial. The degree which has been found to consistently show best $R^{2}$ values is the 9 th degree. Table 5.2 and 5.3 shows the obtained $R^{2}$ values for different degrees of polynomial functions. Although the $R^{2}$ for the 9 th degree is not the degree where the $R^{2}$ is closest to 1 , it is consistently the second or third closest to 1 . Other used goodness of fit statistics are SSE (sum of square errors) and RMSE (random mean squared error). SSE is a measure of the discrepancy between the data points and the curve fit. A value closer to 0 indicates less discrepancy. RMSE is an estimate of the standard deviation of the random component of the data points. A value closer to 0 indicates less standard deviation.

Curve fits of data points by a (9th degree) polynomial fit could be overfitted at large look-ahead times (the sudden swing at 6 hour look-ahead in figure 5.9). Overfitting is a modeling flaw were the curve fit is an overly complex model to explain idiosyncrasies in the data points. Although the $R^{2}$ is high, the model attempts to conform too closely to the smaller amount of data points at longer look-ahead times.

An outlier is defined as a data point that is distant from other data points. For example a flight from La Guardia, New York predicted to enter sector Koksy High at approximately 08:00 but was delayed for 13 hours and entered the sector at 21:00 in the evening. This 


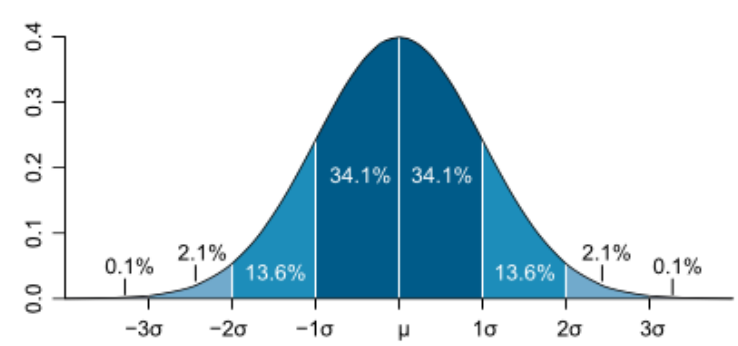

Figure 5.2: Example normal distribution plot. [2].

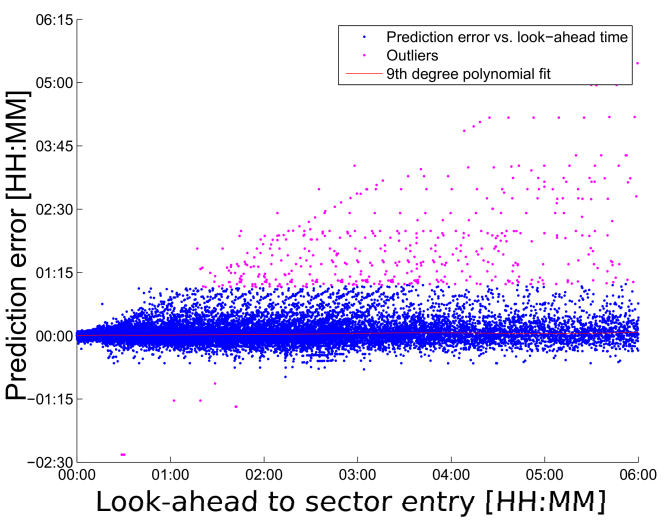

Figure 5.3: Prediction error outliers, for sector Koksy High.

observation can be seen northeast in figure 5.7a. The prediction error is assumed to be normally distributed as shown in section 4.5 . Therefore about $99.7 \%$ of values drawn from a normal distribution are within three standard deviation away from the mean (figure 5.2). It is chosen to exclude data points which are further away than three standard deviations from the mean (pink dots in figure 5.3). Therefore the mean sector entry time prediction error represents a majority of the data.

\subsubsection{Individual Flight Example}

Prediction error versus look-ahead for the British Airways flight is plotted in figure 5.4. It can be seen that the polynomial curve fit is a good representation of the variation of the data points, as the $R^{2}$ is 0.9935 .

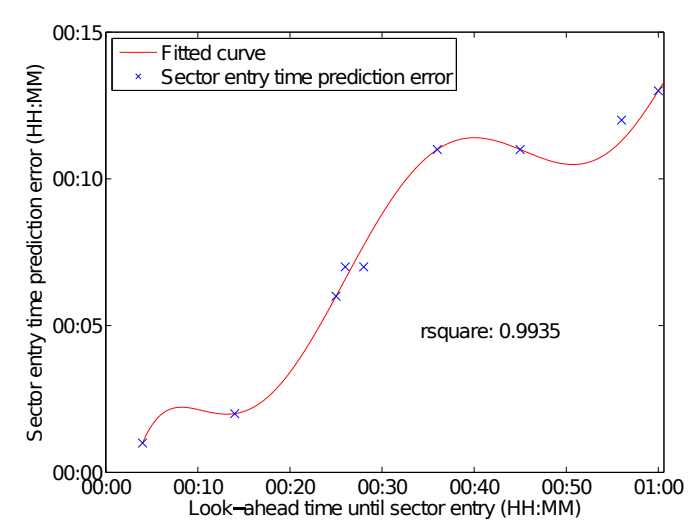

Figure 5.4: Example of curve fit through prediction error vs. look-ahead, for individual flight.

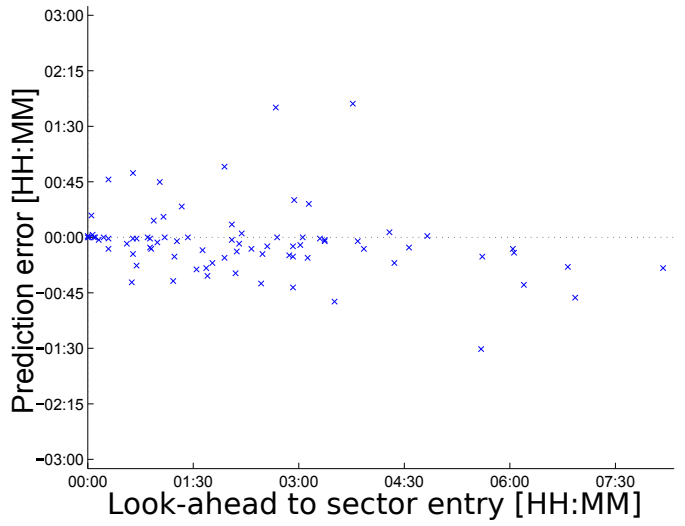

Figure 5.5: Sector entry time prediction error, last available predictions before entry. Flights from London Heathrow. Sector Koksy High. 


\subsubsection{Individual Departure Airport}

This section discusses prediction error versus look-ahead data points for all flights departing from a specified airport that entered sector Koksy High, on the 28th of June 2013. Figure 5.5 shows last available prediction error per flight, just before entry. These predictions are made while the flights were airborne, it can be seen that more flights were earlier than planned (more negative prediction error data points). From the last available prediction before entry, now turning to all predictions before entry, but excluding predictions before off block time, this yields figure 5.6a. Blue dots are predictions that were made while the flight was airborne, green are predictions while the flight was taxiing. Prediction error from flights through sector Koksy High, departing from London Heathrow, Paris Charles de Gaulle and Frankfurt am Main are compared in figure 5.6. From this figure we can see that prediction error is more accurate, and less dispersed if the flight time to the sector is longer. Longer flight times allow for the pilot to speed up or slow down to adhere to the predicted entry time in the flight plan.

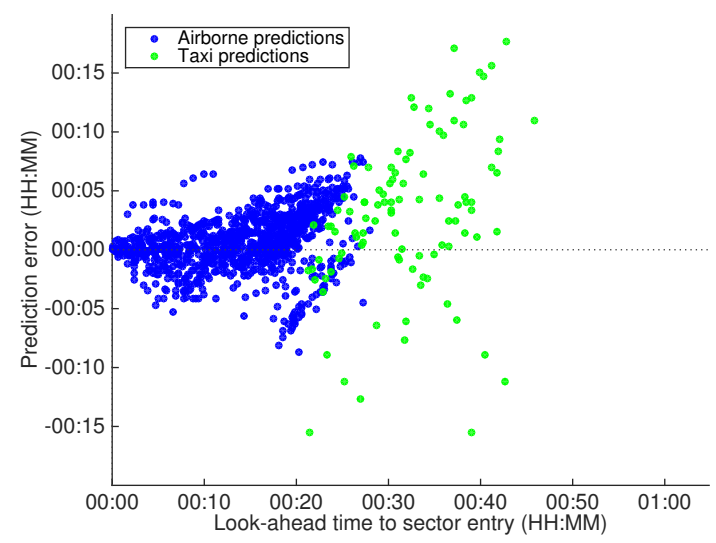

(a) London Heathrow (flights $=97$, observations $=1137)$

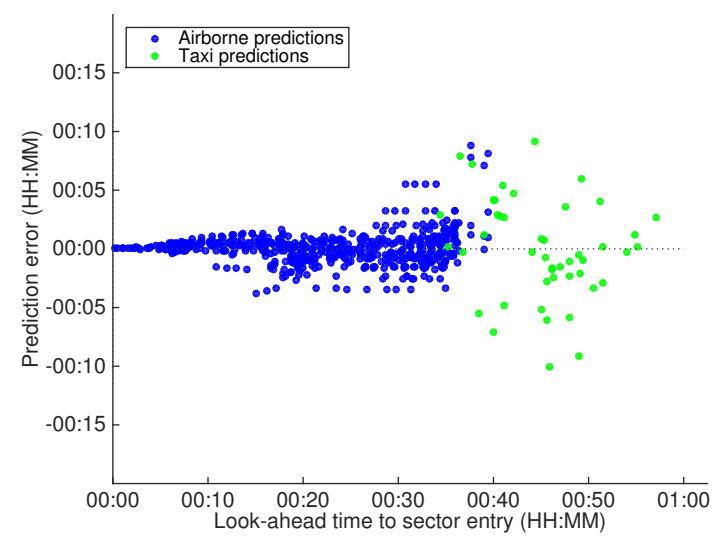

(b) Frankfurt am Main (flights $=36$, observations $=637$ )

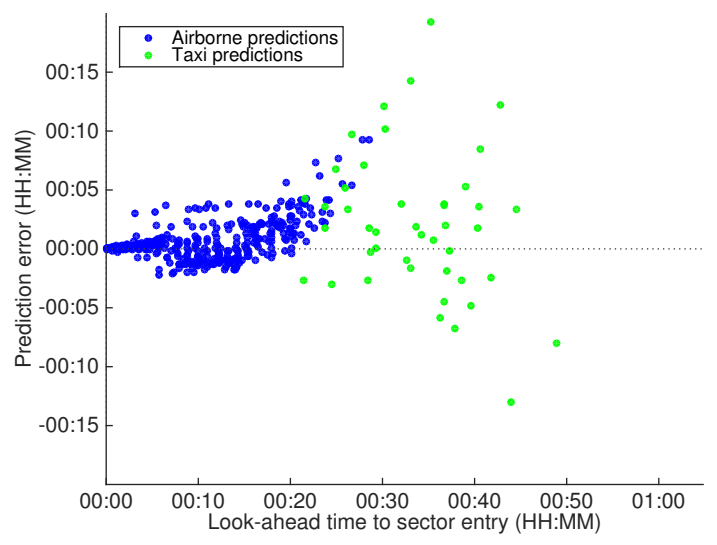

(c) Paris Charles de Gaulle (flights $=29$, observations $=389)$ )

Figure 5.6: Sector entry time prediction error, for flights from different origin airports. Sector Koksy High. 


\subsubsection{Individual Sector}

Now turning from prediction error of flights from an individual airport, to flights from all airports going through sector Koksy High, represented in figure 5.7a. More data points are positive, which means that flights tend to be delayed than earlier. Red dots indicate predictions that were made while the flights have not yet departed. Zooming in on 6 hours look-ahead yields figure $5.7 \mathrm{~b}$, in the south of the figure there are rows of blue dots. A single row are predictions for the same flight. Note that prediction error in this thesis is quantified from 0 to 6 hours look-ahead, at 6 hours look-ahead most flight plans have been filed (section 5.2.6), and on the day of operations the flow managers does not look further than this time period.

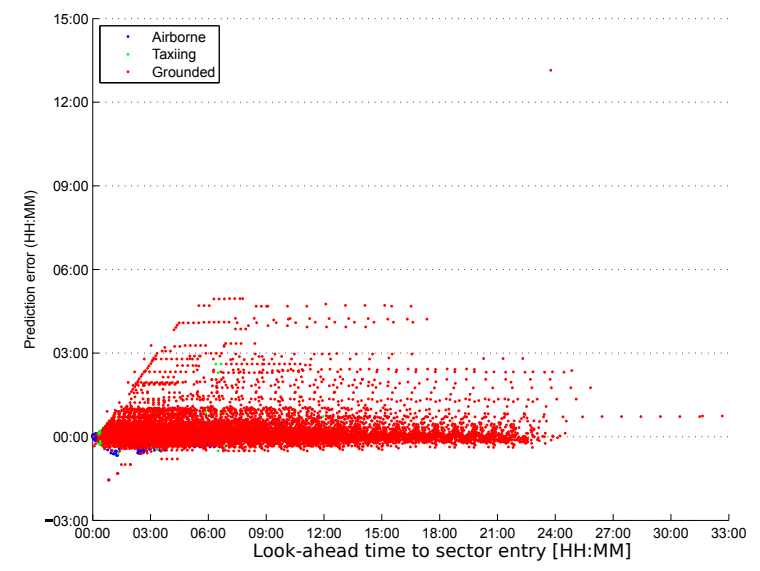

(a) Look-ahead of 33 hours (observations: 27172).

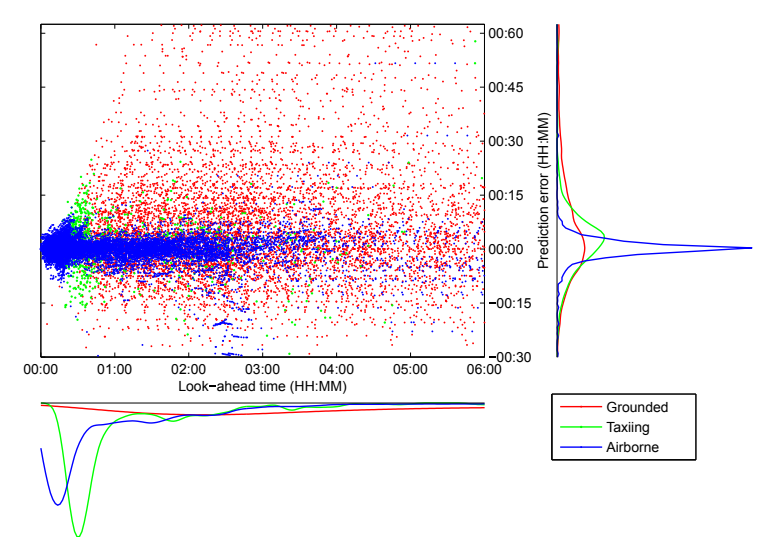

(b) Look-ahead of 6 hours (zoom in), with marginal kernel density estimates

Figure 5.7: Sector entry time prediction error, for all flights on 28th of June 2013. Sector Koksy High.

A curve is fitted (figure 5.8a) through the data points in figure 5.7. Methodology of curve fitting and outlier exclusion is explained in section 5.2.1, the curve represents the mean sector entry time prediction error for Koksy High. Hereby predictions bounds are calculated, indicating that the probability is $95 \%$ that a new observation is contained within these bounds. Taking only absolute prediction error values to show variation of prediction error, this yields figure $5.8 \mathrm{~b}$. Inferences on relative sector performance are made in the next section, where multiple sector are compared.

\subsubsection{Multiple Sectors}

Turning from mean sector entry time prediction error for a single sector, now to multiple sectors. It can be seen from figure 5.9a, that flights on a busy day have a higher probability to be delayed than to be earlier, for 2 hours look-ahead and longer. As expected, flights on average tend to fly on time on a normal day, while flights are delayed on a busy day. To show the variation of the error, figure $5.9 \mathrm{~b}$ shows absolute prediction error. It is apparent that the error increases linearly from 0 to 3 hours look-ahead, and then stabilizes around 7 minutes, which is considers as good. 


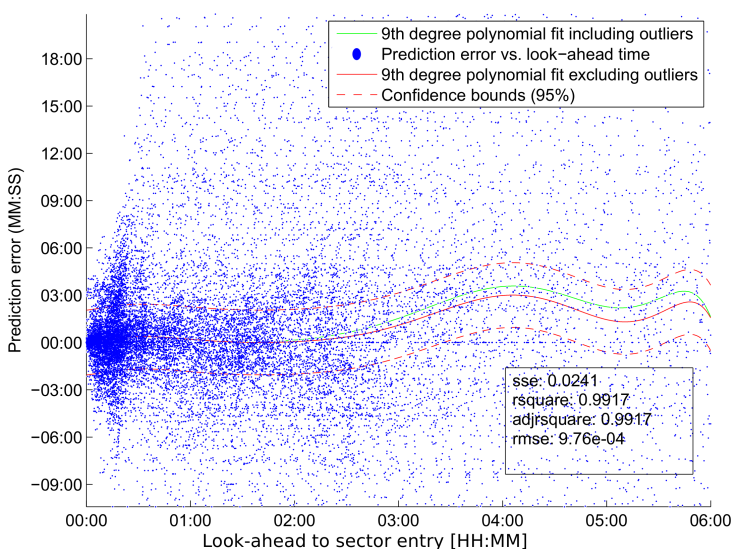

(a) Prediction error vs. look-ahead.

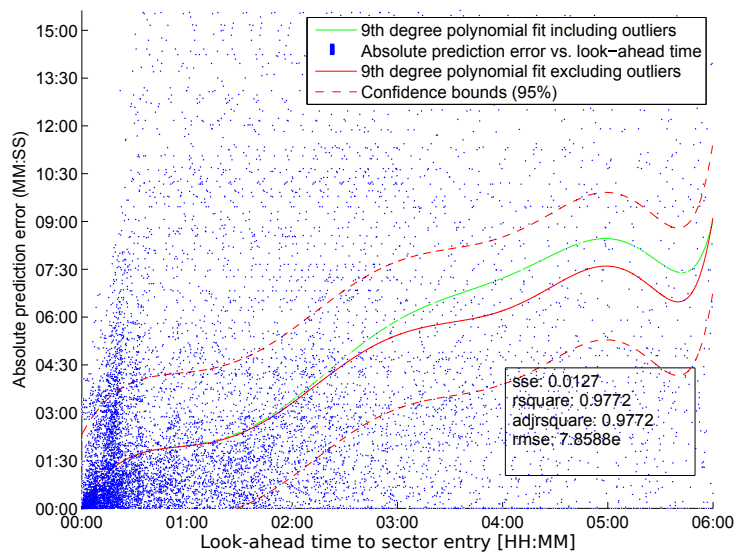

(b) Absolute prediction error vs. look-ahead.

Figure 5.8: Mean sector entry time prediction error for all flights on 28th of June 2013. Sector Koksy High

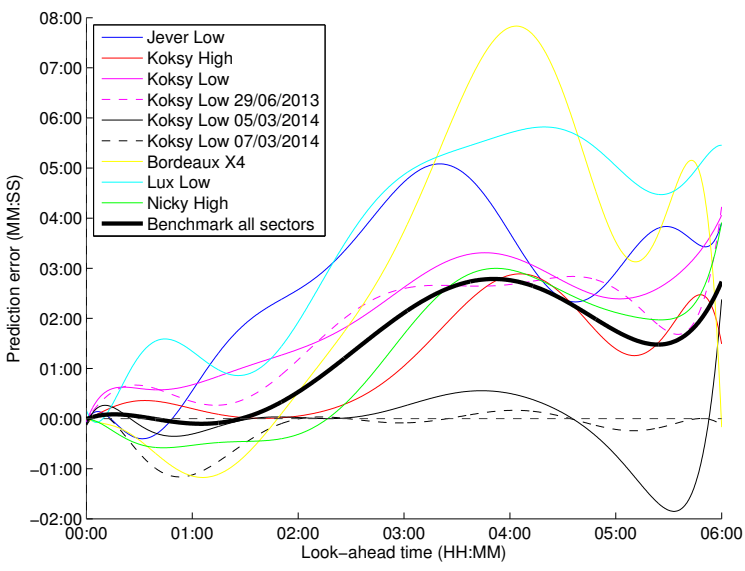

(a) Non-absolute prediction error curve fit.

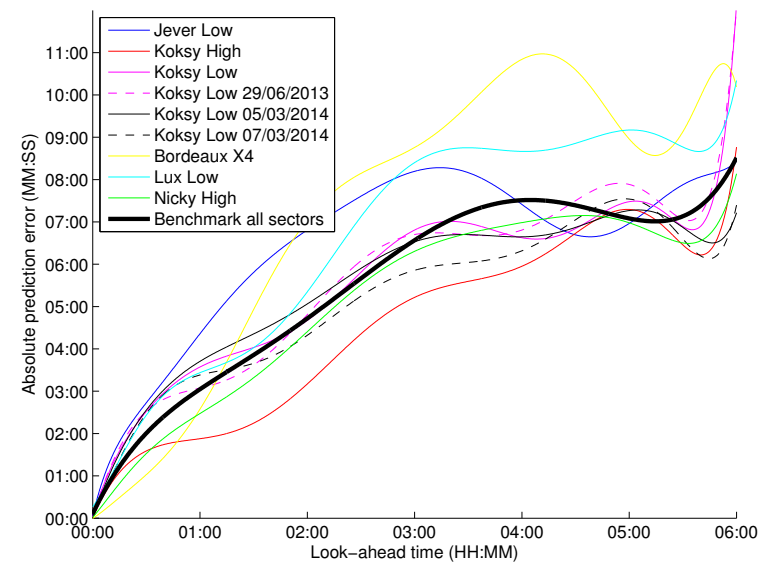

(b) Absolute prediction error curve fit

Figure 5.9: Mean sector entry time prediction error for multiple sectors

Making the transition from average prediction error to standard deviation prediction error. It is apparent from figure 5.10 that the relationship between standard deviation and look-ahead is almost linear. Later then 3 hour look-ahead, the curve becomes volatile because there is a lack of flight plans available. Flight plan availability is quantified in the next section.

\subsubsection{Accumulated Flight Plans}

Figure 5.11 shows how much flight plans have been filed as function of look-ahead times between 0 to 24 hours before sector entry. What is interesting in figure 5.11a is that for some sectors, percentage of accumulated flight plans is not $100 \%$ at sector entry time (i.e. look-ahead $=0$ ). This is because some flights were not predicted to enter, but changed 


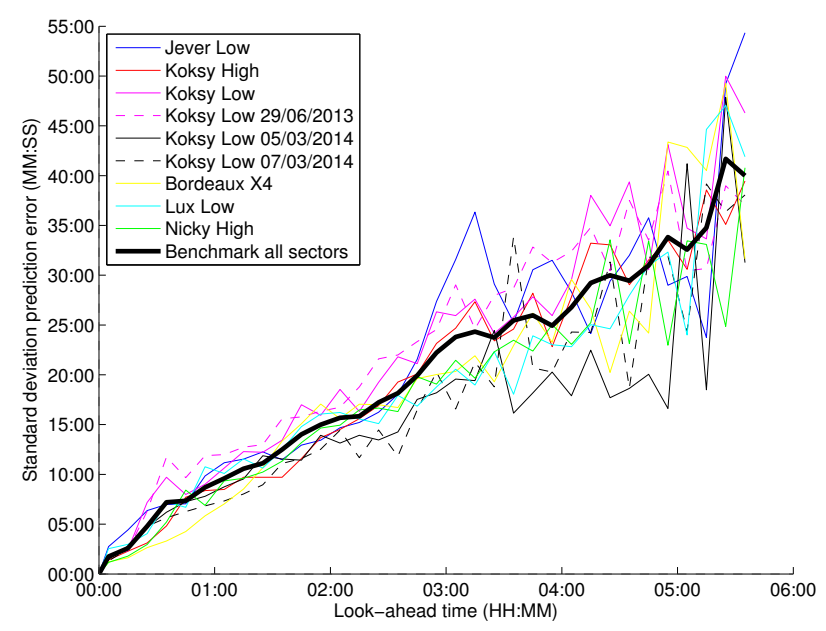

Figure 5.10: Standard deviation sector entry time predication error for multiple sectors.

trajectory at the last moment, and did enter. This concept is elaborated into more detail in section 5.3.9. Furthermore in figure 5.11a some sectors show that more than $100 \%$ of the flight plans have been accumulated at 4 hour look-ahead time. In this case, there were more flights predicted to enter than have actually entered. These 'additional' flights are cancelled or changed trajectory to fly around the sector.

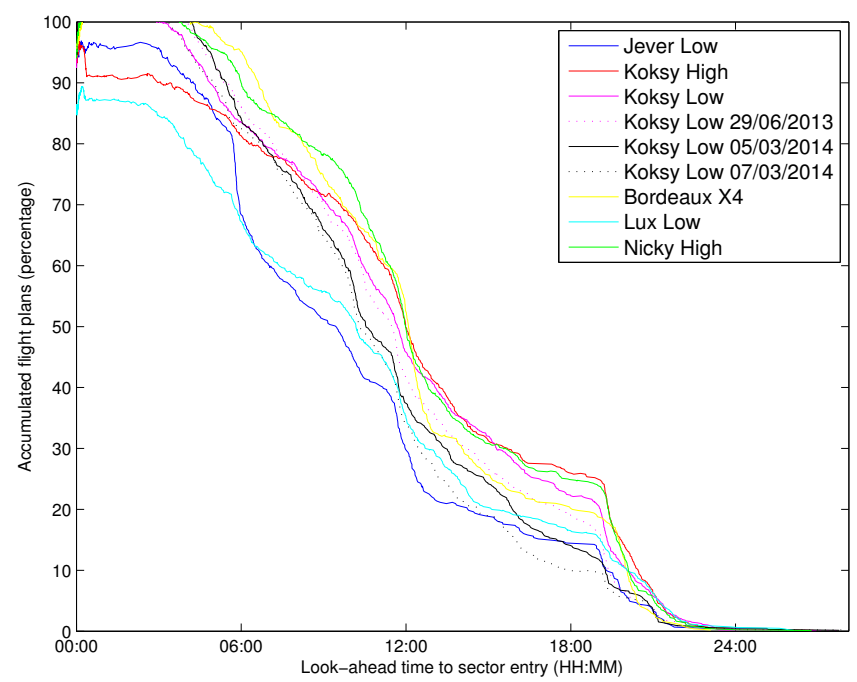

(a) As percentage of flight that actually entered.

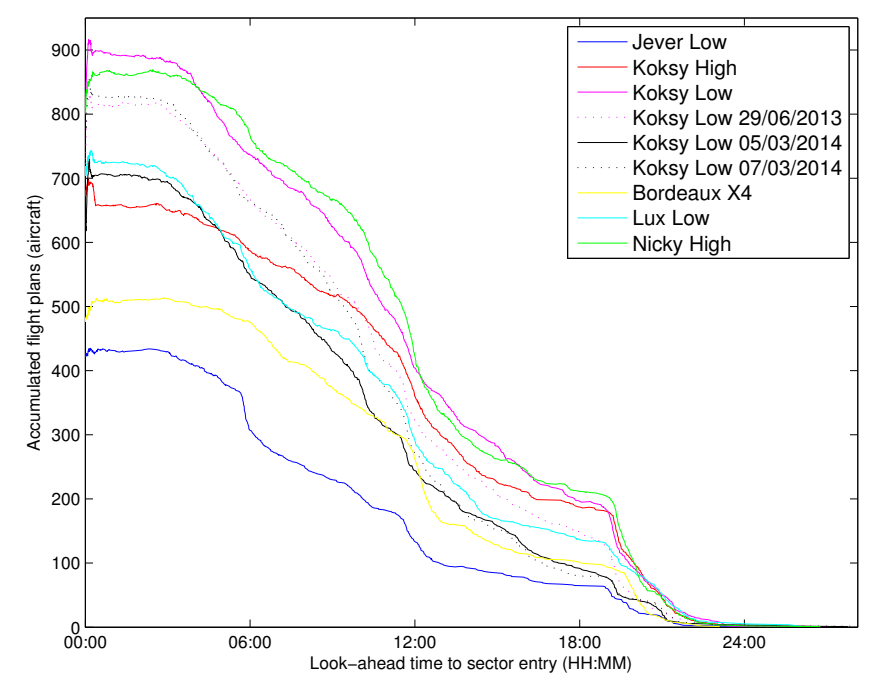

(b) Number of flights

Figure 5.11: Accumulated flight plans for multiple sectors. 


\subsection{Sector Occupancy Count Predictability}

This section starts with the definition of occupancy count, then used methodology is explained by an individual flight example, followed by assumptions made. Thereafter predicted \& actual count is quantified in different ways. First by mean \& standard deviation of predicted count error as function of look-ahead time. Secondly by comparing actual \& predicted count as function of time of day. Then count volatility is discussed by looking at flights deviating from the predicted trajectory.

\subsubsection{Definition}

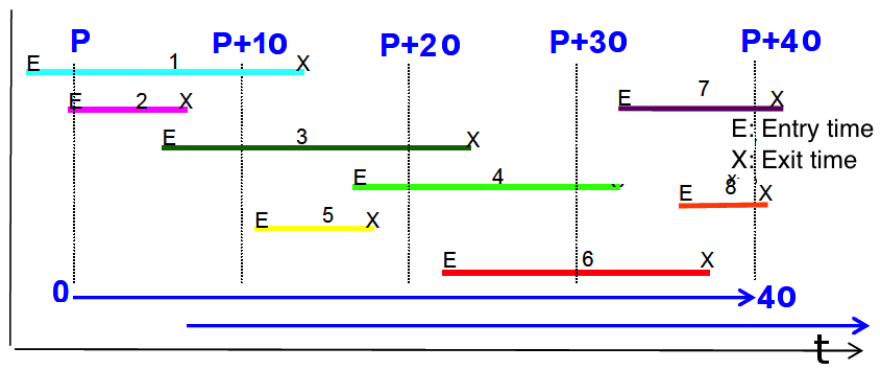

Figure 5.12: Example occupancy count. Skip time $=10$ minutes and window $=10$ minutes.

Occupancy count for a given sector is defined as the number of flights inside the sector during a selected time period. The time period, also called window, of 10 minutes is chosen in this thesis because this is the conventional used period for the Flow Manager. Also software tool NEST uses this window, and therefore can be used for validation purposes.

In figure 5.12 flights are indicated with a color and number. The window (10 minutes) defines the time difference between start and end time of each occupancy counting period. The skip time, indicates the time difference between the start times of two consecutive occupancy counting periods. In this thesis skip time of 10 minutes is chosen because this is also the conventional value.

In figure 5.12 at window $\mathrm{P}$, or between $\mathrm{P}$ until $\mathrm{P}+10$, there are 3 flights: 1,2 , and 3 . At $\mathrm{P}+10$ until $\mathrm{P}+20$, there are 4 flights: $1,3,4$, and 5 . At $\mathrm{P}+20$ until $\mathrm{P}+30$, there are 3 flights, 3 , 4, and 6 . Finally at $\mathrm{P}+30$ until $\mathrm{P}+40$, there are 4 flights: 4, 6, 7, and 8 .

\subsubsection{Confidence Bounds}

Confidence intervals as explained in section 3.4, for mean sector occupancy count prediction error (section 5.3.6), for a look-ahead of 10 and 120 minutes, are calculated. Sector Koksy Low on 28th of June is chosen because it is close to the benchmark curve (figure 5.20) A 10 minute look-ahead is chosen because this is the last prediction before sector entry. 120 minute look-ahead is chosen because at this time the standard deviation benchmark line is fairly stable. For a single day, single sector and time of day between 8 a.m. and 8 p.m., there are 72 windows of 10 minutes. This means the number of observations is 72 . Furthermore the mean and standard deviation for the 2 look-ahead times 
can be obtained from figure 5.19a and 5.19b. The $95 \%$ confidence bounds for 10 minute look-ahead are:

$$
\left(-0.8-1.96 \frac{1.9}{\sqrt{72}},-0.8+1.96 \frac{1.9}{\sqrt{72}}\right)=(-1.23,-0.36) \quad \text { aircraft. }
$$

and for 120 minutes look-ahead:

$$
\left(-1-1.96 \frac{3.8}{\sqrt{72}},-1+1.96 \frac{3.8}{\sqrt{72}}\right)=(-1.88,-0.12) \quad \text { aircraft }
$$

Now turning to percentage prediction error, for a look-ahead time of 10 minutes:

$$
\left(-19.8-1.96 \frac{28}{\sqrt{72}},-19.8+1.96 \frac{28}{\sqrt{72}}\right)=(-26.3,-13.3) \quad \text { percent. }
$$

and for 120 minutes look-ahead:

$$
\left(-12.5-1.96 \frac{33}{\sqrt{72}},-12.5+1.96 \frac{33}{\sqrt{72}}\right)=(-20.1,-4.9) \quad \text { percent. }
$$

To obtain a sample size where the confidence interval is 0.05 aircraft, assuming the standard deviation remains 2.5 aircraft, the sample size should be:

$$
n \geqslant\left(\frac{2 \cdot 1.96 \cdot 2.5}{0.05}\right)^{2}=38416 \text { observations }
$$

This means that 534 days have to be analyzed to obtain this large amount of observations. Take note that sector configurations and traffic levels change day by day, so the daily confidence interval could differ from the confidence interval of 534 days. To obtain a higher number of observations we look at sector Koksy Low for 4 days; 28/06/2014, $29 / 06 / 2014,05 / 03 / 2014$, and $07 / 03 / 2014$.

The $95 \%$ confidence interval for a look-ahead time of 10 minutes:

$$
\left(-0.9-1.96 \frac{1.95}{\sqrt{292}},-0.9+1.96 \frac{1.95}{\sqrt{292}}\right)=(-1.12,-0.68) \quad \text { aircraft }
$$

and look-ahead time of 120 minutes:

$$
\left(-1.3-1.96 \frac{3.67}{\sqrt{292}},-1.3+1.96 \frac{3.67}{\sqrt{292}}\right)=(-1.72,-0.88) \quad \text { aircraft. }
$$

Because the upper confidence bounds calculated in this section are negative, it can be concluded that for 10 and 120 minutes look-ahead, there is mean sector occupancy count over-prediction for Koksy Low on a busy and normal day. 


\subsubsection{Individual Flight Example}

In table 1.1 predictions for the British Airways example flight are given. It can be seen that predicted sector entry time, 1 hour before entry is 11:50, and this is also indicated in figure $5.13 \mathrm{a}$ by flight 1 in color cyan. The flight has been delayed, and actually enters sector Koksy High at 12:04 (figure 5.13b). For a look-ahead of 1 hour, for window 11:50 to 12:00, predicted sector occupancy count are 3 aircraft. For the same window, actual sector occupancy count is 2 aircraft.

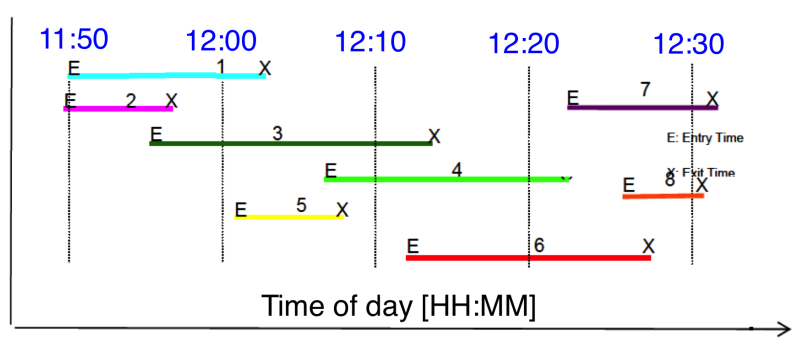

(a) Predicted flights at 1 hour look-ahead.

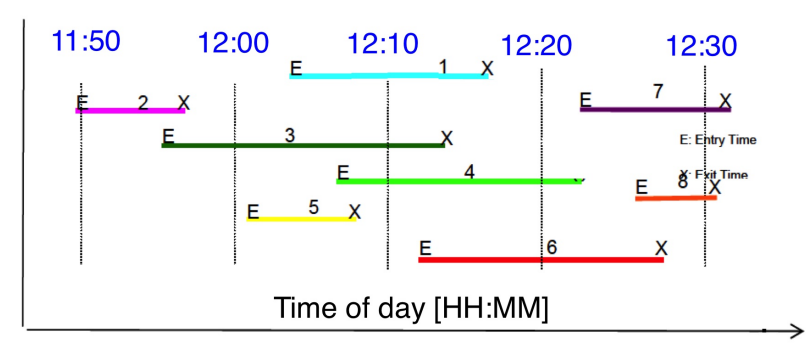

(b) Actual flights (real traffic)

Figure 5.13: Example of forecast and real occupancy count.

\subsubsection{Assumptions}

Same assumptions are made for sector occupancy count as for sector entry count in section 5.4 .

- For predicted and actual count: flights that are cancelled are excluded from the count after the time of cancellation. Before the cancellation event, these flights are still included in the predicted count.

- For predicted and actual count: flights that change trajectory and do not enter the sector are excluded after the time of trajectory change. Before the trajectory change these flights are still included in the predicted count.

- For predicted and actual count: flights that have a sector crossing duration $<1$ minute are excluded. This is because the input flight data file used does not include flights that have a crossing duration $<1$ minute. It occurs that flights are predicted to enter with crossing duration $>1$ minute, but actual crossing duration $<1$ minute. Although the flight has actually entered the sector, this is not recorded by the data file. If these flights are not excluded they will show as a count error.

- For predicted count: for a certain look-ahead time, the last available prediction is taken. For the individual example flight at a 1 hour look-ahead, the last available prediction is at 1 hour and 9 minutes look-ahead (table 1.1). 


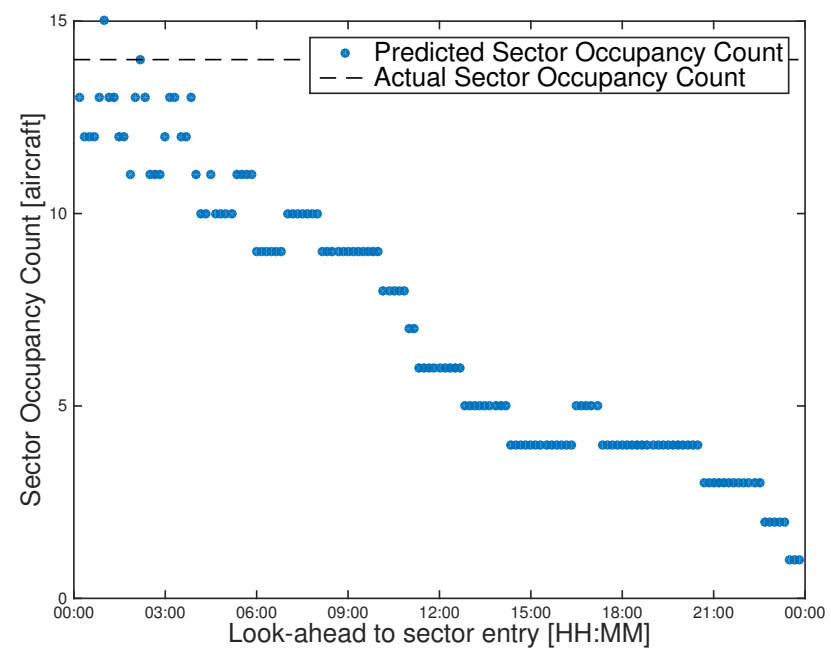

Figure 5.14: Sector occupancy count prediction for time of day window 12:00 to 12:10. Sector Koksy High

\subsubsection{Predicted Sector Occupancy Count}

Figure 5.14 shows predicted sector occupancy count as function of look-ahead, section 3.3 gives the definition of look-ahead time. Predicted count increases as we move closer to entry time. Still there is a lot of volatility in the prediction at short look-ahead times, see figure 5.15b, and for other time of day windows (figure 5.15). Table 5.4 shows the actual and predicted count for window 09:20-09:30, in terms of individual flights. Reasons for difference in predicted and actual count, are that some flights are deviated due to time \& route.

Table 5.4: Flight list of actual \& predicted sector occupancy count for window 09:20 - 09:30. In bold flights that do not occur in other column. Look-ahead 10 minutes. Sector Koksy High

\begin{tabular}{ll}
\hline $\begin{array}{l}\text { Actual occupancy } \\
\text { flight list }\end{array}$ & $\begin{array}{l}\text { Predicted occupancy } \\
\text { flight list } \\
\text { window 09:20-09:30 } \\
\text { window 09:20-09:30 } \\
\text { look-ahead = 10 min }\end{array}$ \\
\hline 94101232 & 94101232 \\
94104948 & $\mathbf{9 4 1 0 5 0 1 1}$ \\
94105761 & 94105048 \\
94117180 & $\mathbf{9 4 1 0 5 1 1 8}$ \\
94117540 & $\mathbf{9 4 1 0 5 4 8 1}$ \\
$\mathbf{9 4 1 1 7 6 5 7}$ & 94105761 \\
94119780 & 94117180 \\
94124758 & 94117540 \\
94124824 & $\mathbf{9 4 1 1 9 0 6 8}$ \\
94127675 & 94119780 \\
94134461 & 94124758 \\
& 94124824 \\
& 94127675 \\
& 94134461 \\
\hline
\end{tabular}

$\rightarrow$ flight predicted to exit at 09:25 but exits earlier at 09:19

$\rightarrow$ flight predicted to enter at 09:27, but enters at 09:31

$\rightarrow$ flight predicted to enter at 09:11 but does not enter sector

$\rightarrow$ flight not predicted to enter sector, but actually enters at 09:27

$\rightarrow$ flight predicted to enter at 09:13 but does not enter sector 


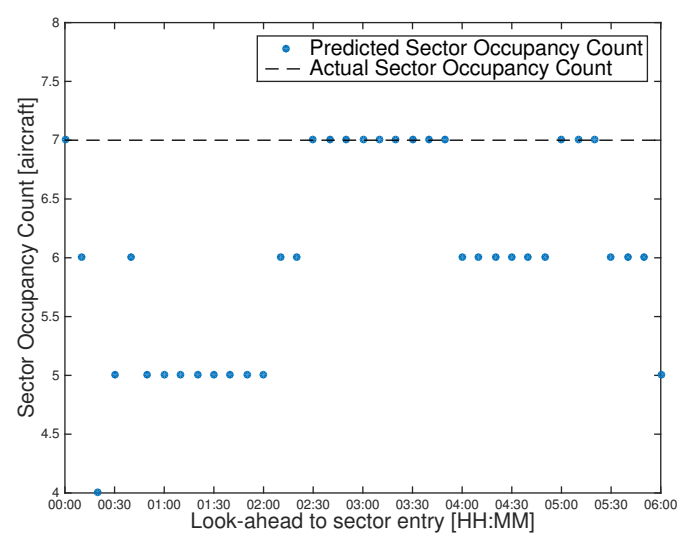

(a) Time of day window: 08:00 - 08:10

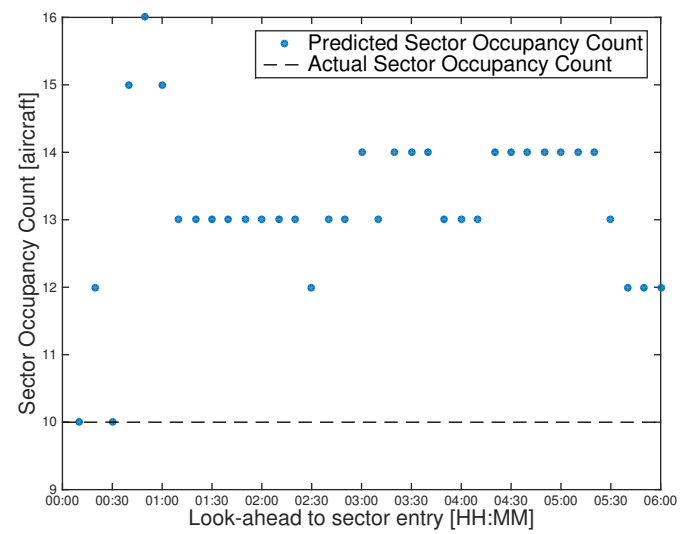

(c) Time of day window: 13:00 - 13:10

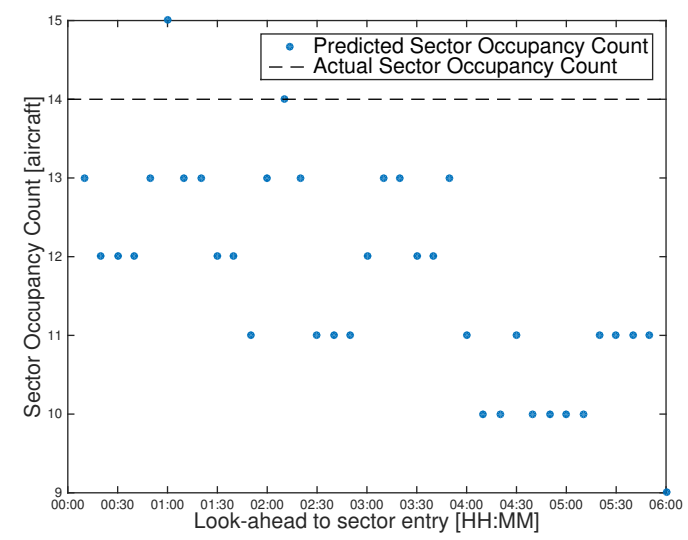

(b) Time of day window: 12:00 - 12:10

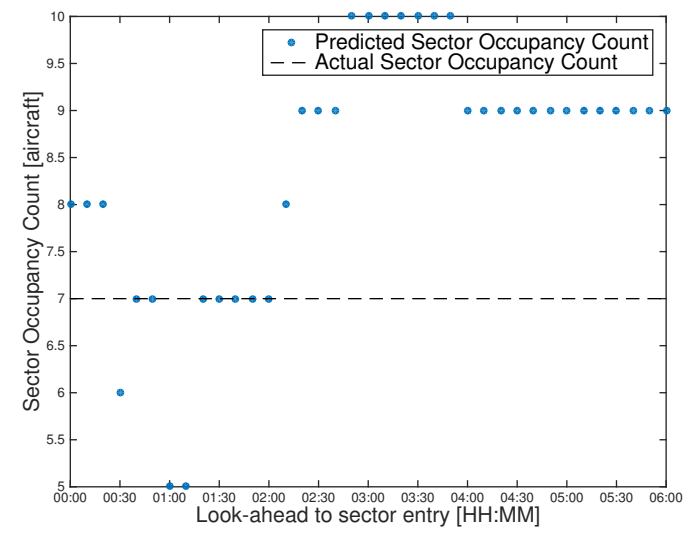

(d) Time of day window: 18:30 - 18:40

Figure 5.15: Sector occupancy count prediction for several time of day windows. Sector: Koksy High.

\subsubsection{Mean \& Standard Deviation Prediction Error}

\section{Calculation method}

In table 5.4 there were more flights predicted than flights that have actually entered. This is also the case in the example in figure 5.16a. A situation were there are more flights anticipated than really entered, is called an over-prediction. Count prediction error is calculated by subtracting actual count minus predicted count (equation 3.4). If this figure is translated to sector occupancy count prediction error, there is a negative count error. The other way around, if there are less flights forecast than real, thus an under-prediction (figure 5.16a), this is displayed in the count prediction error figures, as a positive count error. 


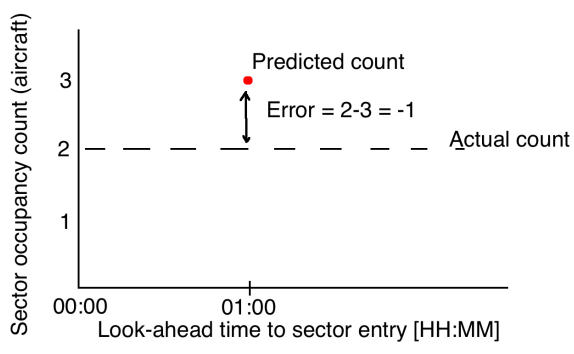

(a) Negative count error.

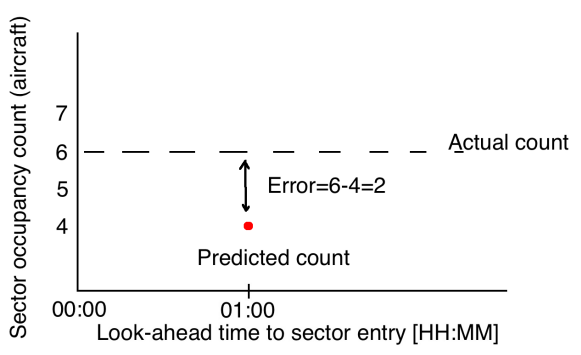

(b) Positive count error.

Figure 5.16: Example sector occupancy count prediction error for 1 hour look-ahead.

If we calculate the mean count error, taking all count prediction errors for the windows from 08:00 in the morning until 20:00 in the evening, for sector Koksy High, for a 1 hour look-ahead, this yields a count prediction error of -7 percent. This is indicated by the red dot in figure 5.17. The mean count error for all look-ahead times between 0 and 6 hours is indicated by the red line.

Note that percentage prediction error is calculated by equation 3.5. Furthermore before 08:00 and after 20:00, traffic levels are low. Percentage prediction errors at these times are out of proportion with errors for higher traffic levels. For example a prediction count error of 1 aircraft is a $50 \%$ error if the actual count is 2 aircraft, and a $2 \%$ error if the actual count is 50 aircraft.

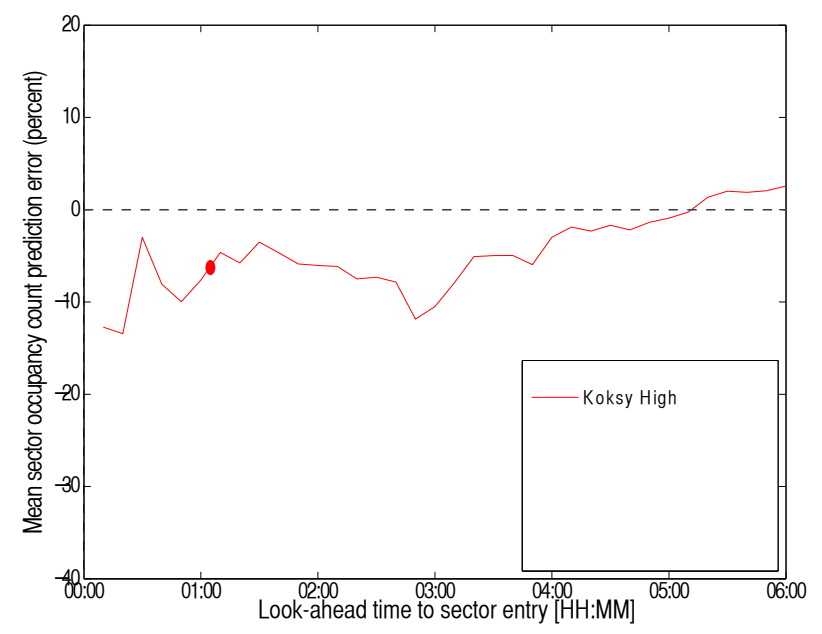

Figure 5.17: Mean sector occupancy count prediction error. Sector Koksy High

\section{Individual Sector Prediction Error}

In the same manner as mean count error is calculated, so is standard deviation of the count error calculated. Figure 5.18 show the mean and standard deviation of sector occupancy count prediction error. The percentage prediction error (figure 5.18b) is closer to 0 than aircraft prediction error (figure 5.18a). 


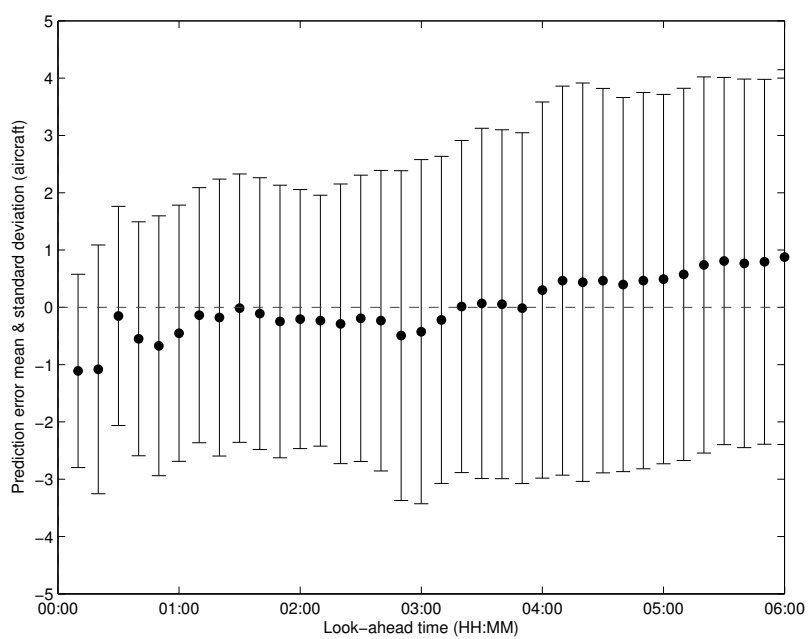

(a) Aircraft prediction error.

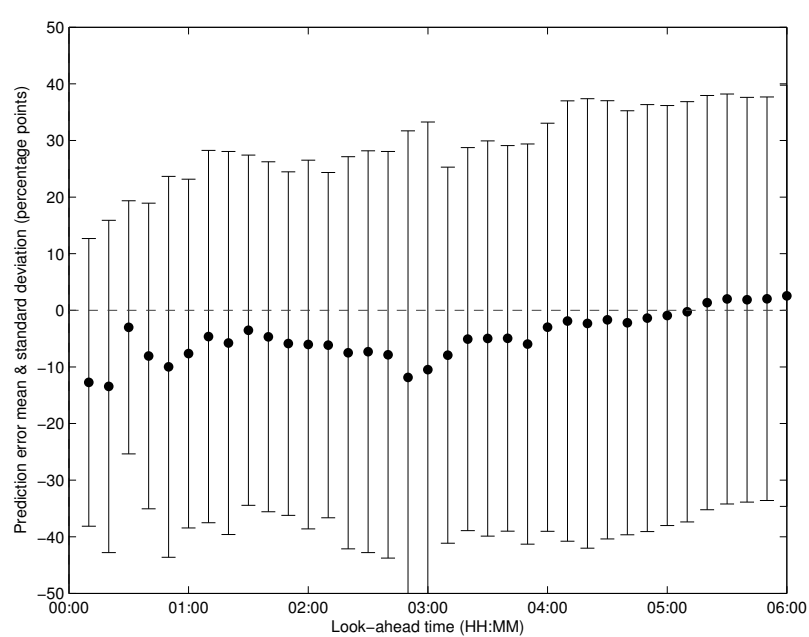

(b) Percentage prediction error.

Figure 5.18: Mean \& standard deviation sector occupancy count prediction error. Dot is mean, $1 / 2$ bar length is 1 standard deviation. Sector Koksy High.

Note that a zero count prediction error does not necessarily imply good prediction performance. It could be the case that the ETFMS correctly predicts the number of flights that will occupy a sector during a time window, but the prediction could be based on the wrong flights.

\section{Multiple Sector Prediction Error}

Previously described was count prediction error for a single sector, this subsection compares count prediction error for multiple sectors. Remarkable for 0 to 3 hours look-ahead, mean count prediction error for all sectors is below 0 (over-prediction) and fairly stable (figure 5.19b and 5.20a). Furthermore for a 6 hour look-ahead, all sectors have a mean count under-prediction, this is because not all flight plans have been filed yet (see figure 5.11). Section 5.3.9 describes the reason why mean prediction error does not go to 0 at a 10 minute look-ahead.

From figures $5.19 \mathrm{~b}$ and $5.20 \mathrm{~b}$ we can see that standard deviation of the count prediction error decreases by approximately $10 \%$ in the hour just before sector entry. Standard deviation count prediction error is fairly stable from 1 to 6 hours look-ahead. Taking prediction errors for all sectors, and calculating the mean \& standard deviation, yields the benchmark curve. This curve allows for indication of relative sector performance. It can be seen that sectors with more (percentage) over-prediction have more dispersed count prediction error.

Now looking at the mean \& standard deviation of the prediction error for the benchmark curve (figure 5.20). For a 1 hour look-ahead, the mean is $\sim-15 \%$ and standard deviation is $\sim 40$ percentage points. This means that $68 \%$ of the calculated count prediction errors are spread between $-55 \%$ and $+25 \%$, this is a large dispersion. 


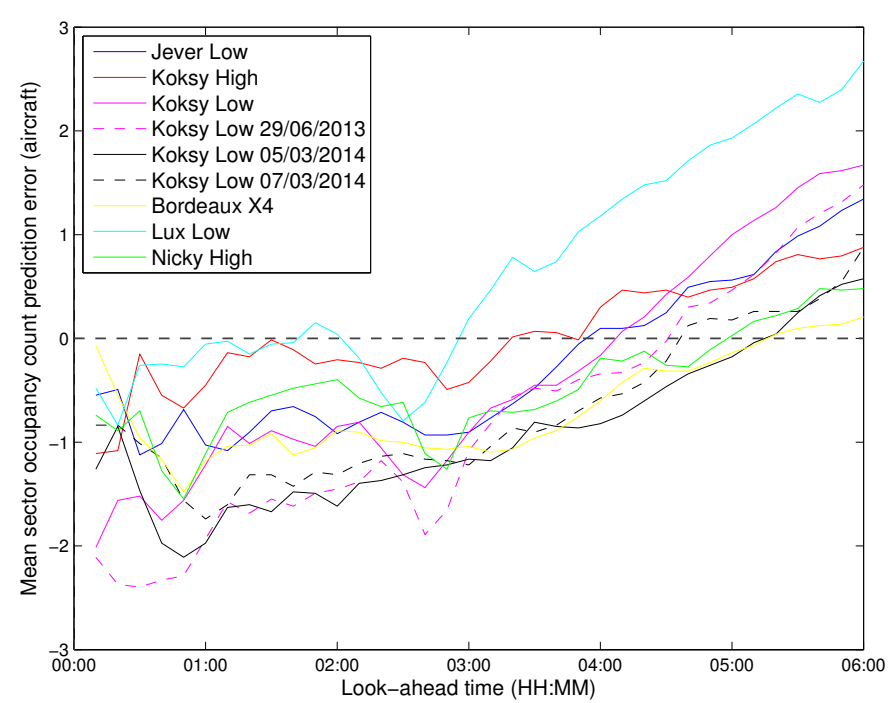

(a) Mean

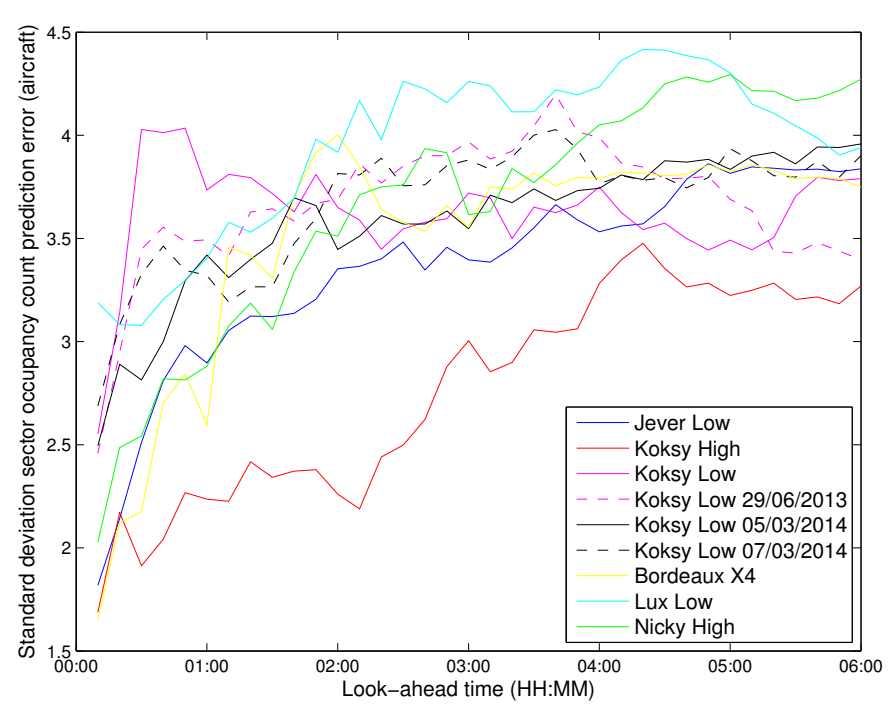

(b) Standard deviation

Figure 5.19: Sector occupancy count prediction error in aircraft, for multiple sectors. Day is 28th of June 2013 unless stated otherwise.

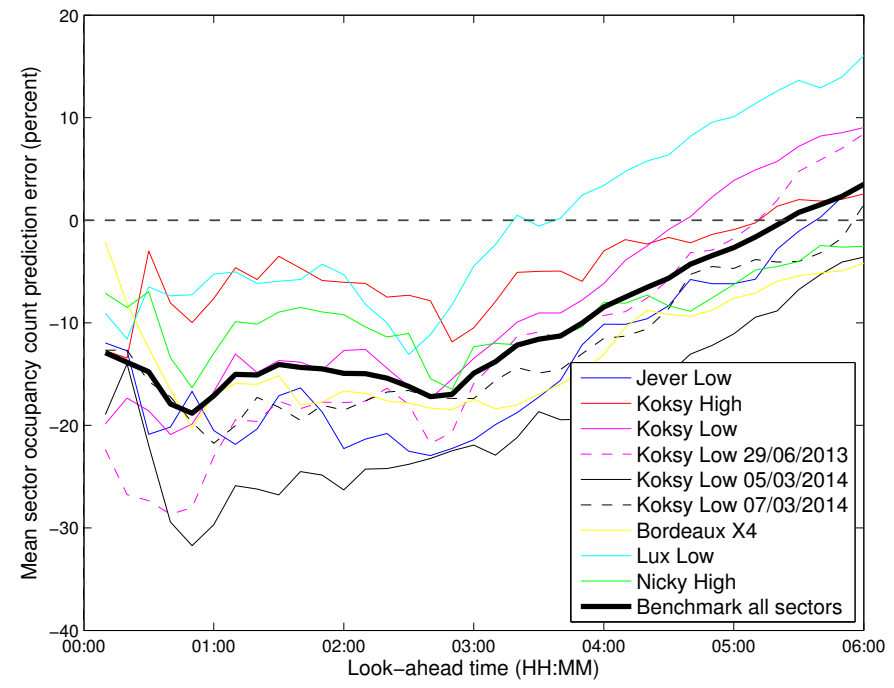

(a) Mean

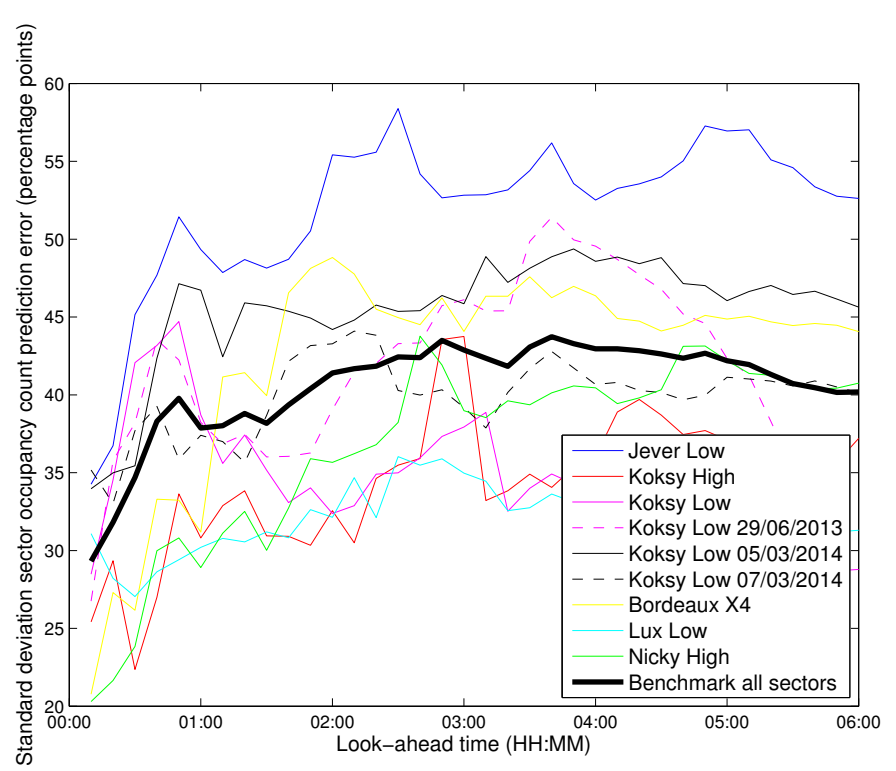

(b) Standard deviation

Figure 5.20: Sector occupancy count prediction error in percentage points, for multiple sectors. Day is 28th of June 2013 unless stated otherwise.

\subsubsection{Maximum Count Threshold}

It is apparent from figure 1.1 that there is a mismatch between forecast and real workload. For operational use, capacity is often measured in occupancy count. Turning now to quantified evidence in terms of occupancy count in figure 5.21, the actual maximum 
count is higher than predicted maximum count. This holds also for a look-ahead of 10 minutes, section 5.3.8 explores this concept more elaborately.

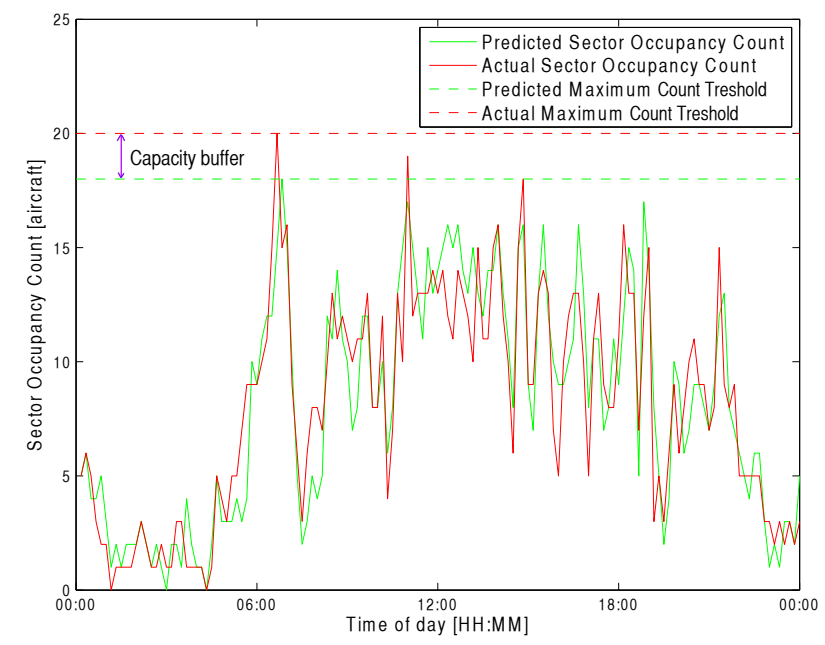

Figure 5.21: Predicted and actual sector occupancy count for 1 hour look-ahead. Sector Koksy High.

\subsubsection{Flight Status at Prediction}

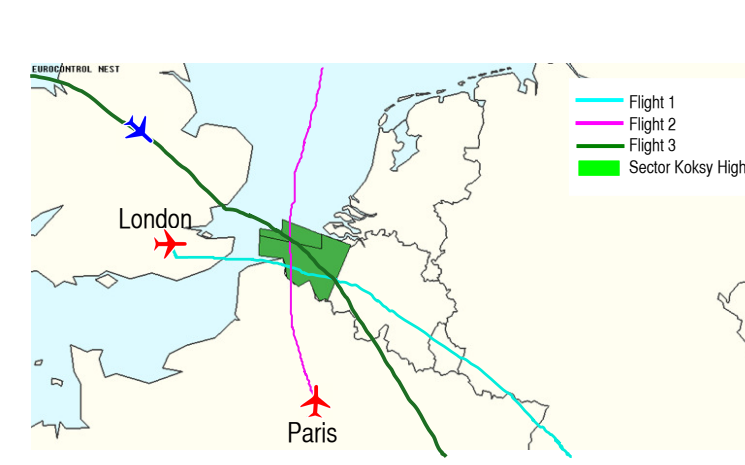

Figure 5.22: Example snapshot of predicted traffic at 1 hour look-ahead.

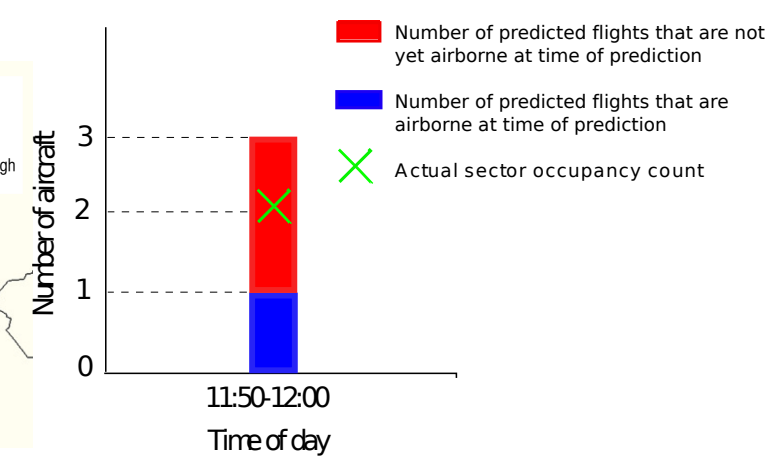

Figure 5.23: Example stacked bar flight status at prediction, based on traffic in figure 5.22

In the previous subsection we compared predicted and actual count, we now turn to differentiating the predicted count between flight statuses at the prediction (airborne or not yet airborne). To explain this concept, take traffic snapshot figure 5.22. There are 3 aircraft predicted to fly through the sector between 11:50 and 12:00, of which flight 1 is the British Airways example flight. At this look-ahead time, 1 flight is airborne, and 2 flights have not yet departed. The stacked bar chart in figure 5.23 represents these flights. If we now turn to actual sector occupancy count it appears that only 2 aircraft have occupied the sector at this window (green cross). This is because flight 1 (table 1.1) is delayed and enters the sector at 12:04. Take note that the top of the stacked bar, red and blue combined, represents the total predicted count. 


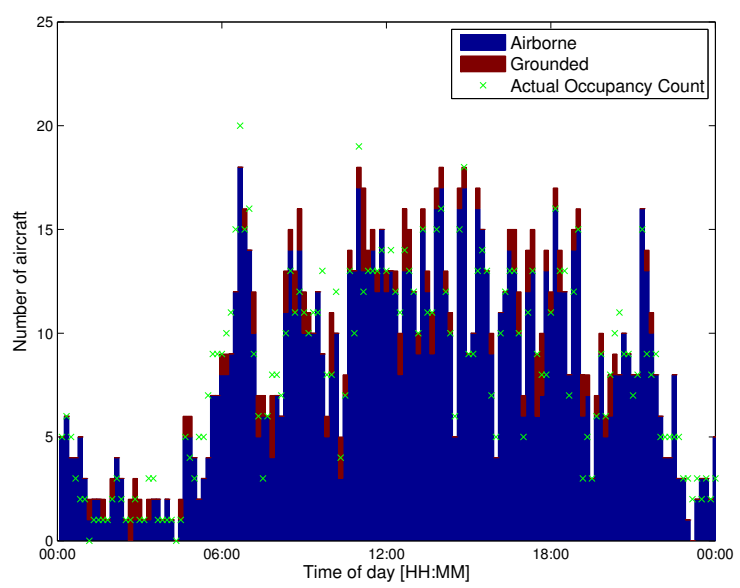

(a) Look-ahead 10 minutes.

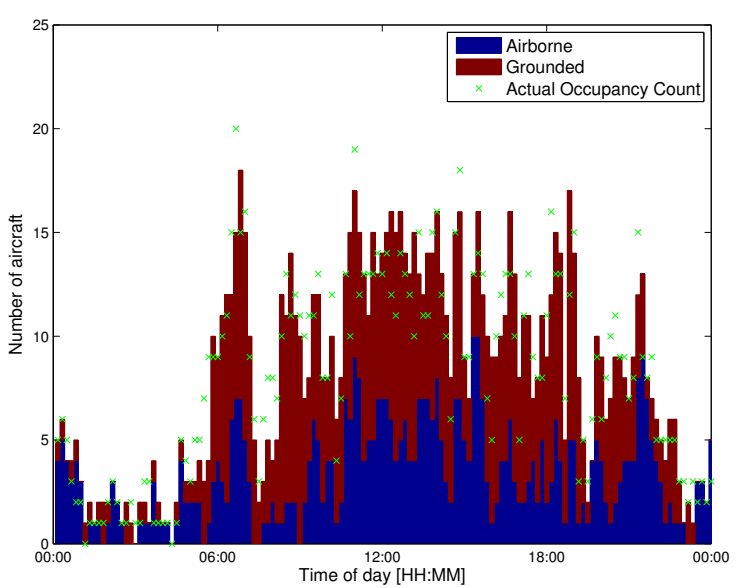

(b) Look-ahead 1 hour

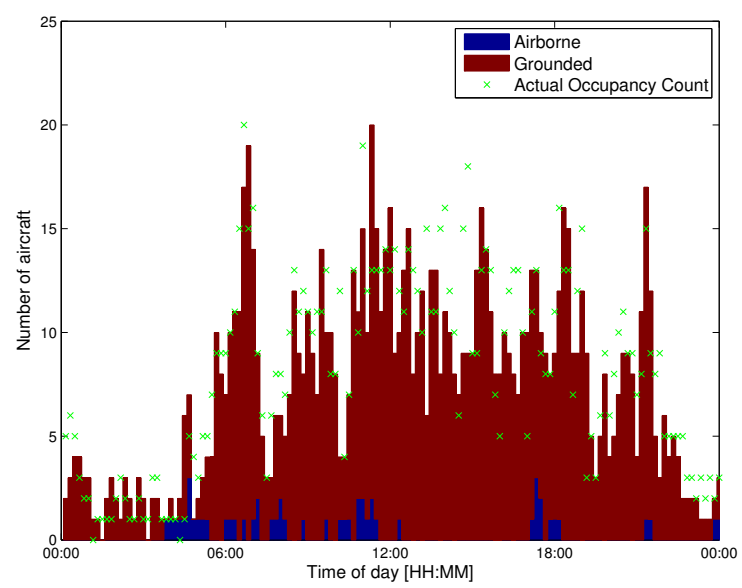

(c) Look-ahead 6 hours.

Figure 5.24: Sector occupancy count flight status at prediction. Sector Koksy High

Now turning to real data for sector Koksy High for different look-ahead times yields figure 5.24. Appendix $\mathrm{C}$ includes these figures for multiple sectors. As expected, a comparison between different look-ahead times reveals that more flights are airborne as look-ahead time decreases. The most striking result to emerge from the figures for all sectors is that the difference between predicted and real count is large, even at a look-ahead of 10 minutes.

\subsubsection{Inflow \& Outflow Flights}

In response to the question in section 5.3.6, why count prediction error is not 0 at 10 minutes look-ahead, this section aims to shine more light on this case. First terms inflow, outflow, and stable flights are explained. In figure 5.25 the predicted and actual flight occupancy times for the individual British Airways example are given. For window 11:50 to 12:00 the flight is predicted to occupy the sector, but the flight has been delayed. This results in the flight not entering in this time window. Hence for this window, the flight 


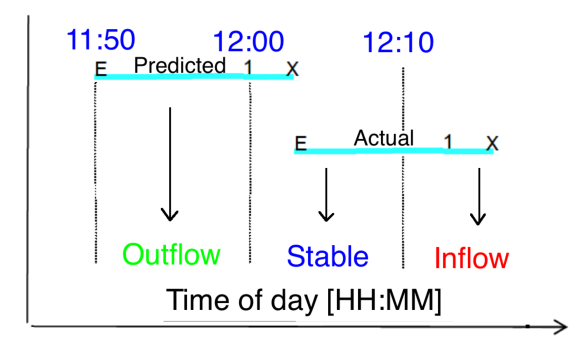

Figure 5.25: Example outflow, stable, and inflow scenarios for individual flight. Look-ahead is 1 hour

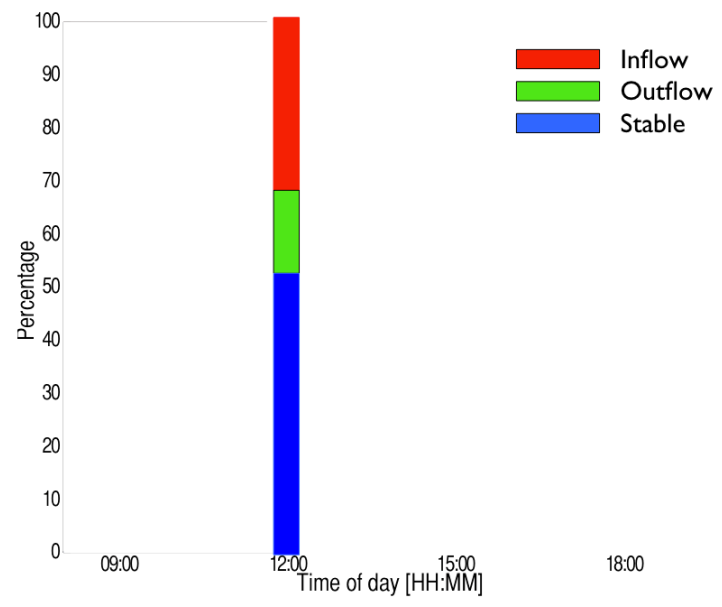

Figure 5.26: Sector occupancy count inflow, outflow, and stable flights for time of day window 11:50 - 12:00. Lookahead is 1 hour. Sector Koksy High.

is called an outflow flight. For window 12:00 to 12:10, the flight was both predicted and has actually entered in that window, and is called a stable flight. Concordantly an inflow flight is a flight that was not predicted to enter but actually enters, for a specific time window. Inflow and outflow flights can be caused by to time deviations, route deviations, late flight plan filing, or cancellations. This differentiation is quantified in section 5.3.10.

A stacked bar chart was used to analyze the percentage of inflow, outflow, and stable flights. Figure 5.26 presents the results for a single time of day window. Figure 5.27 provides more information on inflow, outflow, and stable flight percentages, for all time of day windows, for different look-ahead times. Appendix D shows these inflow figures for multiple sectors. The white box with percentages indicate the portion of inflow, outflow, or stable flights of the total summation of inflow, outflow, and stable flights. For example $12 \%$ in figure 5.27a indicate that for all inflow, outflow, and stable flights from 08:00 to 20:00, $12 \%$ are inflow flights.

It can be seen that stable flights increase as look-ahead time becomes smaller. Furthermore a relationship is revealed between mean count prediction error and inflow/outflow ratio. An over-prediction relates to less inflow flights than outflow flights (inflow/outflow $<1)$. Concordantly an under-prediction relates to more inflow flights than outflow flights (inflow/outflow > 1). For a 1 hour look-ahead the ratio for Koksy High is 0.96 (figure $5.27 \mathrm{~b}$ ), and for Koksy Low the ratio is 0.68 (figure 5.28). As can be seen in figure 5.29, Koksy Low has more over-prediction than Koksy High.

It can be reasoned algebraically that an over-prediction relates to a situation were inflow flights < outflow flights. Take figure $5.37 \mathrm{c}$, for a 40 minute look-ahead there are 8 inflow flights and 10 outflow flights, hence predicted count (54 flights) is larger than actual count (52 flights). 


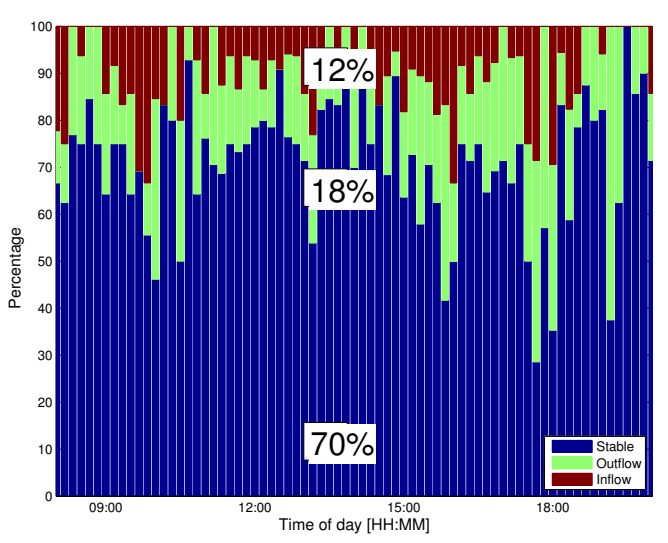

(a) Look-ahead 10 minutes.

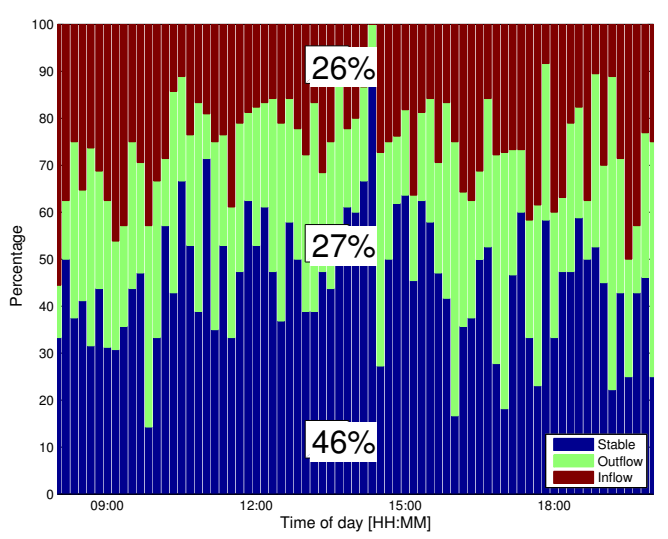

(b) Look-ahead 1 hour.

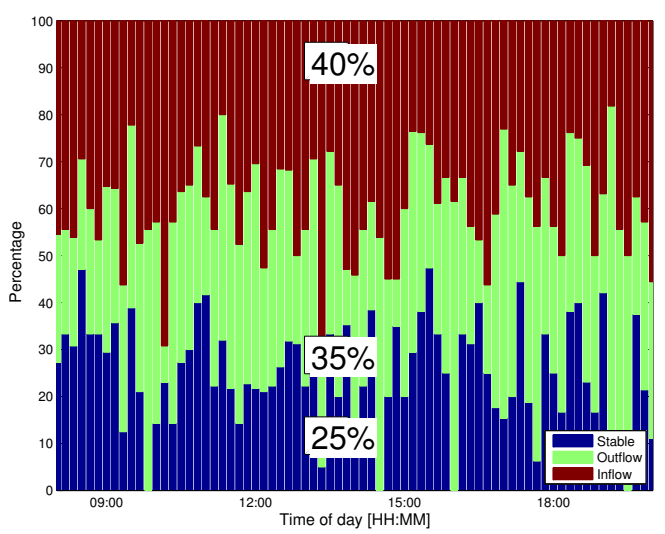

(c) Look-ahead 6 hours.

Figure 5.27: Sector occupancy count inflow, outflow, and stable flights between 08:00 and 20:00, for different look-ahead times. Sector Koksy High.

\subsubsection{Reasons Inflow \& Outflow Flights}

Several reasons that can be appointed to inflow \& outflow flights are itemized below. Table 5.5 gives a quantification of these reasons. This quantification is done for 3 different look-ahead times and all analyzed sectors in this chapter.

It is expected that an outflow flight due to time deviation for one time window, will be an inflow flight due to time deviation in another time window. Unfortunately this is not the case in table 5.5 as "outflow flights due to time deviation" $\neq$ "inflow flights due to time deviation". This inequality can be explained by looking at figure 5.25. Imagine that actual entry time and exit time fall between 12:00 and 12:10. This means that this flight is an outflow flight but no inflow flight.

In table 5.5, for inflow and outflow flights, the following differentiations are made:

- Inflow due to route deviation: these flights initially did not plan to fly trough the sector at the look-ahead time, but changed trajectory and actually entered the sector. 


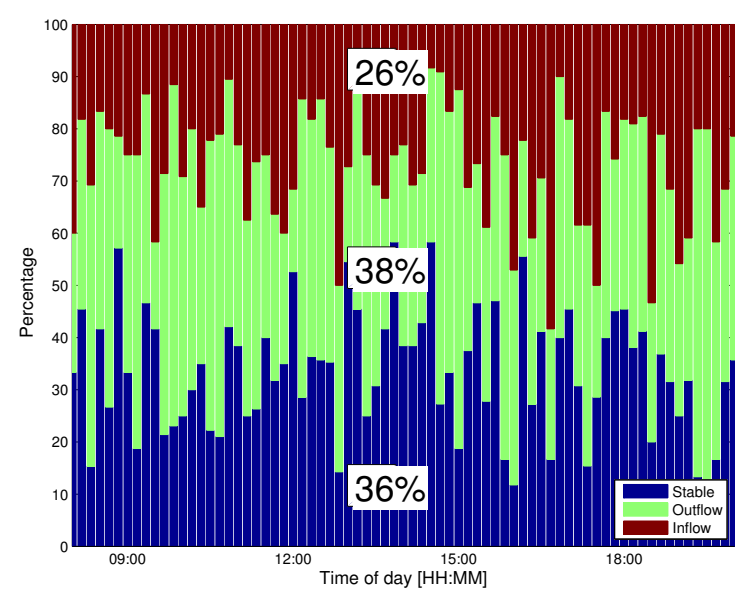

Figure 5.28: Sector occupancy count inflow, outflow, and stable flights for 1 hour lookahead. Sector Koksy Low on 5th of March 2014

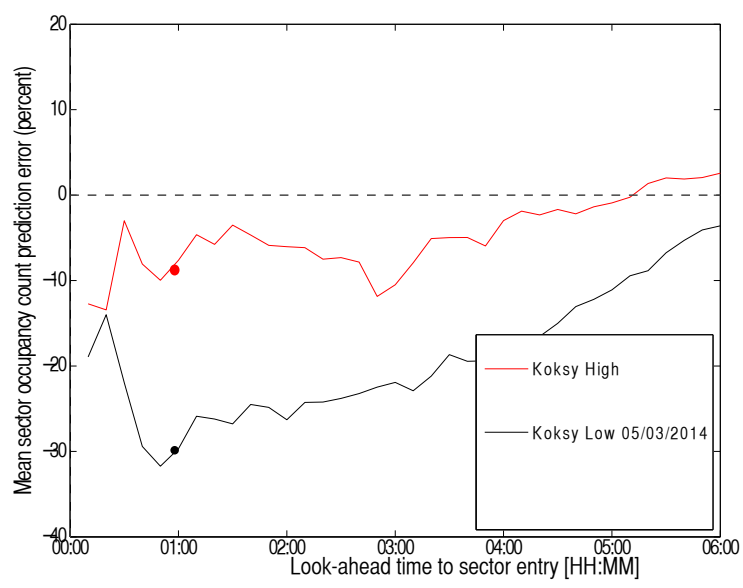

Figure 5.29: Mean sector occupancy count relative overprediction, extracted from figure $5.20 a$

- Inflow due to time deviation: these flights are not predicted to occupy a specific time window, but because the flight is delayed or earlier, the flight has actually occupied that time window.

- Inflow due to flight plan filing: these flights have not yet filed the flight plan at the regarded look-ahead time, but did enter the sector (figure 5.31).

- Outflow due to route deviation: these flights are predicted to enter the sector at a specific look-ahead time, but changed trajectory and did not enter the sector anymore (figure 5.30).

- Outflow due to time deviation: these flights are predicted to occupy a specific time window, but because the flight is delayed or earlier than predicted, the flight did occupy that specific time window.

- Outflow due to route cancellation: these flight were planned to fly through the sector, but due to a flight cancellation they did enter the sector.

- Stable flights: at a look-ahead time these flights where predicted to occupy a time window, and actually occupied that time window.

A comparison between sectors with large over-prediction and little over-prediction reveals that these sectors mainly differ in terms of inflow \& outflow due to route deviations. The sector with large over-prediction (Koksy Low 05/03/2014) has twice as much 'outflow due to route deviation' flights than the sector with little over-prediction (Lux Low). Also Koksy Low 05/03/2013 has half as much 'inflow due to route deviation' than Lux Low. It can be argued that 'outflow flights due to route deviation' should be decreased as (i) this is obtainable by ATC procedures if the situation permits, and (ii) reduces mean count prediction error, and (iii) increases the stable flights. Sector performance should be analyzed individually before undertaking action. 


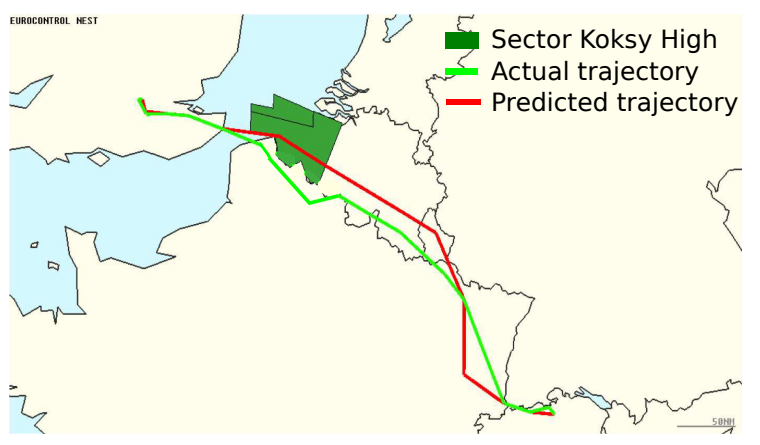

Figure 5.30: Example outflow flight due to route deviation.
$11: 50 \quad 12: 00 \quad 12: 10$

No predictions earlier than 01:54 hour look-ahead...

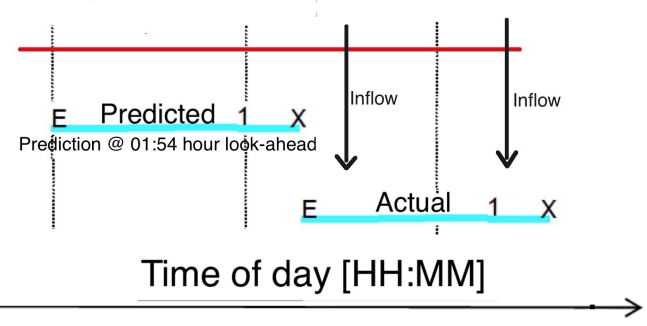

Figure 5.31: Example inflow flight due to late flight plan filing for individual flight. 


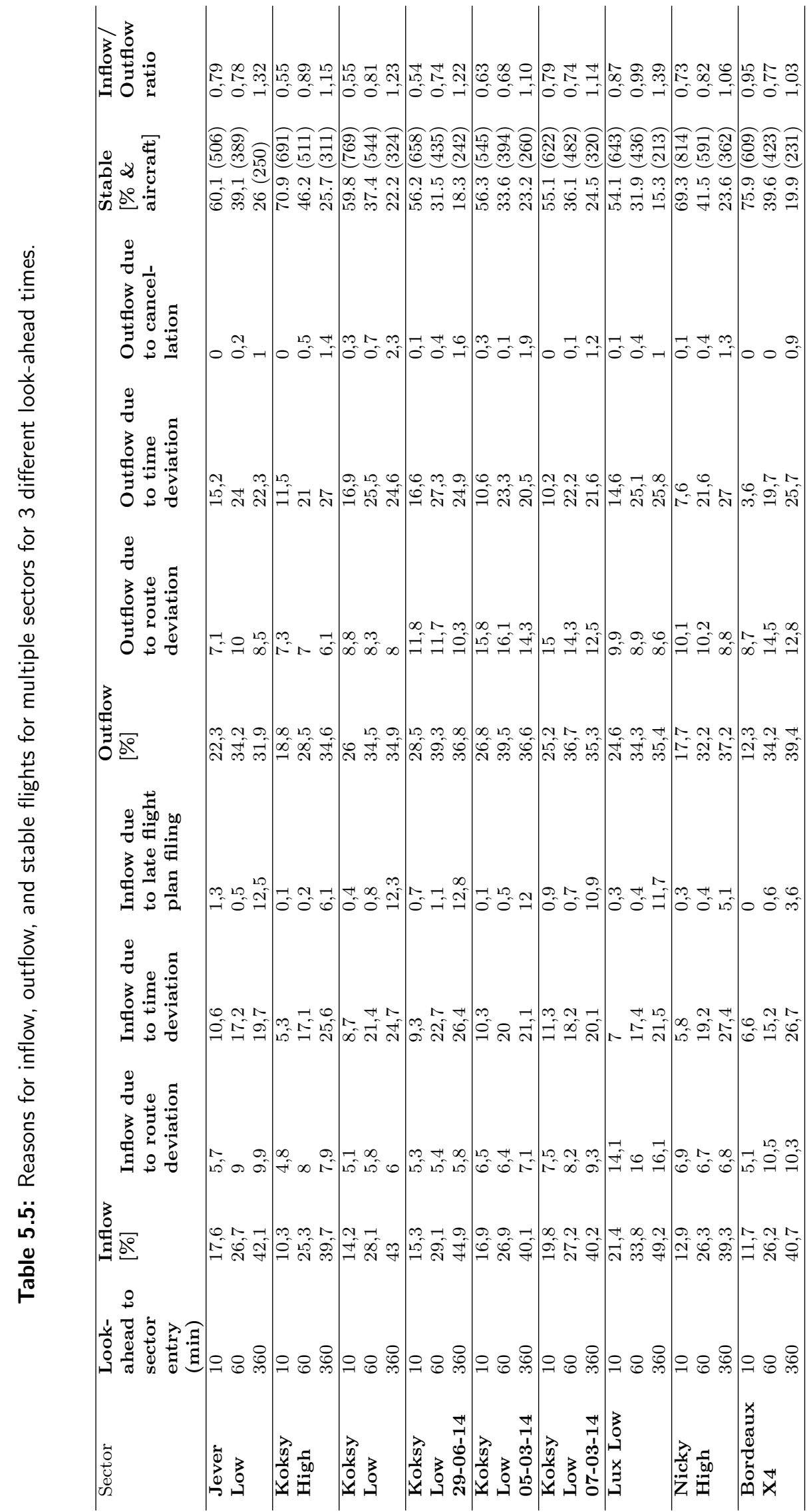




\subsection{Sector Entry Count Predictability}

This section discusses entry count in similar fashion as occupancy count (section 5.3). First a definition of entry count is given. The uncertainty is discussed by quantification of the count prediction error. Then a comparison is made between predicted and actual count, then count volatility is discussed by inflow \& outflow flights.

\subsubsection{Definition}

Sector entry count is the number of flights entering during a selected hourly time period (window). The step duration is 20 minutes, indicating the time difference between two consecutive windows. See figure 5.32

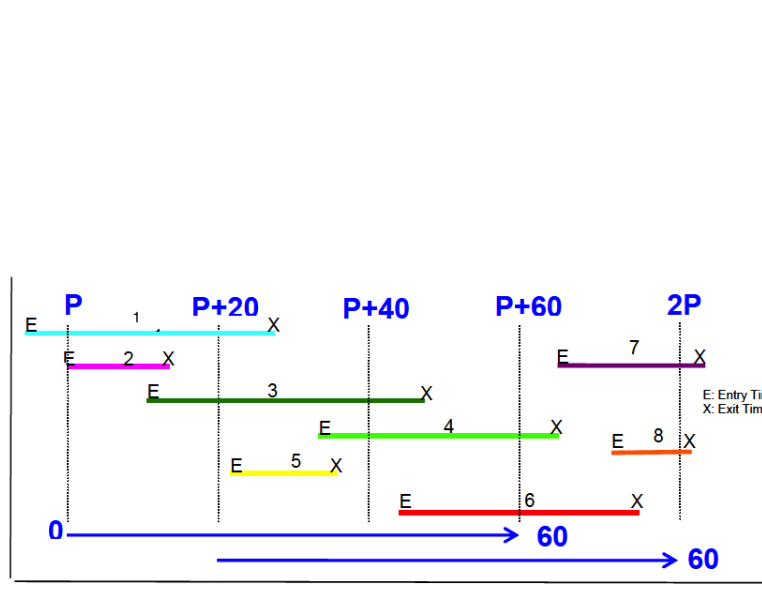

Figure 5.32: Example entry count. Skip time $=20$ minutes and window $=60$ minutes.

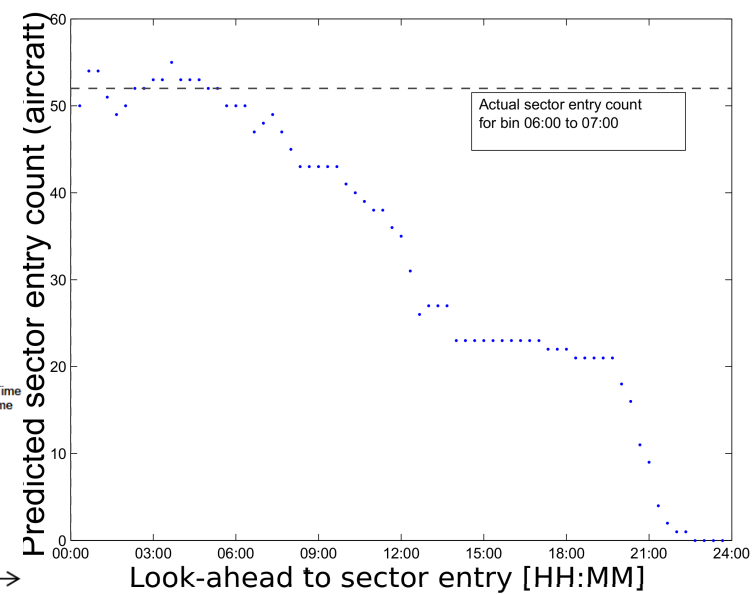

Figure 5.33: Sector entry count predictions for 06:00 to 07:00. Sector Koksy High.

In figure 5.32 at the hourly window $\mathrm{P}$ until $\mathrm{P}+60$, the entry count is 5 aircraft. These are flights; $2,3,4,5$, and 6 . Figure 5.33 shows predicted sector entry count as a function of look-ahead time. At 6 hours before sector entry, the predicted count stabilizes. At 20 minutes before entry there is still a mismatch between the predicted and actual count. Table 5.6 shows this mismatch and gives an explanation for every mismatch. 
Table 5.6: List of actual and predicted flights for window 06:00 to 07:00. In bold flights that do not occur in the other column.

\begin{tabular}{|c|c|c|}
\hline $\begin{array}{l}\text { Actual entry } \\
\text { flight list } \\
\text { window 06:00-07:00 }\end{array}$ & $\begin{array}{l}\text { Predicted entry } \\
\text { flight list } \\
\text { window 06:00-07:00 } \\
\text { (look-ahead = 00:20) }\end{array}$ & \\
\hline 94099038 & 94099038 & \\
\hline 94099673 & 94099673 & \\
\hline 94099867 & 94099867 & \\
\hline 94100059 & 94100059 & \\
\hline 94100133 & 94100133 & \\
\hline 94100147 & 94100147 & \\
\hline 94100150 & 94100150 & \\
\hline 94100173 & 94100173 & \\
\hline 94100213 & 94100213 & \\
\hline 94100217 & 94100217 & \\
\hline 94100858 & 94100858 & \\
\hline 94100935 & 94100935 & \\
\hline 94100937 & 94100937 & \\
\hline 94101361 & 94101361 & \\
\hline 94101525 & 94101525 & \\
\hline 94101716 & 94101716 & \\
\hline 94101773 & 94101773 & \\
\hline 94102281 & 94101940 & $\rightarrow$ flight predicted to enter at $06: 56$, but is delayed to $07: 07$ \\
\hline 94102453 & 94102281 & (due to ATFM regulations) \\
\hline 94102645 & 94102453 & \\
\hline $94103800-$ & $94102645 \longrightarrow$ & $\rightarrow$ flight not predicted to enter sector, but actually enters at $06: 14$ \\
\hline 94106136 & 94106136 & \\
\hline 94110143 & 94110143 & \\
\hline 94110346 & 94110346 & \\
\hline 94110460 & 94110460 & \\
\hline $94111752-$ & $94112287 \longrightarrow$ & $\rightarrow$ flight not predicted to enter sector, but actually enters at 06:01 \\
\hline 94112287 & 94112510 & $\rightarrow$ flight is predicted at 06:01, but enters earlier at 05:54 \\
\hline 94112893 & 94112893 & \\
\hline 94112898 & 94112898 & \\
\hline 94112934 & 94112934 & \\
\hline 94112946 & 94112946 & \\
\hline 94112992 & 94112992 & \\
\hline 94113342 & 94113342 & \\
\hline $94113651-$ & $94113846 \longrightarrow$ & $\rightarrow$ flight not predicted to enter sector, but actually enters at 06:50 \\
\hline 94113846 & 94113905 & \\
\hline 94113905 & 94113966 & \\
\hline 94113966 & 94115103 & \\
\hline 94115103 & 94116175 & \\
\hline 94116175 & 94116461 & $\rightarrow$ flight predicted to enter at $06: 55$, but is delayed to $07: 05$ \\
\hline 94116566 & 94116566 & \\
\hline $94116663-$ & $94117116 \longrightarrow$ & $\rightarrow$ flight not predicted to enter sector, but actually enters at 06:56 \\
\hline 94116730 & $94118038 \longrightarrow$ & $\rightarrow$ flight predicted to enter at $05: 58$, but is delayed to $06: 00$ \\
\hline 94117116 & 94119039 & \\
\hline 94118038 & 94120170 & \\
\hline 94119039 & 94120643 & \\
\hline 94120170 & 94122223 & \\
\hline 94120643 & 94122281 & \\
\hline 94121498 & $94123835 \longrightarrow$ & $\rightarrow$ flight not predicted to enter sector, but actually enters at 06:49 \\
\hline 94122223 & 94124009 & $\rightarrow$ flight predicted to enter $06: 48$, but does not enter the sector \\
\hline 94122281 & 94125834 & \\
\hline 94123835 & & \\
\hline 94125834 & & \\
\hline
\end{tabular}




\subsubsection{Mean \& Standard Deviation Prediction Error}

Count prediction error is calculated by equation 3.4 and the procedure to obtain the mean $\&$ standard deviation of the count prediction error is given in section 5.3.6.

Figure 5.34 and 5.35 illustrate sector characteristics in terms of sector entry count prediction error. In general, from 0 to 3 hour look-ahead, there is an over-prediction, however for this period sectors Lux Low and Koksy High have an under-prediction. Interestingly, the standard deviation of percentage prediction error is fairly stable (figure 5.35b)

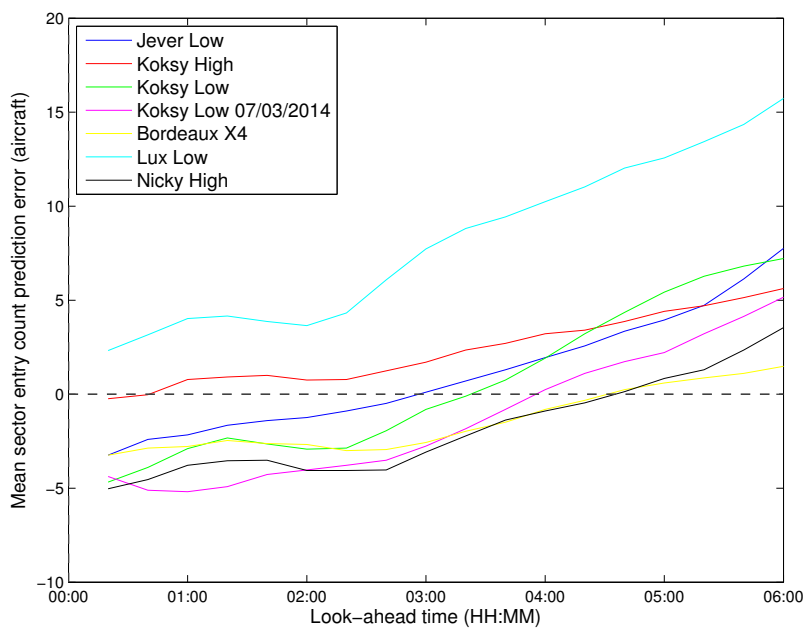

(a) Mean

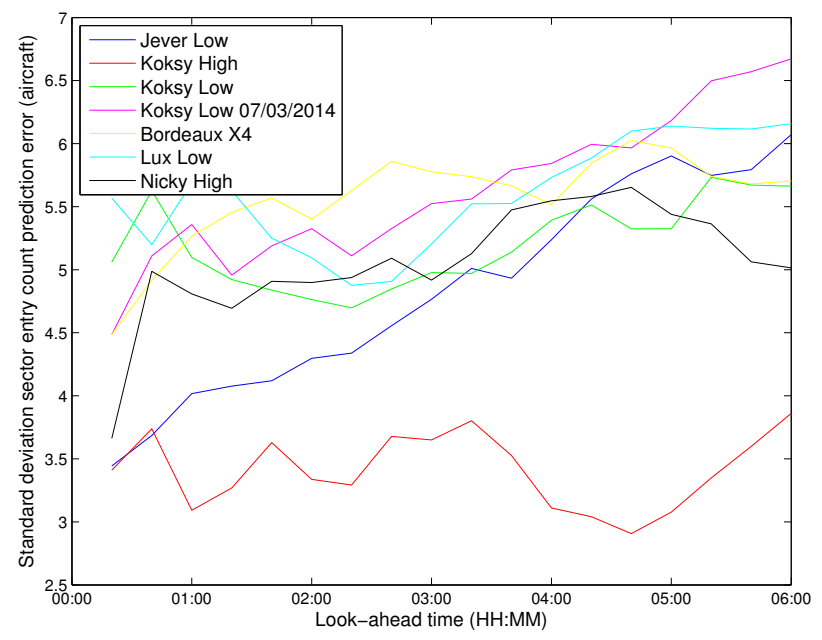

(b) Standard deviation

Figure 5.34: Sector entry count prediction error in aircraft, for multiple sectors. Day is 28th of June 2013 unless stated otherwise

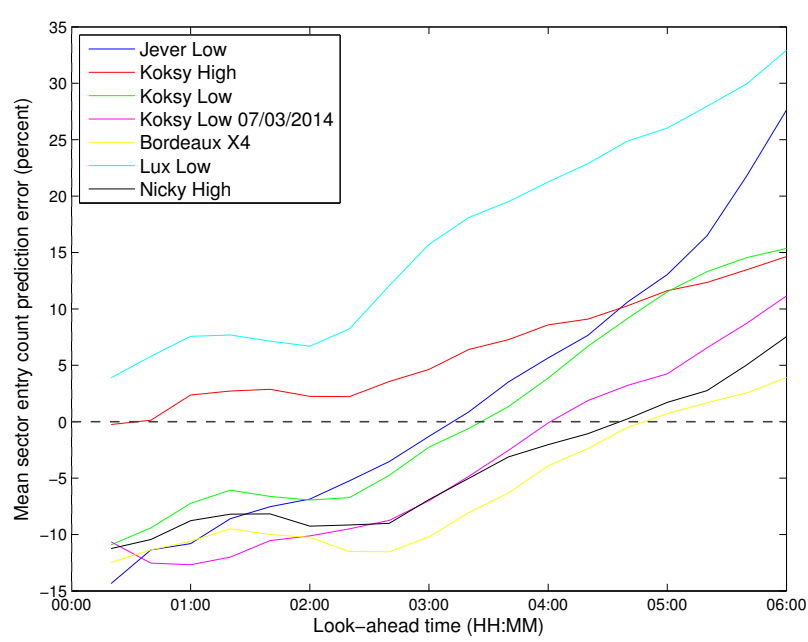

(a) Mean

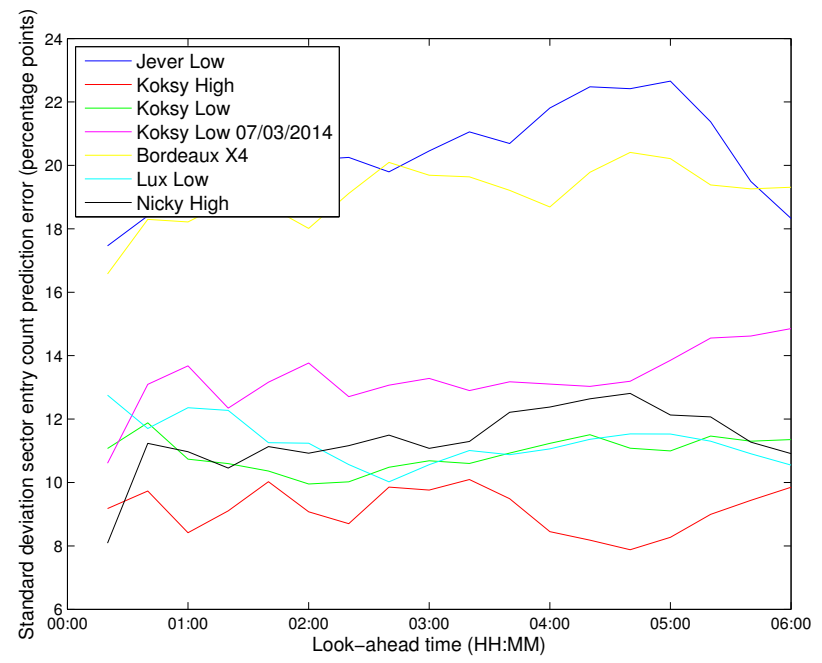

(b) Standard deviation

Figure 5.35: Sector entry count prediction error in percentage points, for multiple sectors. Day is 28th of June 2013 unless stated otherwise 


\subsubsection{Flight Status at Prediction}

Explanation of figures in this sections are given in section 5.3.8. Figure 5.36 compares actual and predicted count. For the predicted count, a differentiation is made between flights airborne, and not yet airborne at the look-ahead time. The stacked bar indicates that a large portion of the flights are still on the ground, at a look-ahead of 20 minutes. In accordance with figure 5.34a prediction error is small at a 20 minute look-ahead (figure 5.36a) and there is an under-prediction for a 6 hour look-ahead (figure 5.36c). Other sectors are also analyzed for a 1 hour look-ahead, see appendix E.

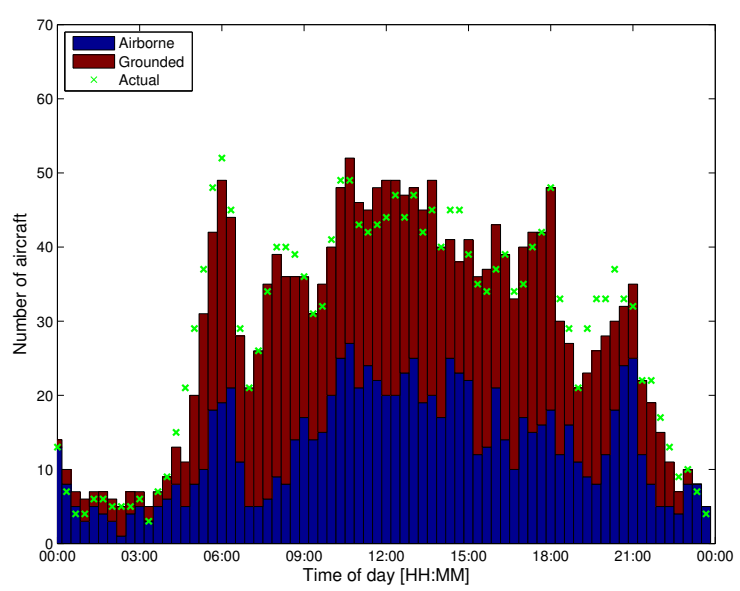

(a) Look-ahead 20 minutes.

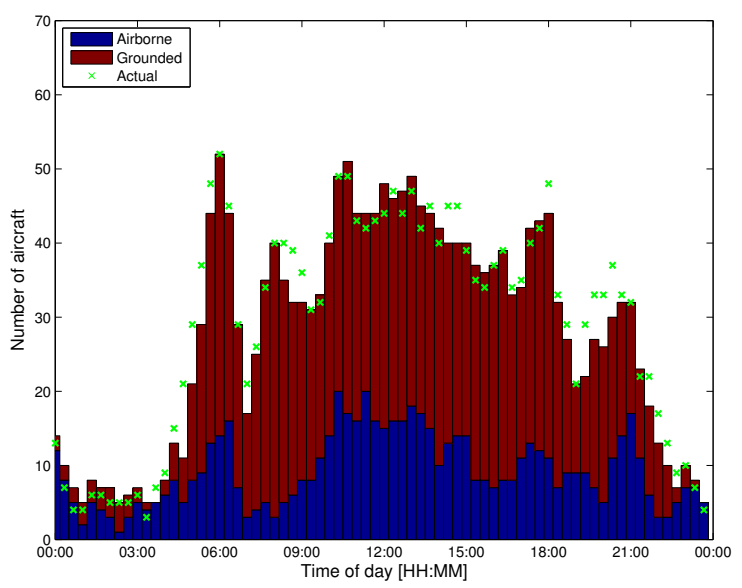

(b) Look-ahead 1 hour.

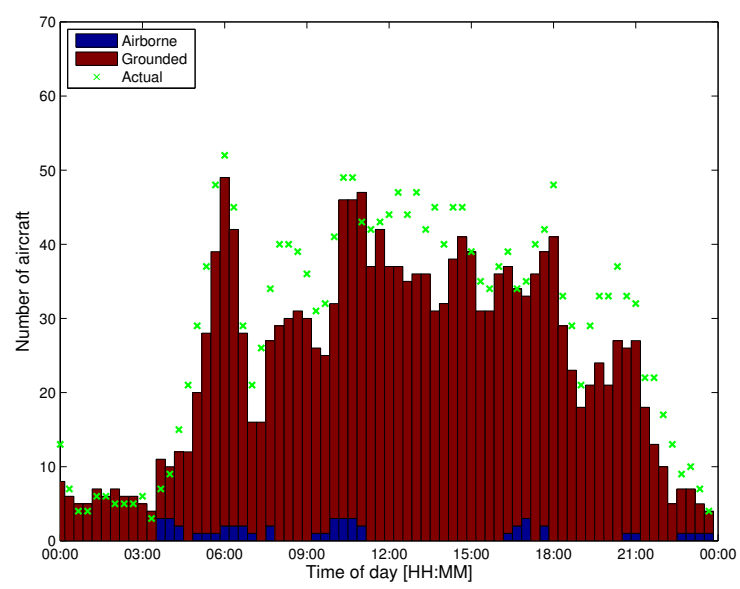

(c) Look-ahead 6 hours.

Figure 5.36: Sector entry count flight status at prediction for different look-ahead times. Sector Koksy High

\subsubsection{Inflow \& Outflow Flights}

The terms inflow, outflow, and stable flights are explained in section 5.3.9. These terms are also explained by figure 5.37a. Figure 5.37 shows prediction volatility for different 
look-ahead times, at 06:00 to 07:00. It is clear from figure 5.37 that there is large volatility for the 2 hour period before entry, especially 20 minutes before entry.

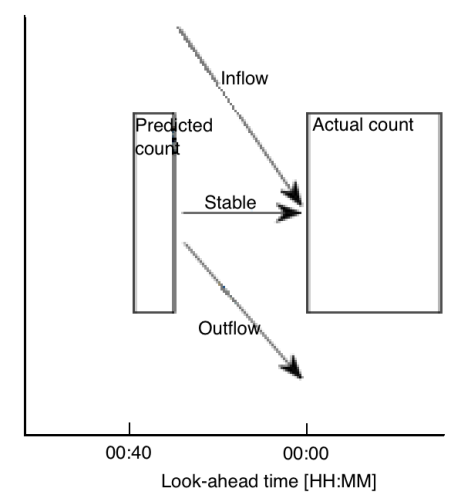

(a) Explanation terms inflow, outflow, and stable.

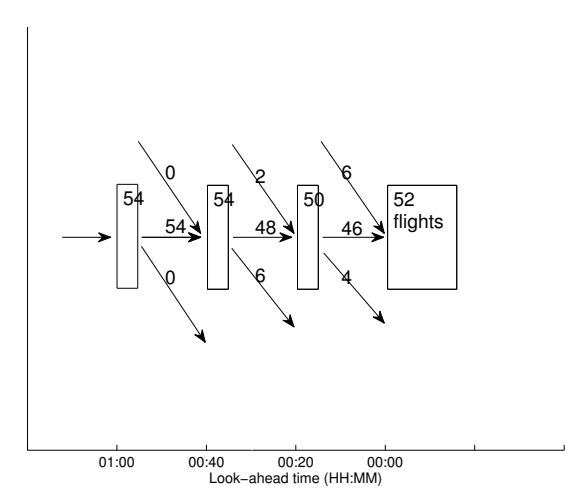

(b) Look-ahead 1 hour.

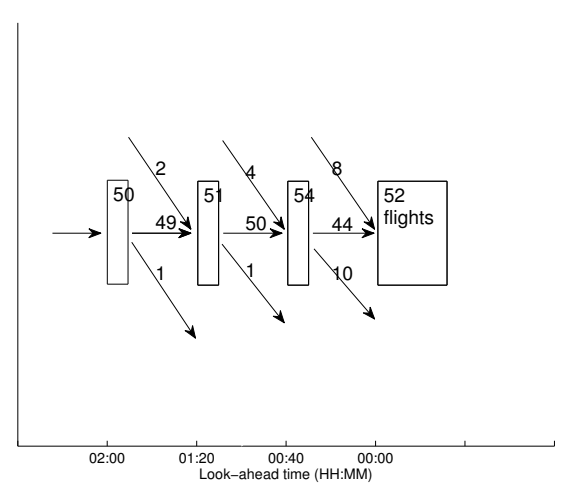

(c) Look-ahead 2 hours.

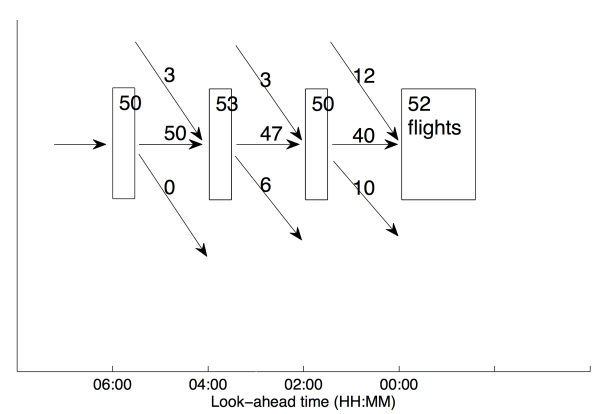

(d) Look-ahead 6 hour.

Figure 5.37: Sector entry count flow diagram for time of day window 06:00 to 07:00, for different look-ahead times. Sector: Koksy High.

Now making the transition from a single window to all windows between 08:00 and 20:00 (explained elaborately in section 5.3.9) yields figure 5.38. It can be seen that for all given look-ahead times there are more inflow flights than outflow flight, which relates to an under-prediction. This is supported by figure 5.35a, where there is indeed underprediction (positive count error) for sector Koksy High. 


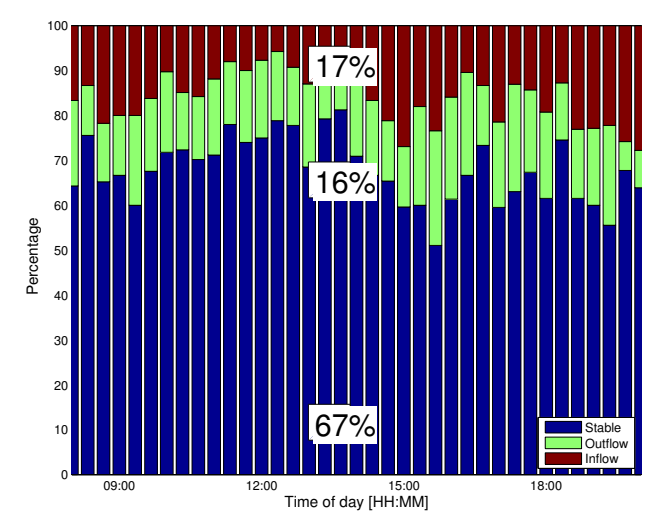

(a) Look-ahead 20 minutes.

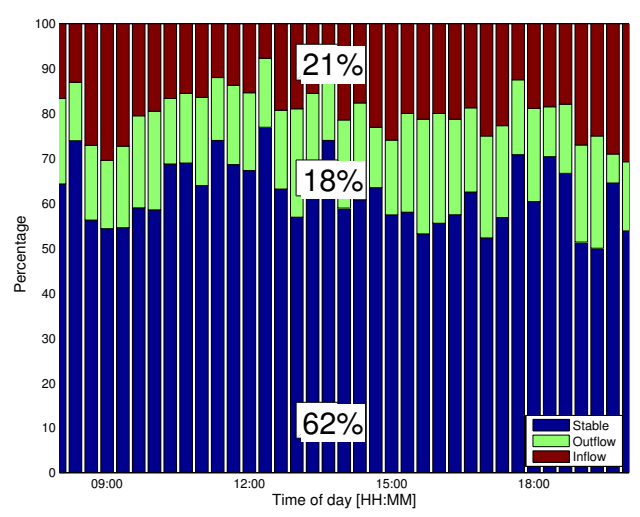

(b) Look-ahead 1 hour.

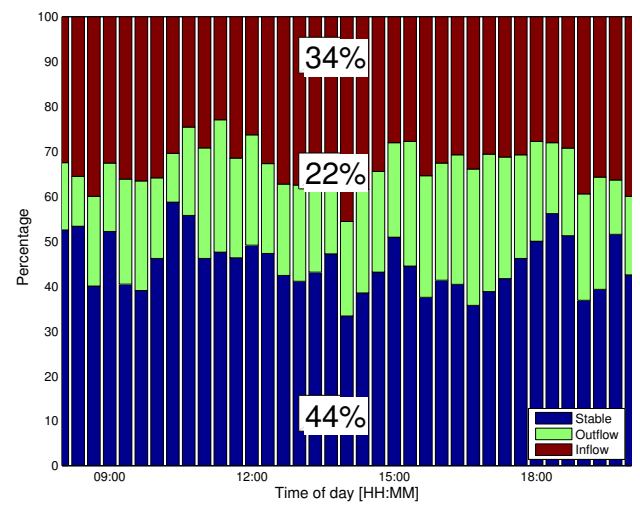

(c) Look-ahead 6 hours.

Figure 5.38: Sector entry count inflow, outflow, and stable flights between 08:00 and 20:00, for different look-ahead times. Sector Koksy High.

\subsection{Conclusions}

This section highlights the main points of the analysis on sector demand predictability:

- The function that best explains the variation in the prediction error relative to look-ahead time, is a 9th degree polynomial fit.

- Prediction error for flights with longer flight times to the sector is smaller. A possible explanation is that more time allows pilots to speed up / slow down to adhere to the planned entry time.

- On a busy day, flights are more probable to be delayed for a look-ahead longer than 2 hours.

- Absolute sector entry time prediction error (variation of the error) increases linearly from 0 to 3 hours look-ahead, then it stabilizes around 7 minutes.

- Standard deviation sector entry time prediction error increases by 10 minutes per 1 hour look-ahead. 
- At 0 to 3 hours look-ahead, all the flights that intend to enter the sector have filed the flight plan.

- Most analyzed sectors show a constant mean entry \& occupancy count over-prediction of $\sim 15 \%$ for a look-ahead of 0 to 3 hours. However, sector Koksy High and Lux Low show a mean entry count under-prediction. Over-prediction means that there are more flights anticipated than really entered.

- Mean entry \& occupancy count error are not reduced to zero just before sector entry. This is due to flights being earlier or delayed, or due to route deviations, just before sector entry. Looking at the time period from 0 to 10 minutes before entry, it can be seen that some forecasted flights did not enter in the window anymore (outflow), and some additional flights entered in the window that were initially not forecasted (inflow). Looking at 10 minutes before entry, about $30 \%$ to $40 \%$ are inflow/outflow flights, which is considered as a large amount.

- In general, for a look-ahead of 0 to 3 hours, there are more outflow than inflow flights resulting in the before mentioned over-prediction. The inflow/outflow flights ratio determines the mean sector occupancy count prediction error.

- Sectors with large over-prediction have a large number of outflow flights due to route deviation. In order to reduce over-prediction for these sectors, outflow flights due to route deviation should be reduced. For Koksy Low on the 5th of March 2014, a $5 \%$ decrease in outflow flights due to route deviations, reduces mean occupancy count prediction error by $10 \%$. As vertical route uncertainty causes $70 \%$ of the uncertainty problems on operations [5], and ensuring that safety is kept at the level of today, operationally unnecessary level changes should be reduced. 


\section{Chapter 6}

\section{Validation of Actual Traffic Count}

This chapter compares the obtained actual traffic count in the thesis with actual traffic count from the Eurocontrol software tool NEST [3]. First sector occupancy count is discussed, then sector entry count

\subsection{Sector Occupancy Count}

Section 5.3 explains how actual sector occupancy count is obtained. Table 6.3 compares the actual count that is obtained in this thesis with NEST. It can be seen that there are occurrences were NEST counts additional flights that are not counted in the algorithm for the thesis. There are 7 occurrences of such count discrepancy, which are shown in table 6.2. The reason for an extra count in NEST are because some flights enter the sector twice, this is possible due to sector geometry. The input flight data file (EFD) for the thesis algorithm only shows a single entry and single exit time, while the flight actually entered the sector more than once in NEST (real life). Table 6.1 shows such double entry scenario for one flight.

Table 6.1: Actual entry and exit times of flight AA94129641 in NEST and EFD.

\begin{tabular}{llll}
\hline Flight & Entry time & Exit time & Model \\
\hline AA94129641 & $20: 23: 00$ & $20: 33: 28$ & NEST \\
AA94129641 & $20: 34: 46$ & $20: 41: 25$ & NEST \\
AA94129641 & $20: 23: 00$ & $20: 41: 25$ & THESIS \\
\hline
\end{tabular}

The software tool NEST does not enable to view occupancy count predictions for certain look-ahead times, so it is not possible to compare the predicted sector occupancy count from this thesis algorithm.

Take note that the EFD file does not include flights which have a sector crossing duration of less than 1 minute. Therefore these flights are excluded from the predicted and actual 
count, but for explanatory reasons these flights are included in the thesis counts in table 6.3 and 6.4.

Table 6.2: Additional flights for NEST data compared with thesis algorithm (table 6.3)

\begin{tabular}{|c|c|c|c|}
\hline Start bin & End bin & Flight & Remarks \\
\hline $9: 10$ & $9: 20$ & AA94131882 & $\begin{array}{l}\text { Double entry in NEST so counted } \\
\text { twice, but only one sector entry in EFD }\end{array}$ \\
\hline $11: 50$ & 12:00 & AA94109000 & $\begin{array}{l}\text { Double entry in NEST so counted } \\
\text { twice, but only one sector entry in EFD }\end{array}$ \\
\hline $12: 20$ & $12: 30$ & AA94133541 & $\begin{array}{l}\text { Double entry in NEST so counted } \\
\text { twice, but only one sector entry in EFD }\end{array}$ \\
\hline $12: 50$ & 13:00 & AA94136975 & $\begin{array}{l}\text { Double entry in NEST so counted } \\
\text { twice, but only one sector entry in EFD }\end{array}$ \\
\hline $13: 20$ & $13: 30$ & AA94135438 & $\begin{array}{l}\text { Double entry in NEST so counted } \\
\text { twice, but only one sector entry in EFD }\end{array}$ \\
\hline $13: 50$ & 14:00 & AA94132246 & $\begin{array}{l}\text { Double entry in NEST so counted } \\
\text { twice, but only one sector entry in EFD }\end{array}$ \\
\hline $20: 30$ & $20: 40$ & AA94129641 & $\begin{array}{l}\text { Double entry in NEST so counted } \\
\text { twice, but only one sector entry in EFD }\end{array}$ \\
\hline
\end{tabular}


Table 6.3: Actual sector occupancy count comparison between NEST and thesis algorithm. Sector Jever Low.

\begin{tabular}{|c|c|c|c|c|c|c|c|c|c|}
\hline Start bin & End bin & Thesis & NEST & $\begin{array}{l}\text { Thesis - } \\
\text { NEST }\end{array}$ & Start bin & End bin & Thesis & NEST & $\begin{array}{l}\text { Thesis - } \\
\text { NEST }\end{array}$ \\
\hline $0: 00$ & $0: 10$ & 0 & 0 & 0 & $12: 00$ & $12: 10$ & 7 & 7 & 0 \\
\hline $0: 10$ & $0: 20$ & 1 & 1 & 0 & $12: 10$ & $12: 20$ & 6 & 6 & 0 \\
\hline $0: 20$ & $0: 30$ & 1 & 1 & 0 & $12: 20$ & $12: 30$ & 10 & 12 & -2 \\
\hline $0: 30$ & $0: 40$ & 1 & 1 & 0 & $12: 30$ & $12: 40$ & 6 & 6 & 0 \\
\hline $0: 40$ & $0: 50$ & 0 & 0 & 0 & $12: 40$ & $12: 50$ & 8 & 8 & 0 \\
\hline $0: 50$ & $1: 00$ & 1 & 1 & 0 & $12: 50$ & $13: 00$ & 7 & 8 & -1 \\
\hline $1: 00$ & $1: 10$ & 0 & 0 & 0 & $13: 00$ & $13: 10$ & 12 & 12 & 0 \\
\hline $1: 10$ & $1: 20$ & 0 & 0 & 0 & $13: 10$ & $13: 20$ & 10 & 10 & 0 \\
\hline $1: 20$ & $1: 30$ & 0 & 0 & 0 & $13: 20$ & $13: 30$ & 14 & 15 & -1 \\
\hline $1: 30$ & $1: 40$ & 0 & 0 & 0 & $13: 30$ & $13: 40$ & 17 & 17 & 0 \\
\hline $1: 40$ & $1: 50$ & 0 & 0 & 0 & $13: 40$ & $13: 50$ & 12 & 12 & 0 \\
\hline $1: 50$ & $2: 00$ & 0 & 0 & 0 & $13: 50$ & $14: 00$ & 13 & 14 & -1 \\
\hline $2: 00$ & $2: 10$ & 0 & 0 & 0 & $14: 00$ & $14: 10$ & 10 & 10 & 0 \\
\hline $2: 10$ & $2: 20$ & 0 & 0 & 0 & $14: 10$ & $14: 20$ & 6 & 6 & 0 \\
\hline $2: 20$ & $2: 30$ & 1 & 1 & 0 & $14: 20$ & $14: 30$ & 4 & 4 & 0 \\
\hline $2: 30$ & $2: 40$ & 1 & 1 & 0 & $14: 30$ & $14: 40$ & 4 & 4 & 0 \\
\hline $2: 40$ & $2: 50$ & 0 & 0 & 0 & $14: 40$ & $14: 50$ & 6 & 7 & -1 \\
\hline $2: 50$ & $3: 00$ & 0 & 0 & 0 & $14: 50$ & $15: 00$ & 7 & 7 & 0 \\
\hline $3: 00$ & $3: 10$ & 1 & 1 & 0 & $15: 00$ & $15: 10$ & 9 & 9 & 0 \\
\hline $3: 10$ & $3: 20$ & 2 & 2 & 0 & $15: 10$ & $15: 20$ & 9 & 9 & 0 \\
\hline $3: 20$ & $3: 30$ & 2 & 2 & 0 & $15: 20$ & $15: 30$ & 12 & 12 & 0 \\
\hline $3: 30$ & $3: 40$ & 2 & 2 & 0 & $15: 30$ & $15: 40$ & 9 & 9 & 0 \\
\hline $3: 40$ & $3: 50$ & 1 & 1 & 0 & $15: 40$ & $15: 50$ & 10 & 10 & 0 \\
\hline $3: 50$ & 4:00 & 2 & 2 & 0 & $15: 50$ & $16: 00$ & 15 & 15 & 0 \\
\hline 4:00 & $4: 10$ & 2 & 2 & 0 & $16: 00$ & $16: 10$ & 13 & 13 & 0 \\
\hline $4: 10$ & $4: 20$ & 0 & 0 & 0 & $16: 10$ & $16: 20$ & 14 & 14 & 0 \\
\hline $4: 20$ & $4: 30$ & 2 & 2 & 0 & $16: 20$ & $16: 30$ & 6 & 6 & 0 \\
\hline $4: 30$ & $4: 40$ & 4 & 4 & 0 & $16: 30$ & $16: 40$ & 9 & 9 & 0 \\
\hline $4: 40$ & $4: 50$ & 2 & 2 & 0 & $16: 40$ & $16: 50$ & 12 & 12 & 0 \\
\hline $4: 50$ & $5: 00$ & 9 & 9 & 0 & $16: 50$ & $17: 00$ & 12 & 12 & 0 \\
\hline $5: 00$ & $5: 10$ & 5 & 5 & 0 & $17: 00$ & $17: 10$ & 12 & 12 & 0 \\
\hline $5: 10$ & $5: 20$ & 11 & 11 & 0 & $17: 10$ & $17: 20$ & 9 & 9 & 0 \\
\hline $5: 20$ & $5: 30$ & 11 & 11 & 0 & $17: 20$ & $17: 30$ & 10 & 10 & 0 \\
\hline $5: 30$ & $5: 40$ & 7 & 7 & 0 & $17: 30$ & $17: 40$ & 10 & 10 & 0 \\
\hline $5: 40$ & $5: 50$ & 9 & 9 & 0 & $17: 40$ & $17: 50$ & 9 & 9 & 0 \\
\hline $5: 50$ & $6: 00$ & 10 & 10 & 0 & $17: 50$ & $18: 00$ & 9 & 9 & 0 \\
\hline $6: 00$ & $6: 10$ & 9 & 9 & 0 & $18: 00$ & $18: 10$ & 5 & 5 & 0 \\
\hline $6: 10$ & $6: 20$ & 3 & 3 & 0 & $18: 10$ & $18: 20$ & 8 & 8 & 0 \\
\hline $6: 20$ & $6: 30$ & 3 & 3 & 0 & $18: 20$ & $18: 30$ & 8 & 8 & 0 \\
\hline $6: 30$ & $6: 40$ & 6 & 6 & 0 & $18: 30$ & $18: 40$ & 5 & 5 & 0 \\
\hline $6: 40$ & $6: 50$ & 6 & 6 & 0 & $18: 40$ & $18: 50$ & 4 & 4 & 0 \\
\hline $6: 50$ & $7: 00$ & 6 & 6 & 0 & $18: 50$ & $19: 00$ & 10 & 10 & 0 \\
\hline $7: 00$ & $7: 10$ & 9 & 9 & 0 & $19: 00$ & $19: 10$ & 12 & 12 & 0 \\
\hline $7: 10$ & $7: 20$ & 6 & 6 & 0 & $19: 10$ & $19: 20$ & 16 & 16 & 0 \\
\hline $7: 20$ & $7: 30$ & 6 & 6 & 0 & $19: 20$ & $19: 30$ & 22 & 22 & 0 \\
\hline $7: 30$ & $7: 40$ & 6 & 6 & 0 & $19: 30$ & $19: 40$ & 15 & 15 & 0 \\
\hline $7: 40$ & $7: 50$ & 8 & 8 & 0 & $19: 40$ & $19: 50$ & 14 & 14 & 0 \\
\hline $7: 50$ & $8: 00$ & 11 & 11 & 0 & $19: 50$ & $20: 00$ & 12 & 12 & 0 \\
\hline $8: 00$ & $8: 10$ & 19 & 19 & 0 & $20: 00$ & $20: 10$ & 13 & 13 & 0 \\
\hline $8: 10$ & $8: 20$ & 6 & 6 & 0 & $20: 10$ & $20: 20$ & 6 & 6 & 0 \\
\hline $8: 20$ & $8: 30$ & 10 & 10 & 0 & $20: 20$ & $20: 30$ & 11 & 11 & 0 \\
\hline $8: 30$ & $8: 40$ & 8 & 8 & 0 & $20: 30$ & $20: 40$ & 8 & 9 & -1 \\
\hline $8: 40$ & $8: 50$ & 7 & 7 & 0 & $20: 40$ & $20: 50$ & 2 & 2 & 0 \\
\hline $8: 50$ & $9: 00$ & 7 & 7 & 0 & $20: 50$ & $21: 00$ & 1 & 1 & 0 \\
\hline 9:00 & $9: 10$ & 8 & 8 & 0 & $21: 00$ & $21: 10$ & 2 & 2 & 0 \\
\hline $9: 10$ & $9: 20$ & 6 & 7 & -1 & $21: 10$ & $21: 20$ & 4 & 4 & 0 \\
\hline $9: 20$ & $9: 30$ & 6 & 6 & 0 & $21: 20$ & $21: 30$ & 5 & 5 & 0 \\
\hline 9:30 & $9: 40$ & 6 & 6 & 0 & $21: 30$ & $21: 40$ & 3 & 3 & 0 \\
\hline $9: 40$ & $9: 50$ & 5 & 5 & 0 & $21: 40$ & $21: 50$ & 4 & 4 & 0 \\
\hline 9:50 & $10: 00$ & 2 & 2 & 0 & $21: 50$ & $22: 00$ & 4 & 4 & 0 \\
\hline $10: 00$ & $10: 10$ & 6 & 6 & 0 & $22: 00$ & $22: 10$ & 4 & 4 & 0 \\
\hline $10: 10$ & $10: 20$ & 6 & 6 & 0 & $22: 10$ & $22: 20$ & 3 & 3 & 0 \\
\hline $10: 20$ & $10: 30$ & 4 & 4 & 0 & $22: 20$ & $22: 30$ & 1 & 1 & 0 \\
\hline $10: 30$ & $10: 40$ & 4 & 4 & 0 & $22: 30$ & $22: 40$ & 4 & 4 & 0 \\
\hline $10: 40$ & $10: 50$ & 5 & 5 & 0 & $22: 40$ & $22: 50$ & 3 & 3 & 0 \\
\hline $10: 50$ & $11: 00$ & 11 & 11 & 0 & $22: 50$ & $23: 00$ & 2 & 2 & 0 \\
\hline $11: 00$ & $11: 10$ & 8 & 8 & 0 & $23: 00$ & $23: 10$ & 1 & 1 & 0 \\
\hline $11: 10$ & $11: 20$ & 7 & 7 & 0 & $23: 10$ & $23: 20$ & 0 & 0 & 0 \\
\hline $11: 20$ & $11: 30$ & 6 & 6 & 0 & $23: 20$ & $23: 30$ & 0 & 0 & 0 \\
\hline $11: 30$ & $11: 40$ & 6 & 6 & 0 & $23: 30$ & $23: 40$ & 0 & 0 & 0 \\
\hline $11: 40$ & $11: 50$ & 8 & 8 & 0 & $23: 40$ & $23: 50$ & 0 & 0 & 0 \\
\hline $11: 50$ & $12: 00$ & 6 & 7 & -1 & $23: 50$ & $0: 00$ & 0 & 0 & 0 \\
\hline
\end{tabular}




\subsection{Sector Entry Count}

Algorithms in this thesis for sector occupancy count, and sector entry count, are based on the same input data (EFD file). Reasons for discrepancy between count in NEST and in this thesis are due to a limitation in the thesis algorithm. Table 6.4 shows the count comparison for sector Koksy High. There are 2 flights counted extra by the thesis algorithm. These are flights AA94114508 and AA94127378. In both cases the EFD file indicated that they had actually entered the sector but they have never been ATC activated i.e. the flight has never departed. This is a limitation of the algorithm and it is computationally difficult to recognize such occurrences of 'false' sector entry. 
Table 6.4: Actual sector entry count comparison between NEST and thesis algorithm. Sector Koksy High

\begin{tabular}{|c|c|c|c|c|}
\hline Start bin & End bin & Thesis & NEST & $\begin{array}{l}\text { Thesis } \\
\text { - NEST }\end{array}$ \\
\hline $0: 00$ & $1: 00$ & 13 & 13 & 0 \\
\hline $0: 20$ & $1: 20$ & 7 & 7 & 0 \\
\hline $0: 40$ & $1: 40$ & 4 & 4 & 0 \\
\hline $1: 00$ & $2: 00$ & 4 & 4 & 0 \\
\hline $1: 20$ & $2: 20$ & 6 & 6 & 0 \\
\hline $1: 40$ & $2: 40$ & 6 & 6 & 0 \\
\hline $2: 00$ & $3: 00$ & 5 & 5 & 0 \\
\hline $2: 20$ & $3: 20$ & 6 & 6 & 0 \\
\hline $2: 40$ & $3: 40$ & 7 & 7 & 0 \\
\hline $3: 00$ & $4: 00$ & 8 & 8 & 0 \\
\hline $3: 20$ & $4: 20$ & 4 & 4 & 0 \\
\hline $3: 40$ & $4: 40$ & 7 & 7 & 0 \\
\hline $4: 00$ & $5: 00$ & 9 & 9 & 0 \\
\hline $4: 20$ & $5: 20$ & 15 & 15 & 0 \\
\hline $4: 40$ & $5: 40$ & 22 & 22 & 0 \\
\hline $5: 00$ & $6: 00$ & 31 & 31 & 0 \\
\hline $5: 20$ & $6: 20$ & 39 & 39 & 0 \\
\hline $5: 40$ & $6: 40$ & 49 & 49 & 0 \\
\hline $6: 00$ & $7: 00$ & 53 & 53 & 0 \\
\hline $6: 20$ & $7: 20$ & 46 & 46 & 0 \\
\hline $6: 40$ & $7: 40$ & 30 & 30 & 0 \\
\hline $7: 00$ & $8: 00$ & 22 & 22 & 0 \\
\hline $7: 20$ & $8: 20$ & 27 & 27 & 0 \\
\hline $7: 40$ & $8: 40$ & 35 & 35 & 0 \\
\hline $8: 00$ & $9: 00$ & 40 & 40 & 0 \\
\hline $8: 20$ & $9: 20$ & 40 & 40 & 0 \\
\hline $8: 40$ & $9: 40$ & 39 & 39 & 0 \\
\hline 9:00 & $10: 00$ & 36 & 36 & 0 \\
\hline $9: 20$ & $10: 20$ & 32 & 32 & 0 \\
\hline $9: 40$ & $10: 40$ & 33 & 33 & 0 \\
\hline $10: 00$ & $11: 00$ & 43 & 43 & 0 \\
\hline $10: 20$ & $11: 20$ & 50 & 50 & 0 \\
\hline $10: 40$ & $11: 40$ & 50 & 50 & 0 \\
\hline $11: 00$ & $12: 00$ & 43 & 42 & 1 \\
\hline $11: 20$ & $12: 20$ & 42 & 41 & 1 \\
\hline $11: 40$ & $12: 40$ & 43 & 42 & 1 \\
\hline $12: 00$ & $13: 00$ & 44 & 44 & 0 \\
\hline $12: 20$ & $13: 20$ & 48 & 48 & 0 \\
\hline $12: 40$ & $13: 40$ & 46 & 46 & 0 \\
\hline $13: 00$ & $14: 00$ & 49 & 49 & 0 \\
\hline $13: 20$ & $14: 20$ & 43 & 43 & 0 \\
\hline $13: 40$ & $14: 40$ & 45 & 45 & 0 \\
\hline $14: 00$ & $15: 00$ & 41 & 41 & 0 \\
\hline $14: 20$ & $15: 20$ & 46 & 46 & 0 \\
\hline $14: 40$ & $15: 40$ & 46 & 45 & 1 \\
\hline $15: 00$ & $16: 00$ & 40 & 39 & 1 \\
\hline $15: 20$ & $16: 20$ & 38 & 37 & 1 \\
\hline $15: 40$ & $16: 40$ & 38 & 38 & 0 \\
\hline $16: 00$ & $17: 00$ & 41 & 41 & 0 \\
\hline $16: 20$ & $17: 20$ & 41 & 41 & 0 \\
\hline $16: 40$ & $17: 40$ & 35 & 35 & 0 \\
\hline $17: 00$ & $18: 00$ & 35 & 35 & 0 \\
\hline $17: 20$ & $18: 20$ & 40 & 40 & 0 \\
\hline $17: 40$ & $18: 40$ & 42 & 42 & 0 \\
\hline $18: 00$ & $19: 00$ & 48 & 48 & 0 \\
\hline $18: 20$ & $19: 20$ & 34 & 34 & 0 \\
\hline $18: 40$ & $19: 40$ & 31 & 31 & 0 \\
\hline 19:00 & $20: 00$ & 23 & 23 & 0 \\
\hline $19: 20$ & $20: 20$ & 30 & 30 & 0 \\
\hline $19: 40$ & $20: 40$ & 33 & 33 & 0 \\
\hline $20: 00$ & $21: 00$ & 34 & 34 & 0 \\
\hline $20: 20$ & $21: 20$ & 39 & 39 & 0 \\
\hline $20: 40$ & $21: 40$ & 35 & 35 & 0 \\
\hline $21: 00$ & $22: 00$ & 33 & 33 & 0 \\
\hline $21: 20$ & $22: 20$ & 22 & 22 & 0 \\
\hline $21: 40$ & $22: 40$ & 22 & 22 & 0 \\
\hline $22: 00$ & $23: 00$ & 17 & 17 & 0 \\
\hline $22: 20$ & $23: 20$ & 13 & 13 & 0 \\
\hline $22: 40$ & $23: 40$ & 9 & 9 & 0 \\
\hline $23: 00$ & $6 / 29 / 130: 00$ & 11 & 11 & 0 \\
\hline $23: 20$ & $6 / 29 / 13 \quad 0: 20$ & 8 & 8 & 0 \\
\hline $23: 40$ & $6 / 29 / 130: 40$ & 5 & 5 & 0 \\
\hline
\end{tabular}




\subsection{Conclusions}

This chapter makes a comparison of actual traffic count obtained by the algorithm from this thesis, and by the software tool NEST, for 2 sectors. The difference in count can be considered small; 9 additional occupancy count flights in NEST, and 2 additional entry count flights in the thesis algorithm. It can be said that that the actual count obtained by the thesis algorithm accurately reflects the real count. 


\section{Chapter 7}

\section{Arrival Time Predictability}

This chapter discusses predictability of airport arrival times. A differentiation is made between flights that have 1, 2, and 3 hours flight time from origin to destination airport. For these flights the mean prediction error, standard deviation prediction error, and accumulated flights plans are given, all as function of look-ahead time to take-off. This is done in order to quantify uncertainty along the complete flight time horizon.

\subsection{Population Sample}

For the analysis done in this chapter some flights are excluded. The following itemization show the exclusions made, where $\mathrm{A} \subset \mathrm{B}$ means that $\mathrm{A}$ is part of the bigger group $\mathrm{B}$.

- Flights departing from airports in ECAC member states (figure 4.1a) $\subset$ Flights being present sometime during the day within the extended ECAC area (figure $4.1 \mathrm{~b})$.

- Flights that are scheduled air transport $\subset$ Flights departing from airports from ECAC member states.

- Flights that are not cancelled $\subset$ Flights that are scheduled air transport.

- Flights that were predicted to enter and did enter $\subset$ Flights that are not cancelled.

Flights departing from airports in ECAC member states are analyzed because recorded departure times for these flights are accurate. It occurs that departure times for flights are recorded from airports outside ECAC states which are not in accordance with the real departure times. Furthermore military and general aviation flights are excluded because previous research indicates that they hardly adhere to flight plans, resulting in large prediction error [11]. Non-entered flights are excluded, because it is unrealistic to calculate a prediction error if the flight never entered the sector. 
Table 7.1: Number of daily flights with flight time 1, 2, and 3 hours.

\begin{tabular}{l|lll} 
Day & $\begin{array}{l}\text { Flights with } \\
\text { flight time } \approx\end{array}$ & $\begin{array}{l}\text { Flights with } \\
\text { flight time } \approx \\
\text { 2h }\end{array}$ & $\begin{array}{l}\text { Flights with } \\
\text { flight time } \approx \\
3 \mathrm{~h}\end{array}$ \\
\hline 27 June 2013 & 3996 & 1580 & 507 \\
28 June 2013 & 4062 & 1621 & 535 \\
29 June 2013 & 2782 & 1700 & 626 \\
30 June 2013 & 3255 & 1628 & 517 \\
4 March 2014 & 3513 & 993 & 262 \\
5 March 2014 & 3514 & 1045 & 259 \\
6 March 2014 & 3603 & 1036 & 273 \\
7 March 2014 & 3640 & 1152 & 300 \\
8 February 2014 & 2161 & 971 & 326 \\
1 February 2014 & 2107 & 936 & 299 \\
18 January 2014 & 2040 & 948 & 292 \\
\hline Total & 34673 & 13610 & 3896
\end{tabular}

To obtain a statistical significant sample size, 11 days were analyzed (table 7.1). The chosen days consists of small, average, and large daily traffic levels. For the time period between March 2013 and March 2014, the 28th of June 2013 has the largest number of flights; the 7th of March 2014 has approximately the average number of daily flights, and the days in February have the smallest amount of daily traffic. Table 7.1 shows the number of flights for the given days after the exclusions made. Take note that in table 7.1 , flight time $\approx 1$ hour means that flights are included that have a flight time of 1 hour \pm 15 minutes. The same holds for flight time 2 and 3 hours.

\subsection{Methodology}

As discussed in previous chapters, mean and standard deviation are used to describe variation of the prediction error.

Mean arrival time prediction error is calculated by fitting a curve thought the arrival time prediction error versus look-ahead data points as described in section 5.2.1.

Methodology to obtain standard deviation of the arrival time prediction error (section 7.5 ) is explained by means of the individual flight example. Assume that sector entry time becomes airport arrival time in table 1.1. The first prediction after take-off is at 6 minutes after take-off, the "arrival" time prediction error is 7 minutes. This is indicated in figure 7.1a as the cyan dot. Now obtaining prediction error for all flights in this 10 minute slice yields figure 7.1b. The standard deviation for all errors inside this slice is $\sim 7$ minutes. Plotting this error, and the error for other slices in the same manner yields figure 7.3 . 


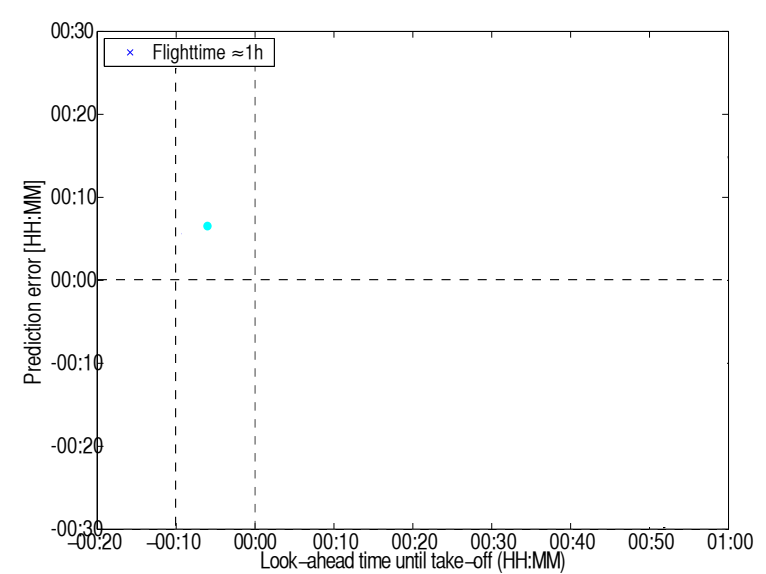

(a) Single arrival time prediction error from example flight.

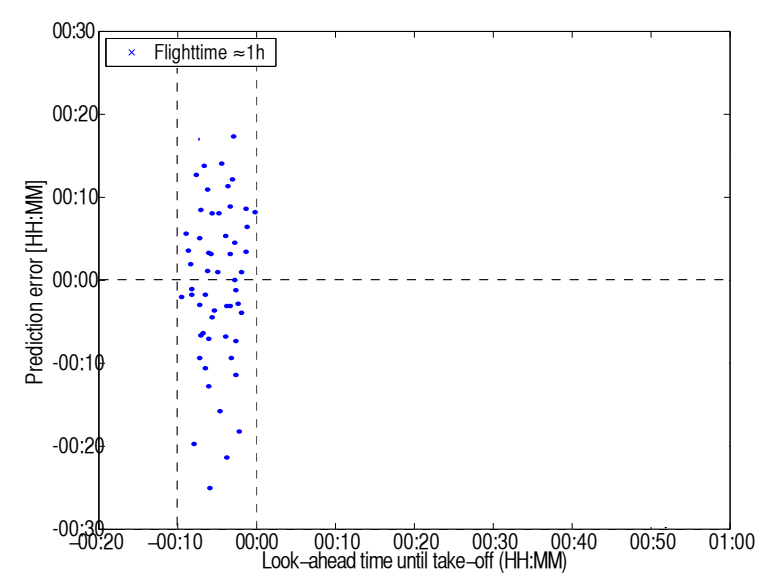

(b) Multiple arrival time prediction errors.

Figure 7.1: Example of arrival time prediction error for the 10 minute slice after take-off.

\subsection{Confidence bounds}

Figure 7.4 indicates the standard deviation of the arrival time prediction error, for flights with 1 hour flight time. The standard deviation is calculated in slices of 10 minutes, but due to a lack of data availability the slice width is larger for longer look-ahead times than 2.5 hours before take-off. Hence the slices are 30 minutes. Confidence bounds are calculated for the first 10 minute slice after take-off, and for the slice with the longest look-ahead time before take-off, which is 355 minutes before take-off. 95\% confidence bounds for the first slice are calculated using equation 3.8 :

$$
\left(06: 10-1.96 \frac{06: 12}{\sqrt{187007}}, 06: 10+1.96 \frac{06: 12}{\sqrt{187007}}\right)=(06: 06,06: 14) \quad \text { MM:SS }
$$

And the upper and lower confidence bounds for the last slice:

$$
\left(23: 54-1.96 \frac{24: 27}{\sqrt{16473}}, 23: 54+1.96 \frac{24: 27}{\sqrt{16473}}\right)=(23: 09,24: 39) \quad \text { MM:SS }
$$

For both look-aheads the interval is fairly small, which means that figure 7.4 is a good representation for the true value of the standard deviation.

\subsection{Mean Arrival Time Prediction Error}

Section 5.2.1 explains the methodology of curve fitting through prediction error data points. This is done in similar fashion in this section, in order to obtain mean arrival time prediction error. This is done for arrival time prediction error (figure 7.2a) and absolute arrival time prediction error $(7.2 \mathrm{~b})$. 
Take note that look-ahead time is not anymore until sector entry (as in previous chapters), but until take-off. This allows for quantification of uncertainty along the flight time horizon. A negative look-ahead time until take-off means time after take-off.

The steepest reduction of the mean arrival time prediction error occurs at descent, at taxi, and at the slot allocation process. A possible explanation is that standard terminal arrival route (STAR) and taxi time predictions are inaccurate. Prediction error is larger for flights with longer flight times. The reason for this is unknown and further investigation is recommended. A possible explanation is that this could be due to smaller data sample for flights with longer flight times.

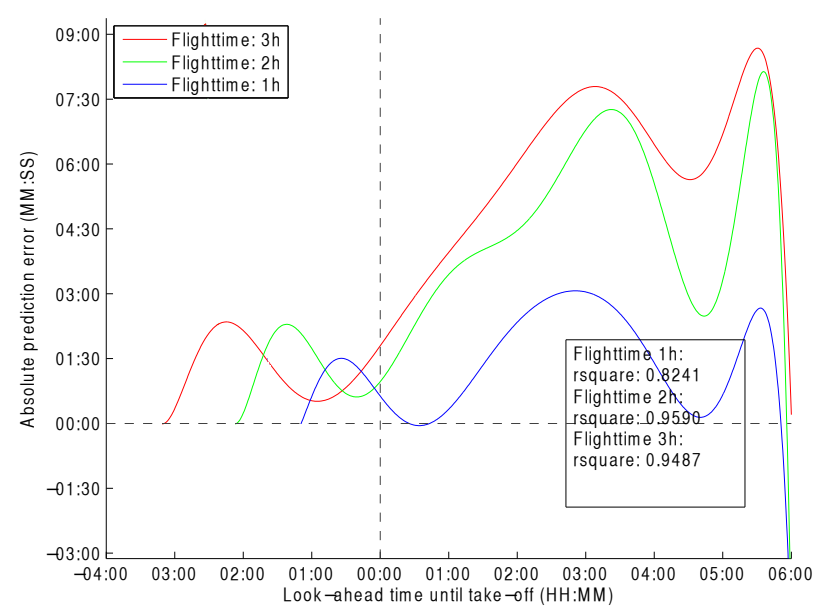

(a) Non-absolute prediction error.

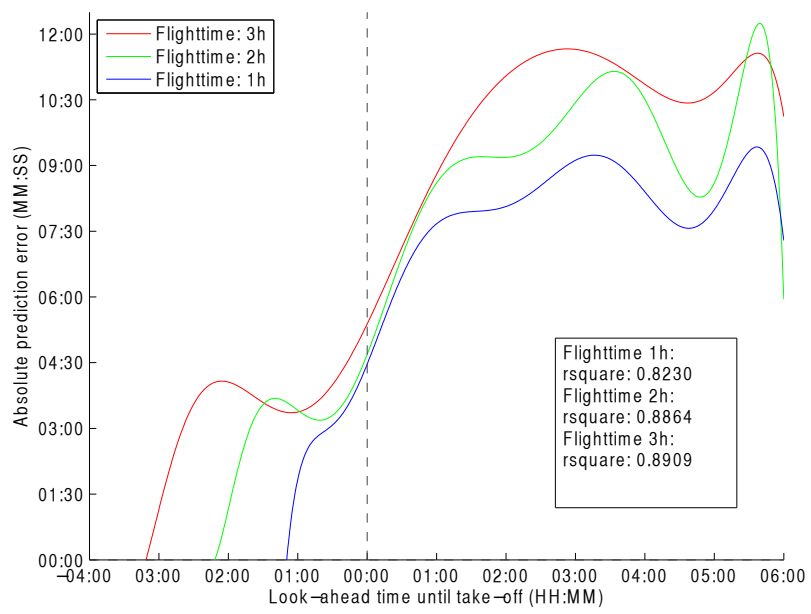

(b) Absolute prediction error

Figure 7.2: Mean arrival time prediction error for flights with approximately 1,2, and 3 hour flight time.

\subsection{Standard Deviation Arrival Time Prediction Error}

Turning from mean to standard deviation of arrival time prediction error. Plotting standard deviation of arrival time prediction error as described in section 7.2 yields figure 7.3. Figure 7.4 quantifies the standard deviation error reduction for different flight phases. Hereby the following flight phases are identified:

- $\Delta$ 1: From start slot allocation until end slot allocation.

- $\Delta$ 2: From end slot allocation until off block time. In this time interval pre-flight preparation is executed (baggage handling, turnaround process, etc.)

- $\Delta$ 3: From off block time until take-off time. This is the taxi phase.

- $\Delta$ 4: From take-off to top of descent. In this time interval the flight is following standard instrument departure. 
- $\Delta$ 5: From top of descent until landing. In this time interval the flight is following the standard terminal arrival route.

By appointing time intervals in figure 7.4, the following assumptions are made; the slot allocation process ends at 2 hours before off block time, taxi time takes 20 minutes, top of descend is 30 minutes before landing.

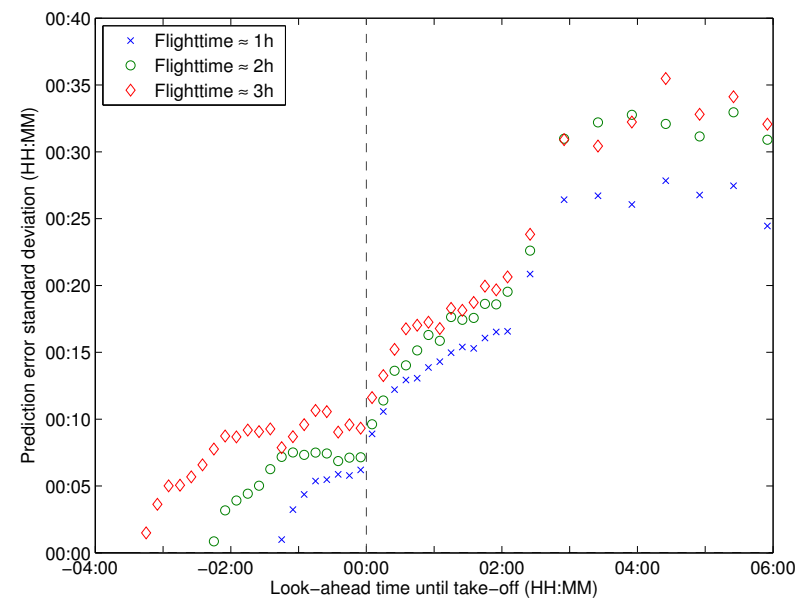

Figure 7.3: Standard deviation of arrival time prediction error for flights with approximately 1,2 , and 3 hours flight time.

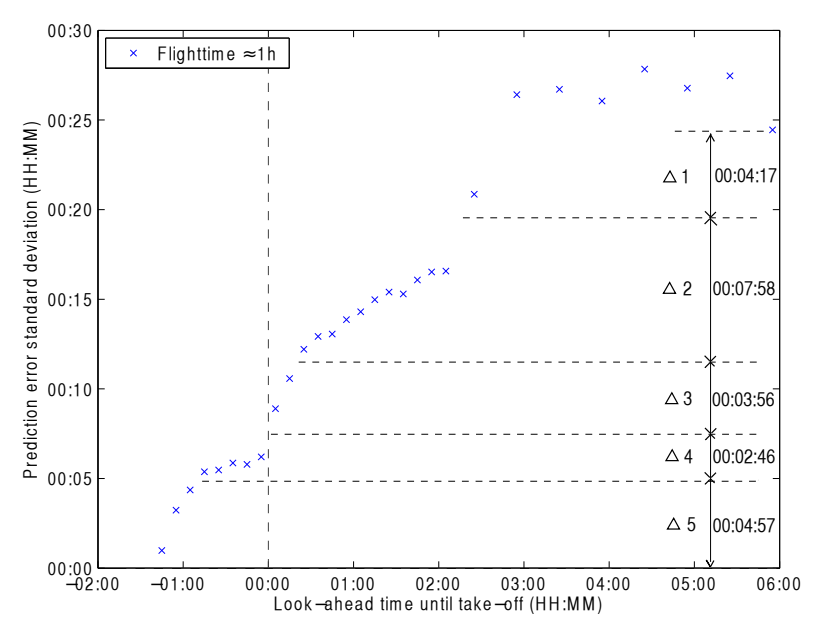

Figure 7.4: Standard deviation of prediction error for flights with approximately 1 hour flight time, with quantification of error reductions at several time instances.

Possible explanations for an uncertainty reduction at the delta's are:

- $\Delta$ 1: If a slot is allocated for a filed flight plan, the trajectory prediction is more accurate.

- $\Delta$ 2: Pre-flight preparation takes place in this interval e.g. bagage handling, aircraft turnaround, etc. This process causes prediction uncertainty.

- $\Delta$ 3: Taxi time predictions are inaccurate. Every airport assumes an average taxi time period for all flights. The real taxi time differs from flight to flight, the discrepancy between assumed and real taxi time causes prediction uncertainty.

- $\Delta$ 4: Difference between the predicted standard instrument departure route and the actual standard instrument departure route could be a prediction error source. .

- $\Delta$ 5: Difference between the predicted and actual standard terminal arrival route could be a prediction error source.

Figure 7.5 compares the standard deviation for flights for different traffic levels. As expected, standard deviation is larger for busy traffic than for normal traffic. This difference is quantified for 2 time instances. Larger standard deviation on calm days compared to normal days in this case can be explained due to the difference in sample size. As can be seen from table 7.1 the sample size for the normal days is double the sample size of the calm days sample size. Sample size for the set of normal and busy days is almost the same. 


\subsection{Accumulated Flight Plans}

To explain the dispersion of standard deviation at longer look-ahead times in figure 7.3, accumulated flight plans as function of look-ahead is plotted in figure 7.6. At 6 hours before take-off, $80 \%$ of the flight plans have been filed, which causes the standard deviation dispersion at longer look-ahead.

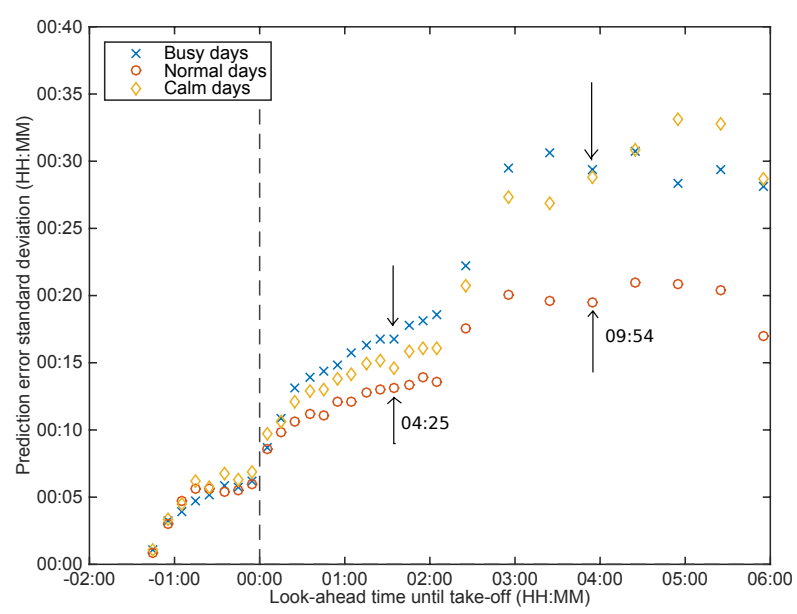

Figure 7.5: Standard deviation of arrival time prediction error for flights with approximately 1 hour flight time for different traffic levels.

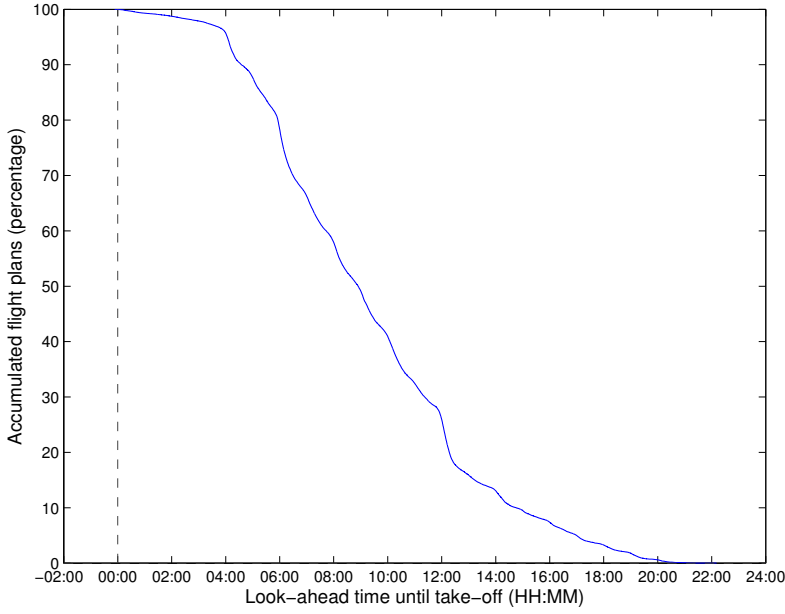

Figure 7.6: Percentage of accumulated flight plans before take-off, for flights with 1 hour flight time..

\subsection{Conclusions}

This section highlights the main points of this chapter on arrival time predictability:

- Flight phases along the flight time horizon that show relatively poor prediction accuracy are; the slot allocation process, the taxi phase, and the descent phase.

- Prediction uncertainty in the slot allocation process can be explained by the discrepancy between initially filed flight plans by Aircraft Operators, and the actually allocated departure slots by the ETFMS. The contribution of this uncertainty is $18 \%$ of the total uncertainty at 6 hours before take-off.

- Prediction uncertainty in the taxi phase can be explained by discrepancy between the average predicted taxi-time for an airport which holds for all flights, and the real taxi time that differs from flight to flight. The contribution of this uncertainty is $11 \%$ of the total uncertainty at 6 hours before take-off.

- Prediction uncertainty in the descent phase can be explained by discrepancy between the predicted and actual standard terminal arrival route. The contribution of this uncertainty is $21 \%$ of the total uncertainty at 6 hours before take-off. 


\section{Chapter 8}

\section{Sensitivity Analysis}

This chapter aims to quantify sector demand predictability changes, in case the pilot is able to stick better to the planned take-off time. This "better take-off time adherence" or "improved departure time prediction" is henceforth called "reduced departure time prediction error". Prior to commencing discussion of sensitivity analysis results, section 8.1 discusses the reason for choosing the method of historical flight data alteration. Section 8.2 describes the procedure of such alteration using the example of the British Airways flight. Changes of predictability are shown by comparing obtained predictability figures from previous chapters with obtained curves from the sensitivity analysis. These predictability figures are on sector entry time, sector occupancy count, airport arrival time.

\subsection{Methodology}

\section{Why departure time prediction error reduction?}

SESAR aims to improve predictability with trajectory based operations. Assumed is that Airspace Users better adhere to planned trajectories, and therefore the effect of improved predictability is evaluated.

Factors that have a high influence on sector demand predictability are departure time prediction error, ATC procedures (level capping, direct routing, etc.), and ATFCM regulations [10]. Literature and experts indicate that the factor "departure time prediction error", has the most influence on sector demand predictability. Sensitivity analysis on ATC procedures is difficult as data is hardly available, sensitivity analysis of ATFCM regulations only affects approximately $10 \%$ of the flights. For these reasons sensitivity analysis on departure time prediction error is chosen. 


\section{Why historical data alteration over fast time simulation?}

It is possible to reduce departure time prediction error in state-of-the-art fast time simulation software e.g. AirTOp. Another option is to change prediction times of flights included in the preceding statistical analysis, This is henceforth called "historical data alteration". Table 8.1 discusses pro's and con's of both tools. It is chosen to alter historical data, because results from previous chapters can be easily compared.

Table 8.1: Trade-off between historical data alteration \& fast time simulation.

\begin{tabular}{|c|c|c|}
\hline Tool & Pro & Con \\
\hline $\begin{array}{l}\text { Historical } \\
\text { data } \\
\text { alteration }\end{array}$ & $\begin{array}{l}\text { Algorithms from previous statistical } \\
\text { analysis can be easily altered and } \\
\text { compared. }\end{array}$ & $\begin{array}{l}\text { Flight separation minima that might be } \\
\text { exceeded due to altered times are not } \\
\text { included. }\end{array}$ \\
\hline $\begin{array}{l}\text { Fast } \\
\text { time } \\
\text { simulation }\end{array}$ & $\begin{array}{l}\text { Aggregated effect of factors dependent on } \\
\text { changed predictability is accounted for } \\
\text { e.g factors as ATC procedures or ATFCM } \\
\text { regulations }\end{array}$ & $\begin{array}{l}\text { Only initial and actual flight plans } \\
\text { are able to be processed as input. It is not } \\
\text { possible to obtain predictions between } \\
\text { initial and actual flight trajectories. }\end{array}$ \\
\hline
\end{tabular}

\section{How much prediction error reduction is realistic?}

The sensitivity analysis is done by a percentage departure time prediction error reduction. The range of percentages are from 0 to $50 \%$. $0 \%$ is no alteration, and $50 \%$ is the most realistically obtainable improvement stated by expert opinion. In sections 8.2 an example of a $50 \%$ error reduction is given. The reference times at which the errors are reduced are at off block time (AOBT) and at 1 hour before off block time (AOBT-1h.). These instances are chosen because improvements at those times are most realistically obtainable. If the first available prediction for a non-regulated flight is later than AOBT or AOBT-1h, no alteration is made for that flight. For regulated flights, departure time predictions are improved with respect to the last slot issue time before take-off.

\subsection{Individual Flight Example}

For the individual flight example, table 8.2a gives unaltered predictions as stated in table 1.1. Table $8.2 \mathrm{~b}$ shows $50 \%$ departure time prediction error reduction. The error is reduced with respect to the reference time: 1 hour before off block (AOBT-1h).

To obtain times in table $8.2 \mathrm{~b}$ from table $8.2 \mathrm{a}$, the following steps are taken. First departure time prediction error ( $\epsilon$.ATOT) is calculated for every prediction i (row):

$$
\epsilon \cdot A T O T_{i}=E T O T_{i}-E T O T \text { at } A O B T-1 \mathrm{~h}
$$

where $\mathrm{ETOT}_{i}$ is predicted departure time (this could also be actual departure time for a "prediction" after departure). The time shift per prediction is obtained by:

$$
\text { Timeshift }_{i}=\epsilon \cdot A T O T_{i} * \text { Percentage error reduction }
$$




\begin{tabular}{|c|c|c|c|c|c|}
\hline & $\begin{array}{l}\text { Look- } \\
\text { ahead } \\
\text { to } \\
\text { sector } \\
\text { entry }\end{array}$ & $\begin{array}{l}\text { Sector } \\
\text { entry } \\
\text { time } \\
\text { prediction } \\
\text { error }\end{array}$ & $\begin{array}{l}\text { Time- } \\
\text { stamp }\end{array}$ & $\begin{array}{l}\text { Take- } \\
\text { off } \\
\text { time }\end{array}$ & $\begin{array}{l}\text { Sector } \\
\text { entry } \\
\text { time }\end{array}$ \\
\hline \multirow[t]{3}{*}{ Off block-1h $\rightarrow$} & $01: 54$ & $00: 14$ & $10: 10$ & $11: 20$ & $11: 50$ \\
\hline & 01:09 & $00: 14$ & $10: 55$ & $11: 20$ & $11: 50$ \\
\hline & $00: 56$ & $00: 12$ & 11:08 & $11: 20$ & $11: 52$ \\
\hline \multirow[t]{3}{*}{ Off Block $\rightarrow$} & $-\ldots$ & $-\ldots$ & $-\cdots$ & $-\ldots$ & $-\ldots$ \\
\hline & $00: 46$ & $00: 10$ & $11: 18$ & $11: 22$ & $11: 54$ \\
\hline & $00: 36$ & 00:08 & $11: 28$ & $11: 24$ & $11: 56$ \\
\hline \multirow[t]{6}{*}{ Take-off $\rightarrow$} & $-\ldots$ & - - - - - - & $-\ldots$ & $-\ldots$ & $-\ldots$ \\
\hline & $00: 28$ & 00:07 & $11: 36$ & $11: 30$ & $11: 57$ \\
\hline & $00: 26$ & 00:07 & $11: 38$ & 11:30 & $11: 57$ \\
\hline & $00: 14$ & 00:02 & $11: 50$ & $11: 30$ & $12: 02$ \\
\hline & 00:04 & 00:01 & $12: 00$ & $11: 30$ & $12: 03$ \\
\hline & 00:00 & 00:00 & $12: 04$ & $11: 30$ & $12: 04$ \\
\hline
\end{tabular}

(a) Unaltered predictions.

\begin{tabular}{|c|c|c|c|c|}
\hline $\begin{array}{l}\text { Look- } \\
\text { ahead } \\
\text { to } \\
\text { sector } \\
\text { entry }\end{array}$ & $\begin{array}{l}\text { Sector } \\
\text { entry } \\
\text { time } \\
\text { prediction } \\
\text { error }\end{array}$ & $\begin{array}{l}\text { Time- } \\
\text { stamp }\end{array}$ & $\begin{array}{l}\text { Take- } \\
\text { off } \\
\text { time }\end{array}$ & $\begin{array}{l}\text { Sector } \\
\text { entry } \\
\text { time }\end{array}$ \\
\hline 01:49 & 00:09 & $10: 10$ & $11: 20$ & $11: 50$ \\
\hline 01:04 & 00:09 & $10: 55$ & $11: 20$ & $11: 50$ \\
\hline 00:51 & 00:07 & $11: 08$ & $11: 20$ & $11: 52$ \\
\hline & - - & $-\ldots$ & $-\cdots$ & -- \\
\hline 00:42 & 00:06 & $11: 17$ & $11: 21$ & $11: 53$ \\
\hline 00:33 & 00:05 & $11: 26$ & $11: 22$ & $11: 54$ \\
\hline - - - - - - - & - - - - - - & - - - - - - - & - - - - - - - & $-\ldots$ \\
\hline $00: 28$ & 00:07 & $11: 31$ & $11: 25$ & $11: 52$ \\
\hline $00: 26$ & 00:07 & $11: 33$ & $11: 25$ & $11: 52$ \\
\hline 00:14 & 00:02 & $11: 45$ & $11: 25$ & $11: 57$ \\
\hline 00:04 & 00:01 & $11: 55$ & $11: 25$ & $11: 58$ \\
\hline 00:00 & 00:00 & $11: 59$ & $11: 25$ & $11: 59$ \\
\hline
\end{tabular}

(b) Altered predictions

Table 8.2: Example of altered \& unaltered trajectory predictions for the British Airways flight. 50\% departure time prediction error reduction at 1 hour before off block.

where the error reduction is $50 \%$ in this case. The altered departure time is:

$$
\text { Altered } E T O T_{i}=\text { Unaltered } E T O T_{i}-\text { Timeshift }_{i}
$$

The altered timestamp, and altered sector entry time are calculated by replacing Unaltered $E T O T_{i}$ by unaltered timestamp and unaltered sector entry time, in equation 8.3. Altered look-ahead and prediction error are calculated as explained in section 3.3. Figure 8.1 compares the altered \& unaltered prediction error graphically. From this figure we can see that prediction error after take-off is the same for altered \& unaltered times. This is because predicted entry time and actual entry time are changed (altered) with the same value after take-off, and entry time predictions before take-off are not changed.

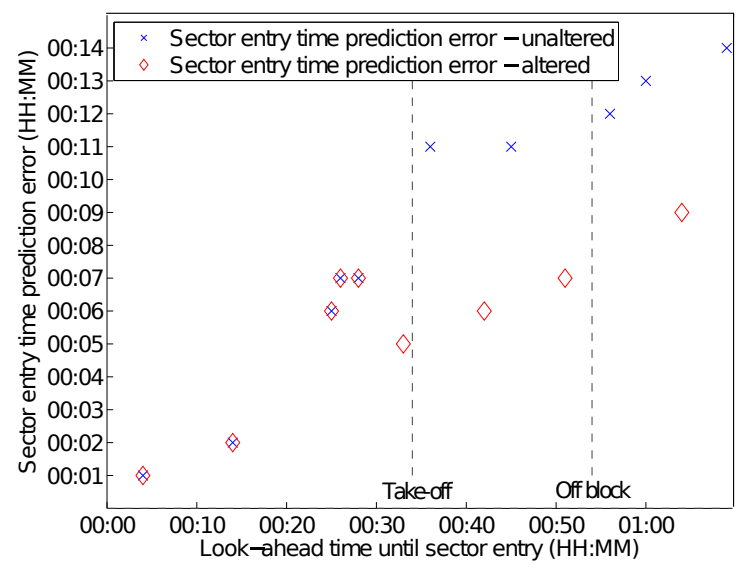

Figure 8.1: Example prediction error for the unaltered and altered individual flight predictions based on table 8.2. 


\subsection{Sector Demand Predictability}

\subsubsection{Sector Entry Time Predictability}

After explanation of the sensitivity analysis method, this section turns to sensitivity of mean sector entry time prediction error. Prediction error is compared for different sectors in figure 8.2. For a look-ahead of 0 to 2 hours mean error curves are insensitive because most flights have departed. Altered and unaltered prediction error are the same for departed flights as discussed in section 8.2. Later than 2 hour look-ahead the sensitivity for mean sector entry time prediction error for the 3 analyzed sectors are fairly similar.

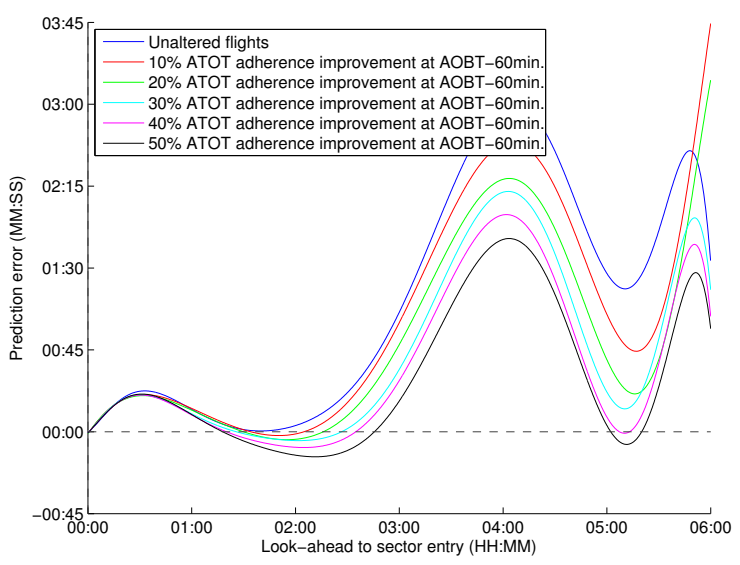

(a) Sector Koksy High.

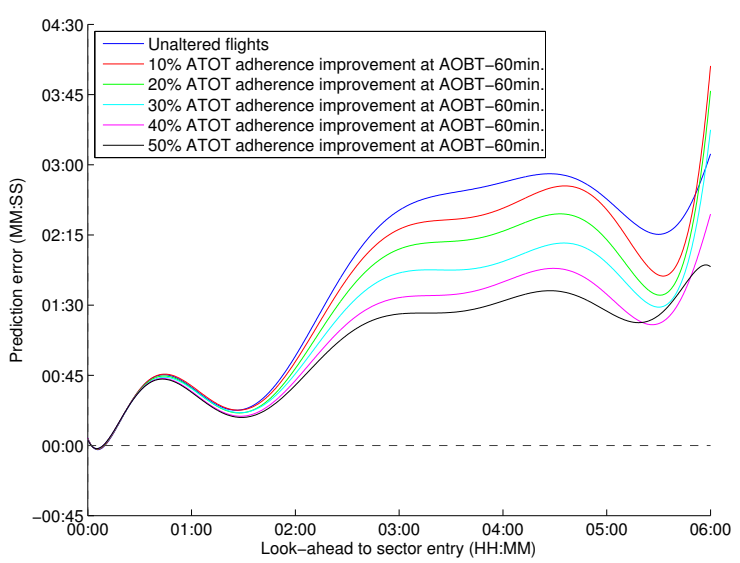

(b) Sector Lux Low.

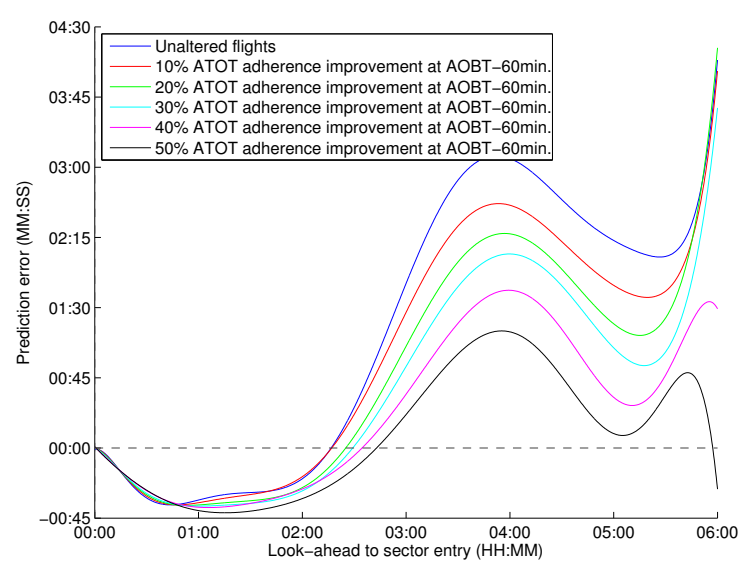

(c) Sector Nicky High.

Figure 8.2: Mean sector entry time prediction error sensitivity for 3 sector, departure time prediction error reduction done at 1 hour before off block.

Sensitivity for mean sector entry time absolute prediction error at off block time, and 1 hour before off block, are similar, indicating that taxi-time predictions are a major source of uncertainty (figure 8.3).

Figures in this section show that it is functional to reduce departure prediction error. If the pilots adheres $50 \%$ better to planned departure times at off block time, an improvement of 
$\sim 50 \%$ for mean non-absolute entry time prediction error, and $\sim 30 \%$ for mean absolute entry time prediction error, can be obtained.

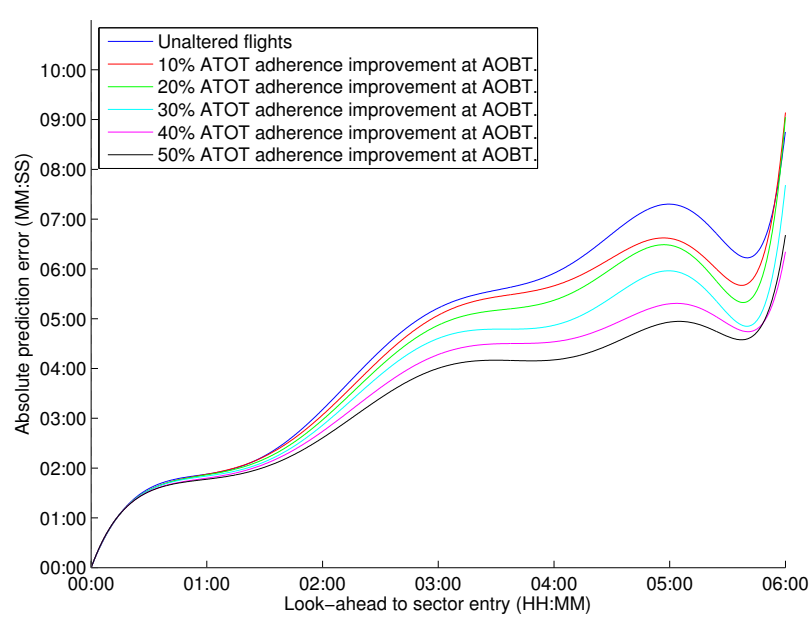

(a) At off block time.

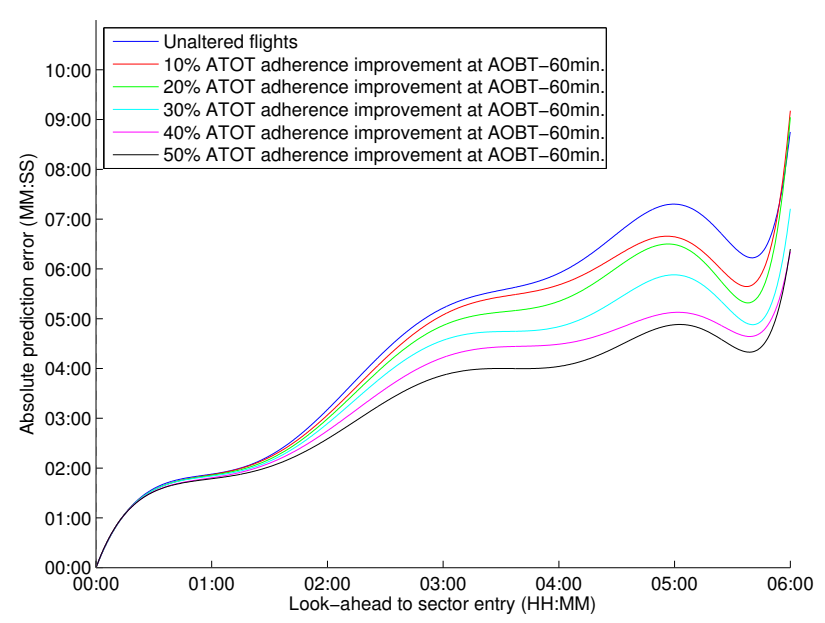

(b) At 1 hour before off block.

Figure 8.3: Mean sector entry time absolute prediction error sensitivity, departure time prediction error reduction done at different times before off block. Sector Koksy High.

\subsubsection{Sector Occupancy Count Predictability}

From entry time sensitivity, now turning to investigation of occupancy count sensitivity. Sensitivity is similar for error reduction done at off block, or 1 hour before off block (figure 8.4). This means that taxi time predictions are a major cause of uncertainty with regard to the time period between take-off and 1 hour before off-block.

One unanticipated finding from figure 8.5 is that reduction in departure time prediction error in some cases results in worse mean sector occupancy count prediction error. The worsening of count prediction error is caused by the imbalance of inflow/outflow flights after a prediction improvement (inflow/outflow flights ratio determines count prediction error). However, both inflow and outflow flights decrease after an error reduction, see table 8.3. These findings are rather disappointing because the used method does not yield realistic sector occupancy counts because the inflow/outflow ratio is unrealistically altered. A possible explanation is that ATC procedures and improved predictability are interdependent, and reduction of departure time prediction error alone does not yield realistic prediction count error. Sensitivity analysis results therefore need to be interpreted with caution. 


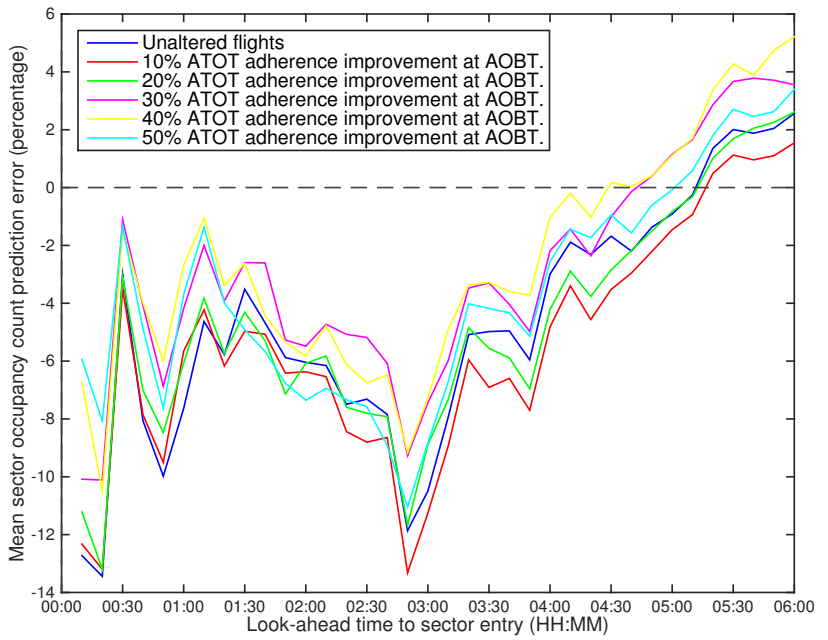

(a) Error reduction at off block time

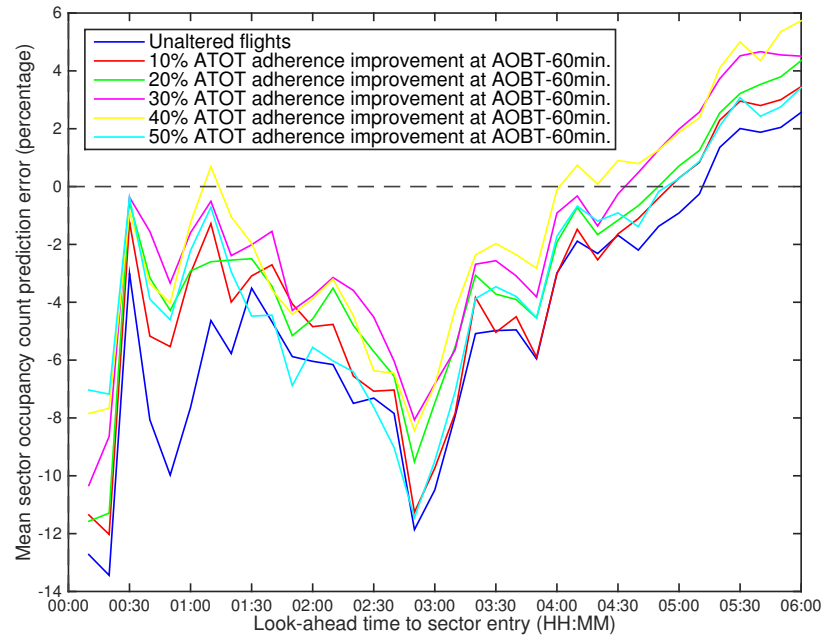

(b) Error reduction at 1 hour before off block

Figure 8.4: Mean sector occupancy count prediction error sensitivity for departure time prediction error reductions at different times before off block. Sector Koksy High.

Table 8.3: Inflow \& outflow flights, for departure time error reduction of 0 to $50 \%$ done at 1 hour before off block. Sector Koksy High. Supplementary to this data is mean count prediction error in figure $8.5 \mathrm{a}$

\begin{tabular}{lllll}
\hline $\begin{array}{l}\text { Departure } \\
\text { time pre- } \\
\text { diction error } \\
\text { reduction }\end{array}$ & $\begin{array}{l}\text { Look- } \\
\text { ahead to } \\
\text { sector } \\
\text { entry } \\
\text { (min) }\end{array}$ & $\begin{array}{l}\text { Inflow } \\
{[\%]}\end{array}$ & $\begin{array}{l}\text { Outflow } \\
{[\%]}\end{array}$ & $\begin{array}{l}\text { Inflow/ } \\
\text { Outflow } \\
\text { ratio }\end{array}$ \\
\hline $0 \%$ & 60 & 25,1 & 28,2 & \\
$10 \%$ & 60 & 26,6 & 26,9 & 0,89 \\
$20 \%$ & 60 & 26 & 26,2 & 0,99 \\
$30 \%$ & 60 & 25,2 & 25,3 & 1,00 \\
$40 \%$ & 60 & 25,5 & 24,9 & 1,02 \\
$50 \%$ & 60 & 24,9 & 24,3 & 1,02 \\
\hline
\end{tabular}




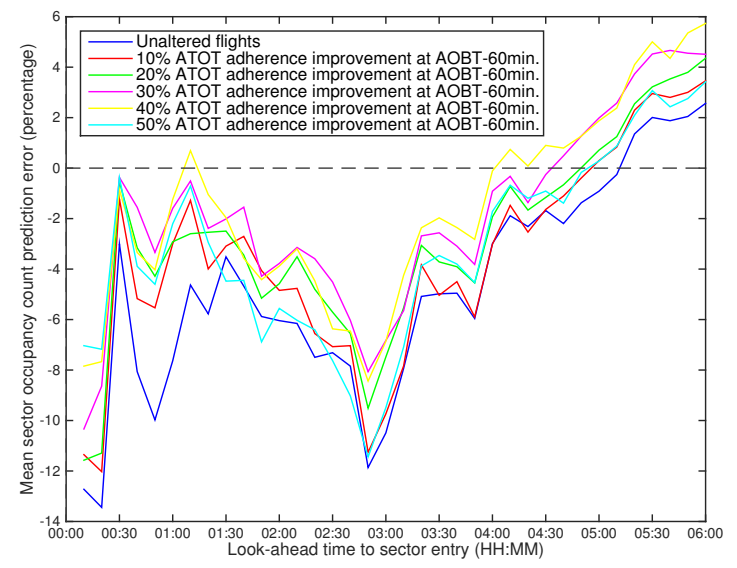

(a) Mean, sector Koksy High

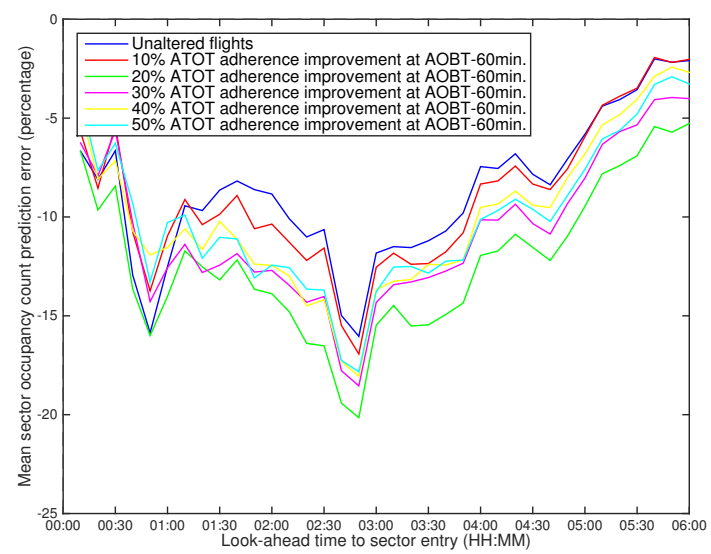

(c) Mean, sector Nicky High

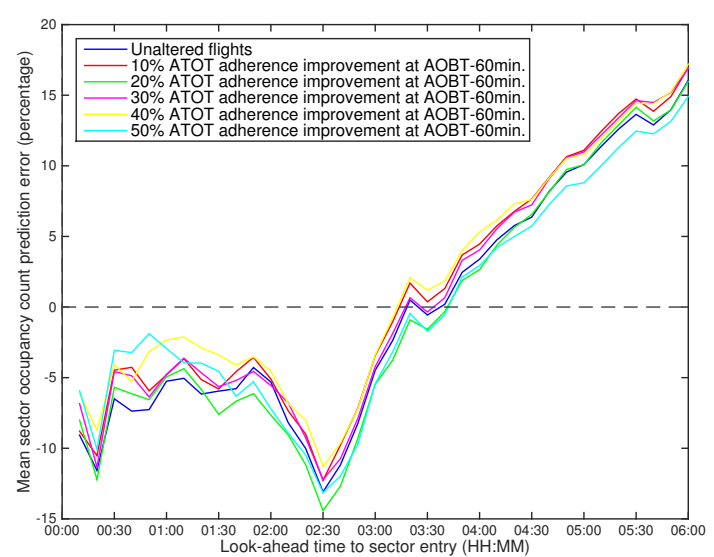

(e) Mean, sector Lux Low

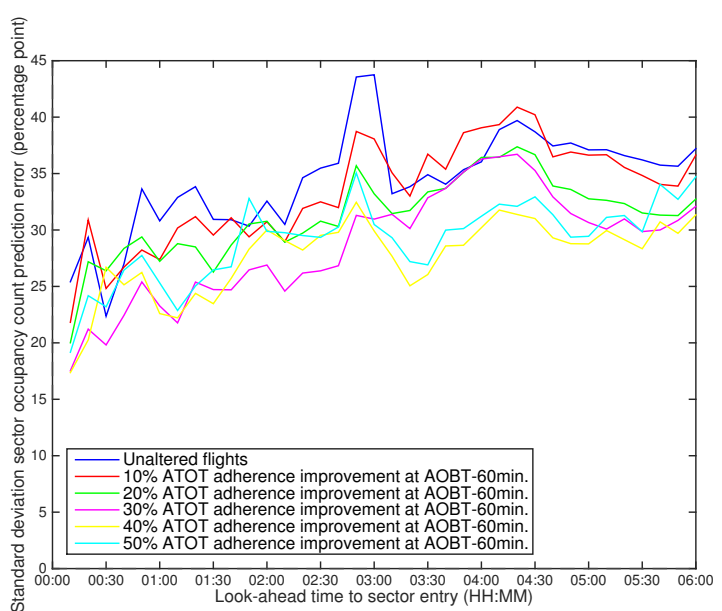

(b) Standard deviation, sector Koksy High

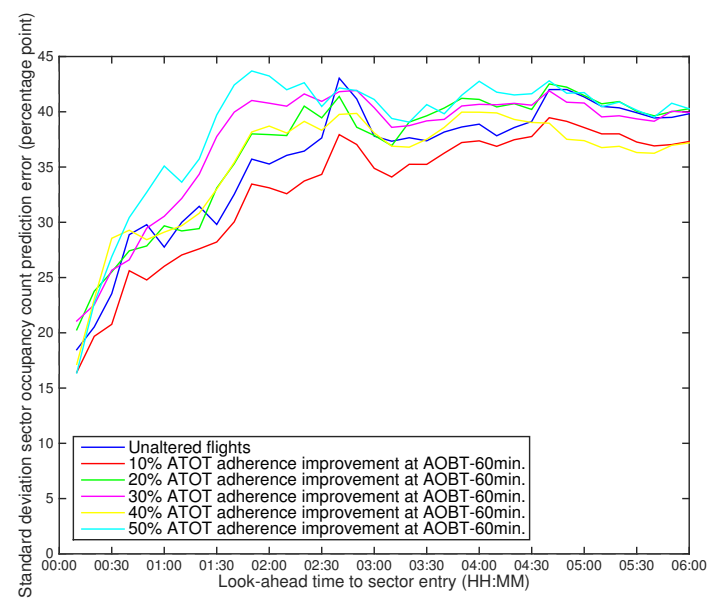

(d) Standard deviation, sector Nicky High

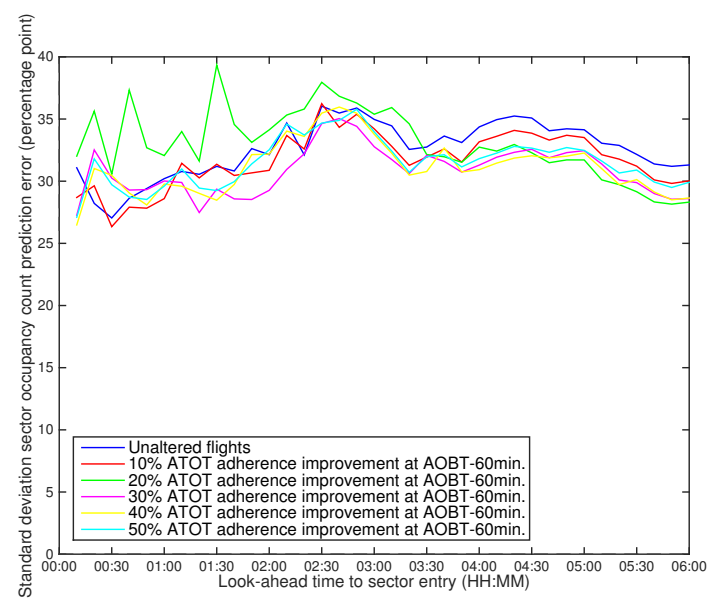

(f) Standard deviation, sector Lux Low

Figure 8.5: Sector occupancy count prediction error sensitivity for 3 sectors. Departure time error reduction done at 1 hour before off block. 


\subsection{Arrival Time Predictability}

To conclude the analysis, this section discusses sensitivity of standard deviation arrival time prediction error. Calculation of standard deviation arrival time prediction error for flights with $\sim 1$ hour flight time is explained in section 7.5. Table 8.4 shows that standard deviation of arrival time prediction error can be reduced up to 5 minutes if the pilot sticks $50 \%$ better to the planned take-off time at 1 hour before off block time. Overall the obtained improvements are relatively small. A graphical representation of this table is given in figure 8.6.

Table 8.4: Standard deviation arrival time prediction error sensitivity. Time format is $\mathrm{H}: \mathrm{MM}: \mathrm{SS}$.

\begin{tabular}{|c|c|c|c|c|c|c|c|}
\hline \multirow[b]{2}{*}{ At } & \multirow{2}{*}{$\begin{array}{l}\text { Time before } \\
\text { take-off }\end{array}$} & \multicolumn{6}{|c|}{ Departure time prediction error reduction } \\
\hline & & $0 \%$ & $10 \%$ & $20 \%$ & $30 \%$ & $40 \%$ & $50 \%$ \\
\hline $\begin{array}{l}\text { Start of slot } \\
\text { allocation }\end{array}$ & 6:00:00 & $0: 25: 08$ & $0: 23: 47$ & $0: 23: 00$ & $0: 22: 08$ & $0: 21: 14$ & $0: 20: 41$ \\
\hline $\begin{array}{l}\text { End of slot } \\
\text { allocation }\end{array}$ & 2:20:00 & 0:20:51 & 0:20:03 & $0: 19: 26$ & $0: 18: 55$ & 0:18:08 & $0: 17: 42$ \\
\hline $\begin{array}{l}\text { Start of push } \\
\text { back (off block) }\end{array}$ & 0:20:00 & $0: 12: 53$ & $0: 11: 42$ & $0: 11: 16$ & 0:10:41 & 0:10:08 & 0:09:50 \\
\hline Take-off & 0:00:00 & 0:08:57 & 0:07:22 & $0: 07: 12$ & 0:07:01 & 0:06:52 & 0:06:40 \\
\hline
\end{tabular}

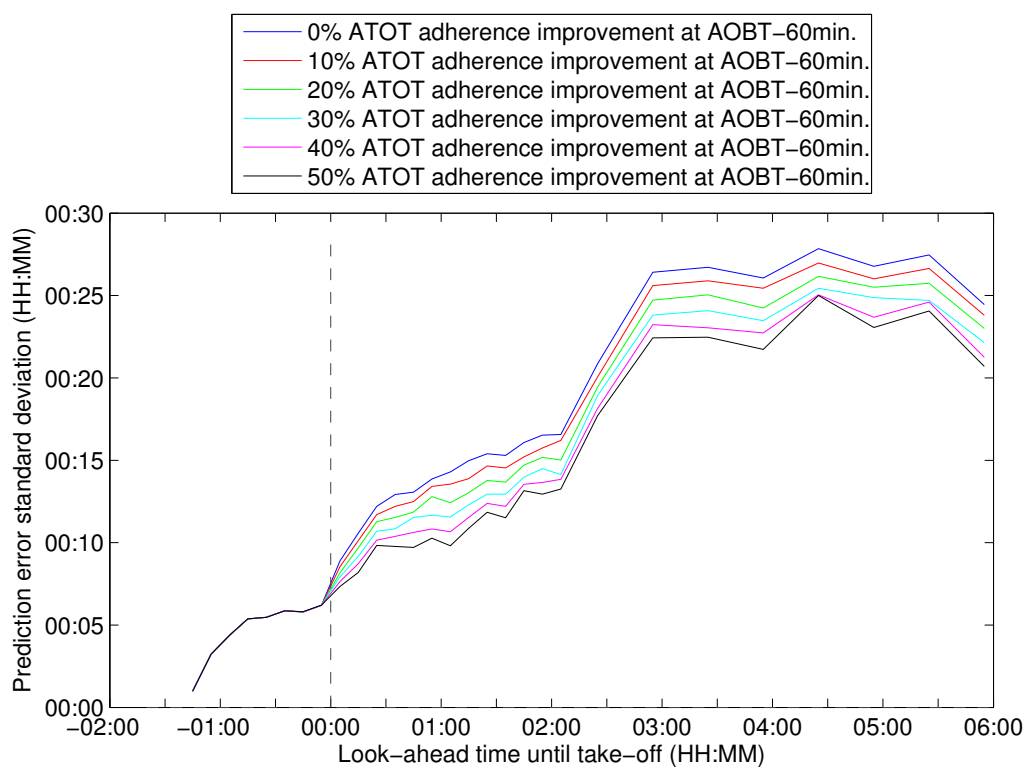

Figure 8.6: Standard deviation arrival time prediction error sensitivity for flight with 1 hour flight time. Departure time error reduction done at 1 hour before off block. 


\subsection{Conclusions}

This section highlights the main points of this chapter on the impact of improved departure time predictions relative to sector demand \& arrival time predictability:

- Entry \& arrival time prediction error for unimproved and improved flights after take-off are similar. This is because predicted and actual entry \& arrival times are changed with the same value after take-off.

- It is functional to reduce departure prediction error. If the pilots adheres $50 \%$ better to planned departure times at off block time, an improvement of $\sim 50 \%$ for mean non-absolute entry time prediction error, and $\sim 30 \%$ for mean absolute entry time prediction error, can be obtained.

- Sensitivity for mean prediction error of entry time \& occupancy count are similar. This indicates that taxi-time predictions are a major source of uncertainty.

- Improvement of departure time predictions in some cases results in larger mean sector occupancy count prediction error. These findings are rather disappointing because the used method does not yield realistic sector occupancy count because the inflow/outflow ratio is unrealistically altered. A possible explanation is that ATC procedures and improved predictability are interdependent, and improvement of departure time predictions alone does not yield realistic prediction count error. Sensitivity analysis results therefore need to be interpreted with caution.

- Standard deviation of arrival time prediction error can be reduced up to $20 \%$ if the pilot sticks $50 \%$ better to the planned take-off time at 1 hour before off block time. This is a relatively small reduction for a large effort. 


\section{Performance Impact \& Recommendations}

This chapter starts with summarizing the key findings of the analysis, and concludes with recommendations based on the analysis.

\subsection{Performance Impact}

Chapter 4 analyzes the number of flights as function of departure time prediction error.

- Figure 4.8 shows that mean \& standard deviation of departure time prediction error at initial flight plan are $6 \& 14$ minutes for non-regulated flights. Mean \& standard deviation for regulated flights are worse; 18 \& 31 minutes (figure 4.9). This can be explained by the discrepancy between the filed departure times by Aircraft Operators and ATFCM regulated departure times.

- It can be seen from figure 4.8 that mean \& standard deviation of the sector entry time prediction error at the initial flight plan are $4.5 \& 14.5$ minutes, which is better than the mean \& standard deviation of departure time prediction error at the initial flight plan. Sector entry time predictions are better than departure time predictions for both non-regulated as regulated flights. This can be explained by pilots speeding up / slowing down to recover the delay at departure and adhere to the flight plan.

- From figure 4.6 we can see that non-regulated flights on average depart later than planned (mean departure time prediction error is positive at several time instances before take-off). Figure 4.7 shows a peak of regulated departure time prediction error for flights at the beginning of the departure slot, this means that regulated flights tend to depart as soon as allowed to recover delay. 
- Figure 4.10 shows the average standard deviation of the departure time prediction error per airport. This prediction error is relative to the prediction at 30 minutes before take-off. Take note that the error distribution is dependent on the prediction look-ahead time. Most airports have a standard deviation of departure time prediction error of 9 minutes (non-regulated flights). This means that $\sim 68 \%$ of all flights depart not more than 9 minutes earlier, or later than the mean departure time prediction error which is $\sim 2.5$ minutes (figure 4.6). This is good because \pm 9 minutes falls within the departure time tolerance window of 15 minutes.

Following departure time predictability, chapter 5 analyzes the metrics; sector entry time, sector occupancy count, and sector entry count as function of look-ahead time to sector entry.

- Figure 5.6 illustrates that prediction error for flights with longer flight times to the sector is less dispersed than flights with short flight time to the sector. Longer flight time to the sector allows the pilot to speed up / slow down to adhere to the planned entry time.

- On a busy day, flights are more probable to be delayed for a look-ahead longer than 2 hours (figure 5.9a)

- Absolute sector entry time prediction error (variation of the error) increases linearly from 0 to 3 hours look-ahead, then it stabilizes around 7 minutes (figure 5.9)

- Figure 5.11 illustrates that at 0 to 3 hour look-ahead, all the flights that intend to enter the sector have filed the flight plan.

- It can be seen from figure 5.20 and 5.35 that most analyzed sectors show a constant mean entry \& occupancy count over-prediction of $\sim 15 \%$ for a look-ahead of 0 to 3 hours. However, sector Koksy High and Lux Low show a mean entry count underprediction. Count over-prediction means that there are more flights anticipated than really entered.

- Mean entry \& occupancy count error are not reduced to zero just before sector entry figure 5.20 and 5.35). This is due to flights being earlier or delayed, or due to route deviations, just before sector entry. Looking at the time period from 0 to 10 minutes before entry, it can be seen that some forecasted flights did not enter in the time of day window anymore (outflow), and some additional flights entered in the window that were initially not forecasted (inflow). Looking at 10 minutes before entry, about $30 \%$ to $40 \%$ are in/out flights (appendix D.1 ), which is a large amount.

- In general, for a look-ahead of 0 to 3 hours, there are more outflow than inflow flights resulting in the before mentioned over-prediction. The inflow/outflow flights ratio (stated in table 5.5) determines the mean sector occupancy count prediction error (illustrated in figure 5.20a).

Then turning to arrival time predictability in chapter 7 , the following key findings were made: 
- Flight phases along the flight time horizon that show relatively poor prediction accuracy are; the slot allocation process, the taxi phase, and the descent phase. These phases are considered to have poor prediction performance because the standard deviation arrival time prediction error in figure 7.4 illustrates steep curve sections for these phases.

- Prediction uncertainty in the slot allocation process can be explained by the discrepancy between initially flight filed plans by Airspace Users, and the actually allocated departure slots by the ETFMS. The contribution of this uncertainty is $18 \%$ of the total arrival time uncertainty at 6 hours before take-off (figure 7.4).

- Prediction uncertainty in the taxi phase can be explained by discrepancy between the average predicted taxi-time for an airport which holds for all flights, and the real taxi time that differs from flight to flight. The contribution of this uncertainty is $11 \%$ of the total arrival time uncertainty at 6 hours before take-off (figure 7.4).

- Prediction uncertainty in the descent phase can be explained by discrepancy between the predicted and actual standard terminal arrival route. The contribution of this uncertainty is $21 \%$ of the total arrival time uncertainty at 6 hours before take-off (figure 7.4).

Finally, the performance impact of improved departure time predictions in chapter 8 is discussed:

- If the pilots adheres $50 \%$ better to planned departure times at off block time, an improvement of $\sim 50 \%$ for mean non-absolute entry time prediction error (figure 8.2 ), and $\sim 30 \%$ for mean absolute entry time prediction error (figure 8.3), can be obtained at look-ahead longer than 4 hours. This indicates that taxi-time predictability has a large influence on sector entry time predictability.

- Sensitivity for mean prediction error of entry time (figure 8.3) \& occupancy count (figure 8.4) are similar. This indicates that taxi-time predictions are a major source of uncertainty.

- One unanticipated finding is that improvement of departure time predictions in some cases results in larger mean sector occupancy count prediction error. For example sensitivity for mean sector occupancy count prediction error in figure 8.5a; the count error for a $40 \%$ departure time prediction improvement is closer to the 0 error line than the count error for a $50 \%$ departure time prediction improvement. These findings are rather disappointing because the used method does not yield realistic sector occupancy count. Sensitivity of occupancy count should therefore be interpreted with caution.

- It is evident from table 8.4 that standard deviation of arrival time prediction error can be reduced up to $\sim 20 \%$ at 6 hours before take-off, if the pilot sticks $50 \%$ better to the planned take-off time at 1 hour before off block time. 


\subsection{Recommendations}

Based on the analysis, the following recommendations are made:

- Figures on prediction error of entry time (figure 5.9) \& occupancy count (figure 5.20), show benchmark curves highlighting sectors with relatively poor prediction performance. Future investigations should consider the low performing sectors, and analyze causes to improve predictability.

- On a busy day, the Flow Manager should anticipate that flights have a higher probability to be delayed (figure 5.9), at a look-ahead of 2 hours and longer. Furthermore he/she should anticipate, taking safety into account, for a constant sector occupancy count over-prediction of $\sim 15 \%$, for 0 to $3 \mathrm{~h}$ look-ahead (figure 5.20 ).

- Table 5.5 shows for all sectors that 'inflow flights due to time deviation' < 'outflow flights due to time deviation'. The reason for this is unknown, and further research is suggested. A possible explanation could be the uncertainty in sector crossing duration predictions.

- Table 5.5 shows that sectors with large over-prediction have a large number of outflow flights due to route deviation. In order to reduce over-prediction for these sectors, outflow flights due to route deviation should be reduced. For Koksy Low on the 5th of March 2014, a 5\% decrease in outflow flights due to route deviations, results in $10 \%$ less over-prediction. As vertical route uncertainty causes $70 \%$ of the uncertainty problems on operations [5], and ensuring that safety is kept at the level of today, operationally unnecessary level changes should be reduced.

- It is evident from table 8.4 that standard deviation of arrival time prediction error can be reduced up to $\sim 20 \%$ at 6 hours before take-off, if the pilot sticks $50 \%$ better to the planned take-off time at 1 hour before off block time. This improvement is fairly small for a relatively large effort. To improve (arrival time) predictability it is suggested that departure time predictions are improved as much as possible together with improvement of the flight phases that show relatively poor prediction performance (descent, taxi, and slot time allocation process) as quantified in section 8.5.

- Improvement of departure time predictions in some cases results in larger mean sector occupancy count prediction error, see figure 8.5a. These findings are rather disappointing because the used method does not yield realistic sector occupancy count because the inflow/outflow ratio is unrealistically altered. A possible explanation is that ATC procedures (level clearances, direct routing) and improved predictability are interdependent, and improvement of departure time predictions alone does not yield realistic prediction count error. Sensitivity analysis should include the effect of ATC procedures due to improved predictability, therefore it is suggested to used Fast Time Simulations. 


\section{Chapter 10}

\section{Conclusions}

In this chapter conclusions are made concerning the thesis objectives, based on research results. Subsequently limitations of the study are given, and recommendations are made for future work.

\subsection{Conclusions}

This thesis provides a quantification of the predictability of departure time, sector demand, and arrival time. Contributing factors for the sector demand are entry time, entry count, and occupancy count, the latter is examined in more detail. The actual sector occupancy count is validated with the software tool NEST. The impact of improved departure time prediction relative to sector demand \& arrival time predictability is analyzed. The results of this thesis provide a general overview of the current prediction accuracy, and give recommendations on improving predictability. This is in accordance with one objective among others, of the European ATM research development program SESAR, to increase trajectory prediction accuracy.

As expected, the accuracy of departure time predictions increase as we move closer to departure time. Regulated flights have larger departure time prediction error at initial flight plan than non-regulated flights. The sector entry time prediction error at the initial flight plan, is better than the departure time prediction error at the initial flight plan, indicating that the pilot speeds up/slows down to adhere to the flight plan. This holds for both regulated and non-regulated flights.

For the predictability of sector entry time, this study shows that on a busy day, for a look-ahead of 2 hours and longer, flights have a higher probability to be delayed than to be earlier than planned. The absolute prediction error (indication of the error variation) increases linearly from 0 to 3 hours look-ahead, and after 3 hours the error stabilizes at 8 minutes, which is considered as good. On a busy day the sector entry time prediction error is larger than on a normal day. 
Most analyzed sectors show a mean entry \& occupancy count over-prediction from a look-ahead of 3 hours and earlier. However, sector Koksy High and Lux Low show a mean entry count under-prediction. The mean count error stabilizes between 0 to 3 hours look-ahead because almost all flight plans have been filed. Findings show that relative sector performance differ, based on the analyzed metric i.e. entry time, entry count, or occupancy count. Mean entry \& occupancy count error are not reduced to zero just before sector entry. This is due to flights being earlier or delayed, or due to route deviations, just before sector entry. These flights that deviate from the planned trajectory are called inflow and outflow flights. In general, prediction volatility is high as $30 \%$ to $40 \%$ are inflow and outflow flights at 10 minutes before sector entry. The inflow/outflow flights ratio determines the mean sector occupancy count prediction error. For example an overprediction relates to a situation with less inflow than outflow flights.

Flight phases with relatively large amounts of prediction uncertainty are; descend (e.g. Standard Terminal Arrival Routes uncertainty), taxi (e.g. runway congestion), and the slot allocation process (e.g. discrepancy between allocated departure slot and initial flight plan).

Sensitivity of the improved departure times show fairly small reductions of the standard deviation of the arrival time prediction error; 20\% standard deviation arrival time reduction for a $50 \%$ departure time prediction improvement.

Sensitivity for mean sector entry time prediction error at off block time, and 1 hour before off block, are similar, indicating that taxi-time predictions are a major source of uncertainty. If pilots adhere $50 \%$ better to planned departure times at off block time, a reduction of $\sim 50 \%$ of the mean entry time prediction error, and $\sim 30 \%$ of the mean absolute entry time prediction error, can be obtained which indicate that better adherence leads to improvement of sector entry time predictions.

One unanticipated finding is that improved departure time predictability in some cases results in a larger mean occupancy count prediction error. A possible explanation is that ATC procedures (direct routing, level capping, etc.) and improved predictability are interdependent. These findings are rather disappointing because the used method does not yield realistic sector occupancy count, and results of should be interpreted with caution.

\subsection{Limitations \& Future Work}

Sector demand predictability is analyzed for a busy day and normal day. Inferences about predictability for a day with small traffic levels cannot be made.

In order to obtain less over-prediction it is suggested to reduce 'outflow flights due to route deviations'. This can be done if ATC do not allow operationally unnecessary level or route changes, or if Airspace Users do not request operationally unnecessary level or route changes. These measures cause less flexibility for ATC and Airspace Users.

There is a discrepancy between the actual count obtained by this thesis algorithm and by software tool NEST. The first reason for this discrepancy is that the input flight data file for the algorithm does not include flights that have sector crossing durations of $<1$ 
minute, while NEST does. Second, a limitation of the thesis algorithm is that it assumes that the flight has entered the sector if a flight prediction update states that; (1) the flight has entered the sector, but (2) it has not yet departed.

As mentioned before, sensitivity of improved departure time predictions relative to sector demand \& arrival time predictability does not include the effect of ATC procedures due to improved predictability. Therefore caution must be applied in interpreting the results, as the findings might not be an accurate depiction of real life events.

Three directions for future research are recommended:

- An considerable amount of research is already available on ATC workload models, further research should be focused on practical applications of ATC workload predictability. For example taking the 4D trajectory predictions from historical data and modeling workload predictions, and then validating these models with real measured workload from real time simulations.

- Investigation into practical applications of probabilistic forecasting should be done, as the current flow management system could more involve the stochastic nature of predictions. This could be an important part of the next step of predictability improvement in the SESAR 2020 context.

- It is recommended to quantify the relationship between count predictability and ATC procedures. Recorded ATC procedures are not readily available, but the effect of ATC procedures can be seen trough horizontal \& vertical route deviations. 


\section{References}

[1] Eurocontrol, 2013. Enhanced Tactical Flow Management System [online] Available at: http://tinyurl.com/p5r8hfp [Accessed 16-11-2014].

[2] Frederik Michel Dekking. A Modern Introduction to Probability and Statistics: Understanding why and how. Springer, 2005.

[3] Eurocontrol. Network Strategic Tool, software, version 1.1, Brussels.

[4] Eurocontrol. A complexity study of the Maastricht Upper Airspace Centre. Technical Report 403, 2006.

[5] Eurocontrol. Benefits of improved predictability final report (d4) 1.0. Technical report, December 2012.

[6] Eurocontrol. Performance benefit mechanism and strategy for quantitative assessment for SESAR step 2. Technical report, December 2012.

[7] Eurocontrol. Departure time variability and TTO variability (study on regulated and non-regulated flights). Technical report, December 2013.

[8] Eurocontrol. Relating increases in sector capacity to reductions in entry time variance. Technical report, December 2013.

[9] Esther Calvo Fernández and José Manuel Cordero. Effect of flight plans predictability and accuracy on traffic demand forecast. In Proceedings of the 3rd International Conference on Application and Theory of Automation in Command and Control Systems, pages 46-55. ACM, 2013.

[10] Jimmy Krozel, Dan Rosman, and Shon R Grabbe. Analysis of en route sector demand error sources. In AIAA Guidance, Navigation and Control Conference, 2002.

[11] Kenneth S Lindsay, Daniel P Greenbaum, and Craig R Wanke. Predeparture uncertainty and prediction performance in collaborative routing coordination tools. Journal of guidance, control, and dynamics, 28(6):1178-1186, 2005. 
[12] Craig Wanke, Michael B Callaham, Daniel P Greenbaum, and Anthony J Masalonis. Measuring uncertainty in airspace demand predictions for traffic flow management applications. In AIAA Guidance, Navigation and Control Conference. Austin, TX, USA, 2003. 


\section{Appendix A}

\section{Flight Prediction Update Message}

The ETFMS outputs approximately 60 prediction updates per flight. These updates are contained in the ETFMS Flight Data file. Figure A.1 shows a single prediction update for the British Airways example flight as discussed in section 1.4. 

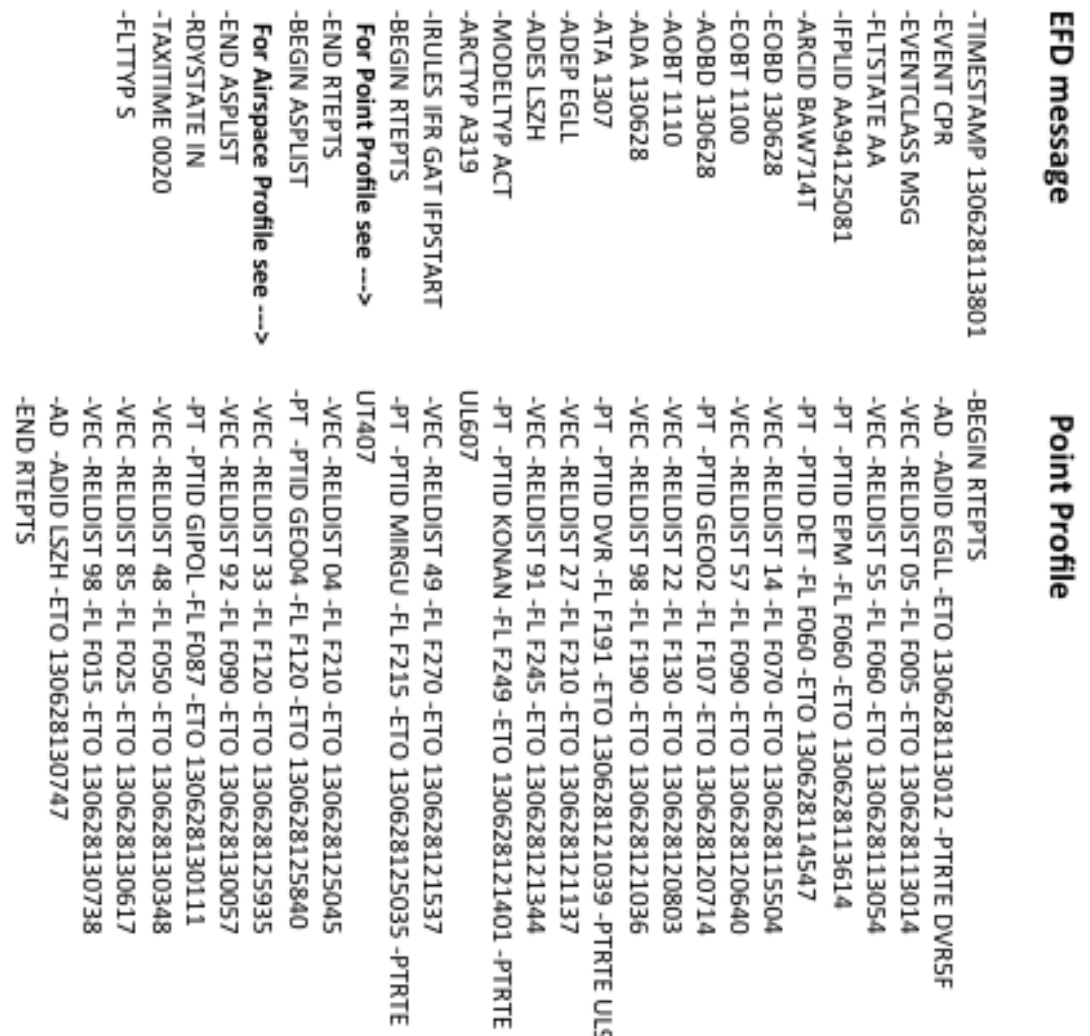

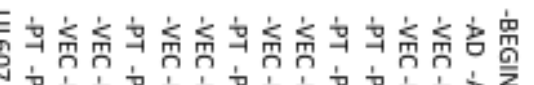

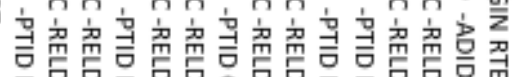

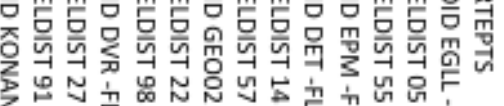

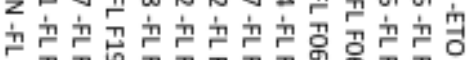

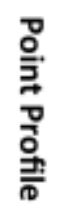

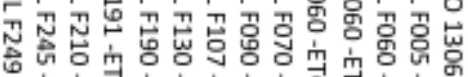

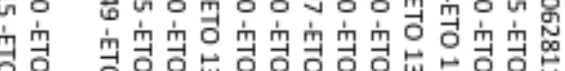

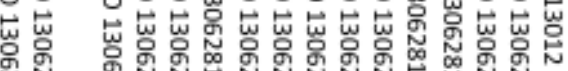
每 怘 峦

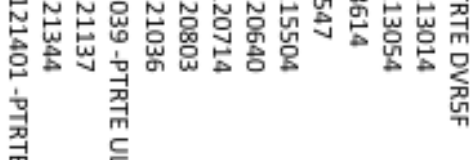

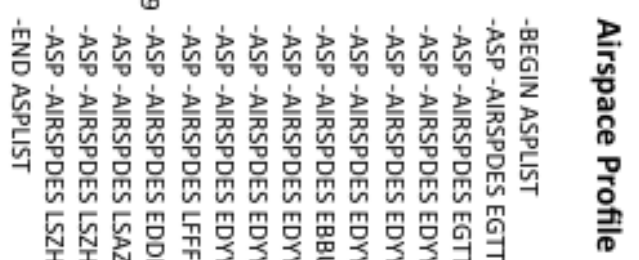

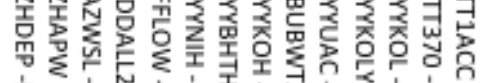

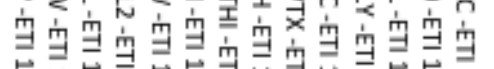

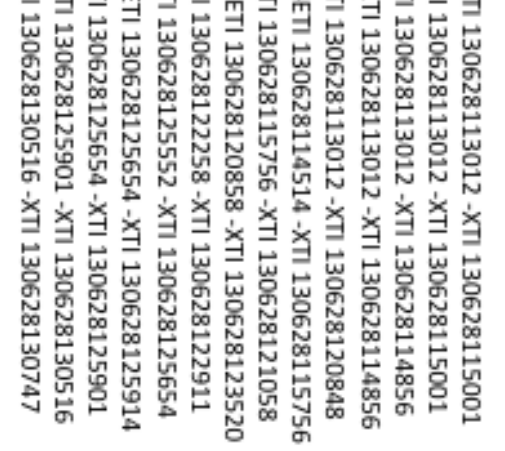

Figure A.1: Prediction update message for individual flight. 


\section{Appendix B}

\section{Departure Time Predictability for Normal \& Busy Days}

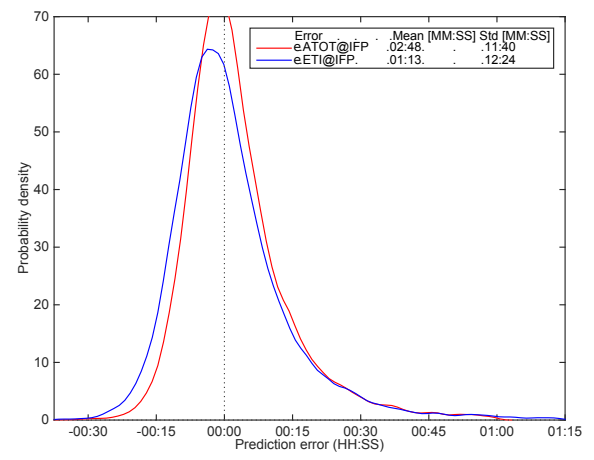

(a) Normal days

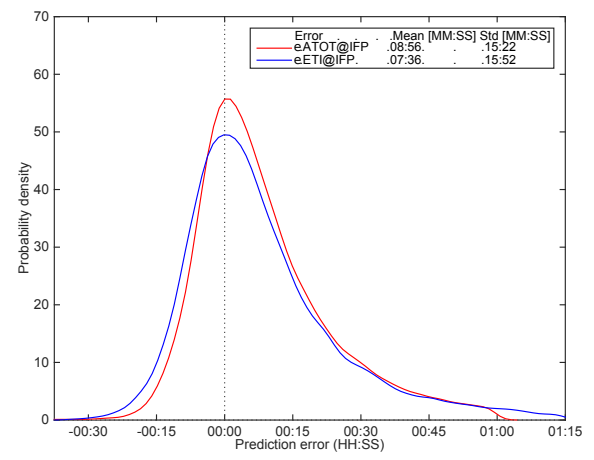

(b) Busy days

Figure B.1: Take-off time, and sector entry time prediction error at initial flight plan, nonregulated flights. 


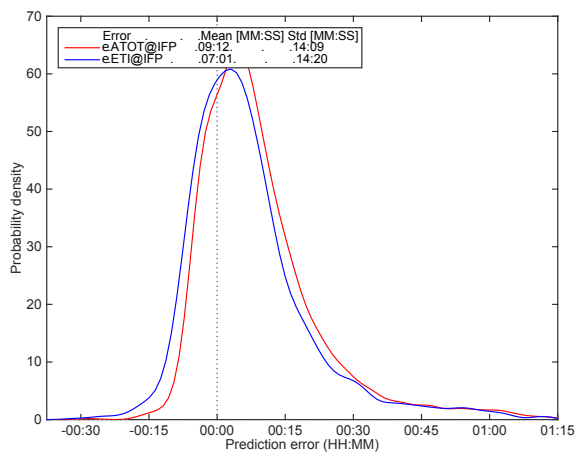

(a) Normal days

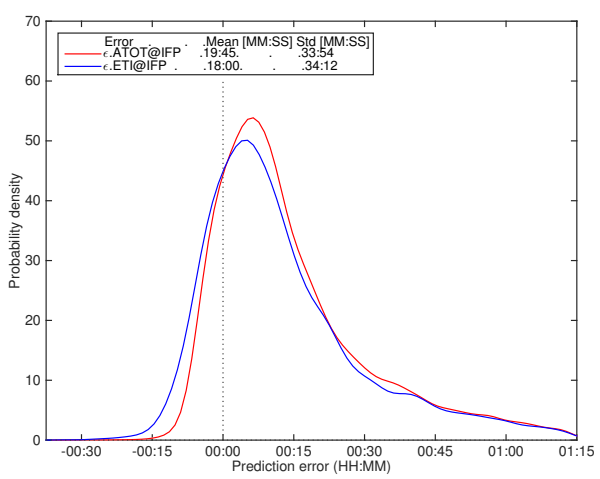

(b) Busy days

Figure B.2: Take-off time, and sector entry time prediction error at initial flight plan, regulated flights.

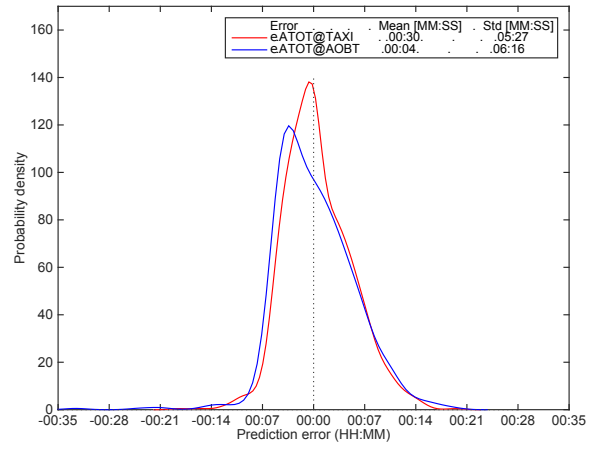

(a) Normal days

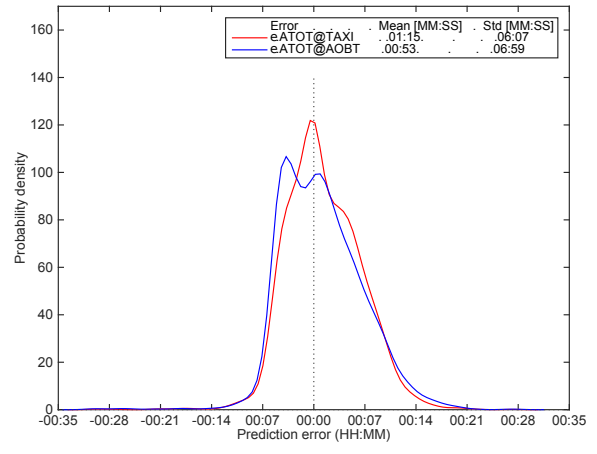

(b) Busy days

Figure B.3: Take-off time prediction error during taxi, and at off-block time, for regulated flights

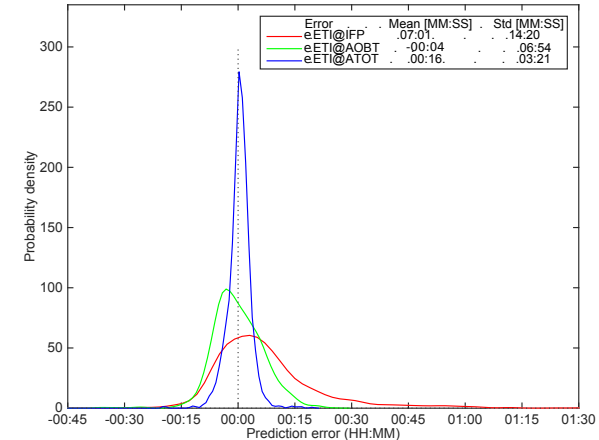

(a) Normal days

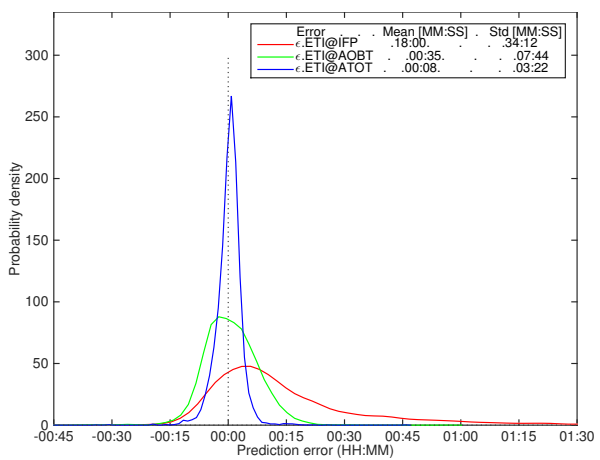

(b) Busy days

Figure B.4: Sector entry time prediction error at initial flight plan, at off-block time, and at take-off, for regulated flights 


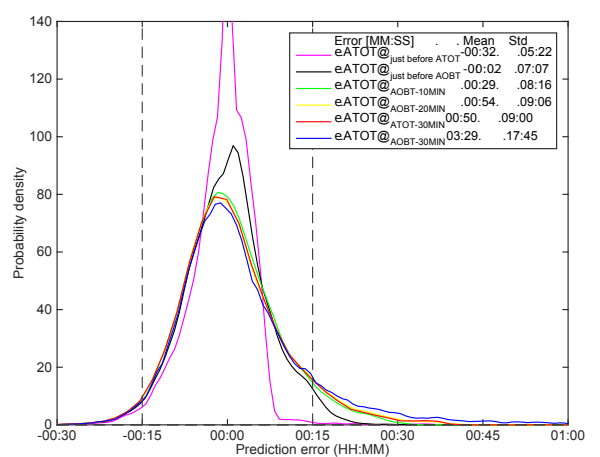

(a) Normal days

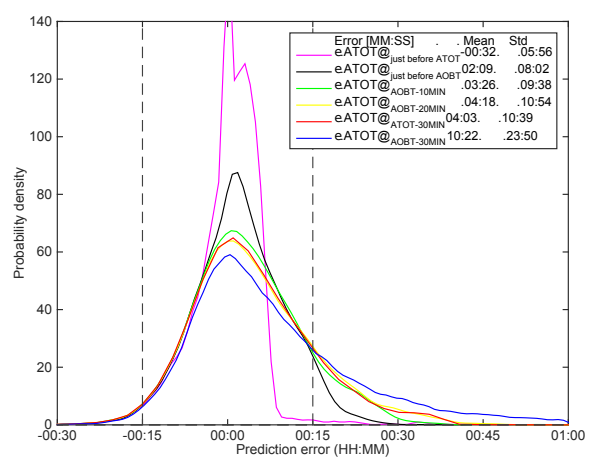

(b) Busy days

Figure B.5: Take-off time prediction error at different time instances for non-regulated flights.

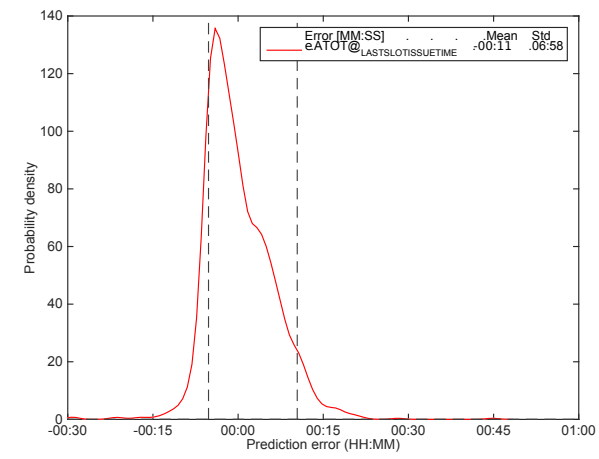

(a) Normal days

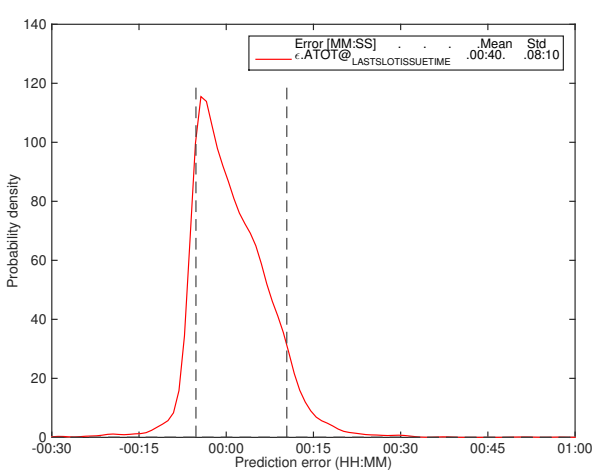

(b) Busy days

Figure B.6: Take-off time prediction error at last slot issue time for regulated flights

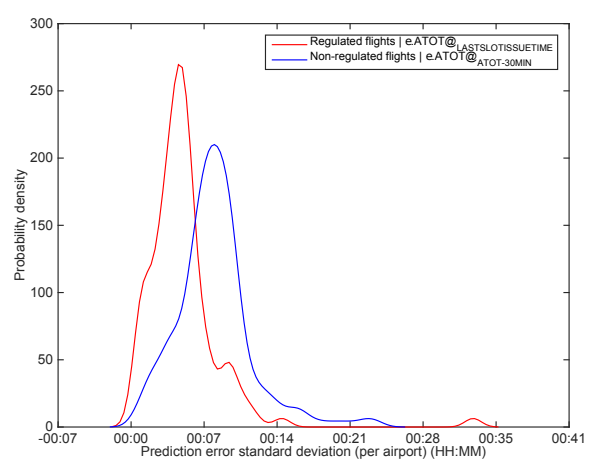

(a) Normal days

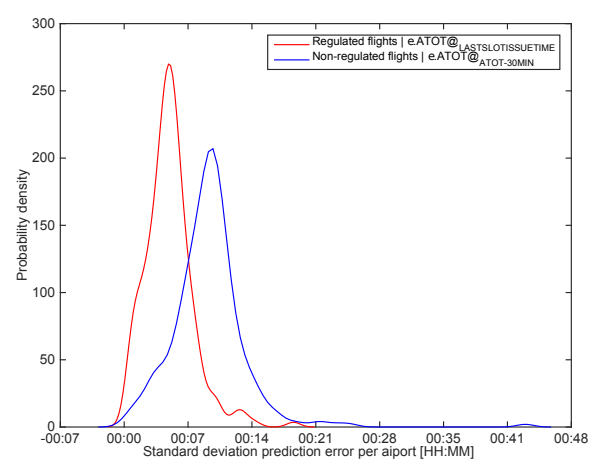

(b) Busy days

Figure B.7: Standard deviation of the departure time prediction error per airport. Error for regulated flights at last slot issue time, error for non-regulated flights at 30 minutes before take-off. 
Appendix C

\section{Sector Occupancy Count Flight Status at Prediction}




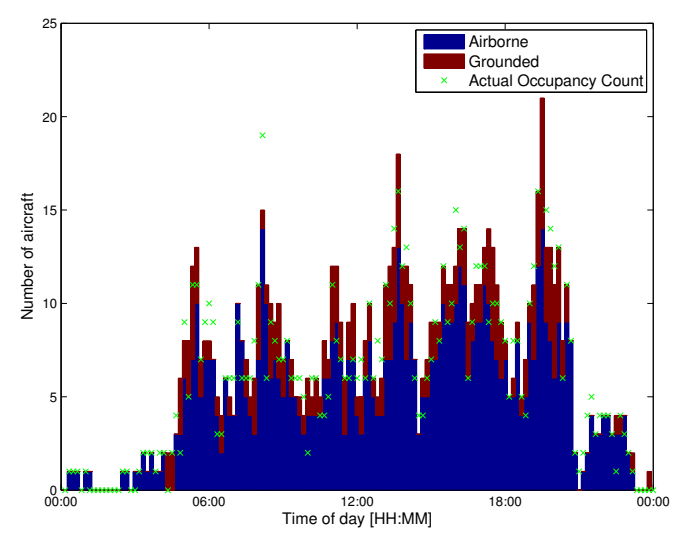

(a) Sector Jever Low.

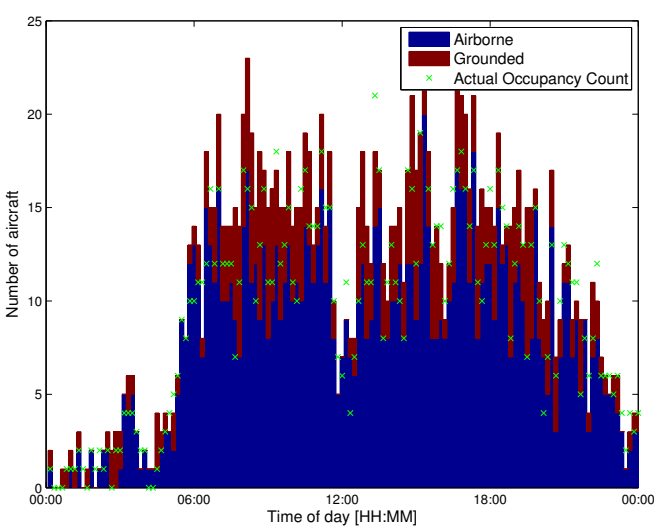

(c) Sector Koksy Low.

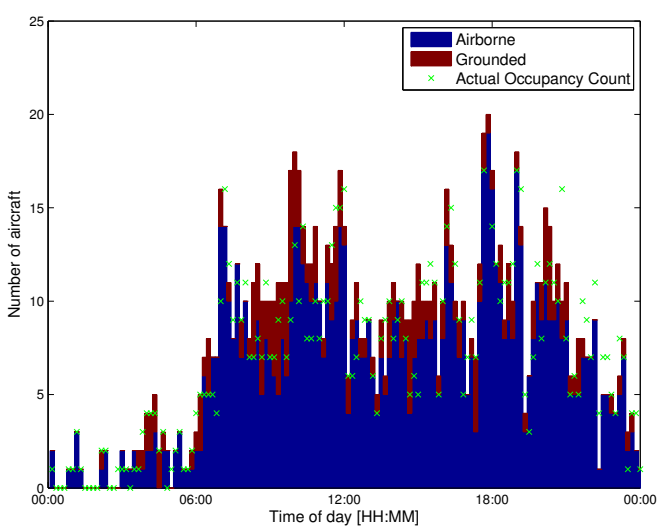

(e) Sector Koksy Low, 05/03/2014.

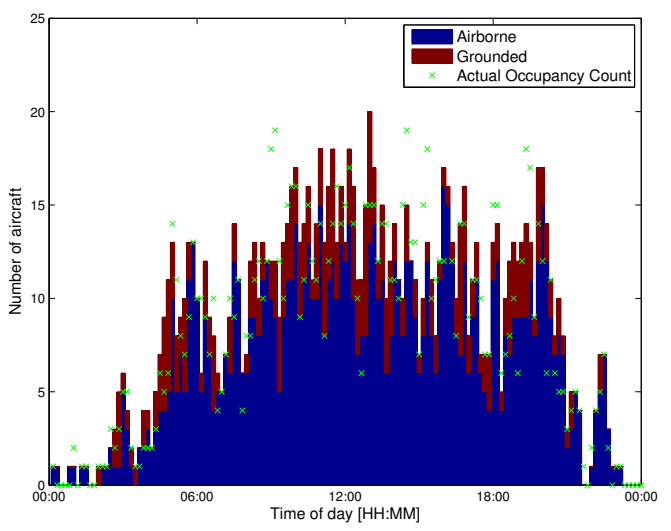

(g) Sector Lux Low.

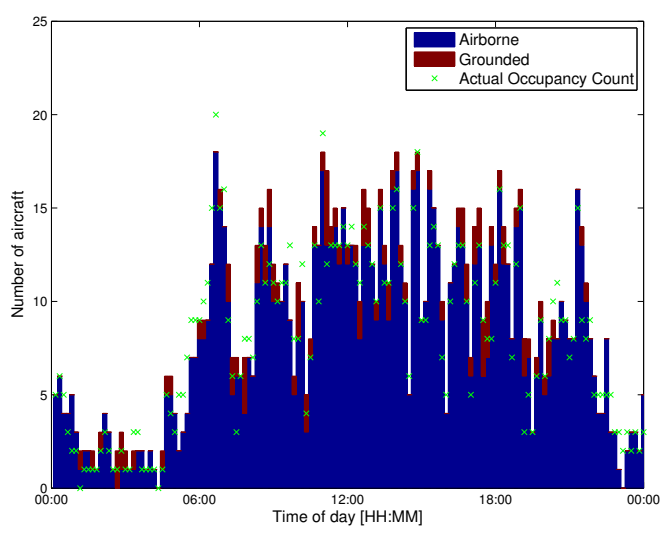

(b) Sector Koksy High.

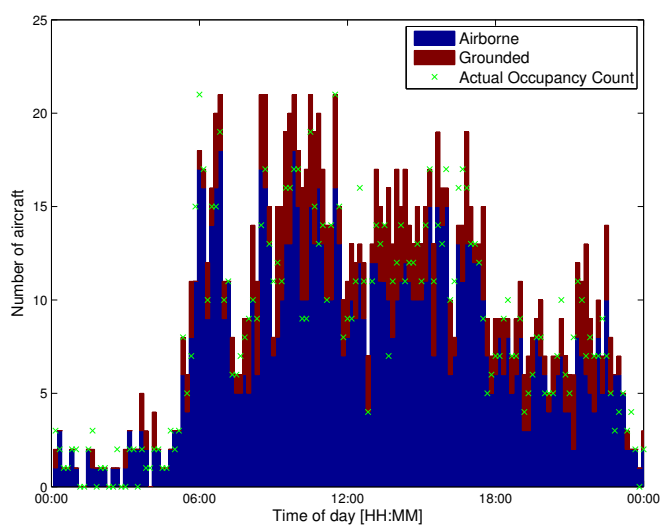

(d) Sector Koksy Low, 29/06/2013.

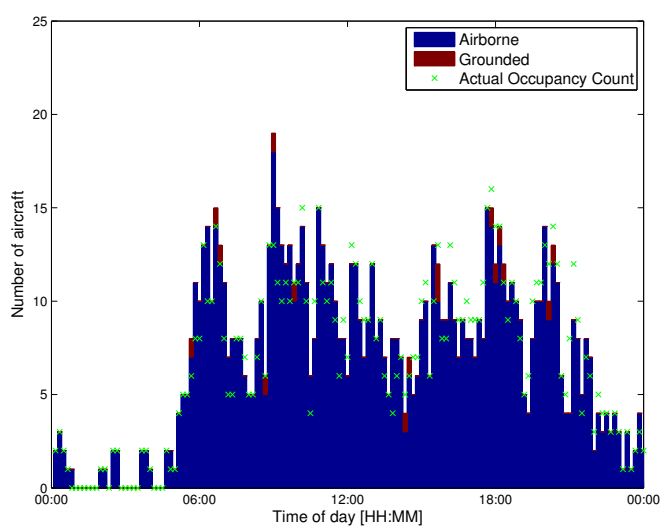

(f) Sector Bordeaux X4.

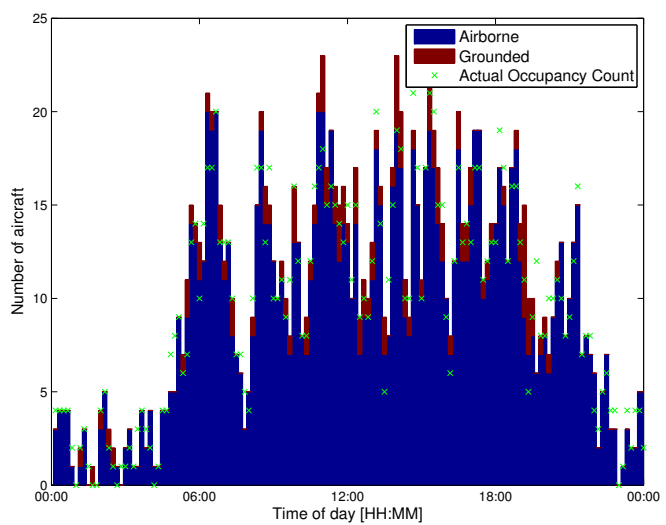

(h) Sector Nicky High.

Figure C.1: Sector occupancy count flight status at prediction for multiple sectors. Lookahead is 10 minutes. 


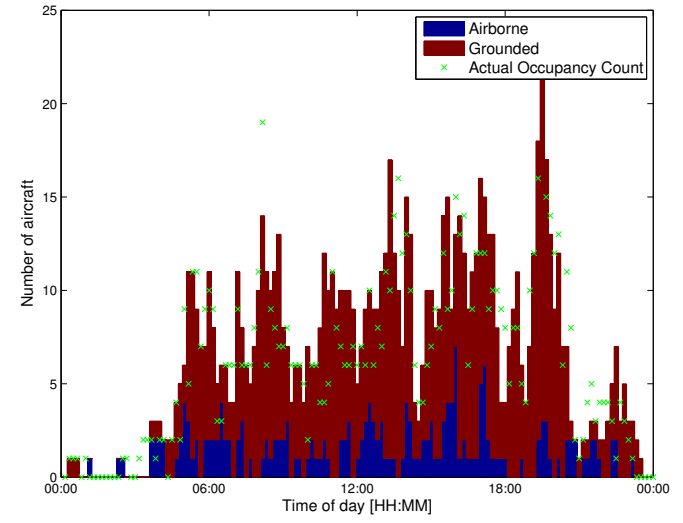

(a) Sector Jever Low.

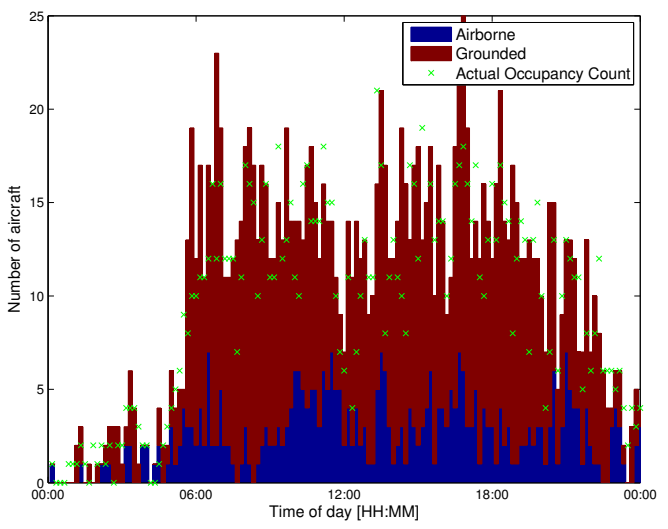

(c) Sector Koksy Low.

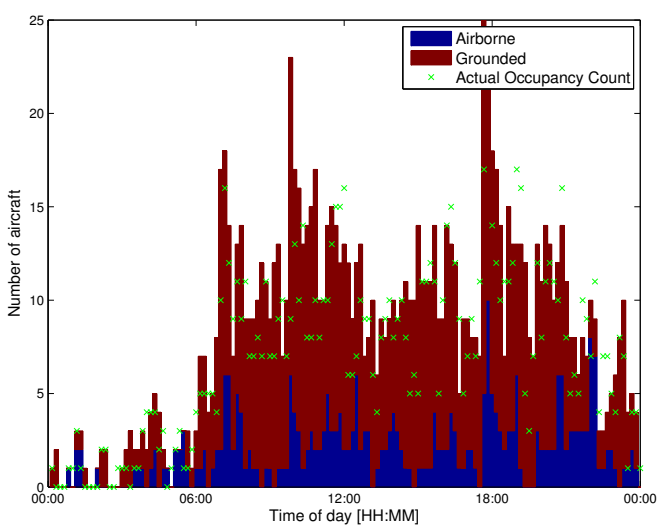

(e) Sector Koksy Low, 05/03/2014.

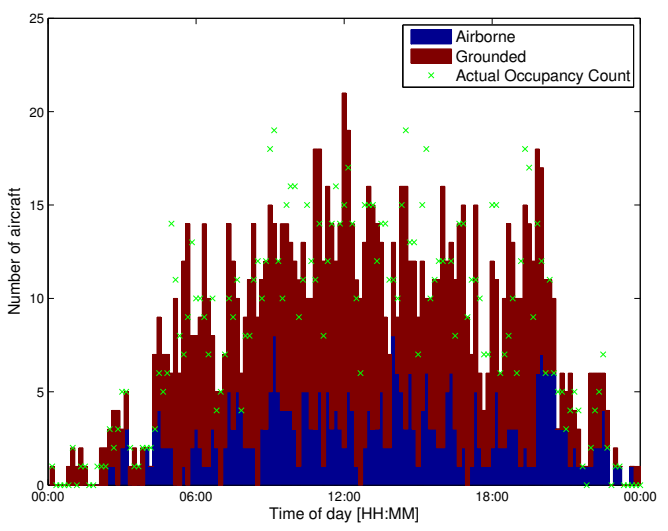

(g) Sector Lux Low.

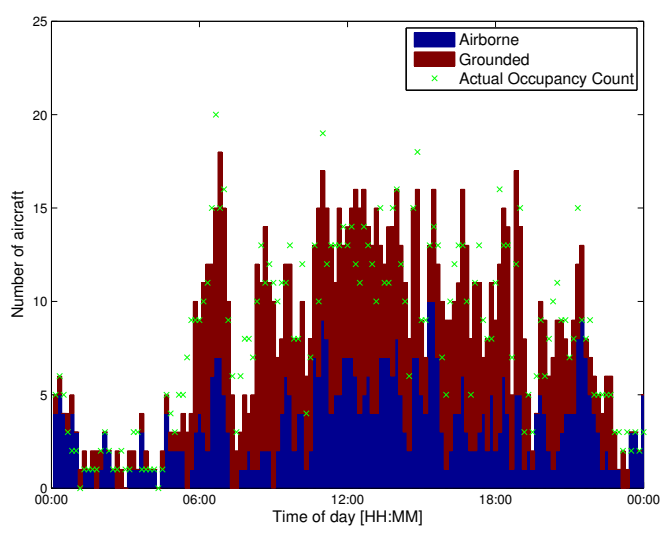

(b) Sector Koksy High.

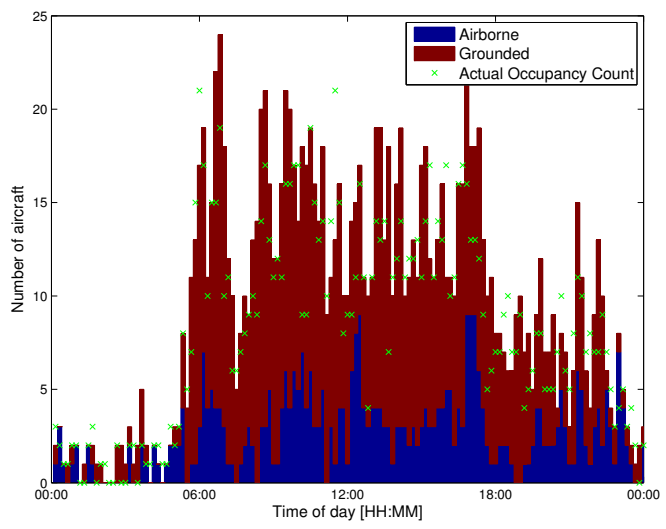

(d) Sector Koksy Low, 29/06/2013.

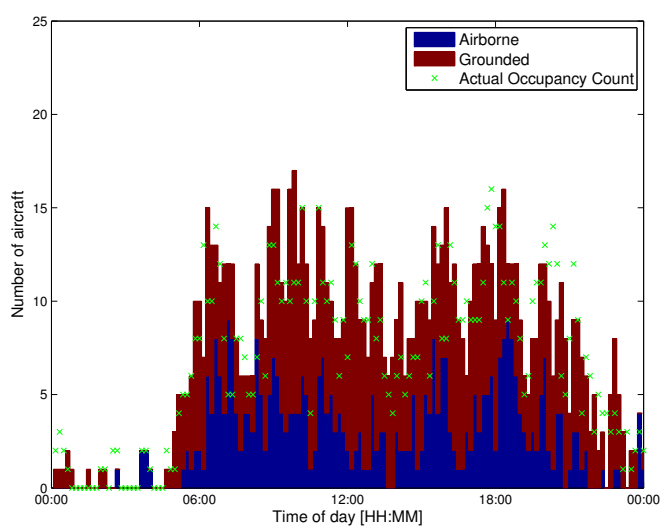

(f) Sector Bordeaux X4.

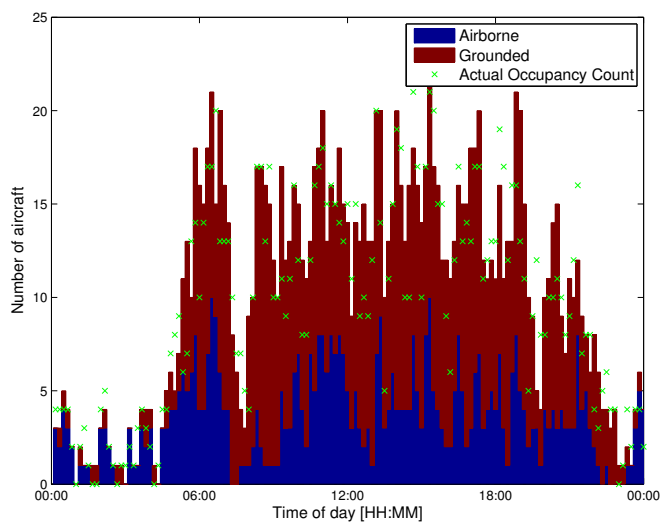

(h) Sector Nicky High.

Figure C.2: Sector occupancy count flight status at prediction for multiple sectors. Lookahead is 60 minutes. 


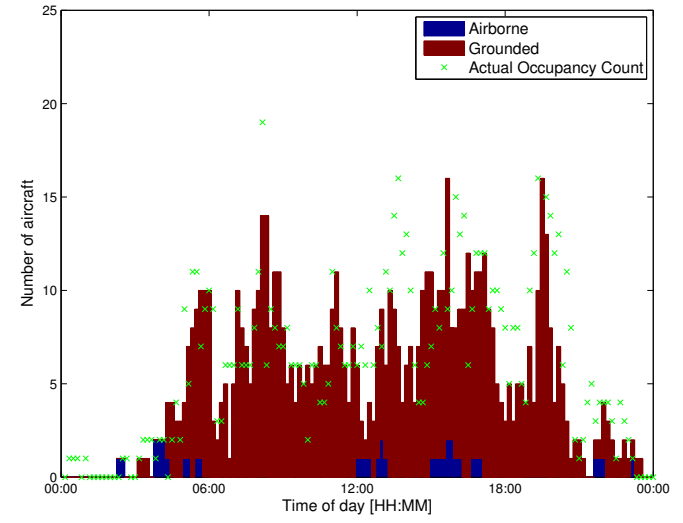

(a) Sector Jever Low.

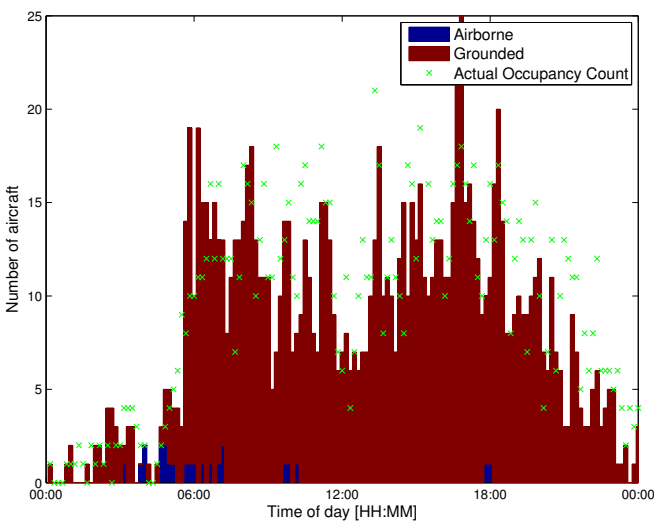

(c) Sector Koksy Low.

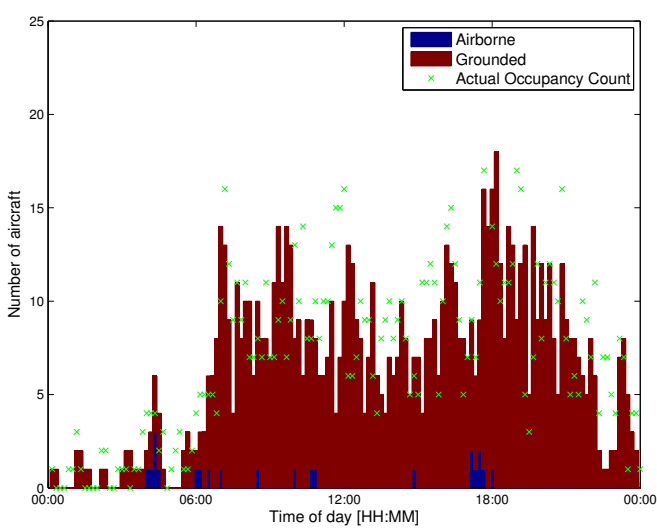

(e) Sector Koksy Low, 05/03/2014.

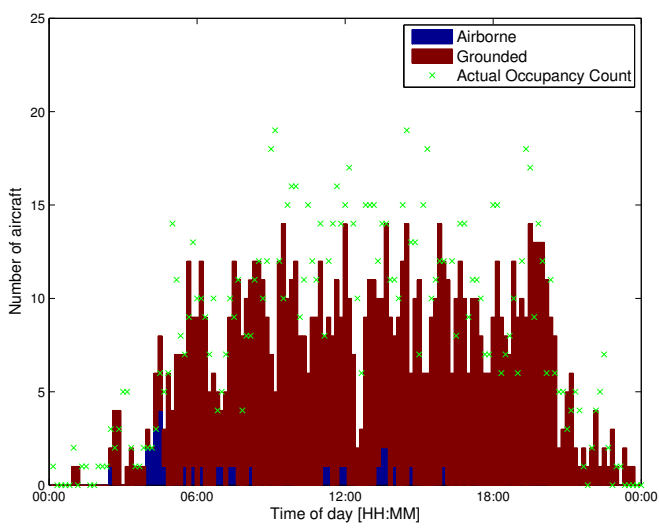

(g) Sector Lux Low.

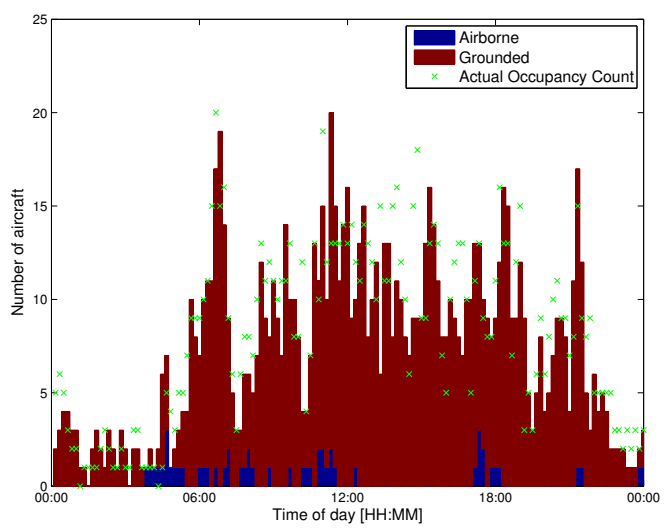

(b) Sector Koksy High.

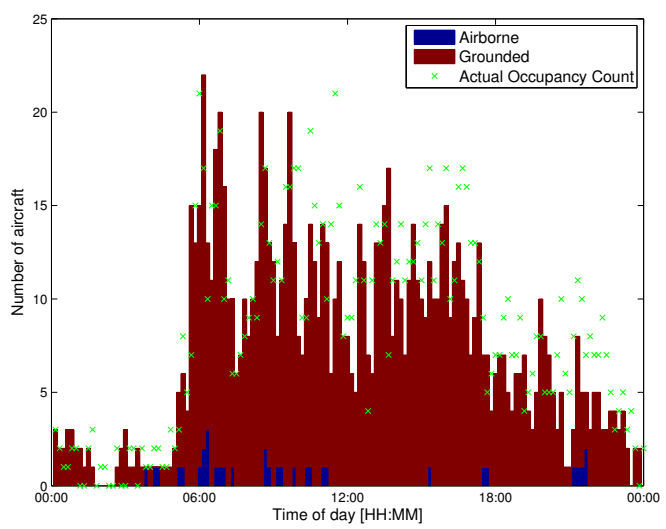

(d) Sector Koksy Low, 29/06/2013.

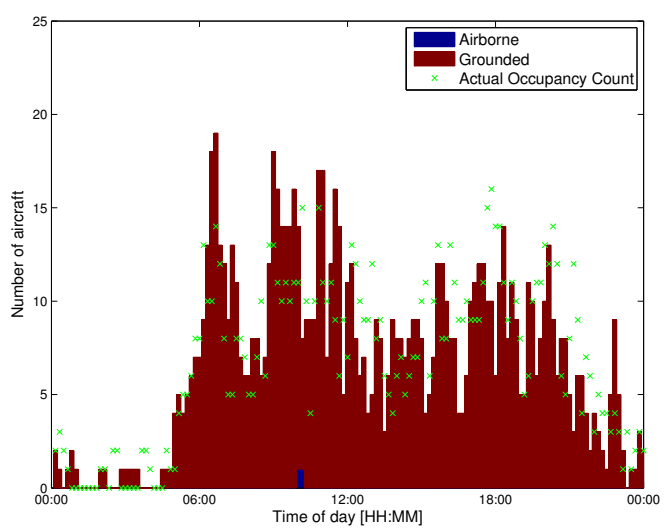

(f) Sector Bordeaux X4.

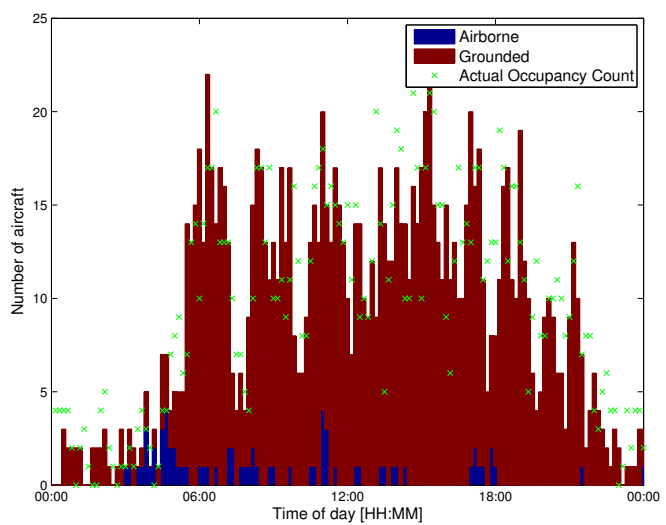

(h) Sector Nicky High.

Figure C.3: Sector occupancy count flight status at prediction for multiple sectors. Lookahead is 6 hours. 
Appendix D

Sector Occupancy Count Inflow \& Outflow Flights 


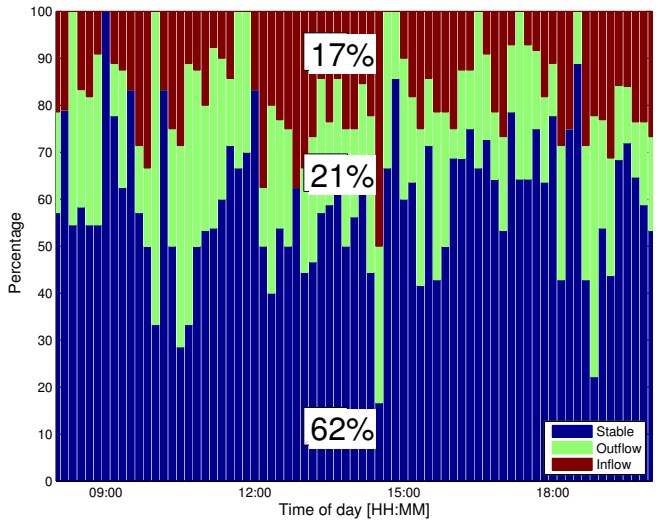

(a) Sector Jever Low.

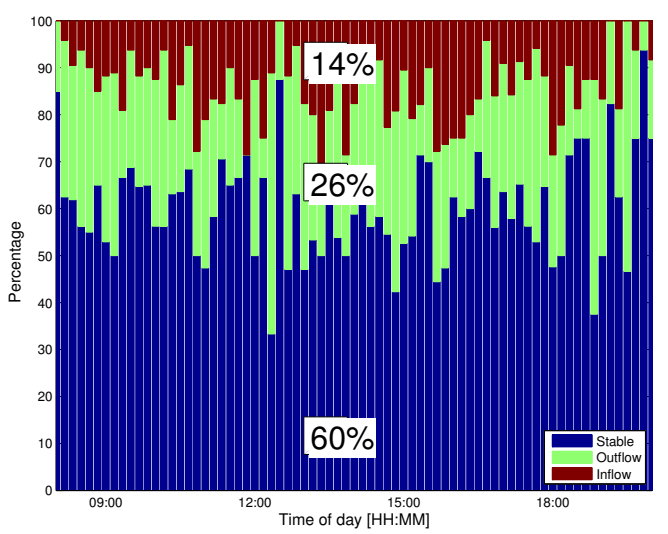

(c) Sector Koksy Low.

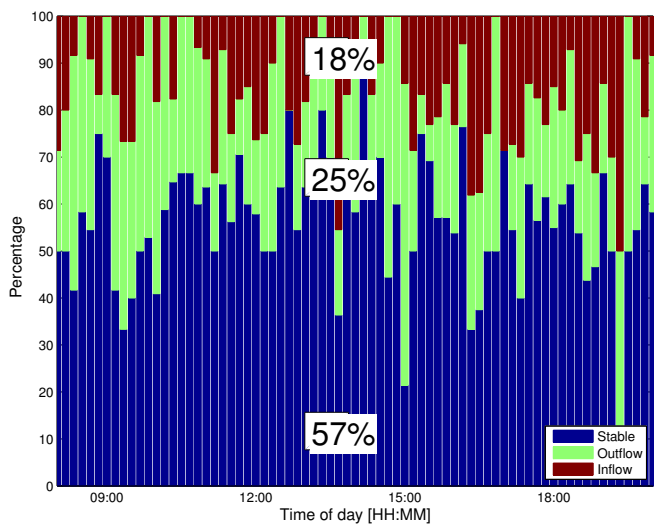

(e) Sector Koksy Low, 05/03/2014.

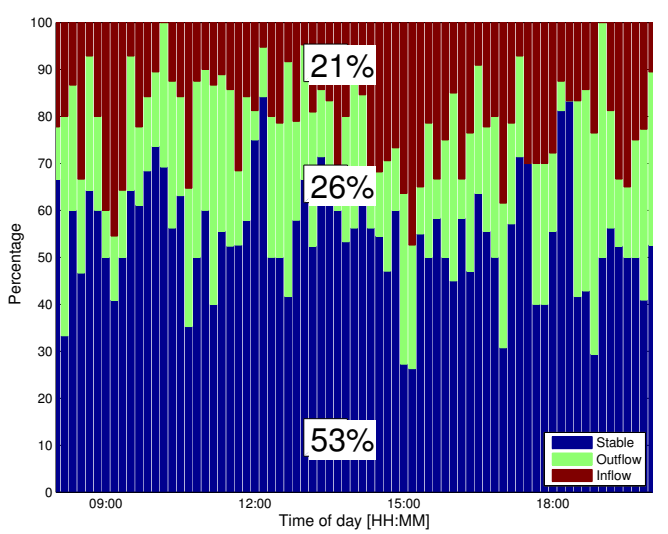

(g) Sector Lux Low.

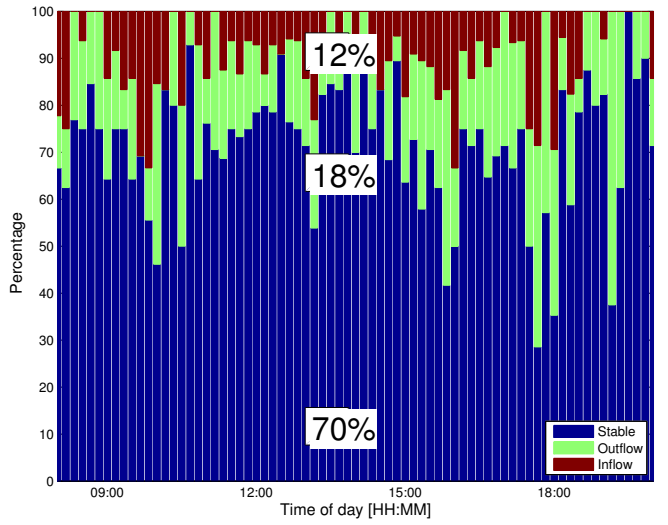

(b) Sector Koksy High.

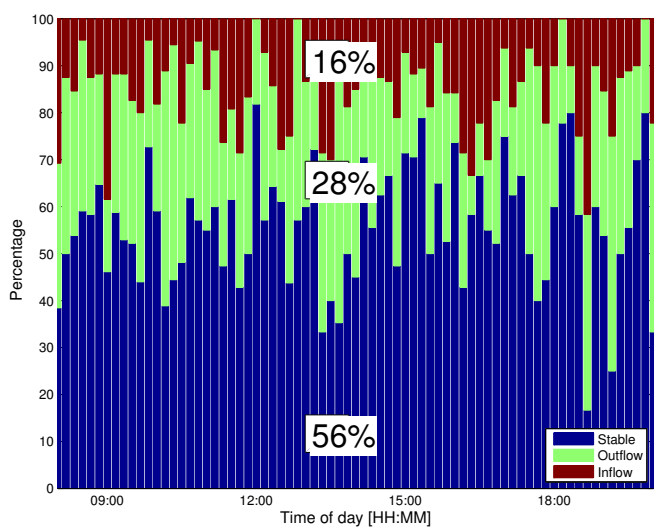

(d) Sector Koksy Low, 29/06/2013.

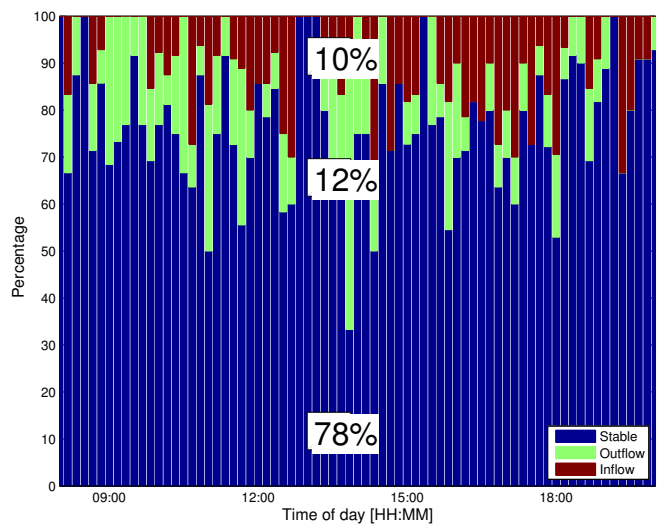

(f) Sector Bordeaux X4.

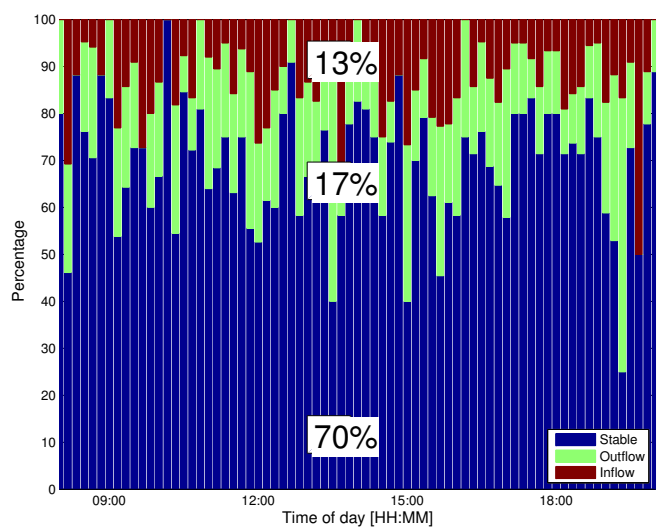

(h) Sector Nicky High.

Figure D.1: Sector occupancy count inflow \& outflow for multiple sectors. Look-ahead is 10 minutes. 


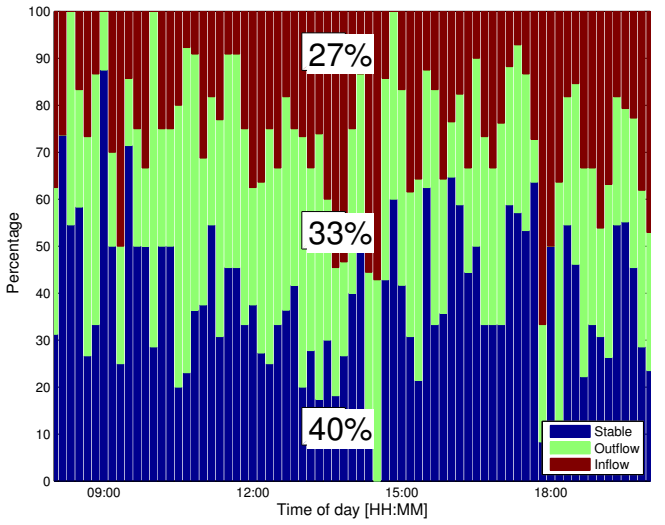

(a) Sector Jever Low.

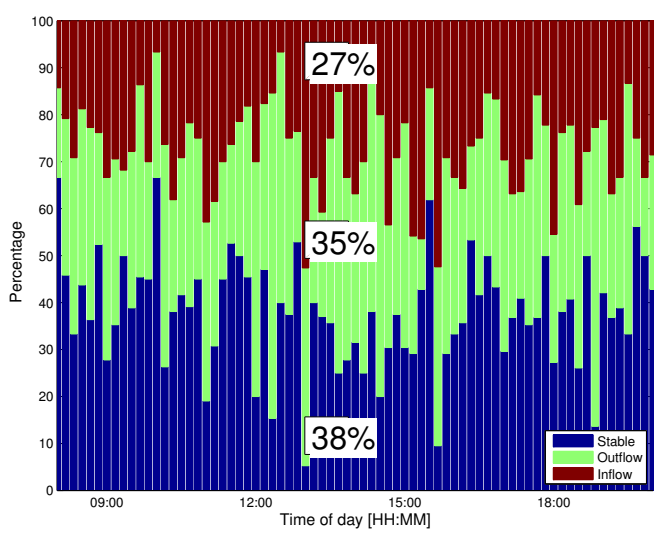

(c) Sector Koksy Low.

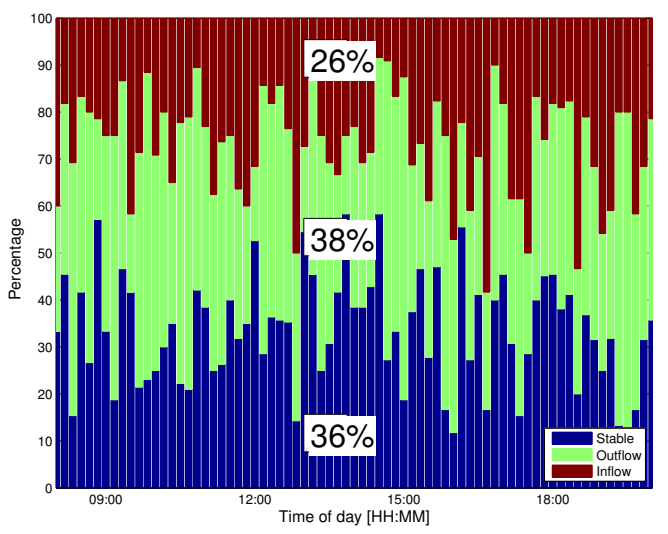

(e) Sector Koksy Low, 05/03/2014.

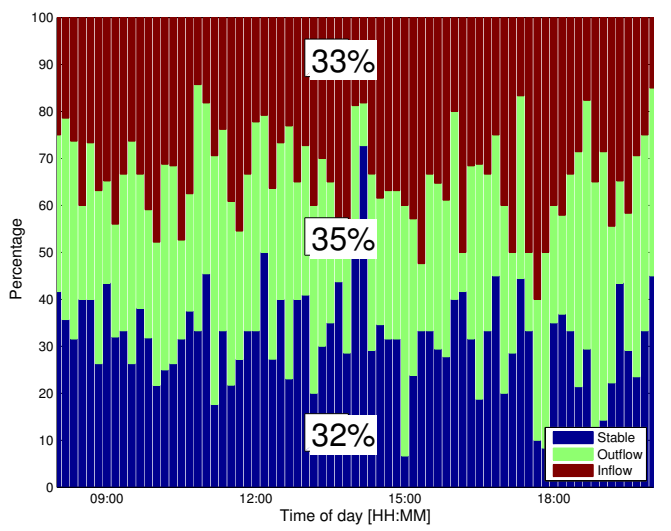

(g) Sector Lux Low.

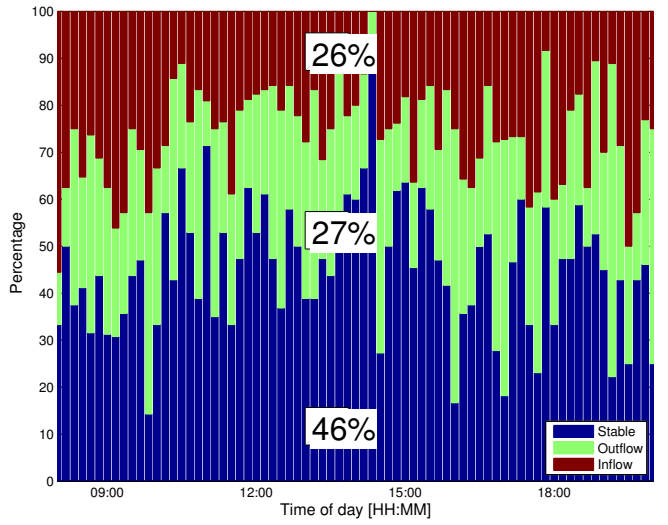

(b) Sector Koksy High.

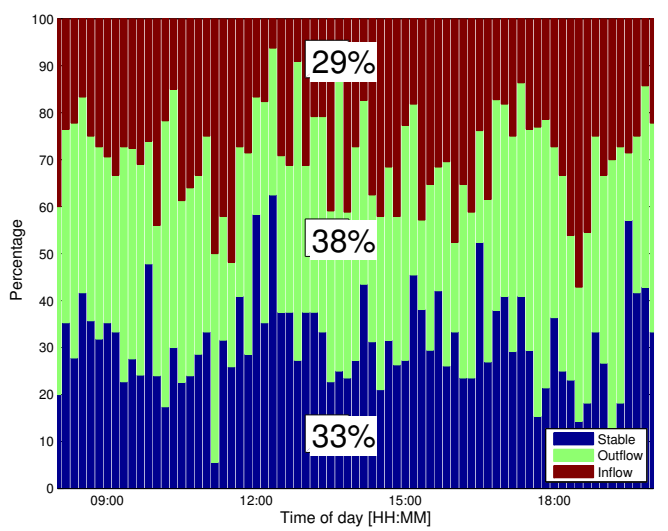

(d) Sector Koksy Low, 29/06/2013.

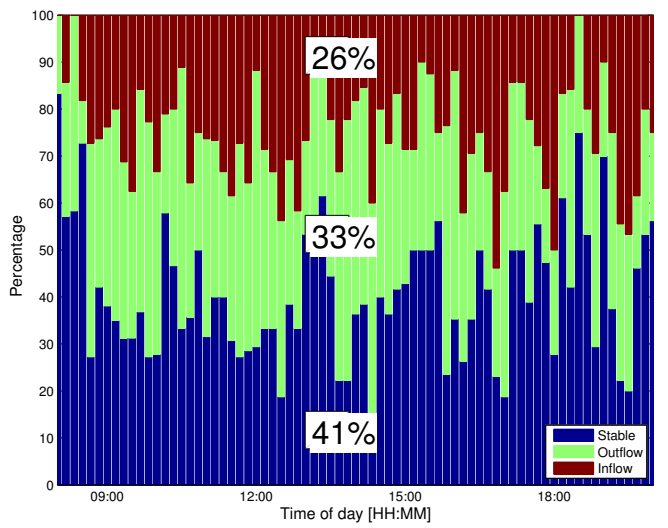

(f) Sector Bordeaux X4.

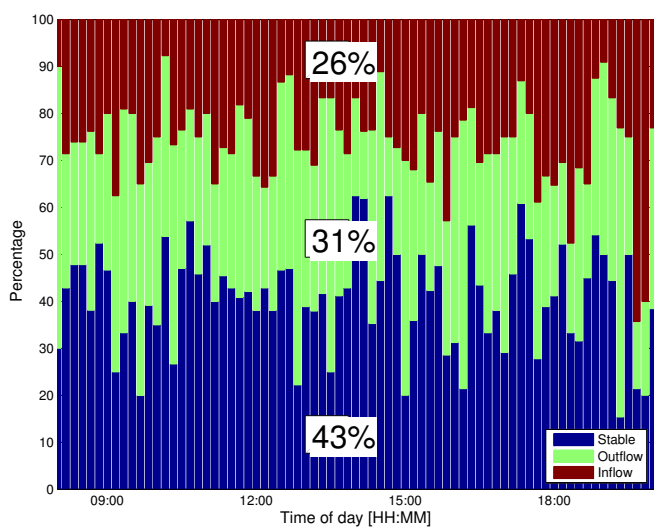

(h) Sector Nicky High.

Figure D.2: Sector occupancy count inflow \& outflow flights for multiple sectors. Lookahead is 60 minutes. 


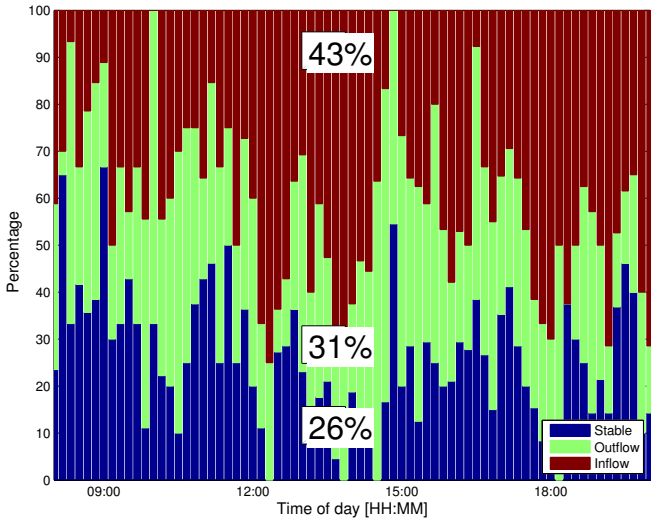

(a) Sector Jever Low.

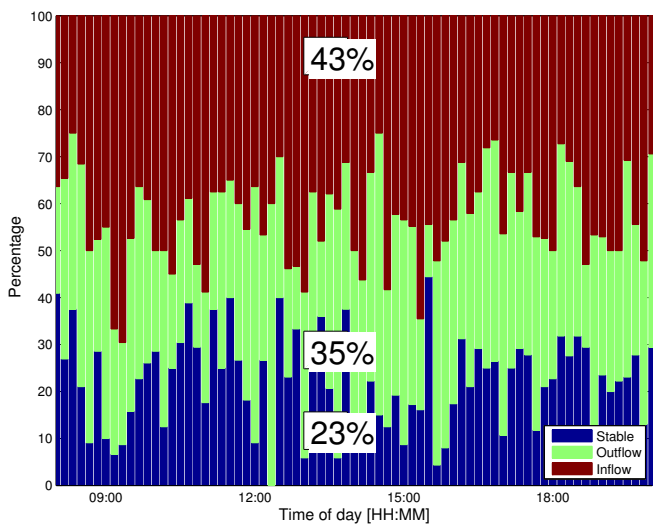

(c) Sector Koksy Low.

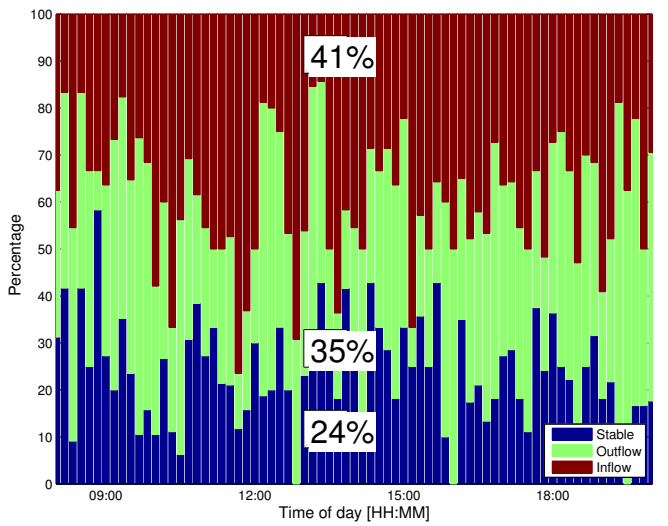

(e) Sector Koksy Low, 05/03/2014.

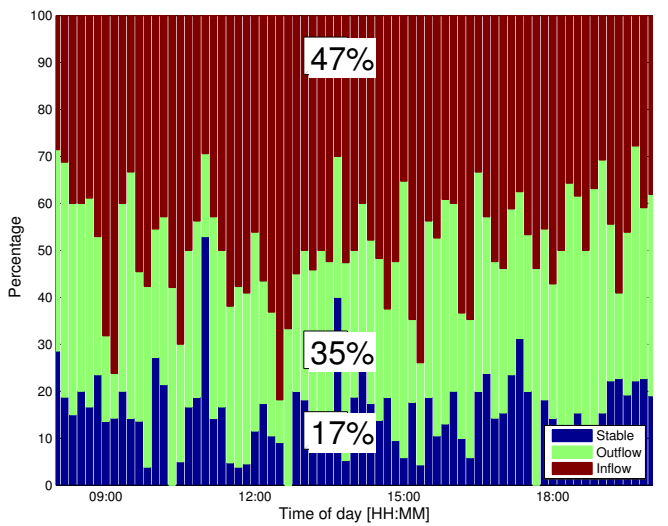

(g) Sector Lux Low.

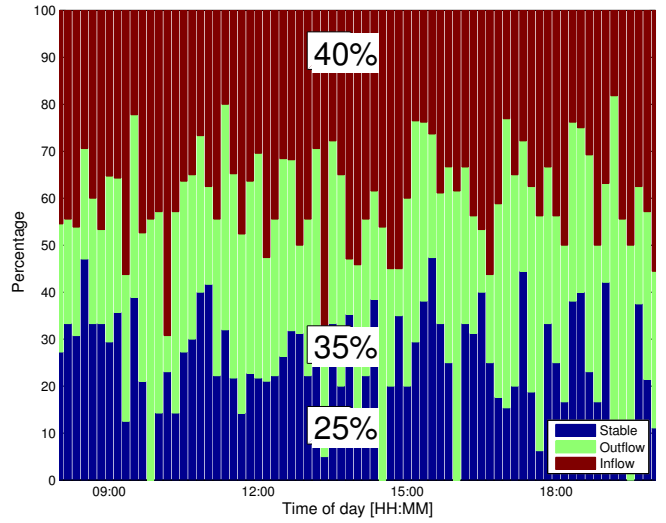

(b) Sector Koksy High.

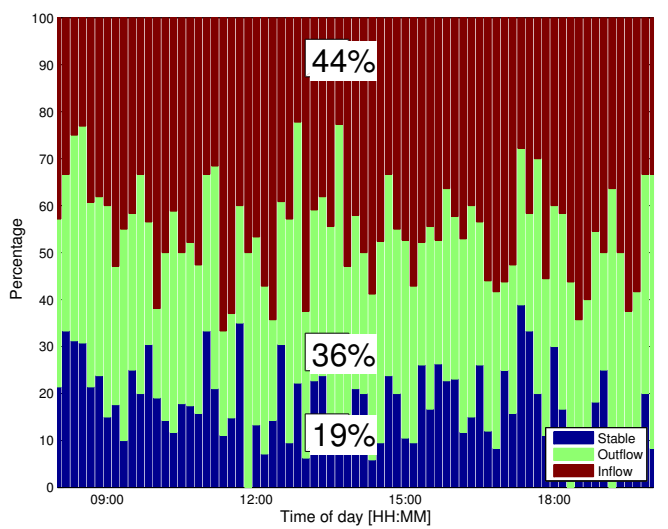

(d) Sector Koksy Low, 29/06/2013.

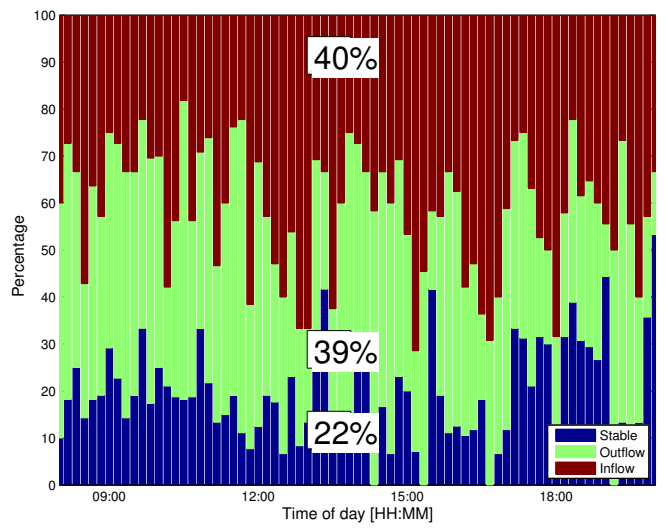

(f) Sector Bordeaux X4.

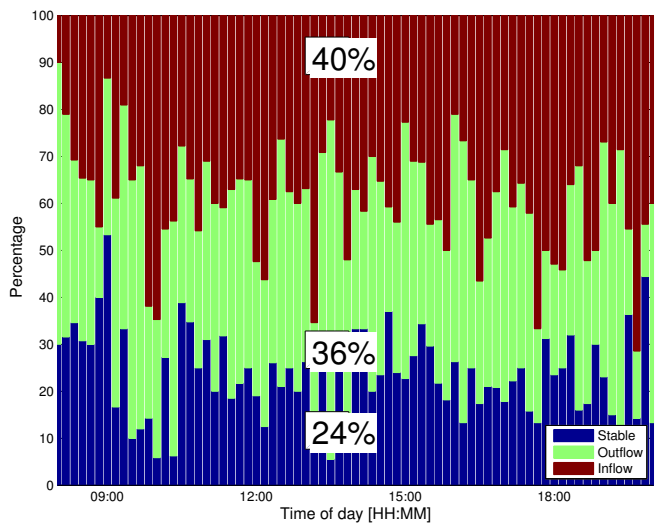

(h) Sector Nicky High.

Figure D.3: Sector occupancy count inflow \& outflow flights for multiple sectors. Lookahead time is 6 hours. 
Appendix E

Sector Entry Count Flight Status at Prediction 


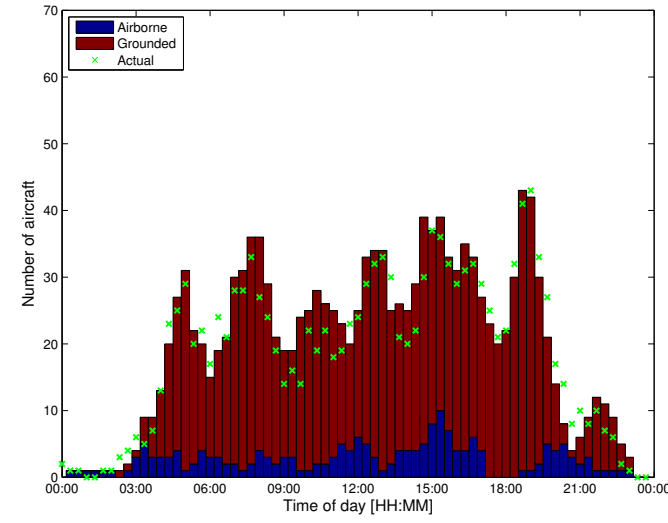

(a) Sector Jever Low.

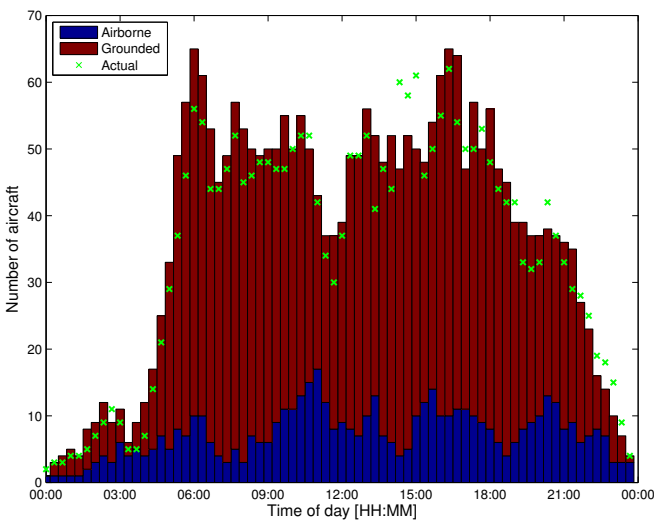

(c) Sector Koksy Low.

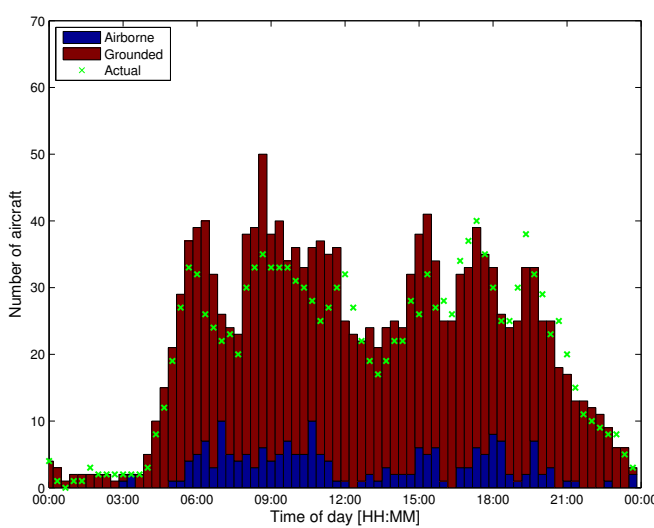

(e) Sector Bordeaux X4.

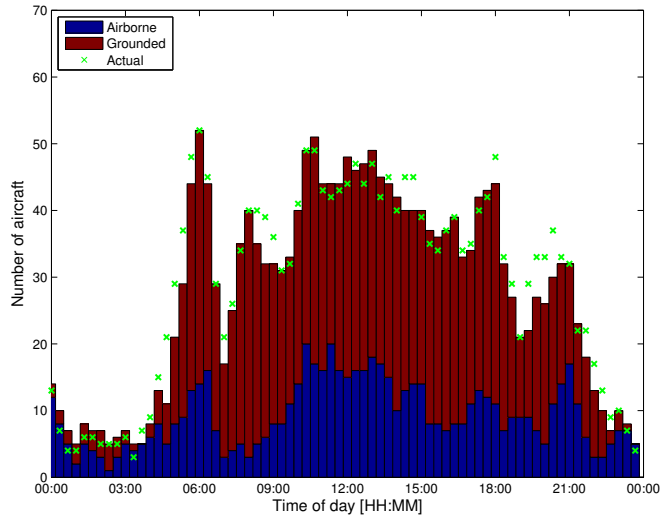

(b) Sector Koksy High.

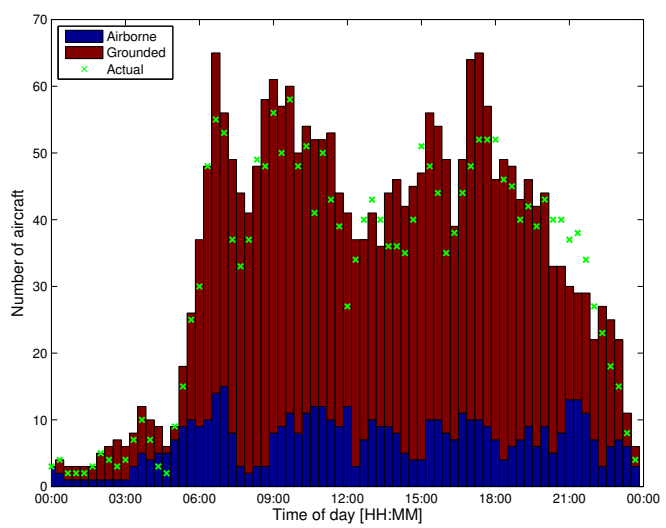

(d) Sector Koksy Low, 07/03/2014.

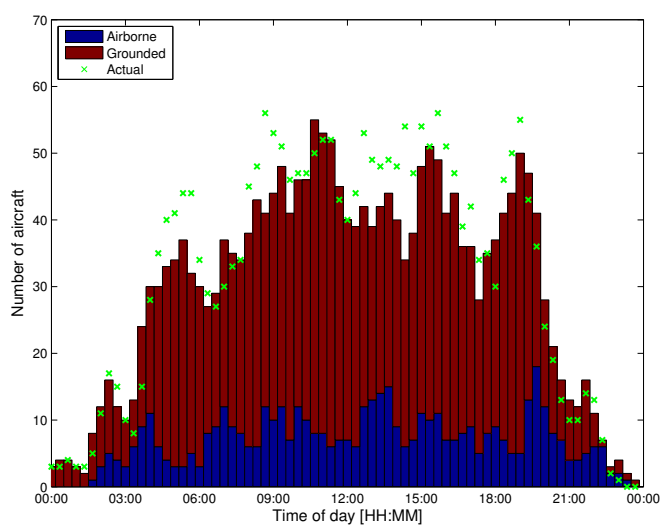

(f) Sector Lux Low.

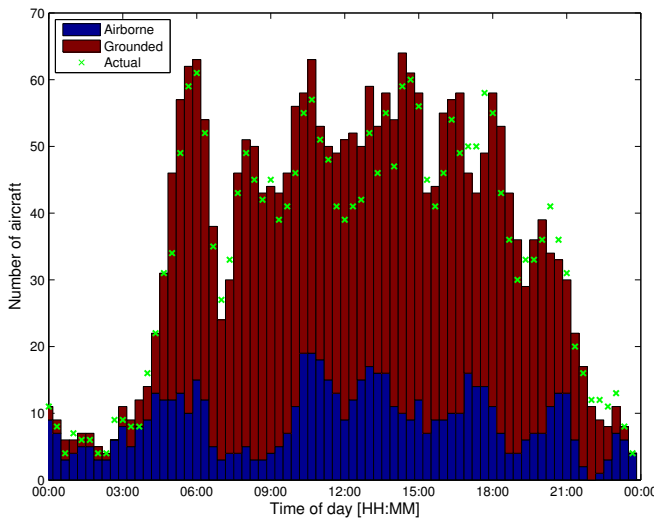

(g) Sector Nicky High.

Figure E.1: Sector entry count flight status at prediction for multiple sectors. Look-ahead is 60 minutes. 
Appendix F

\section{Sector Entry Count Inflow \& Outflow Flights}




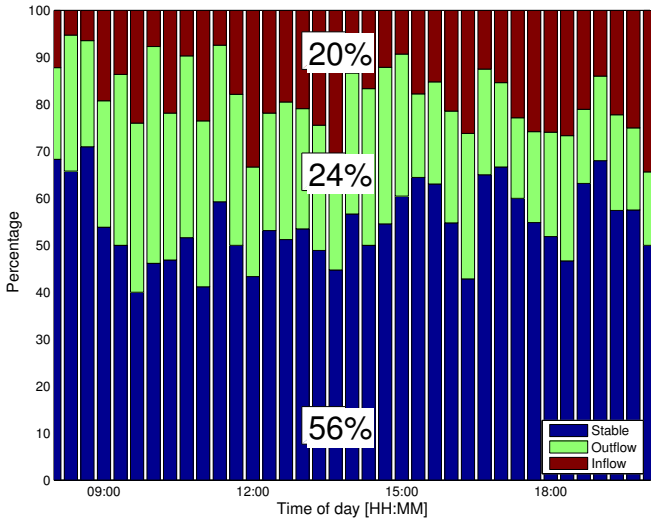

(a) Sector Jever Low.

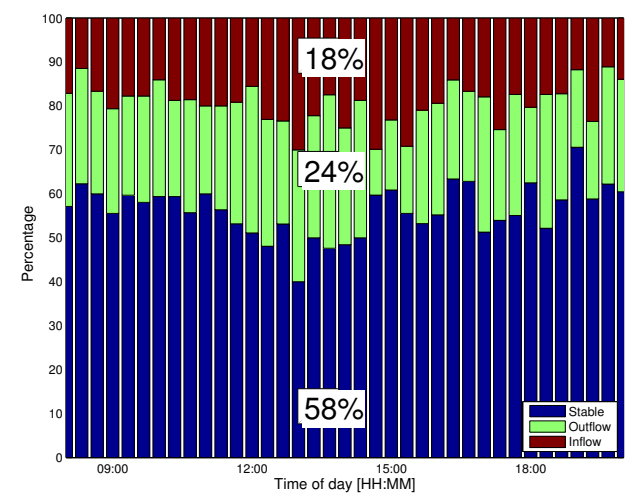

(c) Sector Koksy Low.

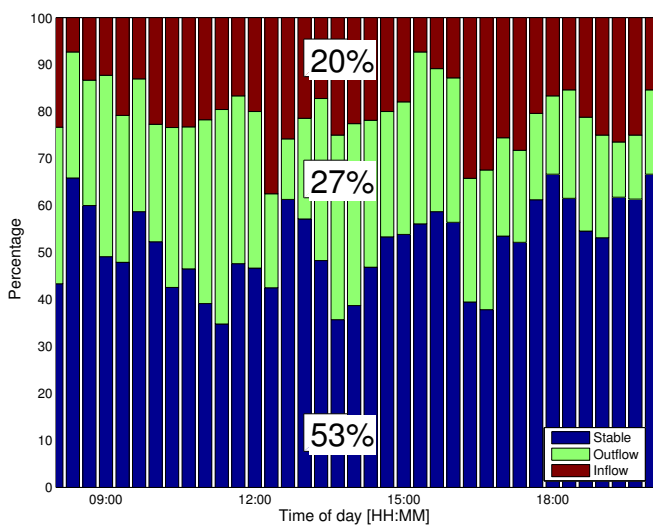

(e) Sector Bordeaux X4.

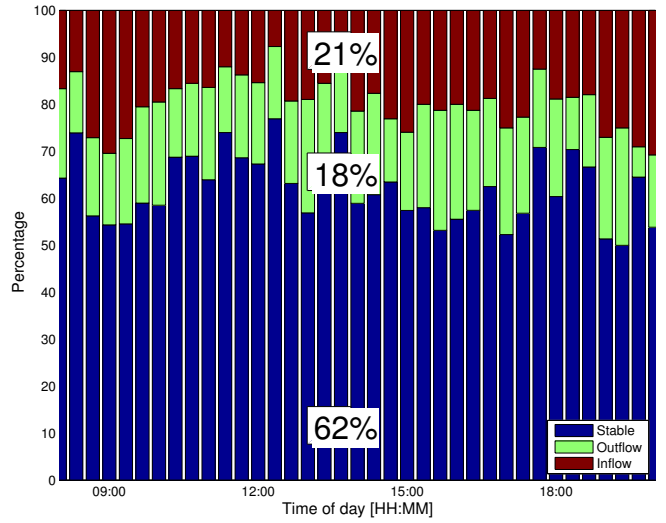

(b) Sector Koksy High.

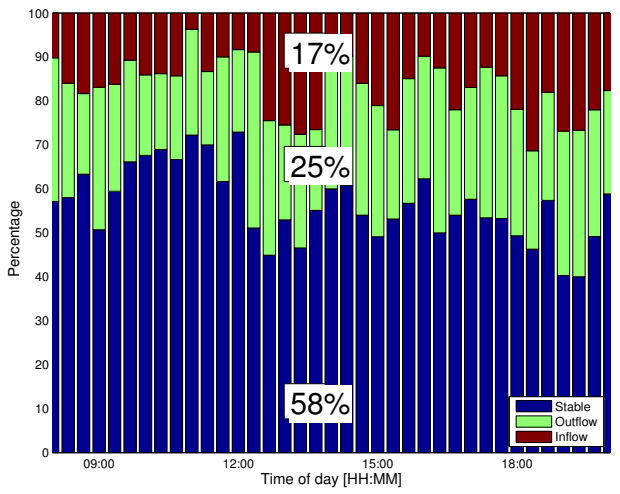

(d) Sector Koksy Low, 07/03/2014.

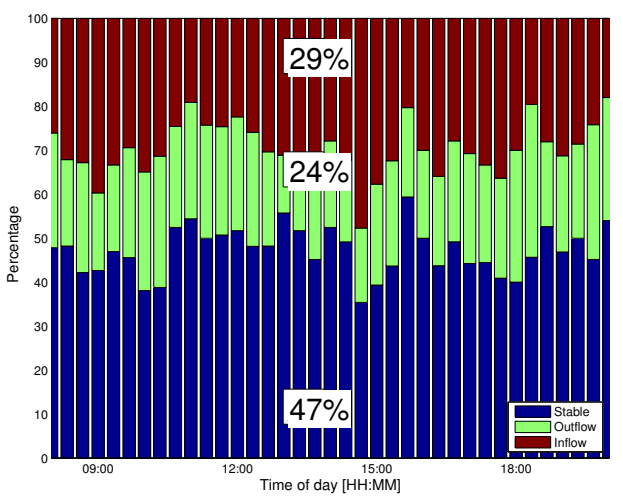

(f) Sector Lux Low.

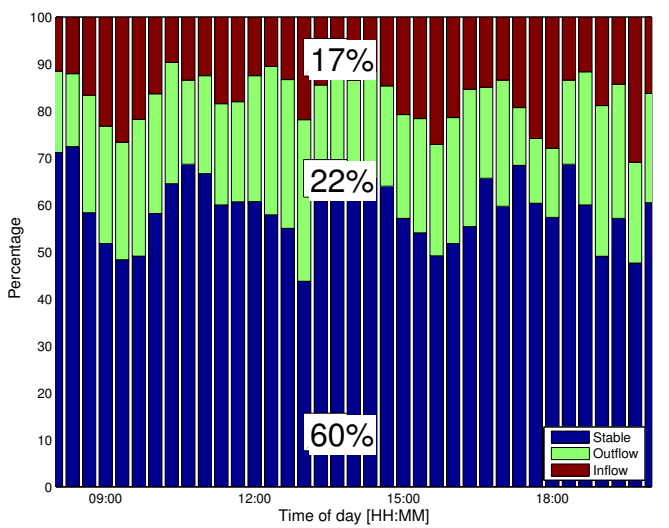

(g) Sector Nicky High.

Figure F.1: Sector entry count inflow \& outflow flights for multiple sectors. Look-ahead is 60 minutes. 